\title{
Modelos de regressão estáticos \\ e dinâmicos para taxas ou proporções; uma abordagem Bayesiana
}

\author{
Leandro Tavares Correia
}

TESE APRESENTADA

$\mathrm{AO}$

Instituto De Matemática e Estatística

DA

UniversidAdE DE SÃO PAUlo

PARA

OBTENÇÃO DO TÍTULO

$\mathrm{DE}$

DOUTOR EM CIÊNCIAS

\author{
Programa: Estatística \\ Orientador: Prof. Dr. Heleno Bolfarine \\ Coorientador: Prof ${ }^{\mathrm{a}}$. Dr ${ }^{\mathrm{a}}$. Cibele Queiroz da-Silva
}

Durante o desenvolvimento deste trabalho o autor recebeu auxílio financeiro da CAPES/CNPq

São Paulo, junho de 2015 


\section{Modelos de regressão estáticos \\ e dinâmicos para taxas ou proporções; \\ uma abordagem Bayesiana}

Esta é a versão original da tese elaborada pelo candidato Leandro Tavares Correia, tal como submetida à Comissão Julgadora. 


\section{Modelos de regressão estáticos e dinâmicos para taxas ou proporções; uma abordagem Bayesiana}

Esta versão da tese contém as correções e alterações sugeridas pela Comissão Julgadora durante a defesa da versão original do trabalho, realizada em 01/06/2015. Uma cópia da versão original está disponível no Instituto de Matemática e Estatística da Universidade de São Paulo.

Comissão Julgadora:

- Prof $^{\mathrm{a}}$. Dra ${ }^{\mathrm{a}}$. Cibele Queiroz da-Silva (coorientadora) - UnB

- Prof. Dr. Hedibert Lopes - INSPER

- Prof. Dr. Helio dos Santos Migon - UFRJ

- Prof. Dr. Josemar Rodrigues - ICMC-USP

- Prof. Dr. Jorge Luis Bazán Guzmán - ICMC-USP 


\section{Agradecimentos}

Agradeço primeiramente aos meus orientadores Heleno Bolfarine e Cibele Queiroz da-Silva pela paciência e tempo dedicado ao meu trabalho. Ao professor Hedibert Lopes que contribuiu em grande parcela no meu trabalho e sempre se colocou com disponibilidade para eventuais discussões e esclarecimento de dúvidas.

Agradeço, também, aos demais professores dos institutos IME-USP e Est-UnB pelo conhecimento compartilhado e por sempre se mostrarem solícitos para qualquer dúvida. Aos colegas de curso que também tiveram sua parcela de contribuição.

Por fim, à Capes e CNPQ pelo apoio financeiro. 


\section{Resumo}

\section{CORREIA, L. T. Modelos de regressão estáticos e dinâmicos}

para taxas ou proporções; uma abordagem Bayesiana. 2015. 186 f. Tese (Doutorado) - Instituto de Matemática e Estatística, Universidade de São Paulo, São Paulo, 2015.

Este trabalho apresenta um estudo de dados com resposta em intervalos limitados, mais especificamente no intervalo $[0,1]$, como no caso de taxas e proporções. Em diversos casos práticos esta estrutura de dados apresenta uma quantidade não negligenciável de valores extremos (0 e 1) e que modelos usuais não são adequados para sua análise. Para esta situação propomos, por meio de um enfoque Bayesiano, modelos de regressão beta inflacionado de zeros e uns (BIZU) e modelos de regressão Tobit duplamente censurado adaptados nesse intervalo. Técnicas de diagnóstico e qualidade do ajuste também são discutidas. Apresentamos a análise desta estrutura de dados no contexto de série de tempo por meio da abordagem Bayesiana de modelos dinâmicos. Estudos de comportamento e previsão de séries de tempo foram explorados utilizando técnicas de Monte Carlo sequencial, conhecidas como filtro de partículas. Particularidades e competitividade entre as duas classes de modelos também foram discutidas.

Palavras-chave: proporções, regressão, modelos dinâmicos, Distribuição beta, Modelo beta inflacionado, modelo Tobit, análise Bayesiana, filtro de partículas. 


\section{Abstract}

CORREIA, L. T. Regression of static and dynamic models for proportions or rates; a Bayesian approach. 2015. 186 f. Tese (Doutorado) - Instituto de Matemática e Estatística, Universidade de São Paulo, São Paulo, 2015.

This paper presents a study focused on observations in a limited interval, more specifically in $[0,1]$, such as rate and proportion data. In many practical cases this data structure has a considerable amount of extreme values ( 0 and 1$)$ and usual classical models are not suitable for this type of data set. We propose two class of regression models to deal with this context: beta inflated of zeros and ones $(B I Z U)$ models and Tobit doubly censored models adapted in this interval. Fit quality and diagnostic techniques are also discussed. Time series of proportions are also developed through Bayesian dynamic models. Forecasting and behavioral analysis were explored using sequential Monte Carlo techniques, known as particle filters. Particularities and competitiveness between the two classes of models were also discussed as well.

Keywords: proportions, regression, dynamic models, beta distribution, beta inflated model, Tobit model, Bayesian analysis, particle filters. 


\section{Sumário}

Lista de Figuras $\quad$ xi

$\begin{array}{lll}\text { Lista de Tabelas } & \text { xv }\end{array}$

1 Introdução $\quad \mathbf{1}$

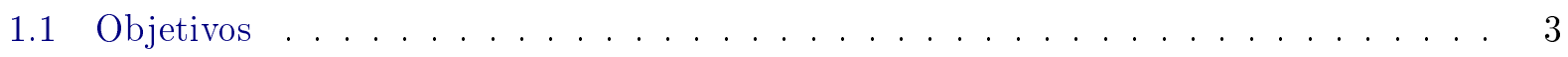

1.2 Organização do Trabalho . . . . . . . . . . . . . . . . . 3

2 Mistura de distribuições $\quad 5$

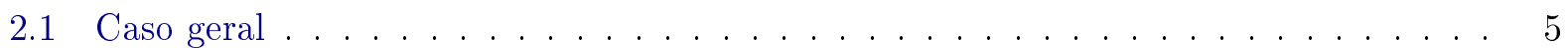

2.2 Distribuição Beta inflacionada de zeros e/ou uns . . . . . . . . . . . . . 6

2.3 Distribuição Tobit normal duplamente censurada e inflacionada de zeros e/ou uns . . 10

2.4 Distribuição Tobit normal-potência duplamente censurada e inflacionada de zeros

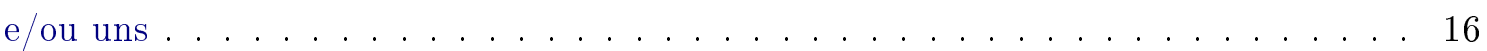

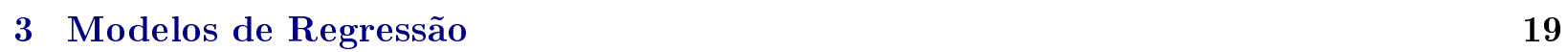

3.1 Modelos de regressão beta inflacionado . . . . . . . . . . . . . . . . . . . 19

3.2 Modelos de regressão Tobit normal duplamente censurado e inflacionado . . . . . . . 22

3.3 Modelos de regressão Tobit normal-potência duplamente censurado e inflacionado . . 24

3.4 Inferência Bayesiana . . . . . . . . . . . . . . . . . . . . . 26

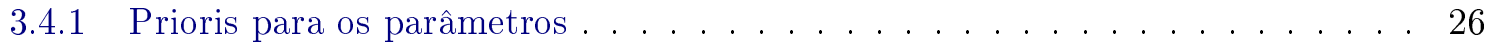

3.4.2 Obtenção das distribuições a posteriori para os parâmetros . . . . . . . . 28

3.5 Medidas de diagnóstico . . . . . . . . . . . . . . . . . . . . . . . 29

3.5.1 Critérios de escolha do modelo . . . . . . . . . . . . . . . . . 29

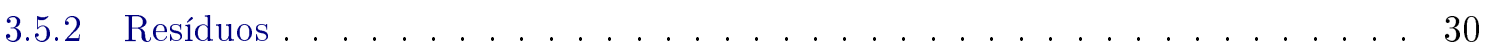

3.5.3 Pontos influentes . . . . . . . . . . . . . . . . . . . 31

3.6 Estudo de simulação . . . . . . . . . . . . . . . . . . . . 33

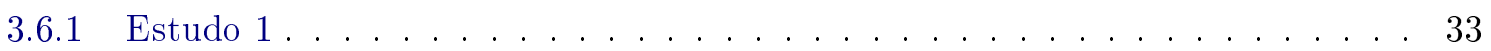

3.6 .2 Estudo $2 \ldots \ldots \ldots \ldots \ldots \ldots \ldots$

3.6 .3 Conclusão . . . . . . . . . . . . . . . . . . . . . . . 43

3.7 Aplicações . . . . . . . . . . . . . . . . . . . . . . . . 43

3.7.1 Proporções de empregados do sexo feminino no setor de indústria . . . . . . . 43

3.7 .2 Proporções de canais . . . . . . . . . . . . . . . . . . . . 50 
4.1 Abordagem Bayesiana em Modelos Dinâmicos . . . . . . . . . . . . . . . . 55

4.2 Modelo dinâmico beta inflacionado . . . . . . . . . . . . . . . . . 56

4.2 .1 Análise Bayesiana . . . . . . . . . . . . . . . . 57

4.2 .2 Primeiro resultado com simulações via MCMC $\ldots \ldots \ldots$. . . . . . . . . . 59

4.3 Modelo dinâmico Tobit-normal . . . . . . . . . . . . . . . . . . . 63

4.3.1 Modelo dinâmico Tobit normal duplamente censurado . . . . . . . . . . 66

5 Filtro de Partículas $\quad 69$

5.1 Caso geral . . . . . . . . . . . . . . . . . . . . . . 69

5.1 .1 Filtro no contexto da modelagem Bayesiana . . . . . . . . . . . . . . 71

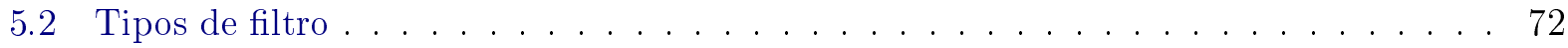

5.2 .1 Filtro de particulas bootstrap . . . . . . . . . . . . . 72

5.2 .2 Filtro de partículas auxiliar . . . . . . . . . . . . . . 73

5.3 Estimação dos parâmetros . . . . . . . . . . . . . . . . . . . . 76

5.3 .1 Filtro Liu e West . . . . . . . . . . . . . . . . . . 76

$5.3 .2 \quad$ Filtro Storvik . . . . . . . . . . . . . . . . . . . 77

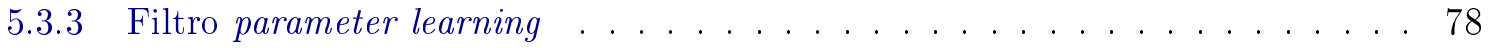

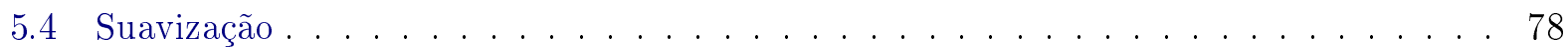

6 Simulação e aplicação $\quad 81$

6.1 Estudo de simulação para o modelo dinâmico BIZU . . . . . . . . . . . . . . . . . . 81

6.1 .1 Modelo de passeio aleatório . . . . . . . . . . . . . . . 82

6.1 .2 Modelo com nível, tendência e sazonalidade . . . . . . . . . . . . . . 96

6.1.3 Modelo com covariáveis . . . . . . . . . . . . . . . . . . . . . . . . . . . 104

6.2 Estudo de simulação para o modelo dinâmico Tobit ～. . . . . . . . . . . . . . . 112

6.2.1 Modelo com tendência e sazonalidade . . . . . . . . . . . . . . . . . . . . 112

6.2 .2 Modelo com covariáveis . . . . . . . . . . . . . . . . . . . . . . . . 119

6.3 Aplicação . . . . . . . . . . . . . . . . . . . . . 126

7 Conclusões $\quad 143$

7.1 Considerações Finais . . . . . . . . . . . . . . . . . . . . . . . . . . 143

7.2 Sugestões para Pesquisas Futuras . . . . . . . . . . . . . . . . . . . 143

$\begin{array}{ll}\text { A Métricas } & 145\end{array}$

B Intervenção externa $\quad 147$

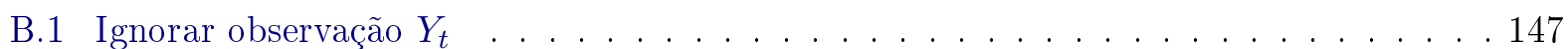

B.2 Ruído de evolução adicional . . . . . . . . . . . . . . . . . . . 147

B.3 Intervenção subjetiva arbitrária . . . . . . . . . . . . . . . . . . . . . 147

B.4 Inclusão de efeito de intervenção ～. . . . . . . . . . . . . . . . . . . . 148

C Modelo linear dinâmico generalizado $\quad 149$ 
$\begin{array}{ll}\text { D Principais programações em R } & 151\end{array}$

D.1 Simulação dos dados BIZU dinâmico . . . . . . . . . . . . . . . . . 151

D.2 Simulação dos dados Tobit dinâmico . . . . . . . . . . . . . . . 153

D.3 Filtro PL para o modelo dinâmico BIZU . . . . . . . . . . . . . . . . . 155

D.4 Filtro PL para o modelo dinâmico Tobit . . . . . . . . . . . . . . . . 158

Referências Bibliográficas 


\section{Lista de Figuras}

2.1 Gráfico das densidades beta para diferentes valores de $(\mu, \phi) \ldots \ldots \ldots \ldots$

2.2 Gráfico das funções de distribuição acumulada das variáveis $B I_{0}$ (esquerda) e $B I Z U$ (direita), considerando os parâmetros $(\alpha=0.24, \mu=0.6, \phi=5)$ e $(\alpha=0.39, \gamma=$

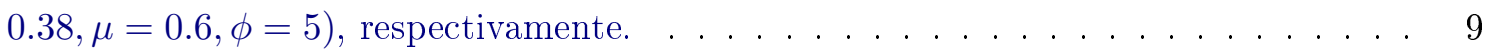

2.3 Gráfico das funções de verossimilhança das variáveis $B I_{0}$ (esquerda) e $B I Z U$ (direita), considerando os parâmetros $(\alpha=0.24, \mu=0.6, \phi=5)$ e $(\alpha=0.39, \gamma=$

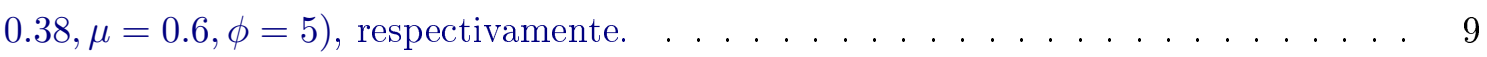

2.4 Gráfico das densidades Tobit normal inflacionada para diferentes valores de $(\mu, \sigma)$. $\quad 14$

2.5 Gráfico das funções de distribuição acumulada das variáveis tobit normal inflacionada de zeros (esquerda) e tobit normal inflacionada de zeros e uns (direita), considerando os parâmetros $(\delta=0.24, \mu=0.6, \sigma=0.3)$ e $\left(\delta_{0}=0.24, \delta_{1}=0.15, \mu=0.6, \sigma=0.3\right)$,

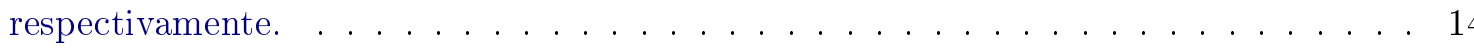

2.6 Gráfico das funções de verossimilhança das variáveis Tobit normal inflacionada de zeros (esquerda) e tobit normal inflacionada de zeros e uns (direita), considerando os parâmetros $(\delta=0.24, \mu=0.6, \sigma=0.3)$ e $\left(\delta_{0}=0.24, \delta_{1}=0.15, \mu=0.6, \sigma=0.3\right)$,

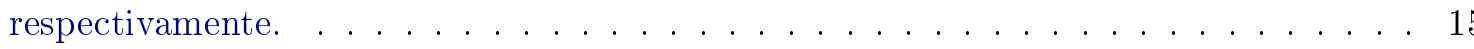

2.7 Gráfico das densidades Tobit normal-potência inflacionada para diferentes valores de

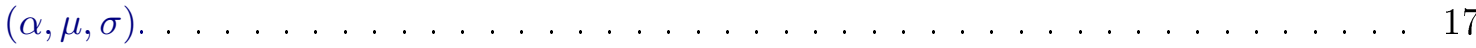

2.8 Gráfico das funções de distribuição acumulada das variáveis Tobit normal-potência inflacionada de zeros (esquerda) e Tobit normal-potência inflacionada de zeros e uns (direita), considerando os parâmetros $(\delta=0.24, \alpha=0.5, \mu=0.6, \sigma=0.3)$ e

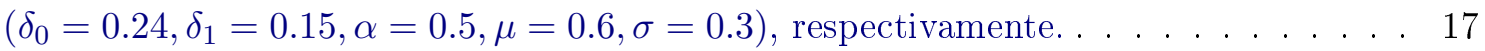

2.9 Gráfico das funções de verossimilhança das variáveis Tobit normal-potência inflacionada de zeros (esquerda) e Tobit normal-potência inflacionada de zeros e uns (direita), considerando os parâmetros $(\delta=0.24, \mu=0.6, \sigma=0.3)$ e $\left(\delta_{0}=0.24, \delta_{1}=\right.$

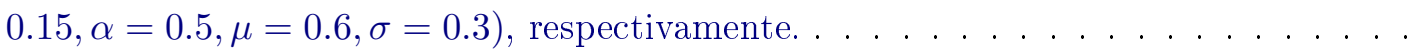

3.1 Gráfico dos resíduos Bayesianos padronizados pelas observações para os modelos BIZU (esquerda) e Tobit inflacionado (direita).

3.2 Gráfico dos resíduos Bayesianos condicionais padronizados pelas observações para os modelos BIZU (esquerda) e Tobit inflacionado (direita) . . . . . . . . . . . . . 36

3.3 Gráfico da divergência de K-L para o modelo BIZU (esquerda) e Tobit inflacionado

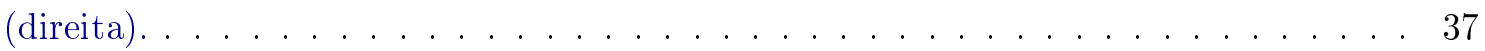


3.4 Gráfico dos resíduos Bayesianos padronizados pelas observações para os modelos BIZU (esquerda) e Tobit inflacionado (direita) . . . . . . . . . . . . . . . . 41

3.5 Gráfico dos resíduos Bayesianos condicionais padronizados pelas observações para os modelos BIZU (esquerda) e Tobit inflacionado (direita) . . . . . . . . . . . . . . . 41

3.6 Gráfico da divergência de K-L para o modelo BIZU (esquerda) e Tobit inflacionado

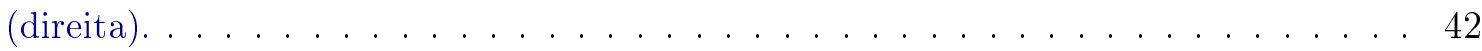

3.7 Gráfico dos resíduos Bayesianos padronizados pelas observações para os modelos BIZU (esquerda superior), Tobit normal inflacionado (direita superior) e Tobit normal potência inflacionado (inferior). . . . . . . . . . . . . .

3.8 Gráfico dos resíduos Bayesianos condicionais padronizados pelas observações para os modelos BIZU (esquerda superior), Tobit normal inflacionado (direita superior) e Tobit normal potência inflacionado (inferior). . . . . . . . . . . . . . . 47

3.9 Gráfico da divergência de K-L para os modelos BIZU (esquerda superior), Tobit normal inflacionado (direita superior) e Tobit normal potência inflacionado (inferior). 48

3.10 Gráfico da distância- $\chi^{2}$ para os modelos BIZU (esquerda superior), Tobit normal inflacionado (direita superior) e Tobit normal potência inflacionado (inferior). . . . . 49

3.11 Gráfico dos resíduos Bayesianos padronizados pelas observações para os modelos BIZ (esquerda) e Tobit inflacionado (direita) . . . . . . . . . . . . . . . 52

3.12 Gráfico dos resíduos Bayesianos padronizados pelas observações para o modelo tobit normal-potência inflacionado. . . . . . . . . . . . . . . . 52

3.13 Gráfico da distância- $\chi^{2}$ para o modelo BIZ (esquerda) e tobit inflacionado (direita). . 53

3.14 Gráfico da distância- $\chi^{2}$ para o modelo tobit normal-potência. . . . . . . . . . . . 53

4.1 Estrutura do modelo linear dinâmico . . . . . . . . . . . . . . . . 56

4.2 Gráfico de ajuste dos efeitos de nível, tendência e sazonalidade para a média com

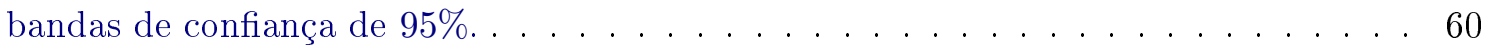

4.3 Gráfico de ajuste dos efeitos de nível e tendência para a precisão com bandas de

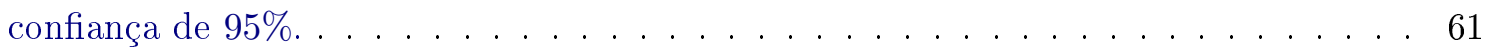

4.4 Gráfico de ajuste dos preditores lineares do modelo com bandas de confiança de $95 \%$. 61

4.5 Gráfico das distribuições a posteriori dos parâmetros estáticos $\alpha$ e $\gamma$. . . . . . . . 62

6.1 Gráficos dos dados simulados do modelo BIZU com passeio aleatório (linha contínua) e seu parâmetro $\mu_{t}$ (linha pontilhada) . . . . . . . . . . . . 83

6.2 Gráficos dos preditores lineares de $\mu_{t}$ e $\phi_{t}$ do modelo BIZU estimados via filtro LW. 83

6.3 Gráfico da estimativa das componentes da variância $W$ do modelo $B I Z U$ via filtro

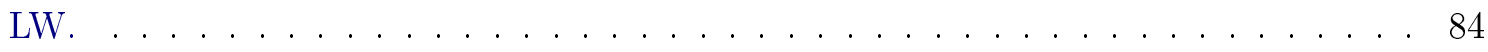

6.4 Gráfico da estimativa das probabilidades $\alpha$ e $\gamma$ do modelo BIZU via filtro LW. . . 85

6.5 Gráficos dos preditores lineares de $\mu_{t}$ e $\phi_{t}$ do modelo BIZU estimados via filtro PL. $\quad 86$

6.6 Gráfico da estimativa das componentes da variância $W$ do modelo $B I Z U$ via filtro

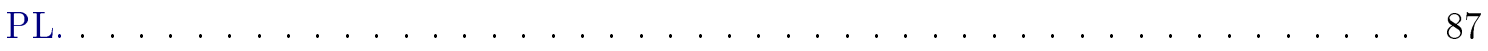

6.7 Gráfico da estimativa das probabilidades $\alpha$ e $\gamma$ do modelo $B I Z U$ via filtro PL. . . . 88

6.8 Gráficos dos dados simulados do modelo BIZU com passeio aleatório (linha contínua)

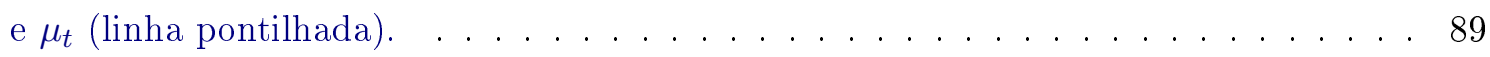

6.9 Gráficos dos preditores linearesde $\mu_{t}$ e $\phi_{t}$ do modelo BIZU estimados via filtro LW. 90 
6.10 Gráfico da estimativa das componentes da variância $W$ do modelo $B I Z U$ via filtro

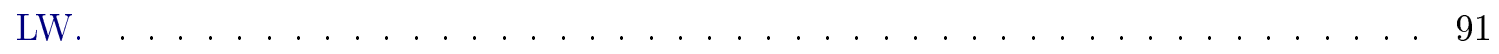

6.11 Gráfico da estimativa das probabilidades $\alpha$ e $\gamma$ do modelo BIZU via filtro LW. . . . 92

6.12 Gráficos dos preditores lineares de $\mu_{t}$ e $\phi_{t}$ do modelo $B I Z U$ estimados via filtro PL. $\quad 93$

6.13 Gráfico da estimativa das componentes da variância $W$ do modelo $B I Z U$ via filtro

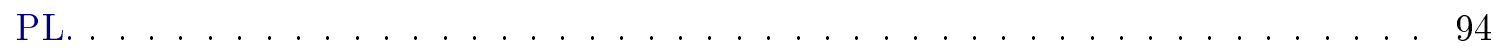

6.14 Gráfico da estimativa das probabilidades $\alpha$ e $\gamma$ do modelo BIZU via filtro PL. . . . 95

6.15 Gráfico da série simulada do modelo dinâmico BIZU com nível, tendência e sazonalidade, com $y_{t}$ (linha continua) e $\mu_{t}$ (linha pontilhada) . . . . . . . . . . . 97

6.16 Gráfico dos preditores lineares de $\mu_{t}$ e $\phi_{t}$ do modelo dinâmico BIZU. . . . . . . . . 97

6.17 Gráfico dos estados $\mu_{t}$ e $\phi_{t}$ do modelo dinâmico BIZU. . . . . . . . . . . . . . . 98

6.18 Gráfico do desvio padrão estimado da componente contínua do modelo dinâmico BIZU. 99

6.19 Gráfico das principais componentes da matriz de variância $W$ estimados no modelo

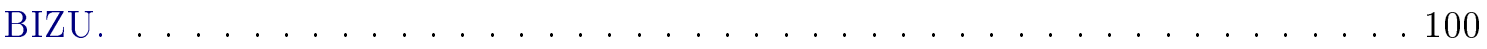

6.20 Gráfico da estimativa das probabilidades $\alpha$ e $\gamma$ do modelo BIZU. . . . . . . . . . . 101

6.21 Gráfico dos preditores lineares $\eta_{t}$ suavizados em comparação aos estimados via o processo de filtragem. . . . . . . . . . . . . . . . . . . . . 102

6.22 Gráfico da distribuição preditiva $p\left(y_{t} \mid D_{t-1}\right)$ da componente contínua do modelo dinâmico BIZU. . . . . . . . . . . . . . . . . . . . . . . . . . . 102

6.23 Gráfico da distribuição preditiva $p\left(y_{t} \mid D_{t-1}\right)$ da componente contínua do modelo dinâmico BIZU com previsão a 10 passos a frente. . . . . . . . . . . . . . . . . . . . 103

6.24 Gráfico da série simulada do modelo dinâmico BIZU com nível, tendência e sazonalidade e covariáveis, com $y_{t}$ (linha contínua) e $\mu_{t}$ (linha pontilhada). . . . . . . . . . 105

6.25 Gráfico dos preditores lineares de $\mu_{t}$ e $\phi_{t}$ do modelo dinâmico BIZU. . . . . . . . . 106

6.26 Gráfico dos estados $\mu_{t}$ e $\phi_{t}$ do modelo dinâmico BIZU. . . . . . . . . . . . . . . . 107

6.27 Gráfico do desvio padrão estimado da componente contínua do modelo dinâmico BIZU.107

6.28 Gráfico das principais componentes da matriz de variância $W$ estimados no modelo

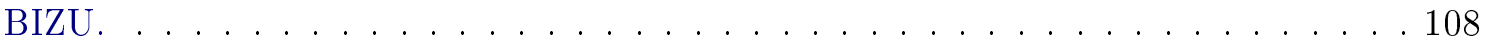

6.29 Gráfico da estimativa das parâmetros das covariáveis do modelo BIZU. . . . . . . . . 109

6.30 Gráfico da estimativa das probabilidades $\alpha$ e $\gamma$ do modelo BIZU. . . . . . . . . . . 110

6.31 Gráfico da distribuição preditiva $p\left(y_{t} \mid D_{t-1}\right)$ da componente contínua do modelo dinâmico BIZU. . . . . . . . . . . . . . . . . . . . . 110

6.32 Gráfico da distribuição preditiva $p\left(y_{t} \mid D_{t-1}\right)$ da componente contínua do modelo dinâmico BIZU com previsão a 10 passos a frente. . . . . . . . . . . . . . . . 111

6.33 Gráfico da série simulada do modelo dinâmico Tobit com nível, tendência e sazonalidade, com $y_{t}$ (linha contínua) e $\mu_{t}$ (linha pontilhada) . . . . . . . . . . . . 113

6.34 Gráfico dos preditores lineares de $\mu_{t}$ e $\sigma_{t}$ do modelo dinâmico Tobit. . . . . . . . . . 114

6.35 Gráfico dos estados $\mu_{t}$ e $\sigma_{t}$ do modelo dinâmico Tobit. . . . . . . . . . . . . . 115

6.36 Gráfico das principais componentes da matriz de variância $W$ estimados no modelo

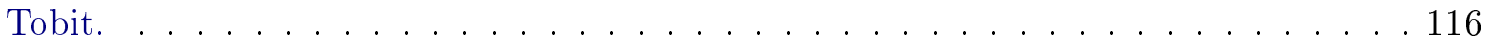

6.37 Gráfico dos preditores lineares suavizados em comparação ao estimados no processo

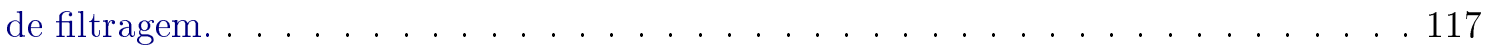

6.38 Gráfico da distribuição preditiva $p\left(y_{t} \mid D_{t-1}\right)$ do modelo dinâmico Tobit. . . . . . . . 117 
6.39 Gráfico da distribuição preditiva $p\left(y_{t} \mid D_{t-1}\right)$ do modelo dinâmico Tobit com previsão a 10 passos a frente. . . . . . . . . . . . . . . . . 118

6.40 Gráfico da série simulada do modelo dinâmico Tobit com nível, tendência e sazonalidade e covariáveis, com $y_{t}$ (linha contínua) e $\mu_{t}$ (linha pontilhada). . . . . . . . . 120

6.41 Gráfico dos preditores lineares de $\mu_{t}$ e $\sigma_{t}$ do modelo dinâmico Tobit. . . . . . . . . 120

6.42 Gráfico dos estados $\mu_{t}$ e $\sigma_{t}$ do modelo dinâmico Tobit. . . . . . . . . . . . . . . 121

6.43 Gráfico das principais componentes da matriz de variância $W$ estimados no modelo

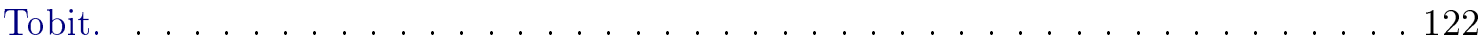

6.44 Gráfico da estimativa das parâmetros das covariáveis do modelo Tobit. . . . . . . . . 123

6.45 Gráfico dos preditores lineares suavizados em comparação ao estimados no processo de filtragem. . . . . . . . . . . . . . . . . . . . 124

6.46 Gráfico da distribuição preditiva $p\left(y_{t} \mid D_{t-1}\right)$ do modelo dinâmico Tobit. . . . . . . . . 124

6.47 Gráfico da distribuição preditiva $p\left(y_{t} \mid D_{t-1}\right)$ do modelo dinâmico Tobit com previsão a 10 passos a frente. . . . . . . . . . . . . . . . . . . 125

6.48 Ilustração de como funciona o sistema de abastecimento do sistema da Cantareira. . 127

6.49 Série de dados diária do nível do sistema da Cantareira do início de 2013 a março de

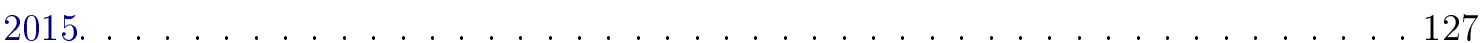

6.50 Gráfico da relação quadrática entre o sistema da cantareira com os demais sistemas a partir do ano de $2013 \ldots \ldots$. . . . . . . . . . . . . . . . . 128

6.51 Gráfico da relação quadrática entre o sistema da cantareira com os demais sistemas

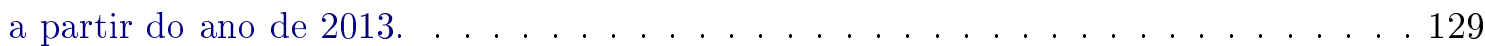

6.52 Gráfico da relação quadrática entre o sistema da cantareira com os demais sistemas a partir do ano de $2013 . \ldots$. . . . . . . . . . . . . . . . . . . 129

6.53 Gráfico da estimativa das componentes da variância $W$ do modelo dinâmico tobit. . 133

6.54 Gráfico da estimativa dos parâmetros $\beta$ do modelo dinâmico tobit. . . . . . . . . . . 134

6.55 Gráfico dos preditores lineares $\eta$ estimados pelo modelo dinâmico tobit. . . . . . . . 135

6.56 Gráfico dos estados $\mu_{t}$ e $\sigma_{t}$ do modelo dinâmico tobit. . . . . . . . . . . . . . 136

6.57 Grafico da distribuição preditiva $p\left(y_{t} \mid D_{t-1}\right)$ do modelo dinâmico tobit. . . . . . . . 136

6.58 Grafico da distribuição preditiva $p\left(y_{t} \mid D_{t-1}\right)$ com predição de 30 passos a frente do modelo dinâmico tobit. . . . . . . . . . . . . . . . . . . 137

6.59 Gráfico da estimativa das componentes da variância $W$ do modelo dinâmico BIZU. . 138

6.60 Gráfico da estimativa da componente discreta $\alpha$ do modelo dinâmico BIZU. . . . . . 139

6.61 Gráfico dos preditores lineares $\eta$ estimados pelo modelo dinâmico BIZU. . . . . . . . 139

6.62 Gráfico dos estados $\mu_{t}$ e $\phi_{t}$ do modelo dinâmico BIZU. . . . . . . . . . . . . . . . 140

6.63 Gráfico do desvio padrão da componente contínua do modelo dinâmico BIZU. . . . . 140

6.64 Gráfico da distribuição preditiva $p\left(y_{t} \mid D_{t-1}\right)$ da componente contínua do modelo dinâmico BIZU. . . . . . . . . . . . . . . . . . . . . . . . . . . . 141

6.65 Gráfico da distribuição preditiva $p\left(y_{t} \mid D_{t-1}\right)$ com predição de 30 passos a frente da componente contínua do modelo dinâmico BIZU. . . . . . . . . . . . . . . . . . . . . 141

6.66 Gráfico da distribuição preditiva $p\left(y_{t} \mid D_{t-1}\right)$ do modelo dinâmico beta. . . . . . . . 141

6.67 Gráfico da distribuição preditiva $p\left(y_{t} \mid D_{t-1}\right)$ com predição de 30 passos a frente do modelo dinâmico beta. . . . . . . . . . . . . . . . . . . . . . 142 


\section{Lista de Tabelas}

3.1 Comparação entre os modelos via DIC, $\widehat{E A I C}, \widehat{E B I C}$ e LMPL. . . . . . . . . . . . . 34

3.2 Estatísticas das distribuições marginais a posteriori dos parâmetros para o modelo

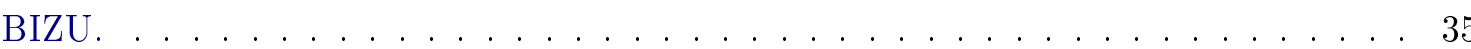

3.3 Estatísticas das distribuições marginais a posteriori dos parâmetros para o modelo

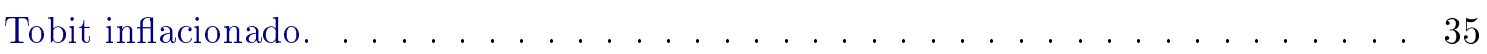

3.4 Comparação entre os modelos via DIC, $\widehat{E A I C}, \widehat{E B I C}$ e LMPL. . . . . . . . . . . . . 39

3.5 Estatísticas das distribuições marginais a posteriori dos parâmetros para o modelo

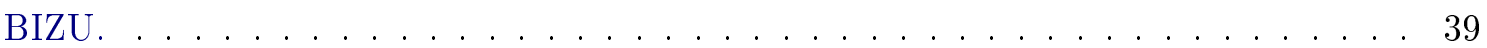

3.6 Estatísticas das distribuições marginais a posteriori dos parâmetros para o modelo Tobit inflacionado. . . . . . . . . . . . . . . . . . . . . 39

3.7 Comparação entre os modelos via DIC, $\widehat{E A I C}, \widehat{E B I C}$ e LMPL. . . . . . . . . . . . . 44

3.8 Estatísticas das distribuições marginais a posteriori dos parâmetros para o modelo

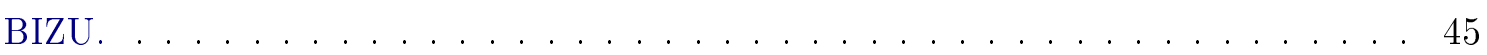

3.9 Estatísticas das distribuições marginais a posteriori dos parâmetros para o modelo Tobit normal inflacionado. . . . . . . . . . . . . . . . . . 45

3.10 Estatísticas das distribuições marginais a posteriori dos parâmetros para o modelo Tobit normal potência inflacionado. . . . . . . . . . . . . . . 45

3.11 Comparação entre os modelos via DIC, $\widehat{E A I C}, \widehat{E B I C}$ e LMPL. . . . . . . . . . . . 51

3.12 Estatísticas das distribuições marginais a posteriori dos parâmetros para o modelo

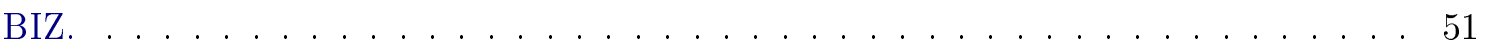

3.13 Estatísticas das distribuições marginais a posteriori dos parâmetros para o modelo Tobit normal inflacionado de zero. . . . . . . . . . . . . . 51

3.14 Estatísticas das distribuições marginais a posteriori dos parâmetros para o modelo tobit normal-potência inflacionado de zero. . . . . . . . . . . . . . . 51

6.1 Comparação entre as especificações do modelo Tobit segundo os critérios DIC e

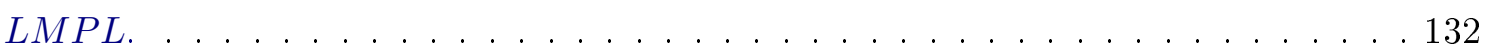

6.2 Comparação entre as especificações do modelo BIZU segundo os critérios DIC e LMPL． . . . . . . . . . . . . . . . . . . . . . . . . . . . . . . . . . . 132

6.3 Comparação entre os modelos Tobit, BIZU e Beta pelos critédios DIC, LMPL e métricas de predição. . . . . . . . . . . . . . . . . . . . 132

6.4 Comparação entre os modelos Tobit, BIZU e Beta pelo critédio DIC e MSE somente na previsão de 30 passos a frente. . . . . . . . . . . . . . . . . 132 


\section{Capítulo 1}

\section{Introdução}

A modelagem estatística para dados contínuos restritos a um intervalo limitado tem sido o foco de muitos estudos. Este interesse é ainda mais frequente na literatura para dados restritos ao intervalo $[0,1]$, no que se refere a conjuntos de dados que representam valores de taxas ou proporções. Alguns exemplos de dados contínuos de proporções incluem taxa de desemprego, mortalidade por acidentes de trânsito, fração de renda destinada ao fundo de aposentadoria, fração do valor de exportação da empresa no setor de indústria, etc. Modelos de regressão lineares e não lineares usuais não são adequados para este tipo de conjunto de dados. Algumas diferentes alternativas têm sido propostas para modelar proporções contínuas com a possibilidade de relacioná-las a variáveis exógenas, como o uso de transformações na variável resposta (vide Aitchison, 1982), censura na variável resposta ou assumindo uma distribuição para a variável resposta que está restrita a intervalos limitados. Os modelos de regressão Tobit e regressão beta são de interesse particular deste trabalho.

A distribuição beta tem despertado bastante interesse para modelagem em intervalos limitados devido a sua flexibilidade, uma vez que sua densidade pode assumir diferentes formas dependendo apenas dos dois parâmetros que caracterizam a distribuição (vide Johnson et al., 1995). Alguns modelos frequentistas de regressão beta foram propostos por Paolino (2001), Kieschnick e McCullough (2003), Ferrari e Cribari-Neto (2004) e Ospina e Ferrari (2012), entre outros. Na classe de modelos de regressão introduzidos por Ferrari e Cribari-Neto (2004), a condição básica assumida é que a variável resposta segue a distribuição onde o seu valor esperado está relacionado a um preditor linear por meio de uma função de ligação. Alguns modelos de regressão beta Bayesianos foram estudados por Branscum et al. (2007), Albi et al. (2009), Bayes et al. (2012), entre outros. Uma abordagem alternativa para modelagem de dados com estas características descritas acima é baseada em extensões dos modelos censurados Tobit proposto por Tobin (1958), também explorado por Mroz (1987), utilizando censura dupla nas extremidades do intervalo $[0,1]$. A versão Bayesiana do modelo Tobit foi estudada por Chib (1992).

Contudo, dados de proporções pode incluir uma quantidade não desprezível de valores extremos, zeros e/ou uns. Nesta situação, a distribuição beta em si não providencia uma descrição satisfatória dos dados, uma vez que ela não permite probabilidade positiva para nenhum ponto do intervalo $[0,1]$. Para contornar este problema, Ospina (2008) propôs uma versão com mistura contínua-discreta do modelo apresentado por Ferrari e Cribari-Neto (2004), utilizando a distribuição beta para definir a componente contínua da distribuição e a componente discreta é definida por uma Bernoulli ou uma distribuição degenerada em zero ou um.

Para o modelo Tobit duplamente censurado muitas vezes a censura não é o suficiente para contemplar a quantidades de valores extremos no conjunto de dados. Com isso, inflações de zeros e uns também podem ser consideradas utilizando a extensão do modelo de misturas apresentado por Cragg (1971), onde os valores extremos do intervalo são também definidos por uma Bernoulli ou uma distribuição degenerada. Os modelos de hurdle (McDowell, 2003) também podem ser inseridos neste contexto. Outra forma de utilizar a extensão do modelo proposto por Moulton e Halsey (1995), onde os valores extremos podem vir de uma componente discreta ou de uma censura da 
componente contínua. Em ambos os casos, é considerado que os excessos de zeros e/ou uns são provenientes de um modelo do tipo Bernoulli que liga os possíveis excessos de zeros e/ou uns a um grupo de covariáveis que podem influenciar a probabilidade de sua ocorrência e a resposta contínua é modelada utilizando a distribuição normal com função de ligação como utilizado usualmente na classe de modelos lineares generalizados. Extensões dos modelos Tobit duplamente censurados envolvendo assimetria, utilizando a classe de distribuições potência (Lehmann, 1953; Gupta e Gupta, 2008; Pewsey et al., 2012).

Neste trabalho desenvolvemos a versão Bayesiana dos modelos de regressão beta inflacionados de zero-e-um, propostos por Ospina (2008) sob a ótica frequentista e também a classe de modelos Bayesianos Tobit duplamente censurados e inflacionados de zero e um. As vantagens da análise Bayesiana são bem conhecidas e incluem a utilização de conhecimentos a priori, evitam o uso de aproximações assintóticas e a estimação prática de funções dos parâmetros por meio de técnicas de MCMC. Também discutimos algumas técnicas Bayesianas de diagnóstico como resíduos Bayesianos (Paolino et al., 2003) e medidas de influência baseadas na divergência-q como proposta por Peng e Dey (1995).

$\mathrm{Na}$ classe de dados em série de tempo, vale ressaltar que a abordagem Bayesiana de modelos dinâmicos têm se destacado como uma importante área da estatística nos últimos anos. Partindo da estrutura do modelo de regressão, esta abordagem se estende através da implementação de uma equação de evolução, capaz de governar a evolução temporal dos regressores. Esta classe é capaz de abranger muitos dos principais problemas envolvendo variações no tempo e espaço, inclusive os modelos de séries temporais.

A classe de modelos dinâmicos lineares é bastante abrangente. A extensão para os Modelos Dinâmicos Lineares Generalizados(MLDG) foi introduzida no trabalho de Migon (1984) e seguido por Migon e Harrison (1985), West et al. (1985), Gamerman e West (1987), Gamerman (1991), Gamerman (1998), Lindsey e Lambert (1995) e

Godolphin e Triantafyllopoulos (2006), os Modelos Lineares Dinâmicos (MLD) foram estendidos e generalizados em diversos contextos, sem a necessidade de impor a suposição de dados normalmente distribuídos.

No contexto de série de dados dinâmicos restritos no intervalo $[0,1]$ podemos citar alguns exemplos práticos em que há necessidade de sua modelagem e estudo de previsão. Análise do percentual de volume de água diário de um sistema de abastecimento é um exemplo em que os há presença de valores contínuos no intervalo $(0,1)$, como pode também ocorrer presença de valores extremos, 1 no caso de preenchimento total do volume e 0 em caso em que o volume chega a um valor abaixo do estado de alerta para o funcionamento do sistema de abastecimento. O percentual pago mensalmente da fatura de cartão de crédito por clientes de uma instituição financeira é outro exemplo da mesma estrutura de série temporal.

Introduziremos neste estudo extensões dos modelos dinâmico beta apresentados em da-Silva et al. (2011) e da-Silva e Migon (2012), utilizando a distribuição beta inflacionada de zero-e-um apresentada no contexto de regressão por Ospina (2008) e Ospina e Ferrari (2010). Também apresentaremos a classe de modelos Tobit duplamente censurado dinâmico como alternativa ao modelo beta para série de dados de taxas ou proporções. Dagenais (1982) e Zeger e Brookmeyer (1986) exploraram o modelo Tobit com autocorrelação nos erros, Lee (1997) desenvolveu a estimação do modelo dinâmico Tobit via métodos de simulação e a versão Bayesiana do modelo Tobit foi estudada por Wei (1999) com utilização de dados aumentados (Geman e Geman, 1984, Tanner e Wong, 1987 e Gelfand e Smith, 1990). O modelo Tobit duplamente censurado dinâmico desenvolvido neste trabalho se difere do modelo proposto por Wei (1999) por manter o aspecto de estimação sequencial da natureza dos modelos dinâmicos Bayesianos. Tanto o modelo dinâmico BIZU como o modelo dinâmico Tobit duplamente censurado foram formulados com estimação via a metodologia de filtro de partículas apresentada por Gordon et al. (1993) e estendido por Pitt e Shephard (1999). 


\subsection{Objetivos}

Este trabalho tem por objetivo construir uma ferramenta para a análise Bayesiana de dados em intervalos limitados. Isto inclui dados com a presença de valores extremos e mais especificamente, como principal foco do trabalho, dados de taxa ou proporção. Serão apresentadas metodologias para a análise de regressão linear, assim como técnicas Bayesianas para avaliar o seu ajuste.

No contexto de regressão linear, as contribuições deste trabalho são os modelos Bayesiano Beta inflacionado de zero e um, apresentado no contexto frequentista por Ospina (2008), o modelo Bayesiano Tobit duplamente censurado e inflacionado de zeros e uns, como extensão dos modelos estudados por Moulton e Halsey (1995) e Cragg (1971) sob a ótica frequentista, assim como a extensão do modelo Tobit normal-potência estudado por Gupta e Gupta (2008) e Pewsey et al. (2012).

Em um contexto de dados em série de tempo, é apresentada uma metodologia para analise de modelos dinâmicos para esta estrutura de dados. Apresentamos procedimentos capaz de construir análise de comportamento da série, assim como análise de previsão e análises retrospectivas em séries de dados em intervalos limitados, como o caso mais comum em $[0,1]$, podendo conter valores extremos.

Como contribuição na parte de modelos dinâmicos apresentamos o modelo dinâmico $B I Z U$, como extensão do modelo dinâmico Beta desenvolvido por da-Silva et al. (2011), e o modelo dinâmico Tobit duplamente censurado, como extensão do modelo dinâmico Tobit apresentado por Wei (1999), com estimação via filtro de partículas.

\subsection{Organização do Trabalho}

No Capítulo 2, apresentamos as distribuições candidatas para análise de dados de taxa ou proporção com presença não negligenciável de valores extremo, mais especificamente a distribuição Beta inflacionada de zeros e uns (BIZU) e o modelo tobit normal com algumas variações. No Capítulo 3, apresentamos as estruturas dos modelos de regressão Bayesiano, assim como metodologia Bayesiana de análise de ajuste e diagnóstico dos modelos. No Capítulo 4, é introduzida a classe de modelos dinâmicos e apresentada as estruturas dos modelos dinâmicos Beta inflacionado e Tobit. No Capítulo 5 é detalhada a técnica de filtro de partículas no contexto Bayesiano do modelos dinâmicos. No Capítulo 6 apresentamos estudos de simulação para estimação dos modelos dinâmicos BIZU e Tobit duplamente censurado e uma aplicação de dados reais. Por fim, o Capítulo 7 consiste em considerações finais e trabalhos futuros em continuidade deste estudo. 


\section{Capítulo 2}

\section{Mistura de distribuições}

Na literatura há alguns modelos conhecidos utilizando mistura de distribuições, principalmente aqueles que se encontram na classe de modelos inflacionados. O termo inflacionado sugere que a massa de probabilidade de alguns pontos excede o que é atribuído pelo modelo proposto. Alguns modelos conhecidos desta classe são: ZIP(Poisson inflacionado de zeros), ZIB (binomial inflacionada de zeros) e ZINB (binomial negativa inflacionada de zeros). Nesta seção apresentaremos um breve revisão de mistura de distribuições, uma vez que será necessário lidar com a classe de modelos inflacionados neste trabalho. Este capítulo tem como objetivo apresentar uma revisão das classes de distribuições que serão exploradas no desenvolvimento dos modelos Bayesianos.

\subsection{Caso geral}

Primeiramente vamos apresentar algumas propriedades da função de distribuição.

A função $F($.$) é uma função de distribuição se esta satisfaz:$

- $F($.$) é não decrescente;$

- $\lim _{x \rightarrow \infty} F(x)=1$ e $\lim _{x \rightarrow-\infty} F(x)=0$;

- $F($.$) é contínua a direita.$

Se $X$ é uma variável aleatória com $F($.$) como função de distribuição, então$

$$
F(x)-F(x-)=\operatorname{Pr}(X=x), \forall x .
$$

Onde $F(x-)=\lim _{\delta \downarrow 0} F(x-\delta)=\operatorname{Pr}(X \in(-\infty, x)), \quad \forall x$.

Vamos denotar $\mathcal{D}_{F}$ como o conjunto de descontinuidade da função de distribuição $F($.). Isto é,

$$
\mathcal{D}_{F}=\{x \mid F(x)-F(x-)>0\} .
$$

Pode-se mostrar que $\mathcal{D}_{F}$ é contável.

Agora vamos identificar três classes de variáveis aleatórias. As variáveis do tipo contínua e do tipo discreta são classes puras de variávels e a terceira classe será a classe do tipo de mistura (vide Kumar, 2004).

A variável aleatória será denominada do tipo contínua se sua função de distribuição é contínua, o que significa,

$$
\sum_{x \in \mathbb{R}}[F(x)-F(x-)]=0 .
$$

A variável aleatória será denominada do tipo discreta se sua função de distribuição é tal que,

$$
\sum_{x \in \mathbb{R}}[F(x)-F(x-)]=1 .
$$


A variável aleatória será denominada do tipo de mistura se sua função de distribuição é tal que,

$$
0<\sum_{x \in \mathbb{R}}[F(x)-F(x-)]<1 .
$$

Considerando $F$ uma função de distribuição do tipo de mistura e $X \sim F$. Note que $\mathcal{D}_{F}$ é não vazio e $\operatorname{Pr}\left(x \in \mathcal{D}_{F}\right) \in(0,1)$. Seja $F_{D}(x)=\operatorname{Pr}\left(X \leq x \mid X \in \mathcal{D}_{F}\right)$ e $F_{C}(x)=\operatorname{Pr}\left(X \leq x \mid X \in \mathcal{D}_{F}^{c}\right)$, $\forall x \in \mathbb{R}$. Também, considere $X_{D}$ e $X_{C}$ variáveis aleatórias com $F_{D}($.$) e F_{C}($.$) como suas funções de$ distribuição, respectivamente. Pelo teorema de Bayes, então

$$
F(x)=p_{D} \times F_{D}+p_{C} \times F_{C}, \quad \forall x \in \mathbb{R} .
$$

Analisando $F_{D}($.$) , observe que$

$$
\begin{aligned}
\operatorname{Pr}\left(\left\{X_{D}=x\right\}\right) & =\operatorname{Pr}\left(X=x \mid X \in \mathcal{D}_{F}\right)=\frac{\operatorname{Pr}\left(\{X=x\} \cap\left\{X \in \mathcal{D}_{F}\right\}\right)}{\operatorname{Pr}\left(X \in \mathcal{D}_{F}\right)} \\
& =\frac{\operatorname{Pr}(\{X=x\})}{p_{D}} .
\end{aligned}
$$

Também, note que

$$
F_{C}=\frac{F(.)-p_{D} F_{D}(.)}{p_{C}}, \quad p_{C}>0 .
$$

Se todos os momentos de interesse existem, então

$$
\begin{aligned}
E\left(X^{k}\right) & =p_{D} \times E\left(X_{D}^{k}\right)+p_{C} \times E\left(X_{C}^{k}\right), \quad \forall k ; \\
\operatorname{Var}(X) & =\left(p_{D} \times \operatorname{Var}\left(X_{D}\right)+p_{C} \times \operatorname{Var}\left(X_{C}\right)\right)+p_{D} \times p_{C} \times\left(E\left(X_{D}\right)-E\left(X_{C}\right)\right)^{2} .
\end{aligned}
$$

\subsection{Distribuição Beta inflacionada de zeros e/ou uns}

Considere a distribuição beta com parâmetros $\mu$ e $\phi$, onde $0<\mu<1$ e $\phi>0$, denotada por $\mathcal{B}(\mu, \phi)$, esta possui a seguinte função de densidade

$$
f(y ; \mu, \phi)=\frac{\Gamma(\phi)}{\Gamma(\mu \phi) \Gamma((1-\mu) \phi)} y^{\mu \phi-1}(1-y)^{(1-\mu) \phi-1}, \quad y \in(0,1),
$$

onde $\Gamma$ (.) é a função gama. A parametrização em (2.1) foi apresentada por Ferrari e Cribari-Neto (2004) e é uma forma mais confortável para a modelagem. Se $y \sim \mathcal{B}(\mu, \phi)$, então $E(Y)=\mu$ e $\operatorname{Var}(Y)=\mu(1-\mu) /(\phi+1)$ e $\phi$ pode ser interpretado como o parâmetro de precisão. A distribuição beta é bastante atraente para modelagem de dados contínuos em $(0,1)$ devido a sua grande flexibilidade de formas em sua densidade, como simétrica, assimétrica a esquerda e a direita, forma de 'U', ' $J$ ' e ' $J$ ' invertido (vide Figura (2.1)). Entretanto, todas as suas formas possíveis possui a restrição descrita no teorema a seguir:

Teorema 1 Dado uma variável aleatória $y \sim \operatorname{Beta}(\mu, \phi)$ :

- Se $\mu \phi>1$ então $y \rightarrow 0 \Rightarrow f(y) \rightarrow 0$;

- Se $\mu \phi<1$ então $y \rightarrow 0 \Rightarrow f(y) \rightarrow \infty$;

- Se $(1-\mu) \phi>1$ então $y \rightarrow 1 \Rightarrow f(y) \rightarrow 0$;

- Se $(1-\mu) \phi<1$ então $y \rightarrow 1 \Rightarrow f(y) \rightarrow \infty$.

Além disso, a distribuição beta não é apropriada para modelar conjunto de dados que possuem valores iguais a zero ou um. Para contornar esta restrição, Ospina (2008) sugeriu um modelo utilizando a mistura de duas distribuições: a distribuição beta e uma Bernoulli, que atribui probabilidade não 
negativa para os valores 0 e 1 . Neste modelo, a distribuição beta é utilizada para modelar a componente contínua dos dados e a distribuição Bernoulli ajusta a componente discreta. Ospina (2008) denominou a distribuição beta inflacionada de zeros ou um como $B I c, c \in\{0,1\}$, e a distribuição beta inflacionada de zeros e uns como BIZU. Estas distribuições podem ser expressas como uma combinação convexa de duas funções de distribuição acumulada como

$$
B I c(y ; \alpha, \mu, \phi)=\alpha \mathbb{1}_{\{c\}}+(1-\alpha) F(y ; \mu, \phi),
$$

onde $\alpha=P(Y=c)$ e $F(y ; \mu, \phi)$ representa a função de distribuição acumulada de $\mathcal{B}(\mu, \phi)$ e

$$
B I Z U(y ; \alpha, \gamma, \mu, \phi)=\alpha \operatorname{Ber}(y ; \gamma)+(1-\alpha) F(y ; \mu, \phi),
$$

onde $\operatorname{Ber}(y ; \gamma)$ representa a função de distribuição acumulada de uma variável aleatória Bernoulli com parâmetro $\gamma$ e $F(y ; \mu, \phi)$ representa a função de distribuição acumulada de $\mathcal{B}(\mu, \phi)$. O parâmetro de mistura $\alpha$, com $0<\alpha<1$, permite a combinação convexa entre as duas funções de distribuição.

A função de verossimilhança da variável com distribuição $B I_{c}$ é da forma

$$
b i_{c}(y ; \alpha, \mu, \phi)= \begin{cases}\alpha, & \text { se } y=c, \\ (1-\alpha) f(y ; \mu, \phi) & \text { se } y \in(0,1)\end{cases}
$$

onde $f(y ; \mu, \phi)$ corresponde a função de densidade beta (2.1) e neste caso denotamos $y \sim B I_{c}(\alpha, \mu, \phi)$. Vale ressaltar que $\alpha=P(Y=c)$, onde $c=0$ ou $c=1$. Para obter os momentos de interesse de $Y$, utilizamos as regras dos momentos condicionais, com $E(Y)=E\left[E\left(Y \mid \mathbb{1}_{\{c\}}\right)\right]$ e $\operatorname{Var}(Y)=$ $E\left[\operatorname{Var}\left(Y \mid \mathbb{1}_{\{c\}}\right)\right]+\operatorname{Var}\left[E\left(Y \mid \mathbb{1}_{\{c\}}\right)\right]$, onde

$$
\begin{aligned}
E\left[Y \mid \mathbb{1}_{\{c\}}(y)\right] & = \begin{cases}c, & \text { com probabilidade } \alpha, \\
\mu, & \text { com probabilidade } 1-\alpha,\end{cases} \\
\operatorname{Var}\left[Y \mid \mathbb{1}_{\{c\}}(y)\right] & = \begin{cases}0, & \text { com probabilidade } \alpha, \\
\frac{\mu(1-\mu)}{\phi+1}, & \text { com probabilidade } 1-\alpha,\end{cases}
\end{aligned}
$$

Porantanto, a média e a variância de $Y$ podem ser descritas da seguinte forma:

$$
\begin{aligned}
E[Y] & =\alpha c+(1-\alpha) \mu \\
\operatorname{Var}[Y] & =(1-\alpha) \frac{\mu(1-\mu)}{\phi+1}+\alpha(1-\alpha)(\mu)^{2} .
\end{aligned}
$$

A função de verossimilhança da variável com distribuição $B I Z U$ é da forma

$$
\text { bizu }(y ; \alpha, \gamma, \mu, \phi)= \begin{cases}\alpha \gamma, & \text { se } y=1, \\ \alpha(1-\gamma), & \text { se } y=0, \\ (1-\alpha) f(y ; \mu, \phi) & \text { se } y \in(0,1),\end{cases}
$$

onde $f(y ; \mu, \phi)$ corresponde a função de densidade beta (2.1) e neste caso denotamos $y \sim B I Z U(\alpha, \gamma, \mu, \phi)$. Note que $P(Y=1)=\alpha \gamma$ e $P(Y=0)=\alpha(1-\gamma)$ e para $0<a<b<1$,

$$
P(Y \in(a, b))=(1-\alpha) \int_{a}^{b} f(y ; \mu, \phi) d y .
$$


Da mesma forma, os momentos de interesse são dados por

$$
\begin{aligned}
E\left[Y \mid \mathbb{1}_{\{0,1\}}(y)\right] & = \begin{cases}\gamma, & \text { com probabilidade } \alpha, \\
\mu, & \text { com probabilidade } 1-\alpha,\end{cases} \\
\operatorname{Var}\left[Y \mid \mathbb{1}_{\{0,1\}}(y)\right] & = \begin{cases}\gamma(1-\gamma), & \text { com probabilidade } \alpha, \\
\frac{\mu(1-\mu)}{\phi+1}, & \text { com probabilidade } 1-\alpha,\end{cases}
\end{aligned}
$$

Porantanto, a média e a variância de $y$ podem ser descritas da seguinte forma:

$$
\begin{aligned}
E[Y] & =\alpha \gamma+(1-\alpha) \mu, \\
\operatorname{Var}[Y] & =\alpha \gamma(1-\gamma)+(1-\alpha) \frac{\mu(1-\mu)}{\phi+1}+\alpha(1-\alpha)(\gamma-\mu)^{2} .
\end{aligned}
$$

Um exemplo das curvas das funções de distribuição acumulada e das funções de densidade das variáveis $B I c$ e $B I Z U$ podem ser observadas nas Figuras (2.2) e (2.3).
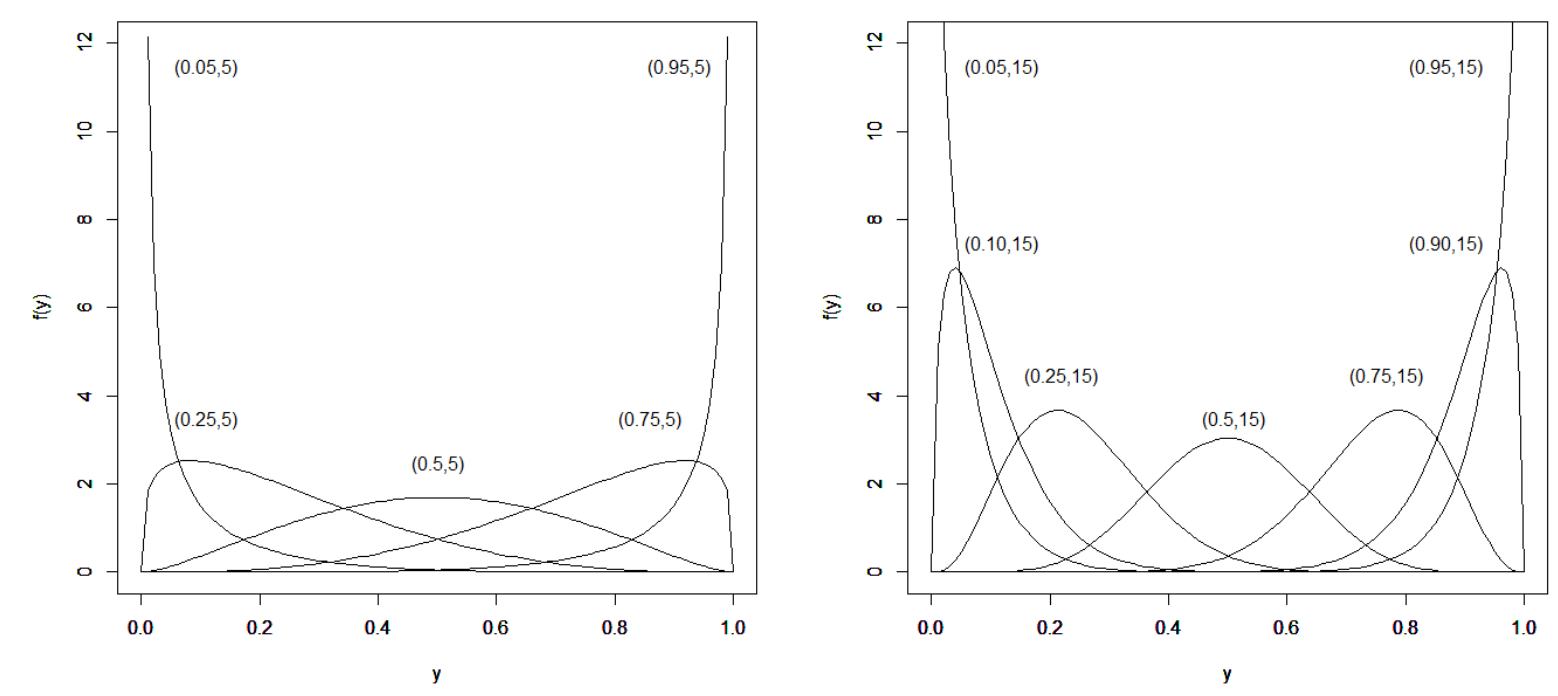

Figura 2.1: Gráfico das densidades beta para diferentes valores de $(\mu, \phi)$. 

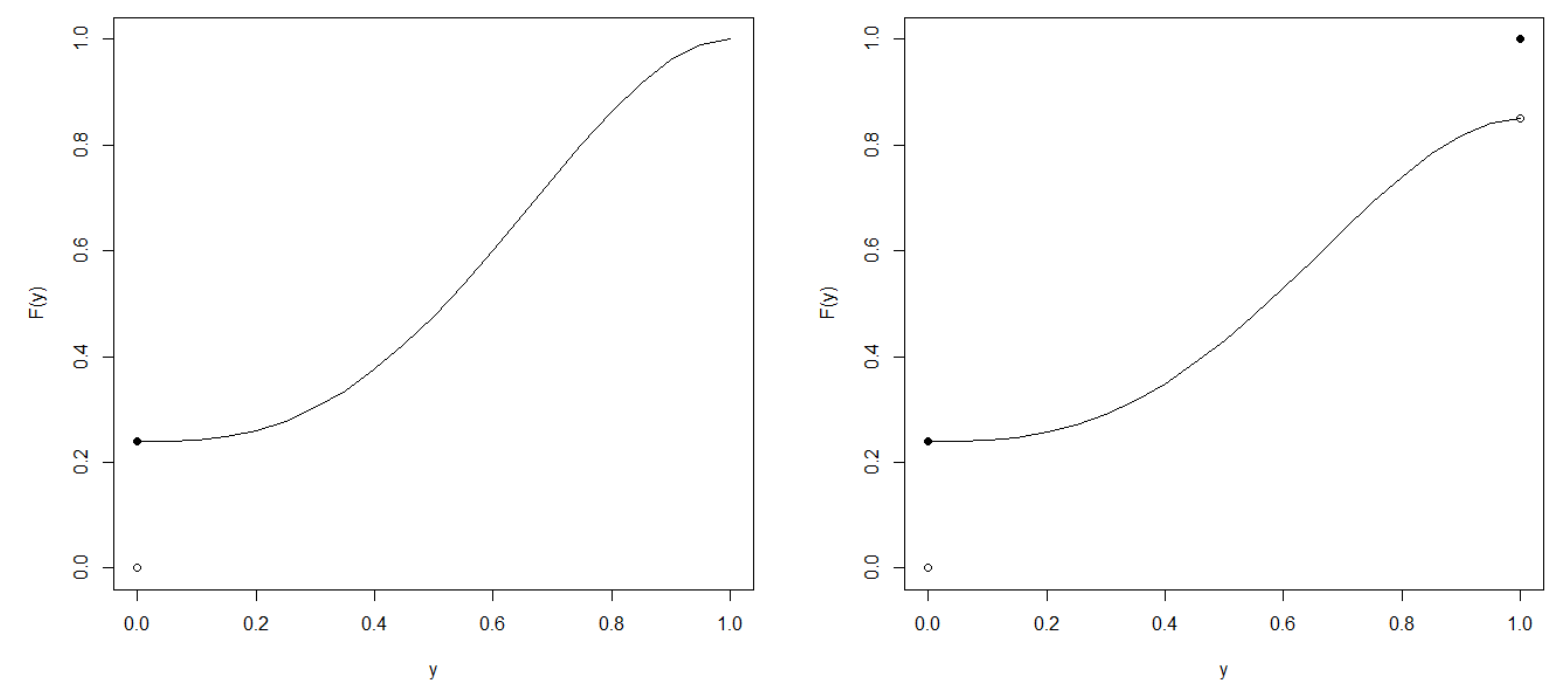

Figura 2.2: Gráfico das funções de distribuição acumulada das variáveis $B I_{0}$ (esquerda) e BIZU (direita), considerando os parâmetros $(\alpha=0.24, \mu=0.6, \phi=5)$ e $(\alpha=0.39, \gamma=0.38, \mu=0.6, \phi=5)$, respectivamente.
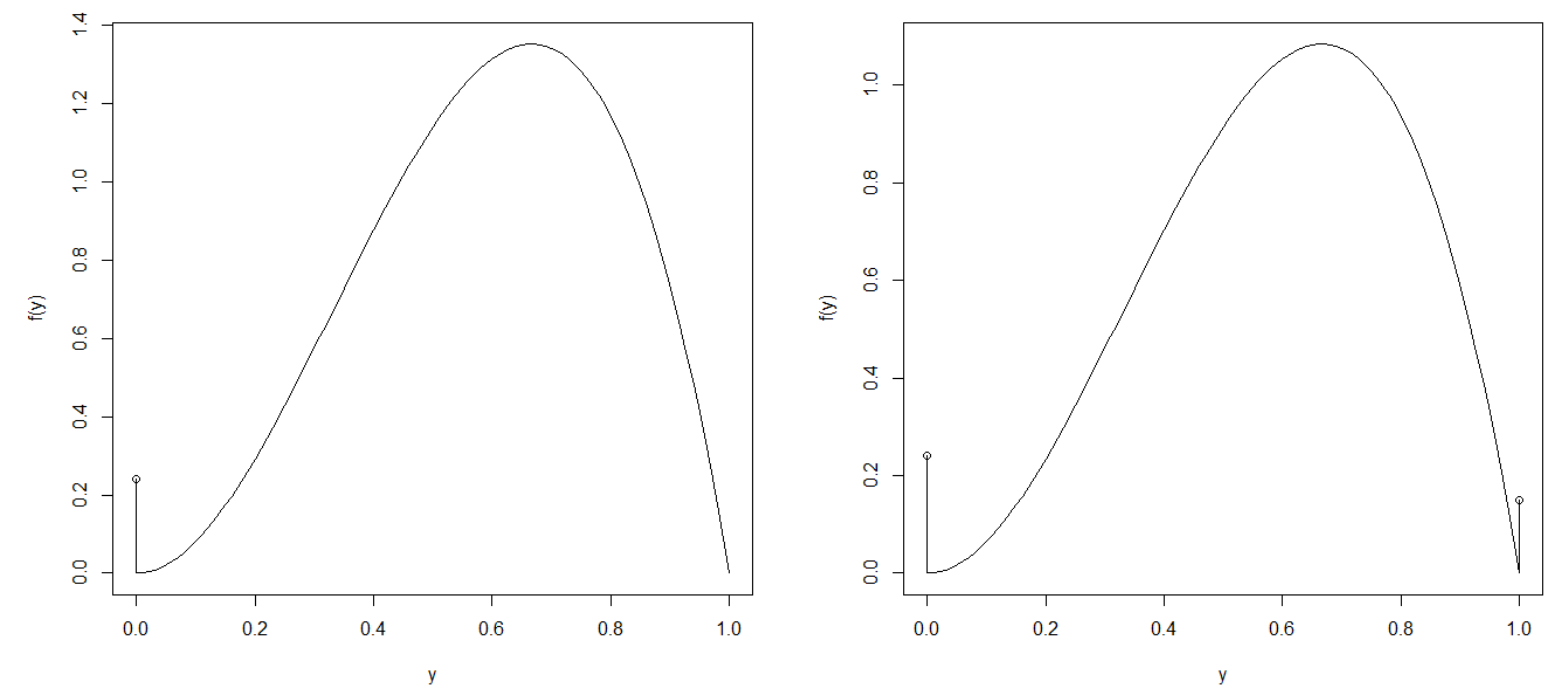

Figura 2.3: Gráfico das funções de verossimilhança das variáveis $B I_{0}$ (esquerda) e BIZU (direita), considerando os parâmetros $(\alpha=0.24, \mu=0.6, \phi=5)$ e $(\alpha=0.39, \gamma=0.38, \mu=0.6, \phi=5)$, respectivamente. 


\subsection{Distribuição Tobit normal duplamente censurada e inflacionada de zeros e/ou uns}

Estendendo a a idéia popularizada por Tobin (1958) em utilizar os zeros, ou qualquer outro ponto $c$ que represente o valor mínimo do conjunto de dados, como observações latentes e que resultou no modelo Tobit, formulado como

$$
y= \begin{cases}c & \text { se } w \leq c \\ w & \text { se } w>c\end{cases}
$$

onde a variável latente $w$ segue uma distribuição $N\left(\mu, \sigma^{2}\right)$. Neste caso, denominamos que os dados apresentam censura a esquerda e temos que $P(Y=c)=P(W \leq c)=\Phi\left(\frac{c-\mu}{\sigma}\right)$, onde $\Phi($.$) representa$ a função de distribuição acumulada da normal padrão.

Neste caso, a função de distribuição acumulada da variável $y$ correspondente ao modelo Tobit normal é dada por

$$
F(y)= \begin{cases}0, & \text { se } y<c \\ \Phi\left(\frac{y-\mu}{\sigma}\right), & \text { se } y \geq c .\end{cases}
$$

A função de verossimilhança de uma observação da variável $Y$ correspondente ao modelo Tobit normal é dada por

$$
f(y)=\left[\frac{1}{\sigma} \phi\left(\frac{y-\mu}{\sigma}\right)\right]^{1-I_{c}}\left[\Phi\left(\frac{c-\mu}{\sigma}\right)\right]^{I_{c}}
$$

onde

$$
I_{c}= \begin{cases}0, & \text { se } y>c, \\ 1, & \text { se } y=c .\end{cases}
$$

O caso do Tobit normal duplamente censurado segue a mesma analogia do modelo introduzido por Tobin (1958), acrescentando que agora temos censura a direita e a esquerda. A variável observada $y$ é descrita pela variável latente $w$ e os pontos de censura a esquerda $c_{1}$ e a direita $c_{2}$, seguindo a forma

$$
y= \begin{cases}c_{1} & \text { se } w \leq c_{1} \\ w & \text { se } c_{1}<w<c_{2} \\ c_{2} & \text { se } w \geq c_{2}\end{cases}
$$

onde a variável latente $w$ segue uma distribuição $N\left(\mu, \sigma^{2}\right)$. Temos que $P\left(Y=c_{1}\right)=P\left(W \leq c_{1}\right)=$ $\Phi\left(\frac{c_{1}-\mu}{\sigma}\right)$ e $P\left(Y=c_{2}\right)=P\left(W \geq c_{2}\right)=1-\Phi\left(\frac{c_{2}-\mu}{\sigma}\right)$, onde $\Phi($.$) representa a função de distribuição$ acumulada da normal padrão. Sem perda de generalidade, vamos considerar $c_{1}=0$ e $c_{2}=1$ para inserir o modelo no contexto de dados de taxas ou proporções contínuas.

Neste caso, a função de distribuição acumulada da variável $y$ correspondente ao modelo Tobit normal é dada por

$$
F(y)= \begin{cases}0, & \text { se } y<0 \\ \Phi\left(\frac{y-\mu}{\sigma}\right), & \text { se } 0 \leq y<1 \\ 1, & \text { se } y \geq 1\end{cases}
$$

A função verossimilhança de uma observação da variável $y$ correspondente ao modelo tobit normal é dada por

$$
f(y)=\left[\Phi\left(\frac{-\mu}{\sigma}\right)\right]^{I_{0}}\left[1-\Phi\left(\frac{1-\mu}{\sigma}\right)\right]^{I_{1}}\left[\frac{1}{\sigma} \phi\left(\frac{y-\mu}{\sigma}\right)\right]^{1-I_{0}-I_{1}}
$$


onde $0 \leq y \leq 1, \sigma>0, \mu \in \mathbb{R}$ e as variáveis auxiliares $I_{0}$ e $I_{1}$ são da forma

$$
I_{0}= \begin{cases}1, & \text { se } y=0, \\ 0, & \text { caso contrário }\end{cases}
$$

e

$$
I_{1}= \begin{cases}1, & \text { se } y=1, \\ 0, & \text { caso contrário. }\end{cases}
$$

Seguindo no contexto de modelos inflacionados, muitas vezes a quantidade observada de valores extremos excede a quantidade que pode ser representada pelo modelo Tobit e o uso de mistura da distribuição do modelo Tobit com uma distribuição Bernoulli ou degenerada para os valores extremos é uma forma eficiente de contornar este problema. Adaptando o modelo proposto por Moulton e Halsey (1995), temos o modelo Tobit duplamente censurado.

Com probabilidade $\delta=\delta_{0}+\delta_{1}, Y \sim \operatorname{Ber}\left(\delta_{1} / \delta\right), 0<\delta<1$ e com probabilidade $1-\delta, Y$ segue o modelo Tobit duplamente censurado da forma:

$$
y= \begin{cases}0 & \text { se } w \leq 0 \\ w & \text { se } 0<w<1 \\ 1 & \text { se } w \geq 1\end{cases}
$$

onde $W$ corresponde a variável latente do modelo Tobit e no caso Tobit normal temos $W \sim N\left(\mu, \sigma^{2}\right)$. Desta forma, temos que

$$
\begin{aligned}
& P(Y=0)=\delta_{0}+(1-\delta) \Phi\left(\frac{-\mu}{\sigma}\right) \\
& P(Y=1)=\delta_{1}+(1-\delta)\left[1-\Phi\left(\frac{1-\mu}{\sigma}\right)\right],
\end{aligned}
$$

e para $0<a<b<1$ temos

$$
P(Y \in(a, b))=(1-\delta)\left[\Phi\left(\frac{b-\mu}{\sigma}\right)-\Phi\left(\frac{a-\mu}{\sigma}\right)\right] .
$$

Com as probabilidades $\delta_{0}$ e $\delta_{1}$ são desconhecidas. Desta forma, a função de distribuição acumulada é dada por

$$
F(y)= \begin{cases}0, & \text { se } y<0 \\ \delta_{0}+(1-\delta) \Phi\left(\frac{y-\mu}{\sigma}\right), & \text { se } 0 \leq y<1 \\ 1, & \text { se } y \geq 1\end{cases}
$$

A função de verossimilhança de uma observação da variável $y$ correspondente ao modelo Tobit normal inflacionado é dada por

$$
\begin{aligned}
f(y)= & \left\{\delta_{0}+(1-\delta) \Phi\left(\frac{-\mu}{\sigma}\right)\right\}^{I_{0}}\left\{\delta_{1}+(1-\delta)\left[1-\Phi\left(\frac{1-\mu}{\sigma}\right)\right]\right\}^{I_{1}} \\
& \times\left\{(1-\delta) \frac{1}{\sigma} \phi\left(\frac{y-\mu}{\sigma}\right)\right\}^{1-I_{0}-I_{1}}
\end{aligned}
$$

onde $0 \leq y \leq 1, \sigma>0, \mu \in \mathbb{R}$ e as variáveis auxiliares $I_{0}$ e $I_{1}$ são da mesma forma descrita anteriormente.

Para obter os momentos de interesse do modelo, vamos considerar $\alpha$ como a probabilidade de 
$Y \in\{0,1\}$, ou seja:

$$
\alpha=\delta_{0}+\delta_{1}+(1-\delta)\left(\Phi\left(\frac{-\mu}{\sigma}\right)+1-\Phi\left(\frac{1-\mu}{\sigma}\right)\right) .
$$

E ainda considere $\gamma=P(Y=1 \mid y \in\{0,1\})$, ou seja

$$
\gamma=\frac{\delta_{1}+(1-\delta)\left(1-\Phi\left(\frac{1-\mu}{\sigma}\right)\right)}{\alpha} .
$$

Com isso, temos que $P(Y=1)=\alpha \gamma$ e $P(Y=0)=\alpha(1-\gamma)$.

Considerando inicialmente apenas componente contínua da variável $Y$, temos que:

$$
\begin{aligned}
E[Y \mid y \in(0,1)] & =\mu_{c}=\frac{1}{\Phi\left(\frac{1-\mu}{\sigma}\right)-\Phi\left(-\frac{\mu}{\sigma}\right)} \int_{0}^{1} y_{i} \frac{1}{\sigma} \phi\left(\frac{y-\mu}{\sigma}\right) d y \\
& =\frac{\sigma}{\sqrt{2 \pi}} \frac{e^{-a}-e^{-b}}{\Phi\left(\frac{1-\mu}{\sigma}\right)-\Phi\left(-\frac{\mu}{\sigma}\right)}+\mu,
\end{aligned}
$$

onde $a=\frac{\mu^{2}}{2 \sigma^{2}}$ e $b=\frac{(1-\mu)^{2}}{2 \sigma^{2}}$. Da mesma forma, temos que:

$$
\begin{aligned}
E\left[Y^{2} \mid y \in(0,1)\right] & =\frac{1}{\Phi\left(\frac{1-\mu}{\sigma}\right)-\Phi\left(-\frac{\mu}{\sigma}\right)} \int_{0}^{1} y^{2} \frac{1}{\sigma} \phi\left(\frac{y-\mu}{\sigma}\right) d y \\
& =\frac{\sigma^{2} D}{\Phi\left(\frac{1-\mu}{\sigma}\right)-\Phi\left(-\frac{\mu}{\sigma}\right)}+2 \mu\left(\mu_{c}-\mu\right)+\mu^{2},
\end{aligned}
$$

onde

$$
\gamma(h, u)=\int_{0}^{u} t^{h-1} e^{-t} d t
$$

e

$$
D=\frac{1}{\sqrt{\pi}}[\gamma(3 / 2, b)+\gamma(3 / 2, a)]
$$

Por fim,

$$
\begin{aligned}
\operatorname{Var}[Y \mid y \in(0,1)] & =E\left[Y^{2} \mid y \in(0,1)\right]-E[Y \mid y \in(0,1)] \\
& =\sigma_{c}^{2} .
\end{aligned}
$$

Seja $\mathbb{1}_{\{0,1\}}(y)$ a variável indicadora se $y \in\{0,1\}$, ou seja, se $y$ pertence a componente discreta, temos a média e variância condicionais de $y$ dadas por

$$
\begin{aligned}
E\left[Y \mid \mathbb{1}_{\{0,1\}}(y)\right] & = \begin{cases}\gamma, & \text { com probabilidade } \alpha, \\
\mu_{c}, & \text { com probabilidade } 1-\alpha,\end{cases} \\
\operatorname{Var}\left[Y \mid \mathbb{1}_{\{0,1\}}\left(y_{i}\right)\right] & = \begin{cases}\gamma(1-\gamma), & \text { com probabilidade } \alpha, \\
\sigma_{c}^{2}, & \text { com probabilidade } 1-\alpha,\end{cases}
\end{aligned}
$$


Porantanto, a média e a variância de $y$ podem ser descritas da seguinte forma:

$$
\begin{aligned}
E[Y] & =\alpha \gamma+(1-\alpha) \mu_{c}, \\
\operatorname{Var}[Y] & =\alpha \gamma(1-\gamma)+(1-\alpha) \sigma_{c}^{2}+\alpha(1-\alpha)\left(\gamma-\mu_{c}\right)^{2} .
\end{aligned}
$$

Para o caso do tobit normal inflacionado de zeros, no lugar da variável $y$ assumir com probabilidade $\delta$ uma distribuição $\operatorname{Ber}\left(\delta_{1} / \delta\right)$, tome $\delta_{1}=0$, ou seja $\delta=\delta_{0}$ e para o tobit normal inflacionado de uns tome $\delta_{0}=0, \delta=\delta_{1}$. Pode-se utilizar uma outra abordagem do modelo tobit duplamente censurado adaptando o modelo apresentado por Cragg (1971), onde é considerado que os valores extremos são representados simplesmente pela componente de inflação do modelo, neste caso temos a função de distribuição acumulada representada por

$$
F(y)= \begin{cases}0, & \text { se } y<0 \\ \delta_{0}+(1-\delta) \frac{\Phi\left(\frac{y-\mu}{\sigma}\right)}{\Phi\left(\frac{1-\mu}{\sigma}\right)-\Phi\left(\frac{-\mu}{\sigma}\right)}, & \text { se } 0 \leq y<1 \\ 1, & \text { se } y \geq 1 .\end{cases}
$$

A função de verossimilhança de uma observação da variável $Y$ correspondente ao modelo Tobit normal inflacionado é dada por

$$
f(y)=\left\{\delta_{0}\right\}^{I_{0}}\left\{\delta_{1}\right\}^{I_{1}}\left\{(1-\delta) \frac{\frac{1}{\sigma} \phi\left(\frac{y-\mu}{\sigma}\right)}{\Phi\left(\frac{1-\mu}{\sigma}\right)-\Phi\left(\frac{-\mu}{\sigma}\right)}\right\}^{1-I_{0}-I_{1}} .
$$

E para o cálculo dos momentos basta tomar $\alpha=\delta$ e $\gamma=\delta_{1} / \delta$.

É importante notar que apesar da distribuição normal não ser tão flexível em relação as formas que sua densidade pode apresentar, como a distribuição beta, mas no caso da distribuição normal duplamente censurada ou truncada é possível atribuir diferentes formas para sua componente contínua a partir de deslocamentos na moda e mudanças em sua variância. A Figura (2.4) ilustra algumas possíveis formas que a distribuição normal pode apresentar no intervalo $[0,1]$ para diferentes valores de $\mu$ e $\sigma$.

Um exemplo das curvas das funções de distribuição acumulada e das funções de densidade das variáveis tobit normal inflacionada de zeros e tobit normal inflacionada de zeros e uns podem ser observadas nas Figuras (2.8) e (2.9). 

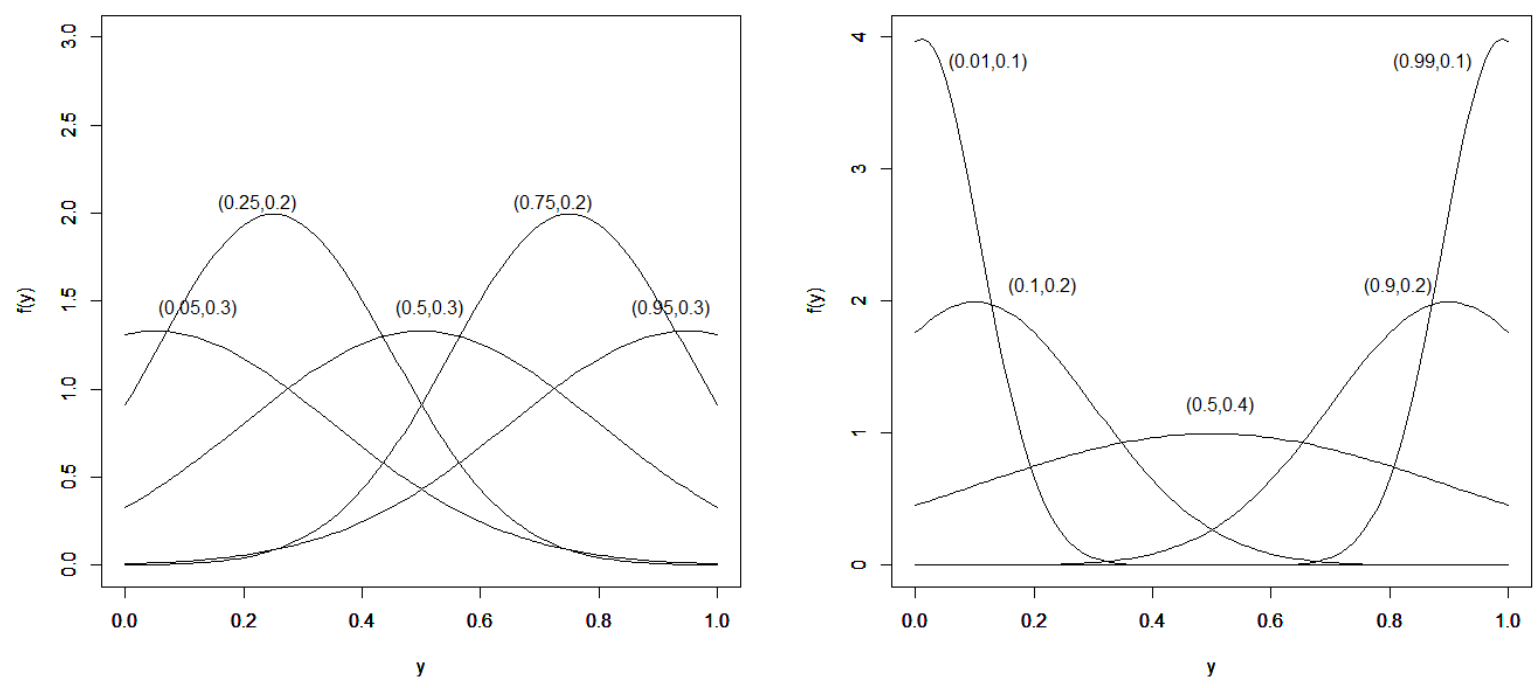

Figura 2.4: Gráfico das densidades Tobit normal inflacionada para diferentes valores de $(\mu, \sigma)$.
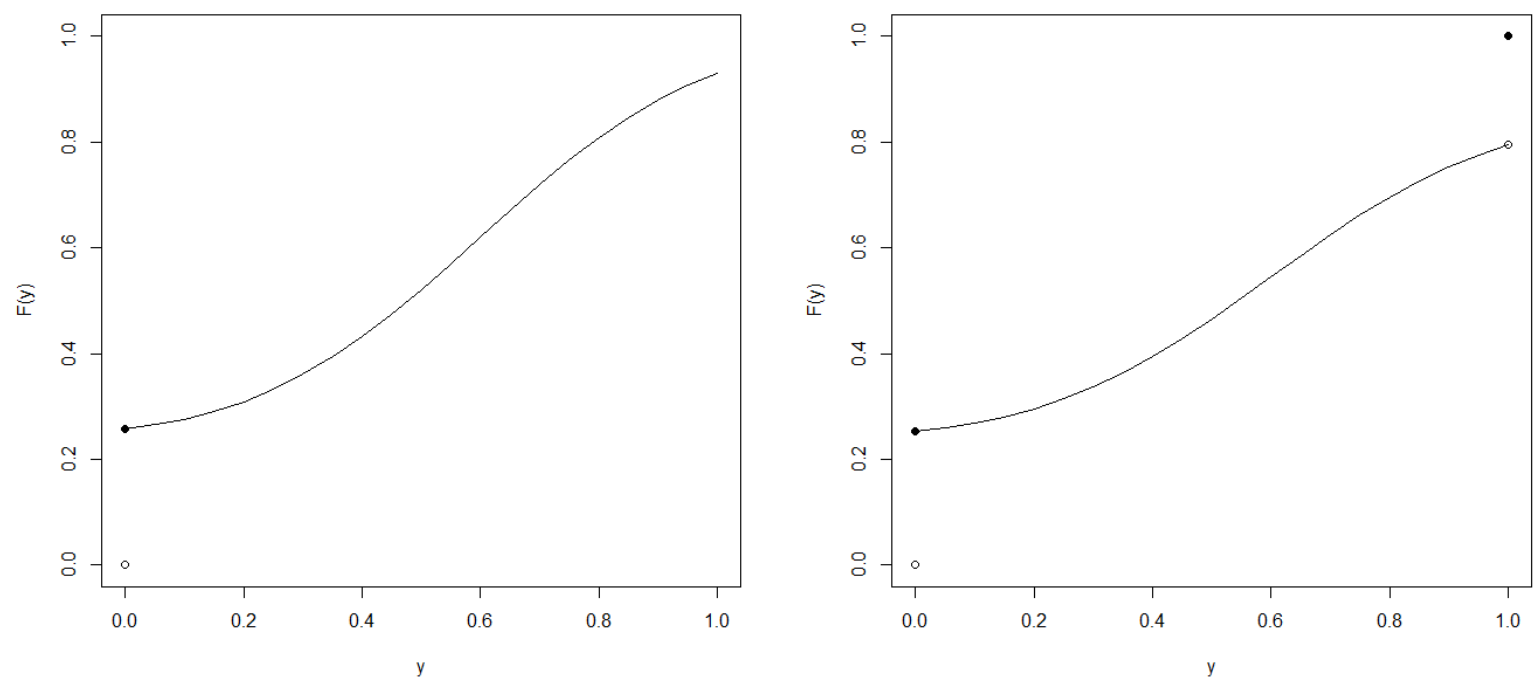

Figura 2.5: Gráfico das funçôes de distribuição acumulada das variáveis tobit normal inflacionada de zeros (esquerda) e tobit normal inflacionada de zeros e uns (direita), considerando os parâmetros $(\delta=0.24, \mu=$ $0.6, \sigma=0.3)$ e $\left(\delta_{0}=0.24, \delta_{1}=0.15, \mu=0.6, \sigma=0.3\right)$, respectivamente. 
DISTRIBUIÇÃO TOBIT NORMAL DUPLAMENTE CENSURADA E INFLACIONADA DE ZEROS E/OU UNS
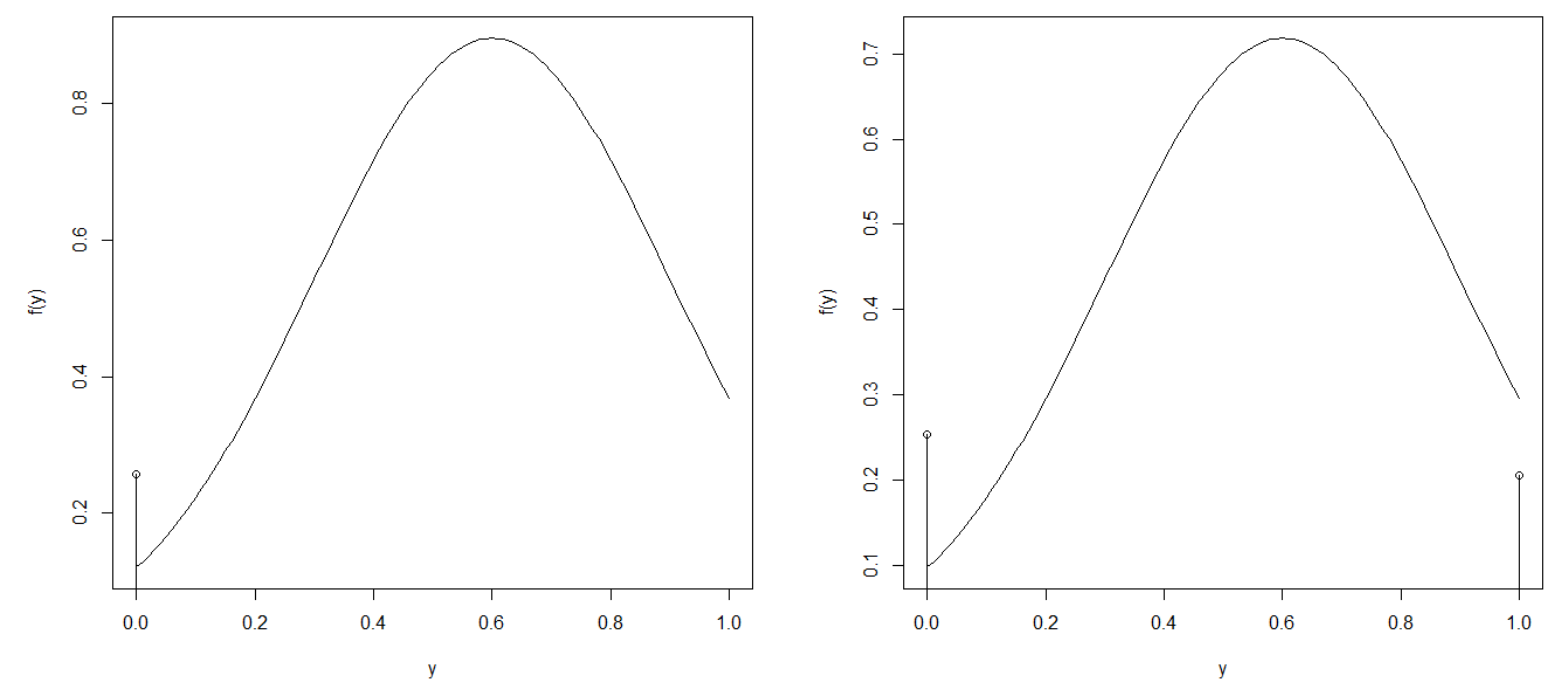

Figura 2.6: Gráfico das funções de verossimilhança das variáveis Tobit normal inflacionada de zeros (esquerda) e tobit normal inflacionada de zeros e uns (direita), considerando os parâmetros $(\delta=0.24, \mu=$ $0.6, \sigma=0.3) e\left(\delta_{0}=0.24, \delta_{1}=0.15, \mu=0.6, \sigma=0.3\right)$, respectivamente. 


\subsection{Distribuição Tobit normal-potência duplamente censurada e in- flacionada de zeros e/ou uns}

Com o intuito de aumentar a flexibilidade do modelo Tobit normal incorporando assimetria, utilizamos o modelo Tobit normal-potência, onde a variável latente do modelo Tobit segue uma distribuição $N P\left(\alpha, \mu, \sigma^{2}\right)$ da classe de distribuições potência introduzida por Lehmann (1953) e explorada por Gupta e Gupta (2008) e Pewsey et al. (2012). Neste caso, tomando $W \sim N P\left(\alpha, \mu, \sigma^{2}\right)$ temos que sua função de distribuição acumulada é dada por

$$
F(w)=\Phi\left(\frac{w-\mu}{\sigma}\right)^{\alpha}, \quad \mu, w \in \mathbb{R}, \quad \alpha, \sigma \in \mathbb{R}^{+},
$$

onde $\Phi($.$) corresponde a função de distribuição acumulada da normal padrão. Derivando a função$ acima obtemos a função de densidade $N P\left(\alpha, \mu, \sigma^{2}\right)$, dada por

$$
f(w)=\frac{\alpha}{\sigma} \phi\left(\frac{w-\mu}{\sigma}\right)\left[\Phi\left(\frac{w-\mu}{\sigma}\right)\right]^{\alpha-1}, \quad \mu, w \in \mathbb{R}, \quad \alpha, \sigma \in \mathbb{R}^{+} .
$$

No contexto dos modelos Tobit duplamente censurado e inflacionado, utilizamos a mesma estrutura do caso do modelo tobit normal, de modo que $Y \sim \operatorname{Ber}\left(\delta_{1} / \delta\right)$ com probabilidade $\delta=\delta_{0}+\delta_{1}$, $0<\delta<1$ e com probabilidade $1-\delta, y$ segue o modelo tobit duplamente censurado da forma:

$$
y= \begin{cases}0 & \text { se } w \leq 0 \\ w & \text { se } 0<w<1 \\ 1 & \text { se } w \geq 1\end{cases}
$$

onde $W$ corresponde a variável latente do modelo tobit e no caso tobit normal temos $W \sim$ $N P\left(\alpha, \mu, \sigma^{2}\right)$. Desta forma, temos que

$$
\begin{aligned}
& P(Y=0)=\delta_{0}+(1-\delta) \Phi\left(\frac{-\mu}{\sigma}\right)^{\alpha} \\
& P(Y=1)=\delta_{1}+(1-\delta)\left[1-\Phi\left(\frac{1-\mu}{\sigma}\right)^{\alpha}\right]
\end{aligned}
$$

e para $0<a<b<1$ temos

$$
P(Y \in(a, b))=(1-\delta)\left[\Phi\left(\frac{b-\mu}{\sigma}\right)^{\alpha}-\Phi\left(\frac{a-\mu}{\sigma}\right)^{\alpha}\right]
$$

Desta forma, sua função de distribuição acumulada é dada por

$$
F(y)= \begin{cases}0, & \text { se } y<0 \\ \delta_{0}+(1-\delta) \Phi\left(\frac{y-\mu}{\sigma}\right)^{\alpha}, & \text { se } 0 \leq y<1 \\ 1, & \text { se } y \geq 1\end{cases}
$$

A função de verossimilhança de uma observação da variável $Y$ correspondente ao modelo tobit normal inflacionado é dada por

$$
\begin{aligned}
f(y)= & \left\{\delta_{0}+(1-\delta) \Phi\left(\frac{-\mu}{\sigma}\right)^{\alpha}\right\}^{I_{0}}\left\{\delta_{1}+(1-\delta)\left[1-\Phi\left(\frac{1-\mu}{\sigma}\right)^{\alpha}\right]\right\}^{I_{1}} \\
& \times\left\{(1-\delta) \frac{\alpha}{\sigma} \phi\left(\frac{y-\mu}{\sigma}\right) \Phi\left(\frac{y-\mu}{\sigma}\right)^{\alpha-1}\right\}^{1-I_{0}-I_{1}}
\end{aligned}
$$


onde as variáveis auxiliares $I_{0}$ e $I_{1}$ são da mesma forma descrita anteriormente. Os momentos de interesse do modelo Tobit normal-potência duplamente censurado são difíceis de serem obtidos analiticamente e portanto faz-se necessário o uso de algum método de aproximação numérica.

A Figura (2.7) ilustra algumas possíveis formas que a distribuição normal-potência pode apresentar no intervalo $[0,1]$ para diferentes valores de $\alpha, \mu$ e $\sigma$.

Um exemplo das curvas das funções de distribuição acumulada e das funções de densidade das variáveis tobit normal-potência inflacionada de zeros e tobit normal-potência inflacionada de zeros e uns podem ser observadas nas Figuras (2.8) e (2.9).
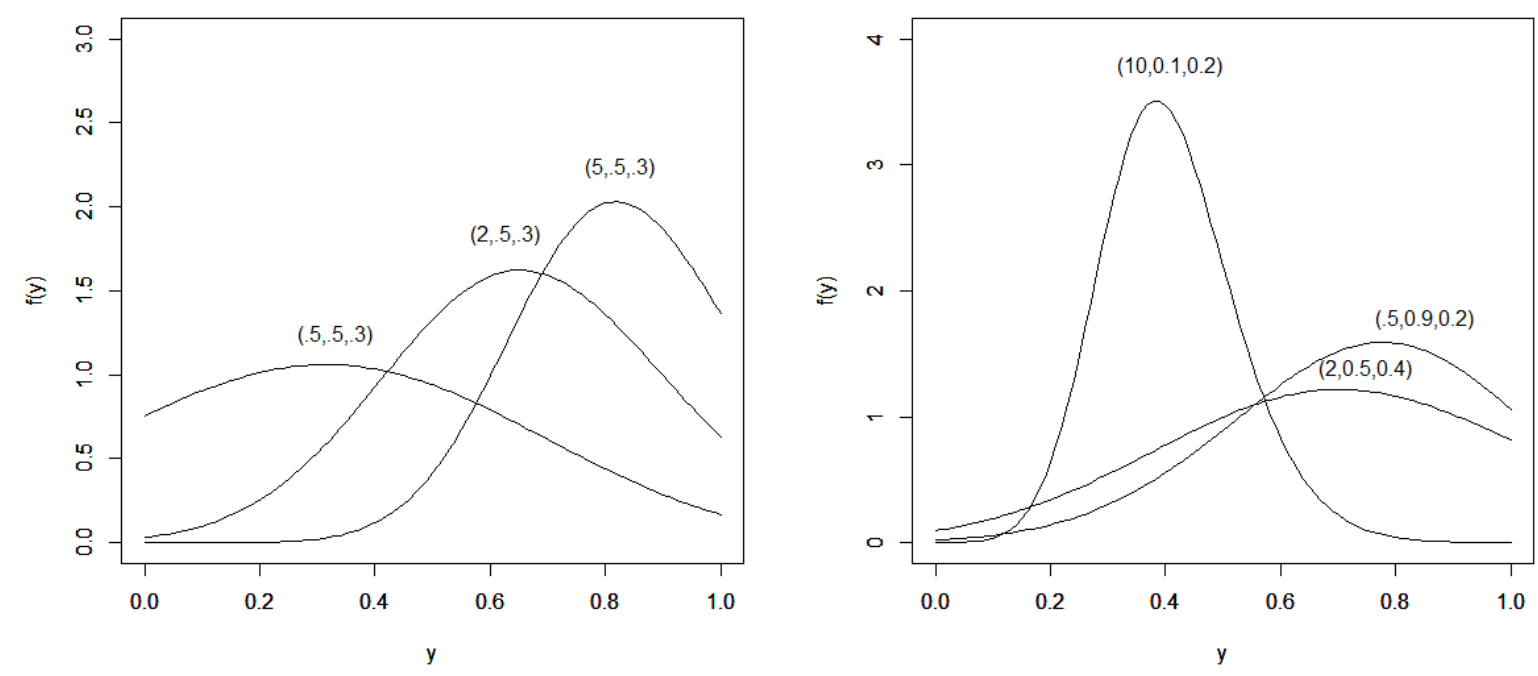

Figura 2.7: Gráfico das densidades Tobit normal-potência inflacionada para diferentes valores de $(\alpha, \mu, \sigma)$.
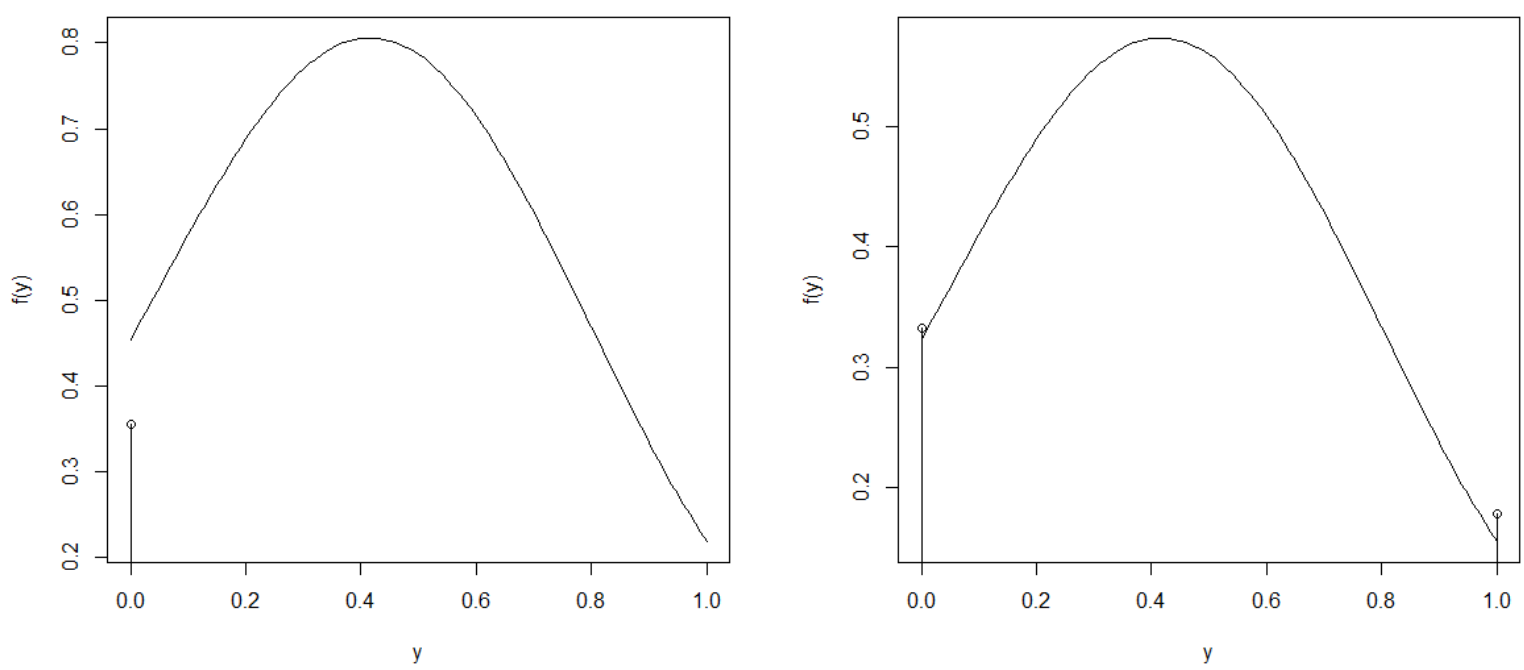

Figura 2.8: Gráfico das funções de distribuição acumulada das variáveis Tobit normal-potência inflacionada de zeros (esquerda) e Tobit normal-potência inflacionada de zeros e uns (direita), considerando os parâmetros $(\delta=0.24, \alpha=0.5, \mu=0.6, \sigma=0.3) e\left(\delta_{0}=0.24, \delta_{1}=0.15, \alpha=0.5, \mu=0.6, \sigma=0.3\right)$, respectivamente. 

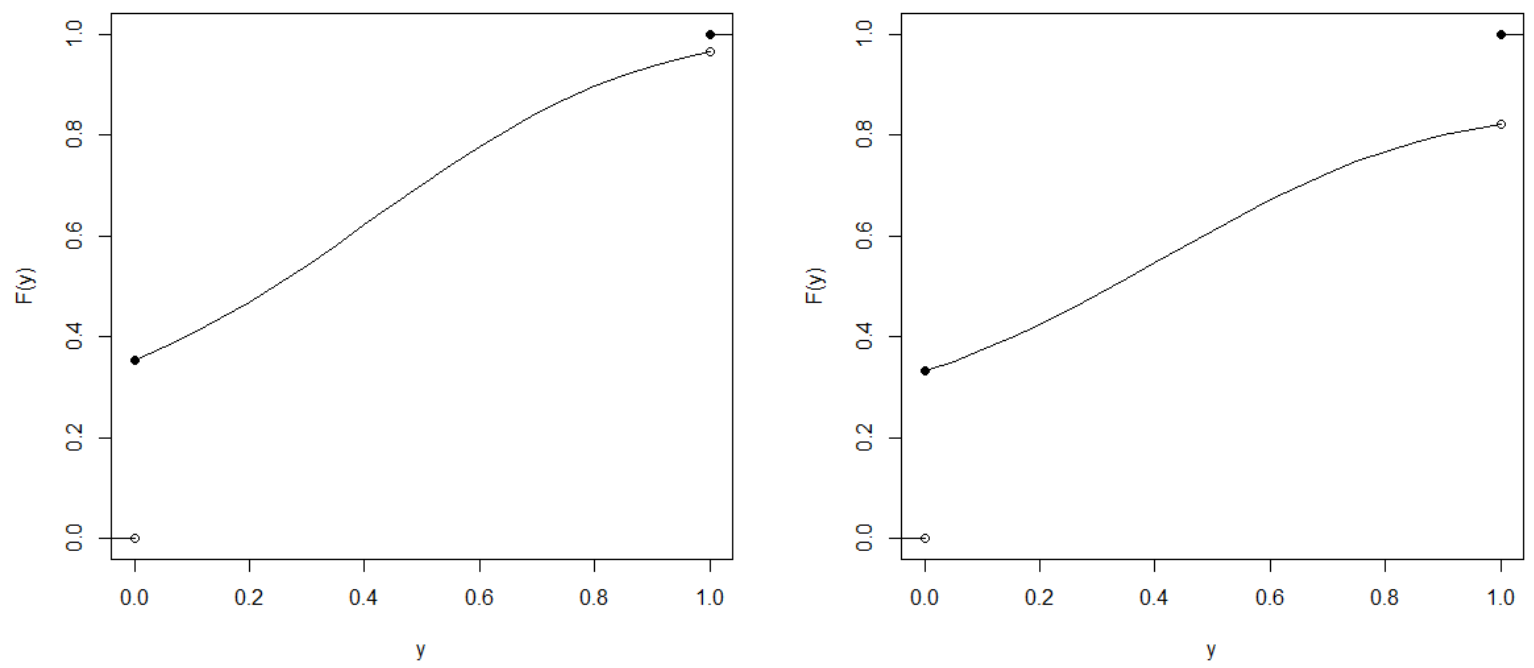

Figura 2.9: Gráfico das funções de verossimilhança das variáveis Tobit normal-potência inflacionada de zeros (esquerda) e Tobit normal-potência inflacionada de zeros e uns (direita), considerando os parâmetros $(\delta=0.24, \mu=0.6, \sigma=0.3) e\left(\delta_{0}=0.24, \delta_{1}=0.15, \alpha=0.5, \mu=0.6, \sigma=0.3\right)$, respectivamente. 


\section{Capítulo 3}

\section{Modelos de Regressão}

No contexto de modelos de regressão vamos inicialmente apresentar a versão Bayesina para o modelos Beta inflacionados e dos modelos tobit duplamente censurados. Na seção 3.1 vamos apresentar o modelo de regressão beta inflacionado, na subseção 3.2 apresentaremos a classe de modelos Tobit normal duplamente censurados e inflacionados e será mostrado o modelo Tobit normal-potência duplamente censurado e inflacionado na seção 3.3. Em seguida apresentaremos um estudo de simulação com o intuito de explorar a competitividade das duas classes de modelos. Por fim será apresentado uma aplicação dos modelos a conjuntos de dados reais. Este capítulo tem como contribuição o desenvolvimento da abordagem Bayesiana para estes modelos.

\subsection{Modelos de regressão beta inflacionado}

Para o modelo de regressão beta inflacionado de zero ou um $\left(B I_{c}\right)$ apresentado por Ospina (2008) considere $y_{1}, \ldots, y_{n}$ variáveis aleatórias cada uma com função de densidade beta inflacionada no ponto $c(c=0$ ou $c=1)$, isto é, $y_{i} \sim B I_{c}\left(\alpha_{i}, \mu_{i}, \phi\right)$. O modelo de regressão é composto por funções de ligação que estabelecem a relação entre os parâmetros da distribuição com um conjunto de covariáveis. Sendo assim, o modelo é definido pelo conjunto de equações da forma

$$
\begin{aligned}
& h\left(\alpha_{i}\right)=\boldsymbol{z}_{i}^{T} \boldsymbol{\gamma}=\sum_{j=1}^{m} z_{i j} \gamma_{j}=\varsigma_{i}, \\
& g\left(\mu_{i}\right)=\boldsymbol{x}_{i}^{T} \boldsymbol{\beta}=\sum_{j=1}^{p} x_{i j} \beta_{j}=\eta_{i},
\end{aligned}
$$

onde $\boldsymbol{\gamma}=\left(\gamma_{1}, \ldots, \gamma_{m}\right)^{T}$ e $\boldsymbol{\beta}=\left(\beta_{1}, \ldots, \beta_{m}\right)^{T}$ são os vetores de parâmetros desconhecidos, $z_{i j}, \ldots, z_{i m}$ e $x_{i j}, \ldots, x_{i p}$ são as variáveis explicativas conhecidas correspondentes a observação $i$, para $i=$ $1, \ldots, n$. É necessária a condição $m+p<n$ e as covariáveis $z$ 's e $x$ 's podem coincidir total ou parcialmente. É natural que sejam escolhidas funções de ligação do tipo $h:(0,1) \rightarrow \mathbb{R}$ e $g$ : $(0,1) \rightarrow \mathbb{R}$. Na literatura as funções de ligação mais utilizadas são a especificação logito $(g(\mu)=$ $\log \{\mu /(1-\mu)\})$, probito $\left(g(\mu)=\Phi^{-1}(\mu)\right)$, complemento $\log -\log (g(\mu)=\log \{-\log (1-\mu)\})$ e $\log -\log$ $(g(\mu)=\log \{-\log (\mu)\})$. As mesmas especificações podem ser utilizadas tanto para $\mu$ como para $\alpha$. Temos $\alpha_{i}=P\left(y_{i}=c\right), \mu_{i}=E\left(y_{i} \mid y_{i} \in(0,1)\right)$ e $\phi$ fixo para todas as observações. É possível aumentar a complexidade do modelo permitindo que o parâmetro de precisão $\phi$ varie entre as observações e este pode estar relacionado a uma função de ligação. Uma função de ligação, $t($.$) , bastante utilizada$ para parâmetros de precisão da forma $t: \mathbb{R}^{+} \rightarrow \mathbb{R}$ é a função logaritmica. A função é expressa da seguinte forma:

$$
t\left(\phi_{i}\right)=\boldsymbol{u}_{i}^{T} \boldsymbol{\omega}=\sum_{j=1}^{l} u_{i j} \omega_{j}=\psi_{i},
$$


onde $\boldsymbol{\omega}=\left(\omega_{1}, \ldots, \omega_{l}\right)^{T}$ é o vetor de parâmetros desconhecidos, $u_{i j}, \ldots, u_{i l}$ são as variáveis explicativas e $t($.$) pode ser especificada como \log ($.$) .$

Se considerarmos o vetor de parâmetros $\boldsymbol{\theta}=\left(\boldsymbol{\gamma}^{T}, \boldsymbol{\beta}^{T}, \phi\right)^{T}$ para o caso de $\phi$ fixo, a função de verossimilhança para o modelo de regressão $B I_{c}$ é da forma

$$
L(\theta)=\prod_{i=1}^{n} b i_{c}\left(y_{i} ; \alpha_{i}, \mu_{i}, \phi\right)=L_{1}(\gamma) L_{2}(\boldsymbol{\beta}, \phi)
$$

em que

$$
\begin{aligned}
L_{1}(\gamma) & =\prod_{i=1}^{n} \alpha_{i}^{I_{c i}}\left(1-\alpha_{i}\right)^{1-I_{c i}}, \\
L_{2}(\boldsymbol{\beta}, \phi) & =\prod_{i=1}^{n}\left\{f\left(y_{i}, \mu_{i}, \phi\right)\right\}^{1-I_{c i}},
\end{aligned}
$$

onde $f\left(y_{i}, \mu_{i}, \phi\right)$ é a função de densidade beta e $I_{c i}$ é a variável auxiliar correspondente a observação $i$ da forma

$$
I_{c i}= \begin{cases}1, & \text { se } y_{i}=c \\ 0, & \text { caso contrário. }\end{cases}
$$

Considerando o caso em que o parâmetro varia entre as observações, temos o vetor de parâmetros $\boldsymbol{\theta}=\left(\boldsymbol{\gamma}^{T}, \boldsymbol{\beta}^{T}, \boldsymbol{\omega}^{T}\right)^{T}$ e a função de verossimilhança é da forma:

$$
L(\theta)=\prod_{i=1}^{n} b i_{c}\left(y_{i} ; \alpha_{i}, \mu_{i}, \phi_{i}\right)=L_{1}(\gamma) L_{2}(\boldsymbol{\beta}, \boldsymbol{\omega}),
$$

em que

$$
\begin{aligned}
L_{1}(\gamma) & =\prod_{i=1}^{n} \alpha_{i}^{I_{c i}}\left(1-\alpha_{i}\right)^{1-I_{c i}}, \\
L_{2}(\boldsymbol{\beta}, \boldsymbol{\omega}) & =\prod_{i=1}^{n}\left\{f\left(y_{i}, \mu_{i}, \phi_{i}\right)\right\}^{1-I_{c i}},
\end{aligned}
$$

Para o modelo de regressão beta inflacionado de zero e um $(B I Z U)$ apresentado por Ospina (2008) considere $y_{1}, \ldots, y_{n}$ variáveis aleatórias cada uma com função de densidade beta inflacionada de zero e um, isto é, $y_{i} \sim B I Z U\left(\delta_{0 i}, \delta_{1 i}, \mu_{i}, \phi\right)$. O modelo de regressão é composto por funções de ligação que estabelecem a relação entre os parâmetros da distribuição com um conjunto de covariáveis. Sendo assim, o modelo é definido pelo conjunto de equações da forma

$$
\begin{aligned}
\boldsymbol{H}\left(\delta_{0 i}, \delta_{1 i}\right) & =\left(h_{0}\left(\delta_{0 i}, \delta_{1 i}\right), h_{1}\left(\delta_{0 i}, \delta_{1 i}\right)\right)=\left(\varsigma_{0 i}, \varsigma_{1 i}\right), \\
g\left(\mu_{i}\right) & =\boldsymbol{x}_{i}^{T} \boldsymbol{\beta}=\sum_{j=1}^{p} x_{i j} \beta_{j}=\eta_{i},
\end{aligned}
$$

onde temos as preditoras lineares $\eta_{i}=\boldsymbol{x}_{i}^{T} \boldsymbol{\beta}, \varsigma_{0 i}=\boldsymbol{v}_{\boldsymbol{i}}^{T} \boldsymbol{\rho}$ e $\varsigma_{1 i}=\boldsymbol{z}_{\boldsymbol{i}}^{T} \boldsymbol{\gamma}, \operatorname{com} \boldsymbol{\beta}=\left(\beta_{1}, \ldots, \beta_{m}\right)^{T}$, $\boldsymbol{\rho}=\left(\rho_{1}, \ldots, \rho_{m_{0}}\right)^{T}$ e $\boldsymbol{\gamma}=\left(\gamma_{1}, \ldots, \gamma_{m_{1}}\right)^{T}$ os vetores de parâmetros desconhecidos tais que $\boldsymbol{\beta} \in$ $\mathbb{R}^{p}, \boldsymbol{\rho} \in \mathbb{R}^{m_{0}}$ e $\boldsymbol{\gamma} \in \mathbb{R}^{m_{1}}$. Os vetores $\boldsymbol{v}_{i}=\left(v_{1 j}, \ldots, v_{i m_{0}}\right)^{T}, \boldsymbol{z}_{i}=\left(z_{i 1}, \ldots, z_{i m_{1}}\right)^{T}$ e $\boldsymbol{x}_{i}=\left(x_{i 1}, \ldots, x_{i p}\right)^{T}$ representam as variáveis explicativas conhecidas correspondentes a observação $i$, para $i=1, \ldots, n$. Temos $\mu_{i}=E\left(y_{i} \mid y_{i} \in(0,1)\right), \delta_{0 i}=P\left(y_{i}=0\right), \delta_{1 i}=P\left(y_{i}=1\right), 1-\delta_{0 i}-\delta_{1 i}=P\left(y_{i} \in(0,1)\right) \mathrm{e}$ estamos considerando $\phi$ fixo para todas as observações.

A função $\boldsymbol{H}$ deve ser uma transformação bijetora do conjunto $\mathcal{C}=\left\{\left(\delta_{0 i}, \delta_{1 i}\right): 0<\delta_{0 i}<\right.$ $\left.1,0<\delta_{1 i}<1-\delta_{0 i}\right\}$ a $\mathbb{R}^{2}$, duplamente diferenciável. Sob estas condições, as derivadas parciais de $\delta_{0 i}=h_{0}^{*}\left(\varsigma_{0 i}, \varsigma_{1 i}\right)$ e de $\delta_{1 i}=h_{1}^{*}\left(\varsigma_{0 i}, \varsigma_{1 i}\right)$ são contínuas em $\mathbb{R}^{2}$ e $\delta_{0 i}, \delta_{1 i}$ podem ser escritos em termos 
de $\varsigma_{0 i}, \varsigma_{1 i}$ de forma única (Rudin, 1976, Teo 9.28, p.224). Uma opção razoável é definir $\boldsymbol{H}$ da forma

$$
\boldsymbol{H}\left(\delta_{0 i}, \delta_{0 i}\right)=\left(h_{0}\left(\delta_{0 i}, \delta_{1 i}\right), h_{1}\left(\delta_{0 i}, \delta_{1 i}\right)\right)=\left(h\left(\frac{\delta_{0 i}}{1-\delta_{0 i}-\delta_{1 i}}\right), h\left(\frac{\delta_{1 i}}{1-\delta_{0 i}-\delta_{1 i}}\right)\right),
$$

$\operatorname{com} h: \mathbb{R}^{+} \rightarrow \mathbb{R}$ estritamente monótona e duas vezes diferenciável.

Seguindo o mesmo padrão de escolha das funções de ligação do modelo de regressão $B I_{c}$, podemos escolher $h$ como a função logaritmo. Assim, temos $h_{0}\left(\delta_{0 i}, \delta_{1 i}\right)=\log \left(\delta_{0 i} /\left(1-\delta_{0 i}-\delta_{1 i}\right)\right)=\varsigma_{0 i}$ e $h_{1}\left(\delta_{1 i}, \delta_{1 i}\right)=\log \left(\delta_{0 i} /\left(1-\delta_{0 i}-\delta_{1 i}\right)\right)=\varsigma_{1 i}$ e portanto,

$$
\begin{aligned}
& \frac{P\left(Y_{i}=0\right)}{P\left(Y_{i} \in(0,1)\right)}=\frac{\delta_{0 i}}{1-\delta_{0 i}-\delta_{1 i}}=\exp \left(\varsigma_{0 i}\right), \\
& \frac{P\left(Y_{i}=1\right)}{P\left(Y_{i} \in(0,1)\right)}=\frac{\delta_{1 i}}{1-\delta_{0 i}-\delta_{1 i}}=\exp \left(\varsigma_{1 i}\right),
\end{aligned}
$$

isto é,

$$
\begin{aligned}
\delta_{0 i} & =P\left(Y_{i}=0\right)=\frac{e^{\varsigma_{0 i}}}{1+e^{\varsigma_{0 i}}+e^{\varsigma_{1 i}}}, \\
\delta_{1 i} & =P\left(Y_{i}=1\right)=\frac{e^{\varsigma_{1 i}}}{1+e^{\varsigma_{0 i}}+e^{\varsigma_{1 i}}}, \\
1-\delta_{0 i}-\delta_{1 i} & =P\left(Y_{i} \in(0,1)\right)=\frac{1}{1+e^{\varsigma_{0 i}}+e^{\varsigma_{1 i}}} .
\end{aligned}
$$

É possível aumentar a complexidade do modelo permitindo que o parâmetro de precisão $\phi$ varie entre as observações e este pode ser ligado a uma função de ligação. Pode ser utilizada uma função de ligação para $\phi_{i}$ da forma:

$$
t\left(\xi_{i}\right)=\boldsymbol{u}_{i}^{T} \boldsymbol{\omega}=\sum_{j=1}^{l} u_{i j} \omega_{j}=\psi_{i},
$$

onde $\boldsymbol{\omega}=\left(\omega_{1}, \ldots, \omega_{l}\right)^{T}$ é o vetor de parâmetros desconhecidos, $u_{i j}, \ldots, u_{i l}$ são as variáveis explicativas e $t($.$) pode ser especificada como \log ($.$) .$

Se considerarmos o vetor de parâmetros $\boldsymbol{\theta}=\left(\boldsymbol{\rho}^{T}, \boldsymbol{\gamma}^{T}, \boldsymbol{\beta}^{T}, \phi\right)^{T}$, a função de verossimilhança para o modelo de regressão $B I Z U$ é da forma

$$
L(\theta)=\prod_{i=1}^{n} b i z u\left(y_{i} ; \delta_{0 i}, \delta_{1 i}, \mu_{i}, \phi\right)=L_{1}(\boldsymbol{\rho}, \boldsymbol{\gamma}) L_{2}(\boldsymbol{\beta}, \phi),
$$

em que

$$
\begin{aligned}
& L_{1}(\boldsymbol{\rho}, \boldsymbol{\gamma})=\prod_{i=1}^{n} \delta_{0 i}^{I_{0 i}} \delta_{1 i}^{I_{1 i}}\left(1-\delta_{0 i}-\delta_{1 i}\right)^{1-I_{0 i}-I_{1 i}}, \\
& L_{2}(\boldsymbol{\beta}, \phi)=\prod_{i=1}^{n}\left\{f\left(y_{i}, \mu_{i}, \phi\right)\right\}^{1-I_{0 i}-I_{1 i}},
\end{aligned}
$$

onde $f\left(y_{i}, \mu_{i}, \phi\right)$ é a função de densidade beta, $I_{0 i}$ e $I_{1 i}$ são as variáveis auxiliar correspondente a observação $i$ da forma

$$
I_{0 i}= \begin{cases}1, & \text { se } y_{i}=0, \\ 0, & \text { caso contrário }\end{cases}
$$


$\mathrm{e}$

$$
I_{1 i}= \begin{cases}1, & \text { se } y_{i}=1, \\ 0, & \text { caso contrário, }\end{cases}
$$

Considerando o caso em que o parâmetro de precisão está variando entre as observações, temos o vetor de parâmetros $\boldsymbol{\theta}=\left(\boldsymbol{\rho}^{T}, \boldsymbol{\gamma}^{T}, \boldsymbol{\beta}^{T}, \boldsymbol{\omega}^{T}\right)^{T}$ e a função de verossimilhança é da forma:

$$
L(\theta)=\prod_{i=1}^{n} b i z u\left(y_{i} ; \delta_{0 i}, \delta_{1 i}, \mu_{i}, \phi\right)=L_{1}(\boldsymbol{\rho}, \boldsymbol{\gamma}) L_{2}(\boldsymbol{\beta}, \boldsymbol{\omega}),
$$

em que

$$
\begin{aligned}
L_{1}(\boldsymbol{\rho}, \boldsymbol{\gamma}) & =\prod_{i=1}^{n} \delta_{0 i}^{I_{0 i}} \delta_{1 i}^{I_{1 i}}\left(1-\delta_{0 i}-\delta_{1 i}\right)^{1-I_{0 i}-I_{1 i}} \\
L_{2}(\boldsymbol{\beta}, \boldsymbol{\omega}) & =\prod_{i=1}^{n}\left\{f\left(y_{i}, \mu_{i}, \phi_{i}\right)\right\}^{1-I_{0 i}-I_{1 i}}
\end{aligned}
$$

É importante notar que a função de verossimilhança $L(\boldsymbol{\theta})$ dos modelos de regressão $B I_{c}$ e $B I Z U$ pode ser fatorada em dois termos, onde um depente apenas do vetor de parâmetros correspondente a componente discreta do modelo e o outro que depende somente do vetor de parâmetros correspondente a componente contínua do modelo e portanto estes dois conjuntos de parâmetros são independentes a inferência Bayesiana sobre um conjunto pode ser feita de forma independente do outro.

\subsection{Modelos de regressão Tobit normal duplamente censurado e inflacionado}

Para o modelo de regressão Tobit normal inflacionado de zero e um utilizamos uma adaptação do modelo proposto por Moulton e Halsey (1995). Considere $y_{1}, \ldots, y_{n}$ variáveis aleatórias cada uma com função de densidade Tobit normal inflacionada de zero e um, isto é, com probabilidade $\delta_{0 i}, y_{i}=0$, com probabilidade $\delta_{1 i}, y_{i}=1$ e considerando $\delta_{i}=\delta_{0 i}+\delta_{1 i}$, temos que com probabilidade $1-\delta_{i}, y_{i}$ segue o modelo Tobit duplamente censurado da forma:

$$
y_{i}= \begin{cases}0 & \text { se } y_{i}^{*} \leq 0 \\ y_{i}^{*} & \text { se } 0<w<1 \\ 1 & \text { se } y_{i}^{*} \geq 1\end{cases}
$$

onde $y_{i}^{*}$ corresponde a variável latente do modelo Tobit e no caso Tobit normal temos $y_{i}^{*} \sim N\left(\mu_{i}, \sigma^{2}\right)$, para $i=1, \ldots, n$. Assim como o modelo de regressão $B I Z U$, o modelo de regressão Tobit duplamente censurado e inflacionado é composto por funções de ligação que estabelecem a relação entre os parâmetros da distribuição com um conjunto de covariáveis. Sendo assim, o modelo é definido pelo conjunto de equações da forma

$$
\begin{aligned}
\boldsymbol{H}\left(\delta_{0 i}, \delta_{1 i}\right) & =\left(h_{0}\left(\delta_{0 i}, \delta_{1 i}\right), h_{1}\left(\delta_{0 i}, \delta_{1 i}\right)\right)=\left(\varsigma_{0 i}, \varsigma_{1 i}\right), \\
g\left(\mu_{i}\right) & =\boldsymbol{x}_{i}^{T} \boldsymbol{\beta}=\sum_{j=1}^{p} x_{i j} \beta_{j}=\eta_{i},
\end{aligned}
$$

onde temos as preditoras lineares $\eta_{i}=\boldsymbol{x}_{i}^{T} \boldsymbol{\beta}, \varsigma_{0 i}=\boldsymbol{v}_{\boldsymbol{i}}^{T} \boldsymbol{\rho}$ e $\varsigma_{1 i}=\boldsymbol{z}_{\boldsymbol{i}}^{T} \boldsymbol{\gamma}, \operatorname{com} \boldsymbol{\beta}=\left(\beta_{1}, \ldots, \beta_{m}\right)^{T}$, $\boldsymbol{\rho}=\left(\rho_{1}, \ldots, \rho_{m_{0}}\right)^{T}$ e $\boldsymbol{\gamma}=\left(\gamma_{1}, \ldots, \gamma_{m_{1}}\right)^{T}$ os vetores de parâmetros desconhecidos tais que $\boldsymbol{\beta} \in$ $\mathbb{R}^{p}, \boldsymbol{\rho} \in \mathbb{R}^{m_{0}}$ e $\boldsymbol{\gamma} \in \mathbb{R}^{m_{1}}$. Os vetores $\boldsymbol{v}_{i}=\left(v_{1 j}, \ldots, v_{i m_{0}}\right)^{T}, \boldsymbol{z}_{i}=\left(z_{i 1}, \ldots, z_{i m_{1}}\right)^{T}$ e $\boldsymbol{x}_{i}=\left(x_{i 1}, \ldots, x_{i p}\right)^{T}$ representam as variáveis explicativas conhecidas correspondentes a observação $i$, para $i=1, \ldots, n$. 
Temos $\mu_{i}=E\left(y_{i}^{*}\right), \delta_{0 i}$ corresponde a probabilidade de $y_{i}=0$ proveniente da componente de inflação, $\delta_{1 i}$ corresponde a probabilidade de $y_{i}=1$ proveniente da componente de inflação e $1-\delta_{0 i}-\delta_{1 i}$ corresponde a probabilidade de $y_{i}$ vir do modelo tobit duplamente censurado. Desta forma, temos

$$
\begin{aligned}
P\left(Y_{i}=0\right) & =\delta_{0 i}+\left(1-\delta_{i}\right) \Phi\left(\frac{-\mu_{i}}{\sigma}\right), \\
P\left(Y_{i}=1\right) & =\delta_{1 i}+\left(1-\delta_{i}\right)\left[1-\Phi\left(\frac{1-\mu_{i}}{\sigma}\right)\right], \\
\mathrm{e} & \\
P\left(Y_{i} \in(0,1)\right) & =\left(1-\delta_{i}\right)\left[\Phi\left(\frac{-\mu_{i}}{\sigma}\right)-\Phi\left(\frac{1-\mu_{i}}{\sigma}\right)\right] .
\end{aligned}
$$

Note que estamos considerando $\sigma$ fixo para todas as observações. Apesar da distribuição assumir $\mu_{i} i n \mathbb{R}$, é razoável restringirmos no modelo $\mu_{i} \in(0,1)$, para $i=1, \ldots, n$, uma vez que a componente contínua da variável resposta está restrita ao intervalo $(0,1)$. Portanto, a função $g$ pode ser escolhida de forma que $g:(0,1) \rightarrow \mathbb{R}$. Assim como no modelo de regressão $B I Z U, \boldsymbol{H}$ deve ser uma transformação bijetora do conjunto $\mathcal{C}=\left\{\left(\delta_{0 i}, \delta_{1 i}\right): 0<\delta_{0 i}<1,0<\delta_{1 i}<1-\delta_{0 i}\right\}$ a $\mathbb{R}^{2}$, duplamente diferenciável. Seguindo o mesmo padrão de escolha das funções de ligação do modelo de regressão $B I Z U$, podemos escolher $h$ como a função logaritmo. Assim, temos $h_{0}\left(\delta_{0 i}, \delta_{1 i}\right)=\log \left(\delta_{0 i} /\left(1-\delta_{0 i}-\delta_{1 i}\right)\right)=\varsigma_{0 i}$ e $h_{1}\left(\delta_{1 i}, \delta_{1 i}\right)=\log \left(\delta_{0 i} /\left(1-\delta_{0 i}-\delta_{1 i}\right)\right)=\varsigma_{1 i}$.

É possível aumentar a complexidade do modelo admitindo heterocedasticidade, permitindo que $\sigma$ varie entre as observações ligado a uma função de ligação. Desta forma, temos

$$
t\left(\sigma_{i}\right)=\boldsymbol{u}_{i}^{T} \boldsymbol{\omega}=\sum_{j=1}^{l} u_{i j} \omega_{j}=\psi_{i},
$$

onde $\boldsymbol{\omega}=\left(\omega_{1}, \ldots, \omega_{l}\right)^{T}$ é o vetor de parâmetros desconhecidos, $u_{i j}, \ldots, u_{i l}$ são as variáveis explicativas e $t($.$) pode ser especificada da forma t: \mathbb{R}^{+} \rightarrow \mathbb{R}$. Nos modelos normais heterocedásticos costuma-se escolher $t$ como a função logaritmo.

Se considerarmos o vetor de parâmetros $\boldsymbol{\theta}=\left(\boldsymbol{\rho}^{T}, \boldsymbol{\gamma}^{T}, \boldsymbol{\beta}^{T}, \sigma\right)^{T}$ para o caso de $\sigma$ fixo, a função de verossimilhança para o modelo de regressão tobit duplamente censurado é da forma

$$
\begin{aligned}
L(\theta)= & \prod_{i=1}^{n}\left\{\delta_{0 i}+\left(1-\delta_{0 i}-\delta_{1 i}\right) \Phi\left(\frac{-\mu_{i}}{\sigma}\right)\right\}^{I_{0 i}} \\
& \times\left\{\delta_{1 i}+\left(1-\delta_{0 i}-\delta_{1 i}\right)\left[1-\Phi\left(\frac{1-\mu_{i}}{\sigma}\right)\right]\right\}^{I_{1 i}} \\
& \times\left\{\left(1-\delta_{0 i}-\delta_{1 i}\right)\left[\frac{1}{\sigma} \phi\left(\frac{y_{i}-\mu_{i}}{\sigma}\right)\right]\right\}^{1-I_{0 i}-I_{1 i}}
\end{aligned}
$$

onde $\Phi($.$) e \phi($.$) correspondem a função de distribuição acumulada e função de densidade da distri-$ buição normal padrão, $I_{0 i}$ e $I_{1 i}$ são as variáveis auxiliar correspondente a observação $i$ da forma

$$
I_{0 i}= \begin{cases}1, & \text { se } y_{i}=0, \\ 0, & \text { caso contrário }\end{cases}
$$

e

$$
I_{1 i}= \begin{cases}1, & \text { se } y_{i}=1, \\ 0, & \text { caso contrário, }\end{cases}
$$

Considerando o caso em que temos $\sigma$ variando entre as observações, temos o vetor de parâmetros 
$\boldsymbol{\theta}=\left(\boldsymbol{\rho}^{T}, \boldsymbol{\gamma}^{T}, \boldsymbol{\beta}^{T}, \boldsymbol{\omega}^{T}\right)^{T}$ e a função de verossimilhança é da forma:

$$
\begin{aligned}
L(\theta)= & \prod_{i=1}^{n}\left\{\delta_{0 i}+\left(1-\delta_{0 i}-\delta_{1 i}\right) \Phi\left(\frac{-\mu_{i}}{\sigma_{i}}\right)\right\}^{I_{0 i}} \\
& \times\left\{\delta_{1 i}+\left(1-\delta_{0 i}-\delta_{1 i}\right)\left[1-\Phi\left(\frac{1-\mu_{i}}{\sigma_{i}}\right)\right]\right\}^{I_{1 i}} \\
& \times\left\{\left(1-\delta_{0 i}-\delta_{1 i}\right)\left[\frac{1}{\sigma} \phi\left(\frac{y_{i}-\mu_{i}}{\sigma_{i}}\right)\right]\right\}^{1-I_{0 i}-I_{1 i}} .
\end{aligned}
$$

Os modelos mais simples podem ser obtidos de forma direta. Para o Tobit normal duplamente censurado e inflacionado de zero, basta tomar $\delta_{1 i}=0$, para $i=1, \ldots, n$ e utilizar $h_{0}=h$ do tipo $h:(0,1) \rightarrow \mathbb{R}$. Para o Tobit normal duplamente censurado e inflacionado de um, tome $\delta_{0 i}=0$, para $i=1, \ldots, n$ e $h_{1}=h$ do tipo $h:(0,1) \rightarrow \mathbb{R}$. Podemos ainda utilizar o modelo Tobit normal duplamente censurado sem inflação, tomando $\delta_{0 i}=\delta_{1 i}=0$, para $i=1, \ldots, n$.

É possível ainda a adaptação ao modelo proposto por Cragg (1971), onde não há censura e a massa pontual dos dados vem somente da componente de inflação, ou seja

$$
\begin{aligned}
P\left(Y_{i}=0\right) & =\delta_{0 i}, \\
P\left(Y_{i}=1\right) & =\delta_{1 i}, \\
\mathrm{e} & \\
P\left(Y_{i} \in(0,1)\right) & =1-\delta_{0 i}-\delta_{1 i}=1-\delta_{i} .
\end{aligned}
$$

Se considerarmos o vetor de parâmetros $\boldsymbol{\theta}=\left(\boldsymbol{\rho}^{T}, \boldsymbol{\gamma}^{T}, \boldsymbol{\beta}^{T}, \sigma\right)^{T}$ para o caso de $\sigma$ fixo, a função de verossimilhança para o modelo de regressão tobit duplamente censurado é da forma

$$
L(\theta)=L_{1}(\boldsymbol{\rho}, \gamma) L_{2}(\boldsymbol{\beta}, \sigma)
$$

em que

$$
\begin{aligned}
& L_{1}(\boldsymbol{\rho}, \gamma)=\prod_{i=1}^{n} \delta_{0 i}^{I_{0 i}} \delta_{1 i}^{I_{1 i}}\left(1-\delta_{0 i}-\delta_{1 i}\right)^{1-I_{0 i}-I_{1 i},} \\
& L_{2}(\boldsymbol{\beta}, \sigma)=\prod_{i=1}^{n}\left\{\frac{\frac{1}{\sigma} \phi\left(\frac{y_{i}-\mu_{i}}{\sigma_{i}}\right)}{\Phi\left(\frac{1-\mu_{i}}{\sigma}\right)-\Phi\left(\frac{-\mu_{i}}{\sigma}\right)}\right\}^{1-I_{0 i}-I_{1 i}} .
\end{aligned}
$$

Note que assim como nos modelos de regressão $B I_{c}$ e $B I Z U$, a função de verossimilhança $L(\boldsymbol{\theta})$ pode ser fatorada em dois termos, onde um depende apenas do vetor de parâmetros correspondente a componente discreta do modelo e o outro que depende somente do vetor de parâmetros correspondente a componente contínua do modelo e portanto estes dois conjuntos de parâmetros são independentes a inferência Bayesiana sobre um conjunto pode ser feita de forma independente do outro.

\subsection{Modelos de regressão Tobit normal-potência duplamente cen- surado e inflacionado}

Para o modelo de regressão Tobit normal-potência inflacionado de zero e um seguiremos a mesma abordagem do modelo Tobit normal duplamente censurado e inflacionado com a diferença que a variável latente segue uma distribuição $N P\left(\alpha, \mu_{i}, \sigma^{2}\right)$, com densidade definida pela equação (2.4). Desta forma, considere $y_{1}, \ldots, y_{n}$ variáveis aleatórias cada uma com função de densidade Tobit normal-potência inflacionada de zero e um, isto é, com probabilidade $\delta_{0 i}, y_{i}=0$, com probabilidade $\delta_{1 i}, y_{i}=1$ e considerando $\delta_{i}=\delta_{0 i}+\delta_{1 i}$, temos que com probabilidade $1-\delta_{i}, y_{i}$ segue o modelo 
Tobit normal-potência duplamente censurado da forma:

$$
y_{i}= \begin{cases}0 & \text { se } y_{i}^{*} \leq 0 \\ y_{i}^{*} & \text { se } 0<w<1 \\ 1 & \text { se } y_{i}^{*} \geq 1\end{cases}
$$

onde $y_{i}^{*}$ corresponde a variável latente do modelo tobit, neste caso $y_{i}^{*} \sim N P\left(\alpha, \mu_{i}, \sigma^{2}\right)$, para $i=$ $1, \ldots, n$. Assim como o modelo de regressão tobit normal duplamente censurado e inflacionado, o modelo normal-potência é composto por funções de ligação que estabelecem a relação entre os parâmetros da distribuição com um conjunto de covariáveis. Sendo assim, o modelo é definido pelo conjunto de equações da forma

$$
\begin{aligned}
\boldsymbol{H}\left(\delta_{0 i}, \delta_{1 i}\right) & =\left(h_{0}\left(\delta_{0 i}, \delta_{1 i}\right), h_{1}\left(\delta_{0 i}, \delta_{1 i}\right)\right)=\left(\varsigma_{0 i}, \varsigma_{1 i}\right), \\
g\left(\mu_{i}\right) & =\boldsymbol{x}_{i}^{T} \boldsymbol{\beta}=\sum_{j=1}^{p} x_{i j} \beta_{j}=\eta_{i},
\end{aligned}
$$

onde temos as preditoras lineares $\eta_{i}=\boldsymbol{x}_{i}^{T} \boldsymbol{\beta}, \varsigma_{0 i}=\boldsymbol{v}_{\boldsymbol{i}}^{T} \boldsymbol{\rho}$ e $\varsigma_{1 i}=\boldsymbol{z}_{\boldsymbol{i}}{ }^{T} \boldsymbol{\gamma}, \operatorname{com} \boldsymbol{\beta}=\left(\beta_{1}, \ldots, \beta_{m}\right)^{T}$, $\boldsymbol{\rho}=\left(\rho_{1}, \ldots, \rho_{m_{0}}\right)^{T}$ e $\boldsymbol{\gamma}=\left(\gamma_{1}, \ldots, \gamma_{m_{1}}\right)^{T}$ os vetores de parâmetros desconhecidos tais que $\boldsymbol{\beta} \in$ $\mathbb{R}^{p}, \boldsymbol{\rho} \in \mathbb{R}^{m_{0}}$ e $\boldsymbol{\gamma} \in \mathbb{R}^{m_{1}}$. Os vetores $\boldsymbol{v}_{i}=\left(v_{1 j}, \ldots, v_{i m_{0}}\right)^{T}, \boldsymbol{z}_{i}=\left(z_{i 1}, \ldots, z_{i m_{1}}\right)^{T}$ e $\boldsymbol{x}_{i}=\left(x_{i 1}, \ldots, x_{i p}\right)^{T}$ representam as variáveis explicativas conhecidas correspondentes a observação $i$, para $i=1, \ldots, n$. Temos, portanto,

$$
\begin{aligned}
P\left(Y_{i}=0\right) & =\delta_{0 i}+\left(1-\delta_{i}\right) \Phi\left(\frac{-\mu_{i}}{\sigma}\right)^{\alpha}, \\
P\left(Y_{i}=1\right) & =\delta_{1 i}+\left(1-\delta_{i}\right)\left[1-\Phi\left(\frac{1-\mu_{i}}{\sigma}\right)^{\alpha}\right], \\
\mathrm{e} & \\
P\left(Y_{i} \in(0,1)\right) & =\left(1-\delta_{i}\right)\left[\Phi\left(\frac{-\mu_{i}}{\sigma}\right)^{\alpha}-\Phi\left(\frac{1-\mu_{i}}{\sigma}\right)^{\alpha}\right] .
\end{aligned}
$$

Note que estamos considerando $\sigma$ fixo para todas as observações. Apesar da distribuição assumir $\mu_{i} i n \mathbb{R}$, é razoável restringirmos no modelo $\mu_{i} \in(0,1)$, para $i=1, \ldots, n$, uma vez que a componente contínua da variável resposta está restrita ao intervalo $(0,1)$. Portanto a função $g$ pode ser escolhida de forma que $g:(0,1) \rightarrow \mathbb{R}$. Assim como no modelo de regressão $B I Z U, \boldsymbol{H}$ deve ser uma transformação bijetora do conjunto $\mathcal{C}=\left\{\left(\delta_{0 i}, \delta_{1 i}\right): 0<\delta_{0 i}<1,0<\delta_{1 i}<1-\delta_{0 i}\right\}$ a $\mathbb{R}^{2}$, duplamente diferenciável. Seguindo o mesmo padrão de escolha das funções de ligação do modelo de regressão apresentados, podemos escolher $h$ como a função logaritmo. Assim, temos $h_{0}\left(\delta_{0 i}, \delta_{1 i}\right)=\log \left(\delta_{0 i} /\left(1-\delta_{0 i}-\delta_{1 i}\right)\right)=\varsigma_{0 i}$ e $h_{1}\left(\delta_{1 i}, \delta_{1 i}\right)=\log \left(\delta_{0 i} /\left(1-\delta_{0 i}-\delta_{1 i}\right)\right)=\varsigma_{1 i}$.

É possível aumentar a complexidade do modelo admitindo heterocedasticidade, permitindo que $\sigma$ varie entre as observações ligado a uma função de ligação. Desta forma, temos

$$
t\left(\sigma_{i}\right)=\boldsymbol{u}_{i}^{T} \boldsymbol{\omega}=\sum_{j=1}^{l} u_{i j} \omega_{j}=\psi_{i},
$$

onde $\boldsymbol{\omega}=\left(\omega_{1}, \ldots, \omega_{l}\right)^{T}$ é o vetor de parâmetros desconhecidos, $u_{i j}, \ldots, u_{i l}$ são as variáveis explicativas e $t($.$) pode ser especificada da forma t: \mathbb{R}^{+} \rightarrow \mathbb{R}$.

Se considerarmos o vetor de parâmetros $\boldsymbol{\theta}=\left(\alpha, \boldsymbol{\rho}^{T}, \boldsymbol{\gamma}^{T}, \boldsymbol{\beta}^{T}, \sigma\right)^{T}$ para o caso de $\sigma$ fixo, a função 
de verossimilhança para o modelo de regressão tobit duplamente censurado é da forma

$$
\begin{aligned}
L(\theta)= & \prod_{i=1}^{n}\left\{\delta_{0 i}+\left(1-\delta_{0 i}-\delta_{1 i}\right) \Phi\left(\frac{-\mu_{i}}{\sigma}\right)^{\alpha}\right\}^{I_{0 i}} \\
& \times\left\{\delta_{1 i}+\left(1-\delta_{0 i}-\delta_{1 i}\right)\left[1-\Phi\left(\frac{1-\mu_{i}}{\sigma}\right)^{\alpha}\right]\right\}^{I_{1 i}} \\
& \times\left\{\left(1-\delta_{0 i}-\delta_{1 i}\right)\left[\frac{\alpha}{\sigma} \phi\left(\frac{y_{i}-\mu_{i}}{\sigma}\right) \Phi\left(\frac{y_{i}-\mu_{i}}{\sigma}\right)^{\alpha-1}\right]\right\}^{1-I_{0 i}-I_{1 i}},
\end{aligned}
$$

onde $\Phi($.$) e \phi($.$) correspondem a função de distribuição acumulada e função de densidade da distri-$ buição normal padrão, $I_{0 i}$ e $I_{1 i}$ são as variáveis auxiliar correspondente a observação $i$ da forma

$$
I_{0 i}= \begin{cases}1, & \text { se } y_{i}=0, \\ 0, & \text { caso contrário }\end{cases}
$$

$\mathrm{e}$

$$
I_{1 i}= \begin{cases}1, & \text { se } y_{i}=1, \\ 0, & \text { caso contrário, }\end{cases}
$$

Considerando o caso em que temos $\sigma$ variando entre as observações, temos o vetor de parâmetros $\boldsymbol{\theta}=\left(\alpha, \boldsymbol{\rho}^{T}, \boldsymbol{\gamma}^{T}, \boldsymbol{\beta}^{T}, \boldsymbol{\omega}^{T}\right)^{T}$ e a função de verossimilhança é da forma:

$$
\begin{aligned}
L(\theta)= & \prod_{i=1}^{n}\left\{\delta_{0 i}+\left(1-\delta_{0 i}-\delta_{1 i}\right) \Phi\left(\frac{-\mu_{i}}{\sigma_{i}}\right)^{\alpha}\right\}^{I_{0 i}} \\
& \times\left\{\delta_{1 i}+\left(1-\delta_{0 i}-\delta_{1 i}\right)\left[1-\Phi\left(\frac{1-\mu_{i}}{\sigma_{i}}\right)^{\alpha}\right]\right\}^{I_{1 i}} \\
& \times\left\{\left(1-\delta_{0 i}-\delta_{1 i}\right)\left[\frac{\alpha}{\sigma} \phi\left(\frac{y_{i}-\mu_{i}}{\sigma_{i}}\right) \Phi\left(\frac{y_{i}-\mu_{i}}{\sigma_{i}}\right)^{\alpha-1}\right]\right\}^{1-I_{0 i}-I_{1 i}} .
\end{aligned}
$$

Assim como no modelo tobit normal, os modelos mais simples podem ser obtidos de forma direta. Para o Tobit normal-potência duplamente censurado e inflacionado de zero, basta tomar $\delta_{1 i}=0$, para $i=1, \ldots, n$ e utilizar $h_{0}=h$ do tipo $h:(0,1) \rightarrow \mathbb{R}$. Para o Tobit normal-potência duplamente censurado e inflacionado de um, tome $\delta_{0 i}=0$, para $i=1, \ldots, n$ e $h_{1}=h$ do tipo $h:(0,1) \rightarrow \mathbb{R}$. Podemos ainda utilizar o modelo Tobit normal-potência duplamente censurado sem inflação, tomando $\delta_{0 i}=\delta_{1 i}=0$, para $i=1, \ldots, n$. É possível ainda utilizar a adaptação ao modelo de Cragg (1971), da mesma forma apresentada para o modelo tobit normal duplamente censurado e inflacionado.

\subsection{Inferência Bayesiana}

$\mathrm{Na}$ abordagem Bayesiana de modelos de regressão, estamos interessados em obter as distribuições marginais a posteriori dos parâmetros do modelo e uma forma eficiente de amostrar dados destas distribuições. Com isso, evitamos o uso de aproximações assintóticas e conseguimos obter estimações práticas de funções dos parâmetros por meio de técnicas de MCMC.

\subsubsection{Prioris para os parâmetros}

Para os modelos apresentados temos os vetores de parâmetros associados as covariáveis dos modelos tais que $\boldsymbol{\beta} \in \mathbb{R}^{p}, \boldsymbol{\rho} \in \mathbb{R}^{m_{0}}$ e $\boldsymbol{\gamma} \in \mathbb{R}^{m_{1}}$. Desta forma, é razoável atribuir para os vetores $\boldsymbol{\beta}, \boldsymbol{\rho}$ e $\gamma$ prioris contínuas e que estejam definidas em $\mathbb{R}^{p}, \mathbb{R}^{m_{0}}$ e $\mathbb{R}^{m_{1}}$ respectivamente, de modo que todo o espaço paramétrico seja pela priori atribuída ao parâmetro. Distribuições Normais ou t-Student 
multivariadas são algumas opções que satisfazem estas condições. A escolha da distribuição normal como priori é bastante comum na literatura devido a sua tratabilidade algébrica e por apresentar alguns resultados para distribuições condicionais já conhecidos. Desta forma, podemos optar por exemplo pelas prioris

$$
\begin{aligned}
& \boldsymbol{\beta} \sim N_{p}\left(0, \boldsymbol{\Sigma}_{\boldsymbol{p}}\right), \\
& \boldsymbol{\rho} \sim N_{m_{0}}\left(0, \boldsymbol{\Sigma}_{\boldsymbol{m}_{\mathbf{0}}}\right), \\
& \boldsymbol{\gamma} \sim N_{m_{1}}\left(0, \boldsymbol{\Sigma}_{\boldsymbol{m}_{\mathbf{1}}}\right),
\end{aligned}
$$

onde $\boldsymbol{\Sigma}_{\boldsymbol{p}}, \boldsymbol{\Sigma}_{\boldsymbol{m}_{\mathbf{0}}}$ e $\boldsymbol{\Sigma}_{\boldsymbol{m}_{\mathbf{1}}}$ são matrizes de dimensões $p, m_{0}$ e $m_{1}$, respectivamente, com uma estrutura de variância e covariância estabelecida. Quanto maiores os elementos da diagonal destas matrizes, menos informativas são as prioris e maior será o peso da verossimilhança da distribuição a posteriori dos parâmetros. Se estabelecemos os elementos fora das diagonais como iguais a zero, temos prioris independentes, o que seria equivalente a

$$
\begin{aligned}
\beta_{j} \sim N\left(0, \sigma_{\beta}^{2}\right), & \text { para } j=1, \ldots, p, \\
\rho_{j} \sim N\left(0, \sigma_{\rho}^{2}\right), & \text { para } j=1, \ldots, m_{0}, \\
\gamma_{j} \sim N\left(0, \sigma_{\gamma}^{2}\right), & \text { para } j=1, \ldots, m_{1} .
\end{aligned}
$$

É possível ainda estabelecer uma hierarquia onde $\boldsymbol{\Sigma}_{\boldsymbol{p}}$ depende do parâmetro de precisão da componente contínua do modelo ( $\phi$ para o modelo beta inflacionado e $\sigma$ para o modelo Tobit). Os modelos apresentaram comportamento bem robusto a escolha das prioris para os parâmetros ligados as covariáveis do modelo, de modo que os resultados pouco se alteraram para mudanças nas variâncias das distribuições a priori e assim como utilizando ou não dependência do parâmetro de dispersão. Optamos por uma escolha mais parcimoniosa utilizando variâncias grandes e independentes entre os parâmetros.

Para os parâmetros de precisão, temos $\sigma, \phi>0$ e portanto é necessário atribuir prioris definidas em $\mathbb{R}^{+}$. Nesta condição temos algumas opções como distribuições log-normal ou gama. Em modelos de regressão usuais, é comum o uso de priori $\operatorname{Gama}\left(v_{1}, v_{2}\right)$ para a precisão $1 / \sigma$ devido a conjugação com a distribuição normal. No contexto de modelos beta, da-Silva et al. (2011) também sugerem o uso de priori $\operatorname{Gama}\left(v_{1}, v_{2}\right)$ para o parâmetro de precisão $\phi$ e discutem a escolha dos hyperparâmetros $v_{1}$ e $v_{2}$ afim de obter uma priori vaga e ao mesmo tempo tratável computacionalmente, chegando aos valores $v_{1}=1$ e $v_{2}=0,033$ obtendo uma distribuição com a moda em zero e variância igual a 918, 27. Desta forma, podemos utilizar

$$
\begin{aligned}
& \frac{1}{\sigma} \sim \operatorname{gama}\left(v_{1}, v_{2}\right), \quad \text { ou } \\
& \phi \sim \operatorname{gama}\left(v_{1}, v_{2}\right) .
\end{aligned}
$$

Quando se tem uma noção intervalar do valor verdadeiro do parâmetro de precisão, é possível utilizar uma priori restrita a este intervalo, como $U(a, b)$. Esta opção pode ser interessante para reduzir o custo computacional, no entanto esta opção, além de contemplar todo o espaço paramétrico, não funcionou em alguns modelos devido a problemas de identificabilidade na função de verossimilhança.

Para o parâmetro de assimetria $\alpha>0$ do modelo Tobit normal-potência duplamente censurado, podemos seguir a mesma lógica aplicada aos parâmetros de precisão $\sigma$ e $\phi$. Para os modelos em que o parâmetro de precisão é também associado a um conjunto de covariáveis, como nas equações $(3.4),(3.7)$ e (3.9), podemos utilizar para o vetor $\boldsymbol{\omega}$ a mesma linha de prioris atribuídas para os vetores $\boldsymbol{\beta}, \boldsymbol{\rho}$ e $\boldsymbol{\gamma}$. 


\subsubsection{Obtenção das distribuições a posteriori para os parâmetros}

Seja $\pi(\boldsymbol{\theta})$ a distribuição a priori conjunta dos parâmetros $\boldsymbol{\theta}$, ou seja para o modelo de regressão $B I Z U$ temos

$$
\pi(\boldsymbol{\theta})=\pi(\boldsymbol{\beta}) \pi(\boldsymbol{\rho}) \pi(\boldsymbol{\gamma}) \pi(\phi),
$$

para o modelo tobit normal duplamente censurado e inflacionado temos

$$
\pi(\boldsymbol{\theta})=\pi(\boldsymbol{\beta}) \pi(\boldsymbol{\rho}) \pi(\boldsymbol{\gamma}) \pi(\sigma),
$$

e para o modelo tobit normal-potência duplamente censurado e inflacionado temos

$$
\pi(\boldsymbol{\theta})=\pi(\boldsymbol{\beta}) \pi(\boldsymbol{\rho}) \pi(\boldsymbol{\gamma}) \pi(\sigma) \pi(\alpha) .
$$

Para a análise inferencial do modelo, estamos interessado em obter as distribuição a posteriori dos parâmetros. A distribuição a posteriori conjunta, $\pi(\boldsymbol{\theta} \mid \boldsymbol{y})$, é obtida através da regra de Bayes e pode ser expressada da forma

$$
\pi(\boldsymbol{\theta} \mid \boldsymbol{y}) \propto L(\boldsymbol{\theta} ; \boldsymbol{y}) \times \pi(\boldsymbol{\theta}) .
$$

As distribuições a posteriori condicionais completas no modelo $B I Z U$ dadas por

$$
\begin{aligned}
& \pi(\boldsymbol{\beta} \mid \boldsymbol{\rho}, \boldsymbol{\gamma}, \phi, \boldsymbol{y}) \propto L_{1}(\boldsymbol{\beta}, \phi) \times \pi(\boldsymbol{\beta}) \\
& \pi(\phi \mid \boldsymbol{\beta}, \boldsymbol{\rho}, \boldsymbol{\gamma}, \boldsymbol{y}) \propto L_{1}(\boldsymbol{\beta}, \phi) \times \pi(\phi) \\
& \pi(\boldsymbol{\rho} \mid \boldsymbol{\beta}, \boldsymbol{\gamma}, \phi, \boldsymbol{y}) \propto L_{2}(\boldsymbol{\rho}, \boldsymbol{\gamma}) \times \pi(\boldsymbol{\rho}) \\
& \pi(\boldsymbol{\gamma} \mid \boldsymbol{\beta}, \boldsymbol{\rho}, \phi, \boldsymbol{y}) \propto L_{2}(\boldsymbol{\rho}, \boldsymbol{\gamma}) \times \pi(\boldsymbol{\gamma})
\end{aligned}
$$

Para o modelo Tobit normal duplamente censurado, temos forma semelhante para as condicionais completas, como por exemplo

$$
\pi(\sigma \mid \boldsymbol{\beta}, \boldsymbol{\rho}, \boldsymbol{\gamma}, \boldsymbol{y}) \propto L(\boldsymbol{\beta}, \boldsymbol{\rho}, \boldsymbol{\gamma}, \sigma) \times \pi(\sigma) .
$$

E para o modelo Tobit normal-potência duplamente censurado, temos forma semelhante com o acréscimo da distribuição

$$
\pi(\alpha \mid \boldsymbol{\beta}, \boldsymbol{\rho}, \boldsymbol{\gamma}, \sigma, \boldsymbol{y}) \propto L(\boldsymbol{\beta}, \boldsymbol{\rho}, \boldsymbol{\gamma}, \sigma, \alpha) \times \pi(\alpha) .
$$

Nota-se que nos modelos $\pi(\boldsymbol{\theta} \mid \boldsymbol{y})$ e as distribuições condicionais completas não podem ser expresso de forma fechada devido a presença de componentes discreto e contínuo. Uma alternativa é o uso de amostradores via metodologia MCMC.

O algoritmo de Metropolis-Hastings (Metropolis et al., 1953; Hastings, 1970) traz uma eficiente metologia de amostragem. Considere $\theta_{k}$ como um elemento do conjunto de parâmetros $\boldsymbol{\theta}$. Para obter uma amostra de tamanho $M$ da distribuição a posteriori $\pi\left(\theta_{k} \mid \boldsymbol{y}\right)$, no passo $1<j<M$, amostra-se $\theta_{k}^{*}$ da densidade de transição proposta $q_{k}\left(\theta_{k}^{(j)}, \theta_{k}^{*}\right)$ e o valor é aceito alocando $\theta_{k}^{(j+1)}=\theta_{k}^{*}$ com probabilidade

$$
\min \left\{1, \frac{\pi_{k}\left(\theta_{k}^{*}\right) / q_{k}\left(\theta_{k}^{(j)}, \theta_{k}^{*}\right)}{\pi_{k}\left(\theta_{k}^{(j)}\right) / q_{k}\left(\theta_{k}^{*}, \theta_{k}^{(j)}\right)}\right\},
$$

onde $\pi_{k}\left(\theta_{k}\right)=\pi\left(\theta_{k} \mid \boldsymbol{\theta}_{(-k)}, \boldsymbol{y}\right)$ corresponde a densidade condicional completa de $\theta_{k}$.

A dificuldade encontra-se em obter uma distribuição geradora de candidatos $q(.,$.$) eficiente.$ Uma alternativa é utilizar um passeio aleatório. Se $\theta_{k} \in \mathbb{R}^{q}$, podemos utilizar

$$
q_{k}\left(\theta_{k}^{(j)}, \theta_{k}^{*}\right)=N_{q}\left(\theta_{k}^{(j)}, v_{\theta_{k}}\right)
$$

no caso $\theta_{k}>0$, podemos optar em amostrar $\log \left(\theta_{k}\right)$. A variância $v_{\theta_{k}}$ é determinada de modo a que se obtenha uma taxa de aceitação desejada. Uma opção é utilizar $v_{\theta_{k}}=c \hat{\boldsymbol{I}}^{-1}\left(\theta_{k}^{(j)}\right)$, onde $\hat{\boldsymbol{I}}^{-1}\left(\theta_{k}^{(j)}\right)$ é 
a inversa da informação de Fisher observada em $\theta_{k}^{(j)}$ e $c$ é uma constante de calibração. O software OpenBugs também possui um algoritmo eficiente de $\mathrm{M}-\mathrm{H}$, onde podemos entrar diretamente com a função de verossimilhança e atribuir:

$$
\text { dummy }[i] \sim \log l i k(\log \operatorname{Like}[i]),
$$

onde $\log L i k e[i]$ recebe o logaritmo da função de verossimilhança.

As duas formas de construir o algoritmo apresentaram resultados de estimação semelhantes. No entanto o procedimento via OpenBugs reduziu o custo computacional pela metade e possui uma implementação computacional mais simples.

\subsection{Medidas de diagnóstico}

Esta parte da análise consiste em verificar possíveis afastamento das suposições assumidas na modelagem dos dados. É importante detectar a presença de valores extremos e verificar seu impacto nas conclusões inferenciais do modelo. Análises gráficas contribuem para avaliar a estabilidade do modelo e ajudam a mensurar a distância entre os valores reais e os valores do modelo ajustado.

Quando se tem mais de um modelo candidato para descrever o comportamento do conjunto de dados, é importante construir uma metodologia de escolha do modelo mais adequado ao conjunto de dados. Sendo assim, a análise de diagnóstico tem um papel fundamental para auxiliar o pesquisador na definição de um modelo que descreva corretamente o fenômeno representado pelo conjunto de dados.

\subsubsection{Critérios de escolha do modelo}

Para critério de escolha do modelo mais adequado ao conjunto de dados, vamos utilizar como base a medida Deviance, dada por

$$
D(\boldsymbol{\theta})=-2 \sum_{i=1}^{n} \log \left[f\left(y_{i} \mid \boldsymbol{\theta}\right)\right],
$$

que pode ser interpretada como a log-verossimilhança observada a posteriori. Considere também, $\boldsymbol{\theta}^{(j)}$ como o j-ésimo elemento de amostra da distribuição a posteriori de $(\boldsymbol{\theta} \mid \boldsymbol{y}), j \in\{1, \ldots, M\}$, e $\bar{D}=$ $\sum_{j=1}^{M} D\left(\boldsymbol{\theta}^{(j)}\right) / M$. Com base nessas medidas temos o critério $D I C$ proposto por Spiegelhalter et al. (2002). Este critério pode ser estimado via Monte Carlo utilizando a amostra da distribuição a posteriori do conjunto de parâmetros, $\pi(\boldsymbol{\theta} \mid \boldsymbol{y})$, da forma

$$
\widehat{D I C}=\bar{D}+\hat{\rho_{D}}
$$

onde $\rho_{D}$ corresponde ao número efetivo de parâmetros,

$$
\rho_{D}=E_{\boldsymbol{\theta} \mid \boldsymbol{y}}\{D(\boldsymbol{\theta})\}-D\left(E_{\boldsymbol{\theta} \mid \boldsymbol{y}}\{\boldsymbol{\theta}\}\right),
$$

e sua estimativa também pode ser obtida via Monte Carlo. Os critérios seguintes são adaptações do critério de informação de Akaike $(A I C)$ e do critério de informação Bayesiano de Schwarz $(B I C)$ para o contexto de inferência Bayesiana. Os critérios são chamados de $E A I C$ e $E B I C$, respectivamente e são obtidos da forma

$$
\widehat{E A I C}=\bar{D}+2 \vartheta, \quad \text { e } \widehat{E B I C}=\bar{D}+\vartheta \log (n)
$$

onde $\vartheta$ corresponde ao número de parâmetros do modelo. Pelos três critérios, o melhor ajuste é considerado pela menor estatística. Observe que a diferença entre estas três medidas está na penalização pela quantidade de parâmetros do modelo. Enquanto o DIC penaliza a Deviance pelo número efetivo de parâmetros do modelo, $\rho_{D}$, a medida $E A I C$ penaliza pela quantidade fixa de 
parâmetros no modelo e a medida $E B I C$ faz esta penalização levando em conta o tamanho da amostra.

Outro critério é considerado com base no $C P O_{i}=p\left(y_{i} \mid \boldsymbol{y}_{(-i)}\right)$ (conditional predictive ordinate), expresso da forma

$$
C P O_{i}=\int_{\boldsymbol{\theta} \in \Theta} f\left(y_{i} \mid \boldsymbol{\theta}\right) \pi\left(\boldsymbol{\theta} \mid \boldsymbol{y}_{(-i)}\right) d \boldsymbol{\theta}=\left\{\int_{\boldsymbol{\theta} \in \Theta} \frac{\pi(\boldsymbol{\theta} \mid \boldsymbol{y})}{f\left(y_{i} \mid \boldsymbol{\theta}\right)} d \boldsymbol{\theta}\right\}
$$

onde $\boldsymbol{y}_{(-i)}$ corresponde ao conjunto de dados sem a $i$-ésima observação para $i=1, \ldots, n$. O $C P O_{i}$ pode ser interpretado como a altura da densidade marginal de $y_{i}$ e pode ser estimado via Monte Carlo utilizando uma única amostra MCMC obtida da posteriori $\pi(\boldsymbol{\theta} \mid \boldsymbol{y})$ (vide, Dey et al., 1997).

$$
\widehat{C P O}_{i}=\left\{\frac{1}{M} \sum_{j=1}^{M} \frac{1}{f\left(y_{i} \mid \theta^{(j)}\right)}\right\}^{-1},
$$

e com ele é calculada a medida a log-pseudo verossimilhança marginal, $L M P L=\sum_{i=1}^{n}=\log \left(\widehat{C P O}_{i}\right)$, em que o melhor ajuste é considerado pela maior estatística.

\subsubsection{Resíduos}

Uma análise de resíduos podem ajudar a verificar a adequabilidade do modelo e inclusive a robustez dos resultados inferenciais. Análises gráficas dos resíduos pelas observações ou pelos valores preditos, principalmente em suas versões padronizadas nos permitem verificar a estabilidade do modelo e nos trazem certa sensibilidade para detectar valores atípicos no conjunto de dados.

A análise de resíduos pode ser feita com base nos resíduos Bayesianos padronizados sugeridos por Paolino et al. (2003), que correspondem às observações subtraídas de seu valor esperado a posteriori e dividida pelo seu desvio padrão da sua distribuição a posteriori.

$$
r_{i}=\frac{y_{i}-E\left(y_{i} \mid \boldsymbol{y}\right)}{\sqrt{\operatorname{Var}\left(y_{i} \mid \boldsymbol{y}\right)}}, \quad i=1 \ldots, n
$$

onde,

$$
\begin{aligned}
E\left(y_{i} \mid \boldsymbol{y}\right) & =\int E\left(y_{i} \mid \boldsymbol{\theta}\right) \pi(\boldsymbol{\theta} \mid \boldsymbol{y}) d \boldsymbol{\theta} \\
E\left(y_{i}^{2} \mid \boldsymbol{y}\right) & =\int\left\{\operatorname{Var}\left(y_{i} \mid \boldsymbol{\theta}\right)+E\left(y_{i} \mid \boldsymbol{\theta}\right)^{2}\right\} \pi(\boldsymbol{\theta} \mid \boldsymbol{y}) d \boldsymbol{\theta} \\
\operatorname{Var}\left(y_{i} \mid \boldsymbol{y}\right) & =E\left(y_{i}^{2} \mid \boldsymbol{y}\right)-\left[E\left(y_{i} \mid \boldsymbol{y}\right)\right]^{2} .
\end{aligned}
$$

Uma vez que é obtido um amostrador da distribuição a posteriori dos parâmetros, $\pi(\boldsymbol{\theta} \mid \boldsymbol{y})$, as integrais acima podem ser facilmente obtidas via integração Monte-Carlo, da forma:

$$
\int f(\boldsymbol{\theta}) d \boldsymbol{\theta}=\sum_{j=1}^{M} \frac{f\left(\boldsymbol{\theta}_{j}\right)}{M},
$$

onde $\boldsymbol{\theta}_{j}$ corresponde ao $j$-ésimo elemento da amostra obtida de $\pi(\boldsymbol{\theta} \mid \boldsymbol{y})$.

Os gráficos de $r_{i}$ pelas observações ou pela preditiva $E\left(y_{i} \mid \boldsymbol{y}\right)$ podem ajudar a detectar a presença de alguma tendência não especificada no modelo (como variável omitida, evidência de nãolinearidade) e presença de heterocedasticidade. Os gráficos de $r_{i}$ pelas variáveis exógenas do modelo podem apontar alguma correlação entre erro e variável explicativa, indicando a necessidade do uso de variáveis instrumentais.

Devido a presença de componentes discreta e contínua no modelo, os resíduos padronizados nem sempre apresentaram um bom comportamento e por isso podemos, também, trabalhar com os 
resíduos Bayesianos padronizados condicionais, $r c_{i}^{(0)}, r c_{i}^{(1)}$ e $r c_{i}^{(c)}$, onde:

$$
\begin{aligned}
& r c_{i}^{(0)}=\frac{1-P\left(y_{i}=0 \mid y_{i} \in\{0,1\}, \boldsymbol{y}\right)}{\sqrt{\operatorname{Var}\left(y_{i} \mid y_{i} \in\{0,1\}, \boldsymbol{y}\right)}}, \\
& r c_{i}^{(1)}=\frac{1-P\left(y_{i}=1 \mid y_{i} \in\{0,1\}, \boldsymbol{y}\right)}{\sqrt{\operatorname{Var}\left(y_{i} \mid y_{i} \in\{0,1\}, \boldsymbol{y}\right)}}, \\
& r c_{i}^{(c)}=\frac{y_{i}-E\left(y_{i} \mid y_{i} \in(0,1), \boldsymbol{y}\right)}{\sqrt{\operatorname{Var}\left(y_{i} \mid y_{i} \in(0,1), \boldsymbol{y}\right)}},
\end{aligned}
$$

e obtemos

$$
r c_{i}= \begin{cases}r c_{i}^{(0)} & \text { se } y_{i}=0, \\ r c_{i}^{(1)} & \text { se } y_{i}=1, \\ r c_{i}^{(c)} & \text { se } y_{i} \in(0,1)\end{cases}
$$

Com os resíduos Bayesianos condicionais, estamos avaliado separadamente a estabilidade submodelo do componente discreto e do componente contínuo. Observe que $r c_{i}^{(0)}$ corresponde ao resíduo Bayesiano padronizado do sub-modelo do componente discreto que modela a ocorrência de zeros e $r c_{i}^{(1)}$ corresponde ao resíduo Bayesiano padronizado do sub-modelo do componente discreto que modela a ocorrência de uns. Desta forma, é razoável utilizar dois gráficos para a componente discreta do modelo, o primeiro com $r c_{i}^{(0)}$ contra $P\left(y_{i}=0 \mid \boldsymbol{y}\right)$ e o segundo de $r c_{i}^{(1)}$ contra $P\left(y_{i}=1 \mid \boldsymbol{y}\right)$, para $i=1, \ldots, n$. Para a componente contínua podemos obter o gráfico de $r c_{i}^{(c)}$ contra $E\left(y_{i} \mid y_{i} \in(0,1), \boldsymbol{y}\right)$. Estes gráficos ajudam a detectar pontos marginalmente aberrantes em cada sub-modelo. No caso dos modelos com inflação de um único valor $c(c=0$ ou $c=1)$, construímos os resíduos Bayesianos condicionais da forma

$$
\begin{aligned}
r c_{i}^{(d)} & =\frac{1-P\left(y_{i}=c \mid \boldsymbol{y}\right)}{\sqrt{P\left(y_{i}=c \mid \boldsymbol{y}\right)\left[1-P\left(y_{i}=c \mid \boldsymbol{y}\right)\right]}}, \\
r c_{i}^{(c)} & =\frac{y_{i}-E\left(y_{i} \mid y_{i} \in(0,1), \boldsymbol{y}\right)}{\sqrt{\operatorname{Var}\left(y_{i} \mid y_{i} \in(0,1), \boldsymbol{y}\right)}}
\end{aligned}
$$

e obtemos

$$
r c_{i}= \begin{cases}r c_{i}^{(d)} & \text { se } y_{i}=c, \\ r c_{i}^{(c)} & \text { se } y_{i} \in(0,1) .\end{cases}
$$

Os gráficos dos resíduos Bayesianos condicionais em geral possuem distribuição empírica assimétrica dificultando o uso de medidas usuais de diagnóstico. Os gráficos de $r c_{i}$ contra $E\left(y_{i} \mid \boldsymbol{y}\right)$ podem ajudar a detectar pontos globalmente aberrantes ou influentes.

Ospina (2008) também sugere para a análise de resíduo dos modelos beta inflacionado o uso de resíduos ponderados, que pode ser facilmente adaptada ao contexto dos resíduos Bayesianos e utilizado nos demais modelos. Os resíduos Bayesianos ponderados podem ser expressos da forma

$$
r p_{i}=E\left[\delta_{0 i} \mid \boldsymbol{y}\right] r c_{i}^{(0)}+E\left[\delta_{1 i} \mid \boldsymbol{y}\right] r c_{i}^{(1)}+\left(1-E\left[\delta_{0 i} \mid \boldsymbol{y}\right]-E\left[\delta_{1 i} \mid \boldsymbol{y}\right]\right) r c_{i}^{(c)} .
$$

Os gráficos de $r p_{i}$ contra $E\left(y_{i} \mid \boldsymbol{y}\right)$ pode ajudar a detectar o posicionamento de pontos atípicos no conjunto de dados.

\subsubsection{Pontos influentes}

A detecção de pontos influentes é outro aspecto importante na análise de diagnóstico. Isto é, desejamos encontrar aqueles pontos que exercem um peso desproporcional nas estimativas dos parâmetros do modelo e então verificar o seu impacto nos resultados inferenciais.

Utilizaremos algumas medidas de influência para modelos de regressão Bayesianos (ver Weiss e Cook, 1992 e Peng e Dey, 1995). 
Para a análise de diagnóstico considerando deleção de casos, consideremos a função de perturbação introduzida por Weiss (1996). Seja então $\pi\left[\boldsymbol{\theta} \mid \boldsymbol{y}_{(-i)}\right]$ e $\pi[\boldsymbol{\theta} \mid \boldsymbol{y}]$ as distribuições a posteriori de $\boldsymbol{\theta}$ sem a $i$-ésima observação e com todos os dados, respectivamente, então a função de perturbação é definida por

$$
m_{i}(\boldsymbol{\theta})=\frac{\pi\left[\boldsymbol{\theta} \mid \boldsymbol{y}_{(-i)}\right]}{\pi[\boldsymbol{\theta} \mid \boldsymbol{y}]}
$$

Além disso, consideraremos a medida de divergência-g entre duas densidades $\pi_{1}$ e $\pi_{2}$ avaliada em $\boldsymbol{\theta}$ sugerida por Csiszár (1967), que é dada por

$$
d_{g}\left(\pi_{1}, \pi_{2}\right)=E_{\boldsymbol{\theta} \mid \boldsymbol{y}}\left\{g\left(\frac{\pi_{1}(\boldsymbol{\theta})}{\pi_{2}(\boldsymbol{\theta})}\right)\right\}
$$

onde que $g($.$) é uma função convexa tal que g(1)=0$. É possível obter algumas medidas de divergências específicas, considerando particulares funções $g($.$) .$

- Divergência de Kullback-Leibler $\left(K\left(\pi_{1}, \pi_{2}\right)\right)$.

Esta medida é obtida quando $g\left(\pi_{1}, \pi_{2}\right)=-\log \left(\frac{\pi_{1}}{\pi_{2}}\right)$. Para o caso de interesse de deleção de casos, com a função de perturbação definida em (3.10), devemos considerar $\pi_{1}=\pi\left[\boldsymbol{\theta} \mid \boldsymbol{y}_{(-i)}\right] \mathrm{e}$ $\pi_{2}=\pi[\boldsymbol{\theta} \mid \boldsymbol{y}]$.

Desta forma, temos a medida de influência

$$
K\left(\pi\left[\boldsymbol{\theta} \mid \boldsymbol{y}_{(-i)}\right], \pi[\boldsymbol{\theta} \mid \boldsymbol{y}]\right)=E_{\boldsymbol{\theta} \mid \boldsymbol{y}}\left\{-\log \left(\frac{\pi\left[\boldsymbol{\theta} \mid \boldsymbol{y}_{(-i)}\right]}{\pi[\boldsymbol{\theta} \mid \boldsymbol{y}]}\right)\right\}
$$

- Distância-J $\left(J\left(\pi_{1}, \pi_{2}\right)\right)$.

Esta medida é obtida quando

$$
g\left(\pi_{1}, \pi_{2}\right)=\left(\frac{\pi_{1}}{\pi_{2}}-1\right) \times \log \left(\frac{\pi_{1}}{\pi_{2}}\right)
$$

e pode ser vista como uma versão simétrica da divergência de Kullback-Leibler.

Assim, temos

$$
J\left(\pi\left[\boldsymbol{\theta} \mid \boldsymbol{y}_{(-i)}\right], \pi[\boldsymbol{\theta} \mid \boldsymbol{y}]\right)=E_{\boldsymbol{\theta} \mid \boldsymbol{y}}\left\{\left(\frac{\pi\left[\boldsymbol{\theta} \mid \boldsymbol{y}_{(-i)}\right]}{\pi[\boldsymbol{\theta} \mid \boldsymbol{y}]}-1\right) \times \log \left(\frac{\pi\left[\boldsymbol{\theta} \mid \boldsymbol{y}_{(-i)}\right]}{\pi[\boldsymbol{\theta} \mid \boldsymbol{y}]}\right)\right\}
$$

- Distância-L $\left(L\left(\pi_{1}, \pi_{2}\right)\right)$

Esta medida é obtida quando

$$
g\left(\pi_{1}, \pi_{2}\right)=\left|\frac{\pi_{1}}{\pi_{2}}-1\right|
$$

Assim, temos

$$
L\left(\pi\left[\boldsymbol{\theta} \mid \boldsymbol{y}_{(-i)}\right], \pi[\boldsymbol{\theta} \mid \boldsymbol{y}]\right)=E_{\boldsymbol{\theta} \mid \boldsymbol{y}}\left\{\left|\frac{\pi\left[\boldsymbol{\theta} \mid \boldsymbol{y}_{(-i)}\right]}{\pi[\boldsymbol{\theta} \mid \boldsymbol{y}]}-1\right|\right\}
$$


- Distância- $\chi^{2}\left(\chi^{2}\left(\pi_{1}, \pi_{2}\right)\right)$.

Esta medida é obtida quando

$$
g\left(\pi_{1}, \pi_{2}\right)=\left(\frac{\pi_{1}}{\pi_{2}}-1\right)^{2}
$$

Assim, temos

$$
\chi^{2}\left(\pi\left[\boldsymbol{\theta} \mid \boldsymbol{y}_{(-i)}\right], \pi[\boldsymbol{\theta} \mid \boldsymbol{y}]\right)=E_{\boldsymbol{\theta} \mid \boldsymbol{y}}\left\{\left(\frac{\pi\left[\boldsymbol{\theta} \mid \boldsymbol{y}_{(-i)}\right]}{\pi[\boldsymbol{\theta} \mid \boldsymbol{y}]}-1\right)^{2}\right\} .
$$

Garay et al. (2012) discute a escolha de um ponto de corte para essas medidas baseado na proposta apresentada por Peng e Dey (1995). O ponto de corte é função de uma probabilidade $p$ que cresce na medida em que $p$ se afasta de $1 / 2$. Esta função é dada por

$$
d_{g}(p)=\frac{g(2 p)+g(2(1-p))}{2} .
$$

Para $p=0,8$, por exemplo, um ponto é considerado influente pela divergência de KullbackLeibler quando $d_{K-L} \geq 0,22$.

\subsection{Estudo de simulação}

Com o objetivo de avaliar a robustez entre diferentes percentuais de inflação do conjunto de dados, assim como a competitividade entre os modelos beta inflacionado e Tobit duplamente censurado e inflacionado foram feitos alguns estudos comparativos entre os dois modelos. O primeiro estudo consiste em avaliar o desempenho dos dois modelos quando temos um conjunto de dados gerado pela distribuição $B I Z U$ e o segundo estudo consiste em avaliar o desempenho dos modelos quando os dados seguem uma distribuição normal censurada em $[0,1]$ e inflacionada de zeros e uns (Tobit normal duplamente censurado e inflacionado).

\subsubsection{Estudo 1}

Nesta etapa foram gerados dados BIZU com percentual de inflação aproximadamente de $10 \%$, $25 \%$ e $50 \%$, divididas entre zeros e uns. Foi feito um estudo semelhante ao apresentado em Ospina (2008), onde foram gerada uma amostra de tamanho $n=500$ de $y_{i} \sim B I Z U\left(\delta_{0 i}, \delta_{1 i}, \mu_{i}, \phi\right)$, para $i=1, \ldots, n$. Foram consideras as funções de ligação

$$
\begin{aligned}
\log \left(\frac{\mu_{i}}{1-\mu_{i}}\right) & =\beta_{0}+\beta_{1} x_{i} \\
\log \left(\frac{\delta_{0 i}}{1-\delta_{0 i}-\delta_{1 i}}\right) & =\rho_{0}+\rho_{1} v_{i} \\
\log \left(\frac{\delta_{1 i}}{1-\delta_{0 i}-\delta_{1 i}}\right) & =\gamma_{0}+\gamma_{1} z_{i},
\end{aligned}
$$

onde $x_{i}, v_{i}$ e $z_{i}$ são realizações independentes de uma $U(0,1)$, e os valores verdadeiros dos parâmetros são:

- com inflação de $10 \%$ utilizamos $\rho_{0}=-2,5, \rho_{1}=-0,5, \gamma_{0}=-2,5, \gamma_{1}=-0,7, \beta_{0}=-1,5$, $\beta_{1}=1,5$ e $\phi=50$. A proporção de zeros é cerca de $6,6 \%$ a proporção de uns é cerca de $4,2 \%$.

- com inflação de $25 \%$ utilizamos $\rho_{0}=-2, \rho_{1}=0,1, \gamma_{0}=-2, \gamma_{1}=0,2, \beta_{0}=-1,5, \beta_{1}=1,5$ e $\phi=50$. A proporção de zeros é cerca de $10,8 \%$ a proporção de uns é cerca de $11 \%$. 
- com inflação de $50 \%$ utilizamos $\rho_{0}=-1, \rho_{1}=0,5, \gamma_{0}=-1, \gamma_{1}=0,7, \beta_{0}=-1,5, \beta_{1}=1,5$ e $\phi=50$. A proporção de zeros foi cerca de $23,2 \%$ a proporção de uns foi cerca de $24 \%$.

No procedimento de estimação dos modelos via MCMC foi considerado um burn-in de tamanho 10.000 e em seguida gerada uma amostra de tamanho 50.000 com saltos de tamanho 20. Foi também verificada a convergência da cadeia e baixo índice de autocorrelação. O procedimento foi realizado via OpenBugs.

Foram atribuídas prioris normal com média zero e variância $10^{3}$ para os parâmetros dos regressores e para os parâmetros de precisão utilizamos:

$$
\begin{aligned}
& \sigma \sim U(0,50), \quad \text { (para o modelo Tobit normal inflacionado) } \\
& \phi \sim U(0,100), \quad \text { (para o modelo BIZU) }
\end{aligned}
$$

Tabela 3.1: Comparação entre os modelos via DIC, $\widehat{E A I C}$, $\widehat{E B I C}$ e LMPL.

\begin{tabular}{l|l|cccc}
\hline Inflação & Modelo & DIC & $\widehat{E A I C}$ & $\widehat{E B I C}$ & LMPL \\
\hline $10 \%$ & BIZU & -799.67 & -792.69 & -763.19 & 399.76 \\
& Tobit inf. & -784.82 & -777.83 & -748.33 & 392.30 \\
\hline $25 \%$ & BIZU & -341.41 & -334.48 & -304.98 & 170.79 \\
& Tobit inf. & -327.43 & -320.48 & -290.98 & 163.79 \\
\hline $50 \%$ & BIZU & 454.66 & 461.66 & 491.16 & -227.40 \\
& Tobit inf. & 459.26 & 466.20 & 495.70 & -229.75 \\
\hline
\end{tabular}


Nota-se o modelo BIZU apresentou melhor desempenho pelos critérios DIC, $\widehat{E A I C}, \widehat{E B I C}$ e LMPL.

Para os dados com $10 \%$ de inflação foram obtidas as seguintes estimativas para os parâmetros:

Tabela 3.2: Estatísticas das distribuições marginais a posteriori dos parâmetros para o modelo BIZU.

\begin{tabular}{c|c|c|c|c}
\hline Parâmetros & Média & D.P. & Q 2,5\% & Q 97, 5\% \\
\hline$\beta_{0}$ & -1.522 & 0.030 & -1.582 & -1.462 \\
$\beta_{1}$ & 1.545 & 0.050 & 1.446 & 1.645 \\
$\rho_{0}$ & -1.876 & 0.311 & -2.504 & -1.288 \\
$\rho_{1}$ & -1.782 & 0.688 & -3.160 & -0.459 \\
$\gamma_{0}$ & -2.267 & 0.379 & -3.047 & -1.558 \\
$\gamma_{1}$ & -1.973 & 0.857 & -3.696 & -0.347 \\
$\phi$ & 52.76 & 3.508 & 46.13 & 59.84 \\
\hline
\end{tabular}

Tabela 3.3: Estatísticas das distribuições marginais a posteriori dos parâmetros para o modelo Tobit inflacionado.

\begin{tabular}{c|c|c|c|c}
\hline Parâmetros & Média & D.P. & Q 2,5\% & Q 97,5\% \\
\hline$\beta_{0}$ & -1.533 & 0.033 & -1.601 & -1.468 \\
$\beta_{1}$ & 1.561 & 0.052 & 1.458 & 1.667 \\
$\rho_{0}$ & -1.878 & 0.312 & -2.511 & -1.289 \\
$\rho_{1}$ & -1.788 & 0.685 & -3.170 & -0.473 \\
$\gamma_{0}$ & -2.281 & 0.389 & -3.104 & -1.567 \\
$\gamma_{1}$ & -1.940 & 0.875 & 3.667 & -0.177 \\
$\sigma$ & 0.063 & 0.002 & 0.059 & 0.067 \\
\hline
\end{tabular}

Nota-se que para ambos os modelos, todos os parâmetros estimados foram significativos e o intervalo de credibilidade de $95 \%$ contemplam os valores verdadeiros dos parâmetros. As estimativas ficaram bem similares entre os dois modelos, inclusive o desvio padrão a posteriori. As estimativas para os parâmetros correspondentes a componente contínua ficou bem próxima do valor verdadeiro e a estimativas correspondentes as componentes discretas ficaram um pouco afastadas do valor real e a estimativa de $\gamma_{1}$ não foi significativa no modelo Tobit, o que ocorre devido a baixa quantidade de zeros e uns no conjunto de dados.

Pelas Figuras 3.1 e 3.2 é possível notar a grande semelhança entre os resíduos dos dois modelos. A Figura 3.3 mostra que não foi detectado nenhum ponto influente nos modelos pela divergência de K-L. 

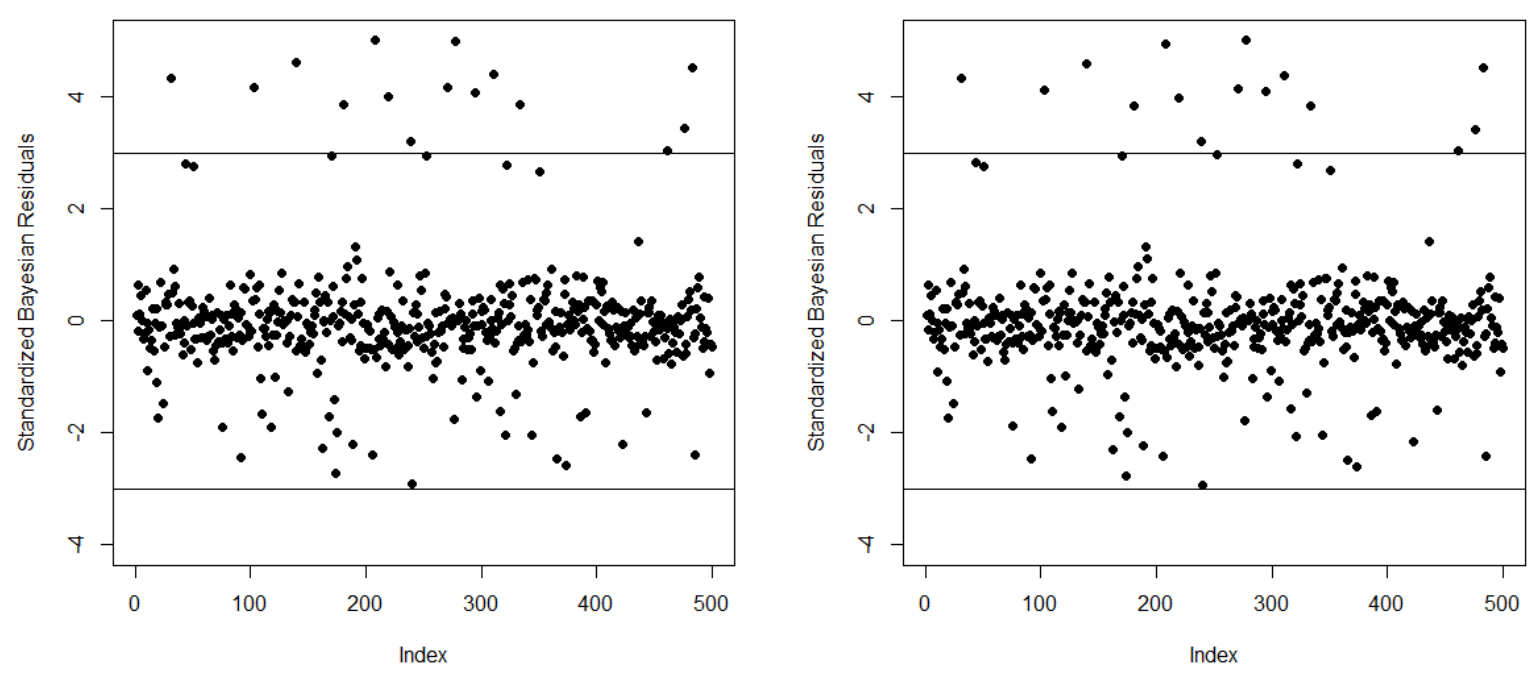

Figura 3.1: Gráfico dos resíduos Bayesianos padronizados pelas observações para os modelos BIZU (esquerda) e Tobit inflacionado (direita).
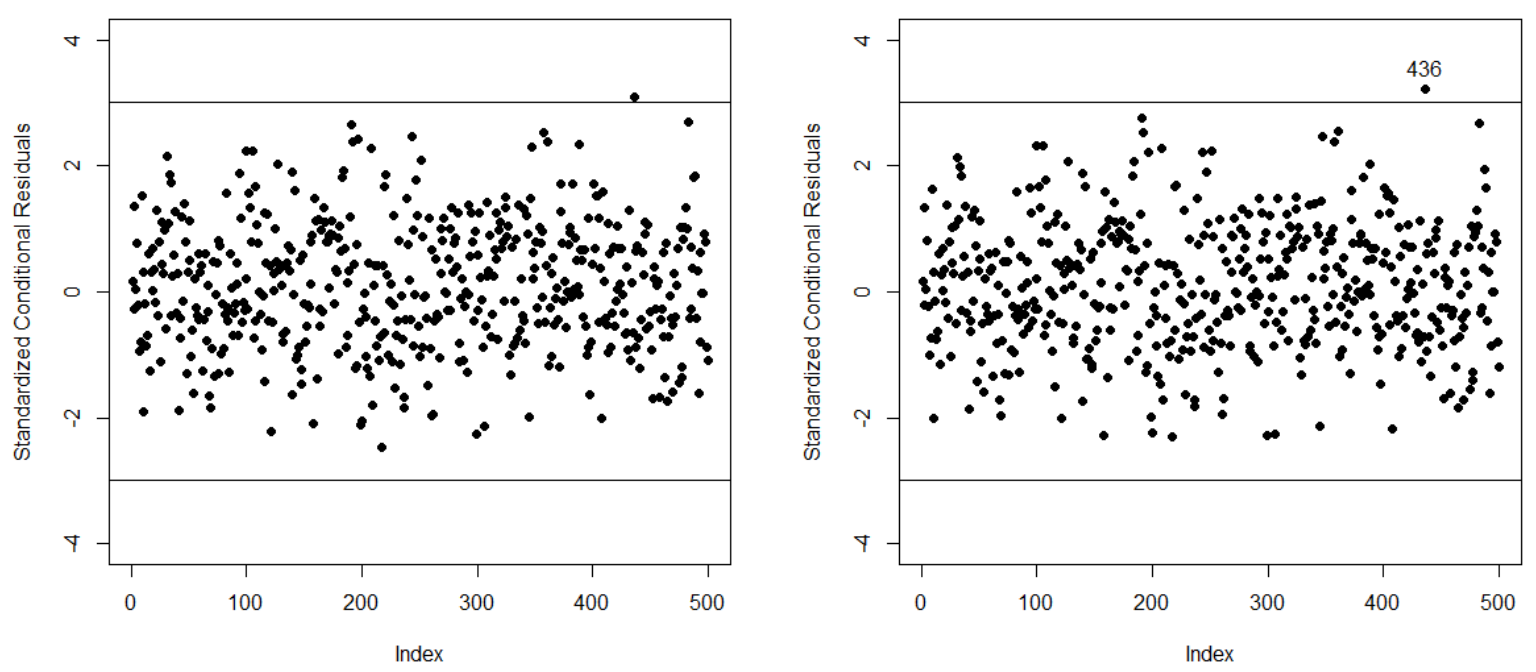

Figura 3.2: Gráfico dos resíduos Bayesianos condicionais padronizados pelas observações para os modelos BIZU (esquerda) e Tobit inflacionado (direita). 

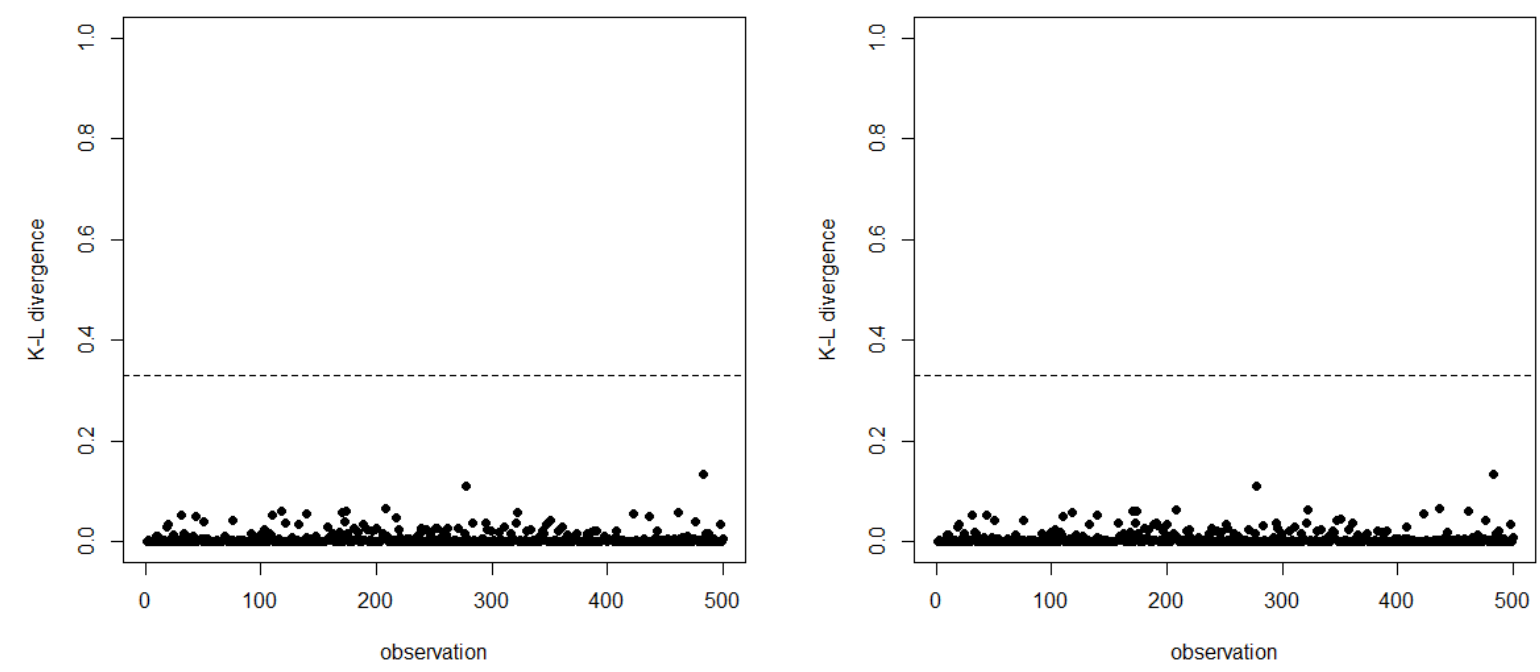

Figura 3.3: Gráfico da divergência de K-L para o modelo BIZU (esquerda) e Tobit inflacionado (direita). 


\subsubsection{Estudo 2}

Nesta etapa foram gerados dados Tobit-normal duplamente censurado com percentual de inflação de $10 \%, 25 \%$ e $50 \%$ e com censura em torno de $5 \%$ do conjunto de dados. Foi feito um estudo semelhante ao apresentado em Ospina (2008), onde foram gerada uma amostra de tamanho $n=500$ de $y_{i} \sim B I Z U\left(\delta_{0 i}, \delta_{1 i}, \mu_{i}, \phi\right)$, para $i=1, \ldots, n$. Foram consideras as funções de ligação

$$
\begin{aligned}
\log \left(\frac{\mu_{i}}{1-\mu_{i}}\right) & =\beta_{0}+\beta_{1} x_{i} \\
\log \left(\frac{\delta_{0 i}}{1-\delta_{0 i}-\delta_{1 i}}\right) & =\rho_{0}+\rho_{1} v_{i} \\
\log \left(\frac{\delta_{1 i}}{1-\delta_{0 i}-\delta_{1 i}}\right) & =\gamma_{0}+\gamma_{1} z_{i},
\end{aligned}
$$

onde $x_{i}, v_{i}$ e $z_{i}$ são realizações independentes de uma $U(0,1)$, e os valores verdadeiros dos parâmetros são:

- com inflação de $10 \%$ utilizamos $\rho_{0}=-2,5, \rho_{1}=-0,5, \gamma_{0}=-2,5, \gamma_{1}=-0,7, \beta_{0}=-1,5$, $\beta_{1}=1,5$ e $\sigma=0,2$. A proporção inflacionada de zeros é cerca de $6,8 \%$, a proporção de uns é cerca de $4,2 \%$ e a proporção de censura a esquerda foi de $5 \%$.

- com inflação de $25 \%$ utilizamos $\rho_{0}=-2, \rho_{1}=0,1, \gamma_{0}=-2, \gamma_{1}=0,2, \beta_{0}=-1,5, \beta_{1}=1,5$ e $\sigma=0,265$. A proporção inflacionada de zeros é cerca de $9 \%$, a proporção de uns é cerca de $12,4 \%$ e a proporção de censura foi de $8 \%$ a esquerda e $1 \%$ a direita.

- com inflação de $50 \%$ utilizamos $\rho_{0}=-1, \rho_{1}=0,5, \gamma_{0}=-1, \gamma_{1}=0,7, \beta_{0}=-1,5, \beta_{1}=1,5$ e $\sigma=0,316$. A proporção inflacionada de zeros foi cerca de $24,4 \%$ a proporção de uns foi cerca de $27,6 \%$ e a proporção de censura foi de $7,6 \%$ a esquerda e $0,6 \%$ a direita.

No procedimento de estimação dos modelos via MCMC foi considerado um burn-in de tamanho 10.000 e em seguida gerada uma amostra de tamanho 50.000 com saltos de tamanho 20. Foi também verificada a convergência da cadeia e baixo índice de autocorrelação. O procedimento foi realizado via OpenBugs.

Foram atribuídas prioris normal com média zero e variância $10^{3}$ para os parâmetros dos regressores e para os parâmetros de precisão utilizamos:

$$
\begin{gathered}
(1 / \sigma) \sim \operatorname{Gama}(1,0.1), \quad(\text { para o modelo tobit normal inflacionado }) \\
\phi \sim \operatorname{Gama}(1,0.1), \quad \text { (para o modelo BIZU) } .
\end{gathered}
$$


Tabela 3.4: Comparação entre os modelos via DIC, $\widehat{E A I C}, \widehat{E B I C}$ e LMPL.

\begin{tabular}{l|l|cccc}
\hline Inflação & Modelo & DIC & $\widehat{E A I C}$ & $\widehat{E B I C}$ & LMPL \\
\hline $10 \%$ & BIZU & 258.56 & 265.56 & 295.06 & -130.21 \\
& Tobit inf. & 215.47 & 229.13 & 258.63 & -112.06 \\
\hline $25 \%$ & BIZU & 685.82 & 692.77 & 722.27 & -343.50 \\
& Tobit inf. & 662.09 & 675.42 & 704.92 & -334.62 \\
\hline $50 \%$ & BIZU & 1035.39 & 1042.48 & 1071.98 & -518.09 \\
& Tobit inf. & 1024.19 & 1031.31 & 1060.81 & -512.49 \\
\hline
\end{tabular}

Nota-se o modelo tobit inflacionado apresentou melhor desempenho pelos critérios DIC, $\widehat{E A I C}$, $\widehat{E B I C}$ e LMPL. Para os dados com $10 \%$ de inflação foram obtidas as seguintes estimativas para os parâmetros:

Tabela 3.5: Estatísticas das distribuições marginais a posteriori dos parâmetros para o modelo BIZU.

\begin{tabular}{ccccc}
\hline Parâmetros & Média & D.P. & Q $2,5 \%$ & Q 97,5\% \\
\hline$\beta_{0}$ & -1.253 & 0.083 & -1.418 & -1.092 \\
$\beta_{1}$ & 1.214 & 0.135 & 0.950 & 1.48 \\
$\rho_{0}$ & -1.943 & 0.286 & -2.527 & -1.400 \\
$\rho_{1}$ & -0.067 & 0.491 & -1.027 & 0.903 \\
$\gamma_{0}$ & -2.680 & 0.425 & -3.557 & -1.889 \\
$\gamma_{1}$ & -0.794 & 0.837 & -2.472 & 0.822 \\
$\phi$ & 5.457 & 0.353 & 4.785 & 6.177 \\
\hline
\end{tabular}

Tabela 3.6: Estatísticas das distribuições marginais a posteriori dos parâmetros para o modelo Tobit inflacionado.

\begin{tabular}{ccccc}
\hline Parâmetros & Média & D.P. & Q 2,5\% & Q 97,5\% \\
\hline$\beta_{0}$ & -1.561 & 0.119 & -1.807 & -1.339 \\
$\beta_{1}$ & 1.583 & 0.170 & 1.257 & 1.926 \\
$\rho_{0}$ & -4.036 & 4.816 & -12.66 & -1.978 \\
$\rho_{1}$ & 0.770 & 4.094 & -1.751 & 6.466 \\
$\gamma_{0}$ & -2.780 & 0.436 & -3.690 & -1.972 \\
$\gamma_{1}$ & -0.807 & 0.867 & -2.550 & 0.862 \\
$\sigma$ & 0.198 & 0.010 & 0.180 & 0.222 \\
\hline
\end{tabular}


Note pela Tabela 3.5 que os intervalos de credibilidade obtidos para $\beta_{0}$ e $\beta_{1}$ não contemplam seus valores verdadeiros no modelo BIZU. Além disso, $\rho_{1}$ e $\gamma_{1}$ não foram significativos neste mesmo modelo.

Pela Tabela 3.6 podemos perceber que no modelo Tobit as médias a posteriori de $\beta_{0}$ e $\beta_{1}$ estão mais próximas dos valores verdadeiros dos parâmetros, o que era esperado pela a origem dos dados simulados. No entanto, $\rho_{1}$ e $\gamma_{1}$ continuam não significativos e $\rho_{0}$ foi subestimado devido a possibilidade de os valores extremos serem interpretados tanto como censura ou inflação.

Apesar da diferença nas estimativas dos parâmetros, praticamente não se observa diferença ente os gráficos de resíduos dos dois modelos (vide Figuras 3.4 e 3.5).

Pela Figura 3.6 nota-se que o modelo BIZU destacou como ponto influente a observação $y_{262}=$ 0.0001 e a observação $y_{362}=0.010$ também se destacou. Ambas as observações correspondem a pontos próximos da borda do intervalo $(0,1)$, onde a densidade beta apresenta certa sensibilidade. Já o modelo Tobit inflacionado não teve problemas para incorporar estas observações no modelo. 

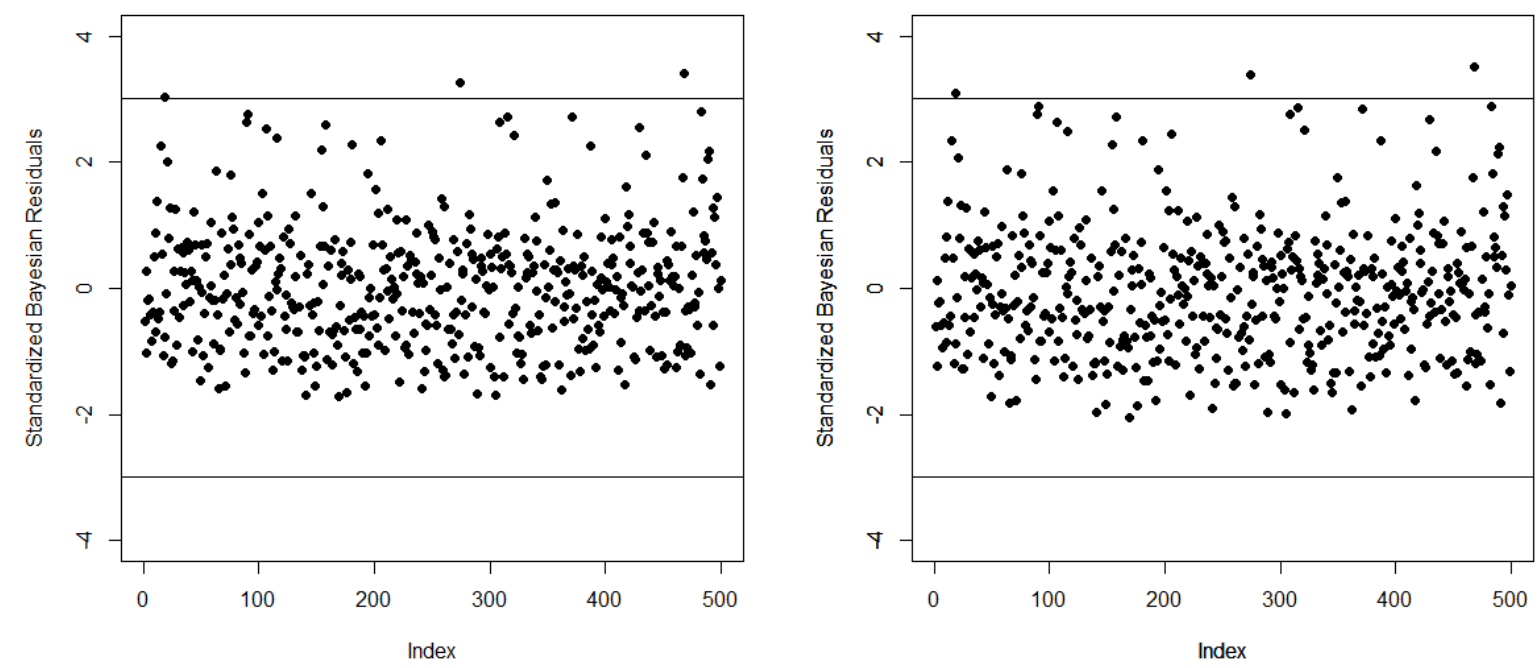

Figura 3.4: Gráfico dos resíduos Bayesianos padronizados pelas observações para os modelos BIZU (esquerda) e Tobit inflacionado (direita).
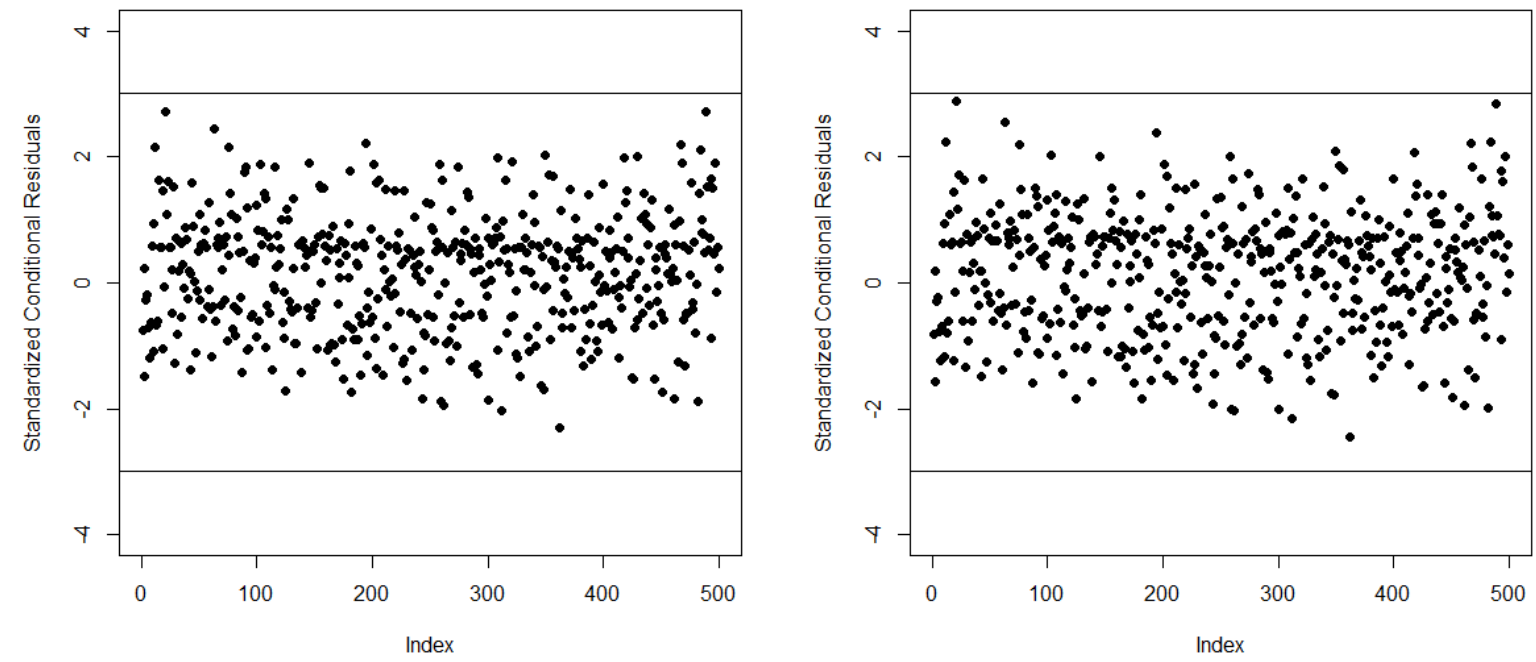

Figura 3.5: Gráfico dos resíduos Bayesianos condicionais padronizados pelas observações para os modelos BIZU (esquerda) e Tobit inflacionado (direita). 

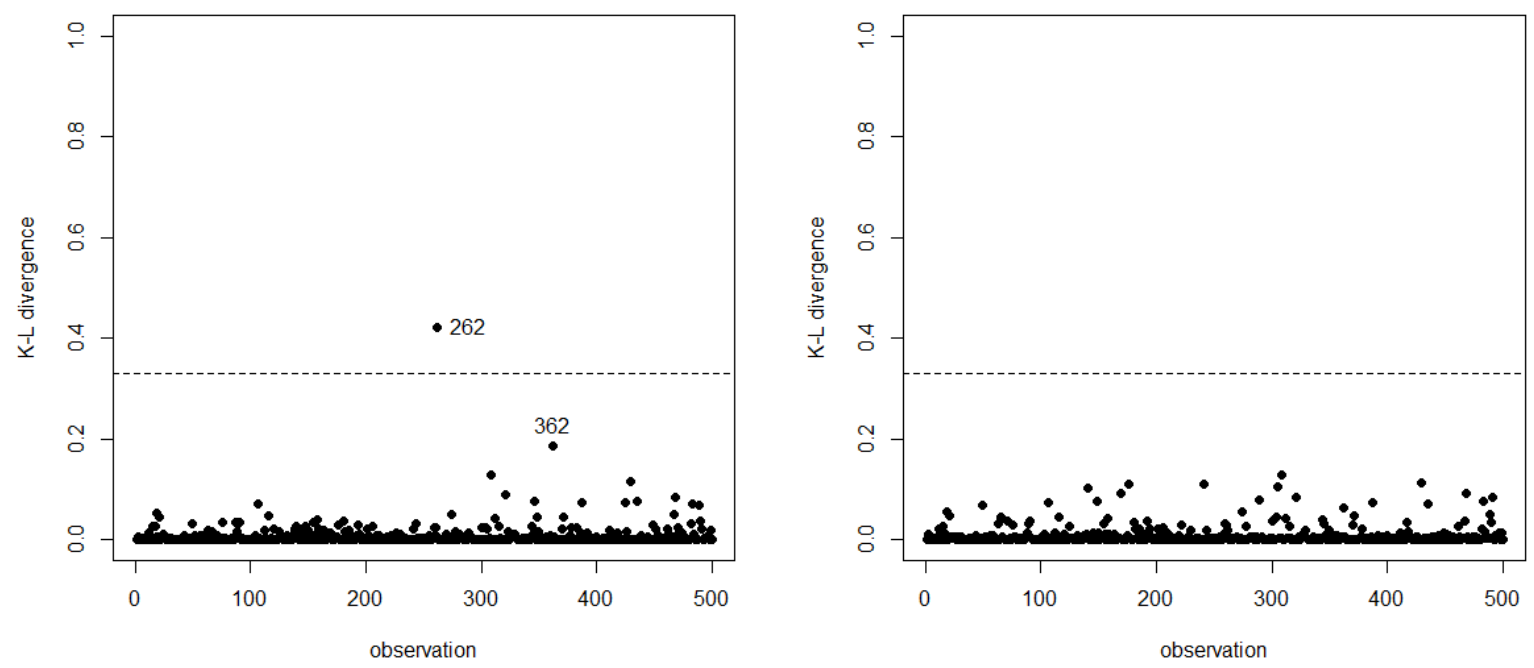

Figura 3.6: Gráfico da divergência de K-L para o modelo BIZU (esquerda) e Tobit inflacionado (direita). 


\subsubsection{Conclusão}

A partir deste estudo de simulação podemos notar a forte competitividade entre os modelos de regressão beta inflacionado e o modelo Tobit normal duplamente censurado e inflacionado para modelagem de dados contínuos em $[0,1]$, ressaltando que os modelos podem ser facilmente descolados para qualquer intervalo fechado $[a, b]$.

Podemos perceber uma certa vantagem do modelo beta inflacionado em relação a flexibilidade da distribuição beta. No entanto o modelo Tobit duplamente censurado conseguiu estimativas bem próximas dos valores verdadeiros dos parâmetros para os dados com distribuição beta. Há ainda a possibilidade de atribuir assimetria no modelo Tobit duplamente censurado utilizando o modelo Tobit normal potência.

O modelo Tobit mostrou-se superior ao modelo beta inflacionado na presença de dados próximos da borda $[0,1]$, como vimos anteriormente em (1), a distribuição beta possui restrições em sua densidade para os valores que se aproximam das extremidade do intervalo. Com isso, as observações próximas as extremidades apresentam influência acima da desejada na estimação dos parâmetros e em alguns cados podendo trazer problemas de identificabilidade na função de verossimilhança. Outra vantagem do modelo Tobit é a possibilidade de interpretação dos valores extremos tanto como inflação, como também podem ser interpretados como censura.

\subsection{Aplicações}

\subsubsection{Proporções de empregados do sexo feminino no setor de indústria}

A aplicação a seguir foi elaborada a partir dos dados da RAIS 2007 - MTE, obtidos em parceria com IPEA-DISET. Foi utilizada uma amostra aleatória de 700 empresas do setor de indústrias e foram utilizadas as seguintes variáveis:

- $\boldsymbol{y}=(\mathrm{N}$. de empregados do sexo feminino)/(N. total de empregados);

- $\boldsymbol{x}_{\mathbf{1}}=\log ($ Renda mensal média $)$;

- $\boldsymbol{x}_{\mathbf{2}}=$ Tempo de estudo médio;

- $\boldsymbol{v}_{\mathbf{1}}=$ Número de contratos;

- $\boldsymbol{v}_{\mathbf{2}}=$ Idade da empresa.

O modelo foi construído da seguinte forma:

$$
\begin{aligned}
\log \left(\frac{\mu_{i}}{1-\mu_{i}}\right) & =\beta_{0}+\beta_{1} x_{1 i}+\beta_{2} x_{2 i} \\
\log \left(\frac{\delta_{0 i}}{1-\delta_{0 i}-\delta_{1 i}}\right) & =\rho_{0}+\rho_{1} v_{1 i}+\rho_{2} v_{2 i} \\
\log \left(\frac{\delta_{1 i}}{1-\delta_{0 i}-\delta_{1 i}}\right) & =\gamma_{0}+\gamma_{1} v_{1 i}+\gamma_{2} v_{2 i} .
\end{aligned}
$$

A proporção de zeros na amostra foi de $30 \%$ e a proporção de uns foi de $8 \%$.

No procedimento de estimação via MCMC foi considerada apenas uma cadeia com um burn-in de tamanho 20.000 e em seguida gerada uma amostra de tamanho 50.000 com saltos de tamanho 40 . Foi também verificada a convergência da cadeia e baixo índice de autocorrelação. O procedimento foi realizado via OpenBugs. O custo computacional foi semelhante para os modelos BIZU e Tobit duplamente censurado e inflacionado, em torno de 40 minutos. Para o modelo Tobit normal-potência duplamente censurado e inflacionado o custo computacional foi quase o dobro. Foram atribuídas 
prioris $N\left(0,10^{3}\right)$ para os parâmetros ligados as covariáveis e para os parâmetros de precisão e assimetria foram atribuídos:

$$
\begin{array}{cl}
\phi \sim U(0,100), \quad & (\text { para o modelo BIZU) } . \\
(1 / \sigma) \sim \operatorname{Gama}(1,0.04), \quad \text { (para o modelo Tobit normal potência inflacionado) }, \\
\alpha \sim \operatorname{Gama}(0.1,0.1), \quad \text { (para o modelo Tobit normal potência inflacionado) },
\end{array}
$$

Pela Tabela 3.7 podemos observar que pelos quatro critérios o modelo BIZU foi superior ao modelo Tobit duplamente censurado e inflacionado.

Analisando a Tabela 3.8, o modelo mostra que a proporção de empregados do sexo feminino está inversamente relacionada com a renda mensal média e diretamente relacionada com o tempo de estudo médio. Este resultado nos dá um indicativo de que a mão de obra feminina no setor de indústria é em média mais qualificada e de remuneração inferior a mão de obra masculina. A presença de zeros e uns está inversamente relacionada ao número de empregados da empresa. Isto é, empresas com baixo número de empregados estão mais propensas a ter apenas empregados do mesmo sexo. E ainda, observou-se que empresas mais antigas estão mais propensas a ausência de empregados do sexo feminino. Pelas Tabelas 3.9 e 3.10, notamos que utilizando o Tobit normal inflacionado e Tobit normal potência inflacionado os modelos seguem praticamente a mesma interpretação, com a diferença de que o parâmetro $\rho_{2}$ não foi significativo.

A Figura 3.9 mostra que pela divergência K-L nenhuma observação se destacou como ponto influente em nenhum dos modelos. No entanto, nota-se que no modelo BIZU alguns pontos ficaram mais elevados do que no gráfico do modelo Tobit.

Pela distância- $\chi^{2}$, o modelo BIZU destacou como ponto influente a observação $y_{320}=0$, como podemos observar na Figura 3.10. Esta observação corresponde a uma empresa com 22 empregados, próximo do valor da média amostral que é de 26 empregados. Além disso é uma empresa antiga, com aproximadamente 46 anos de idade, e a idade média da amostra é de apenas 12, 7 anos.

Tabela 3.7: Comparação entre os modelos via DIC, $\widehat{E A I C}, \widehat{E B I C}$ e LMPL.

\begin{tabular}{lcccc}
\hline Modelo & DIC & $\widehat{E A I C}$ & $\widehat{E B I C}$ & LMPL \\
\hline BIZU & 774.56 & 784.63 & 830.15 & -387.66 \\
Tobit inf. & 808.50 & 818.68 & 864.193 & -404.96 \\
Tobit $\alpha$-potência & 809.47 & 821.11 & 871.17 & -406.03 \\
\hline
\end{tabular}


Tabela 3.8: Estatísticas das distribuições marginais a posteriori dos parâmetros para o modelo BIZU.

\begin{tabular}{ccccc}
\hline Parâmetros & Média & D.P. & Q 2,5\% & Q 97,5\% \\
\hline$\beta_{0}$ & 4.375 & 0.766 & 2.877 & 5.889 \\
$\beta_{1}$ & -0.929 & 0.123 & -1.1730 & -0.687 \\
$\beta_{2}$ & 0.140 & 0.028 & 0.085 & 0.195 \\
$\rho_{0}$ & 0.637 & 0.222 & 0.203 & 1.075 \\
$\rho_{1}$ & -0.264 & 0.027 & -0.320 & -0.212 \\
$\rho_{2}$ & 0.036 & 0.015 & 0.005 & 0.066 \\
$\gamma_{0}$ & 0.138 & 0.351 & -0.548 & 0.827 \\
$\gamma_{1}$ & -0.024 & 0.055 & -0.438 & -0.222 \\
$\gamma_{2}$ & -0.794 & 0.027 & -0.080 & 0.028 \\
$\phi$ & 3.323 & 0.204 & 2.937 & 3.739 \\
\hline
\end{tabular}

Tabela 3.9: Estatísticas das distribuições marginais a posteriori dos parâmetros para o modelo Tobit normal inflacionado.

\begin{tabular}{ccccc}
\hline Parâmetros & Média & D.P. & Q 2,5\% & Q 97,5\% \\
\hline$\beta_{0}$ & 7.598 & 1.456 & 4.910 & 10.60 \\
$\beta_{1}$ & -1.636 & 0.239 & -2.132 & -1.197 \\
$\beta_{2}$ & 0.274 & 0.046 & 0.185 & 0.369 \\
$\rho_{0}$ & 1.477 & 0.408 & 0.714 & 2.319 \\
$\rho_{1}$ & -0.638 & 0.114 & -0.894 & -0.448 \\
$\rho_{2}$ & 0.017 & 0.025 & -0.032 & 0.066 \\
$\gamma_{0}$ & 0.750 & 0.561 & -0.317 & 1.895 \\
$\gamma_{1}$ & -0.614 & 0.154 & -0.963 & -0.361 \\
$\gamma_{2}$ & -0.049 & 0.041 & -0.137 & 0.025 \\
$\sigma$ & 0.288 & 0.012 & 0.265 & 0.315 \\
\hline
\end{tabular}

Tabela 3.10: Estatisticas das distribuições marginais a posteriori dos parâmetros para o modelo Tobit normal potência inflacionado.

\begin{tabular}{ccccc}
\hline Parâmetros & Média & D.P. & Q 2,5\% & Q 97,5\% \\
\hline$\alpha$ & 1.532 & 0.426 & 0.682 & 2.340 \\
$\beta_{0}$ & 10.664 & 3.625 & 5.395 & 19.730 \\
$\beta_{1}$ & -2.232 & 0.639 & -3.767 & -1.282 \\
$\beta_{2}$ & 0.289 & 0.058 & 0.181 & 0.415 \\
$\rho_{0}$ & 1.455 & 0.392 & 0.736 & 2.278 \\
$\rho_{1}$ & -0.624 & 0.107 & -0.859 & -0.441 \\
$\rho_{2}$ & 0.019 & 0.024 & -0.029 & 0.066 \\
$\gamma_{0}$ & 0.708 & 0.545 & -0.314 & 1.819 \\
$\gamma_{1}$ & -0.612 & 0.152 & -0.966 & -0.363 \\
$\gamma_{2}$ & -0.046 & 0.041 & -0.136 & 0.026 \\
$\sigma$ & 0.319 & 0.028 & 0.253 & 0.364 \\
\hline
\end{tabular}



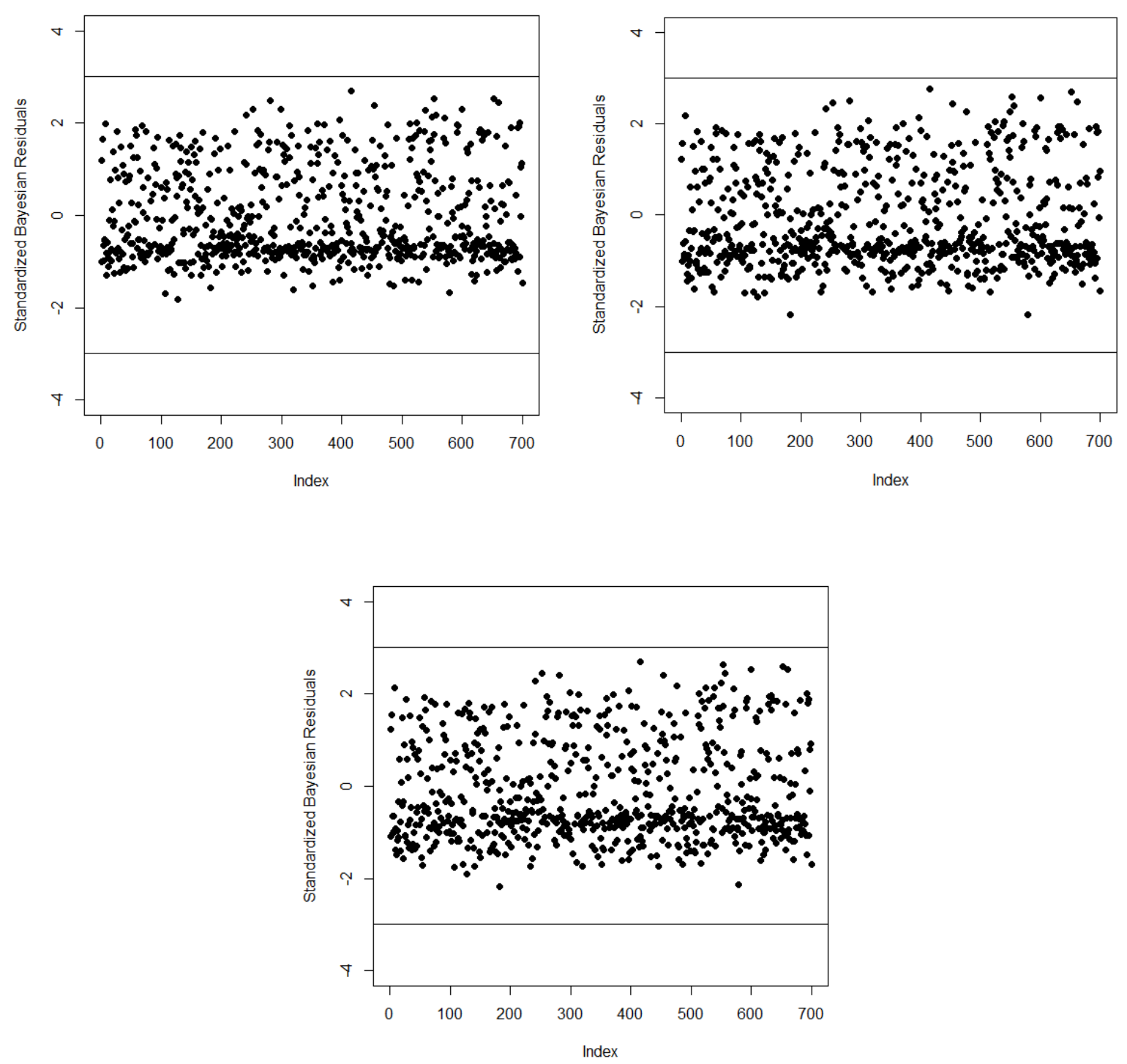

Figura 3.7: Gráfico dos resíduos Bayesianos padronizados pelas observações para os modelos BIZU (esquerda superior), Tobit normal inflacionado (direita superior) e Tobit normal potência inflacionado (inferior). 

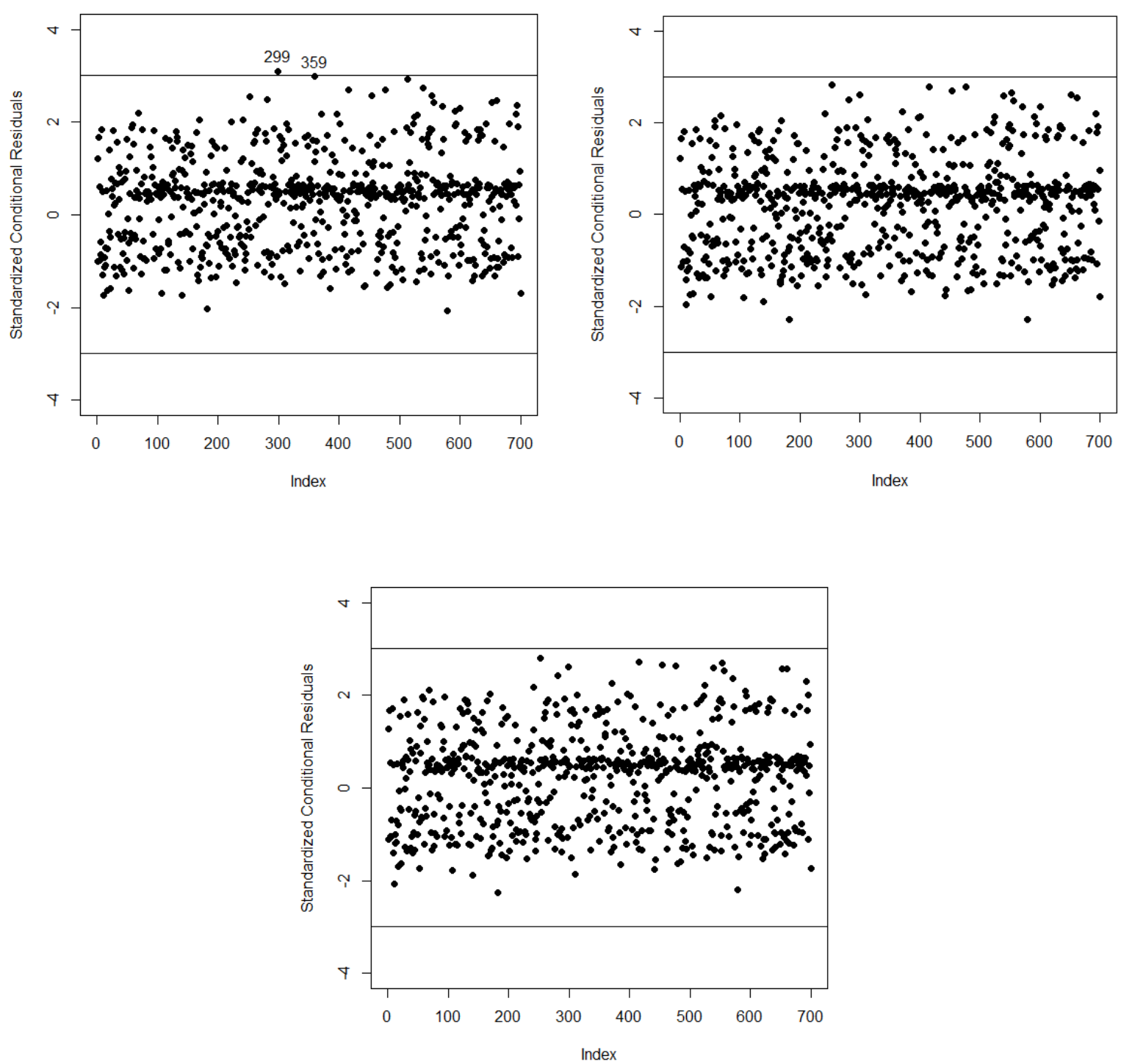

Figura 3.8: Gráfico dos resíduos Bayesianos condicionais padronizados pelas observações para os modelos BIZU (esquerda superior), Tobit normal inflacionado (direita superior) e Tobit normal potência inflacionado (inferior). 

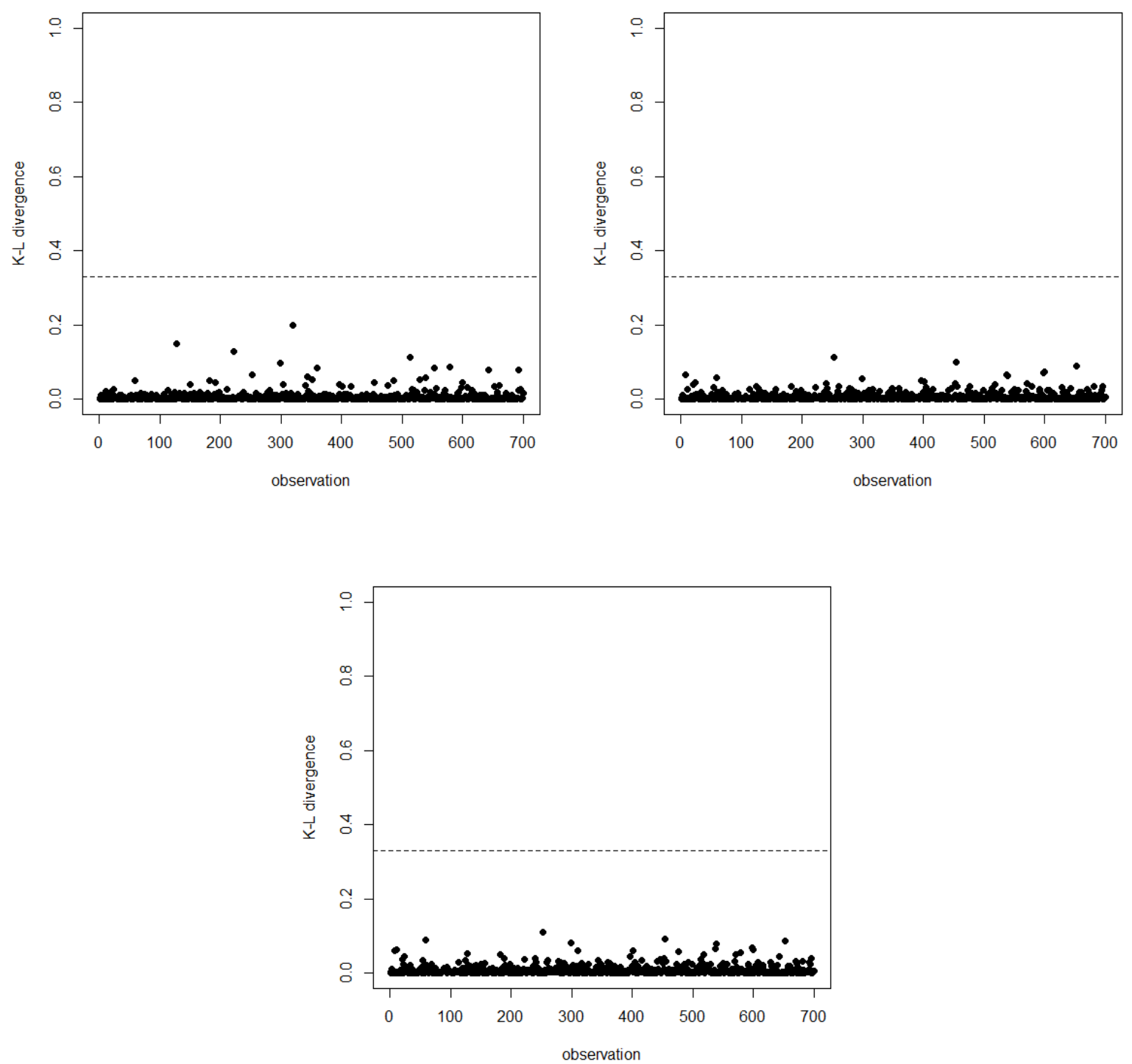

Figura 3.9: Gráfico da divergência de K-L para os modelos BIZU (esquerda superior), Tobit normal inflacionado (direita superior) e Tobit normal potência inflacionado (inferior). 

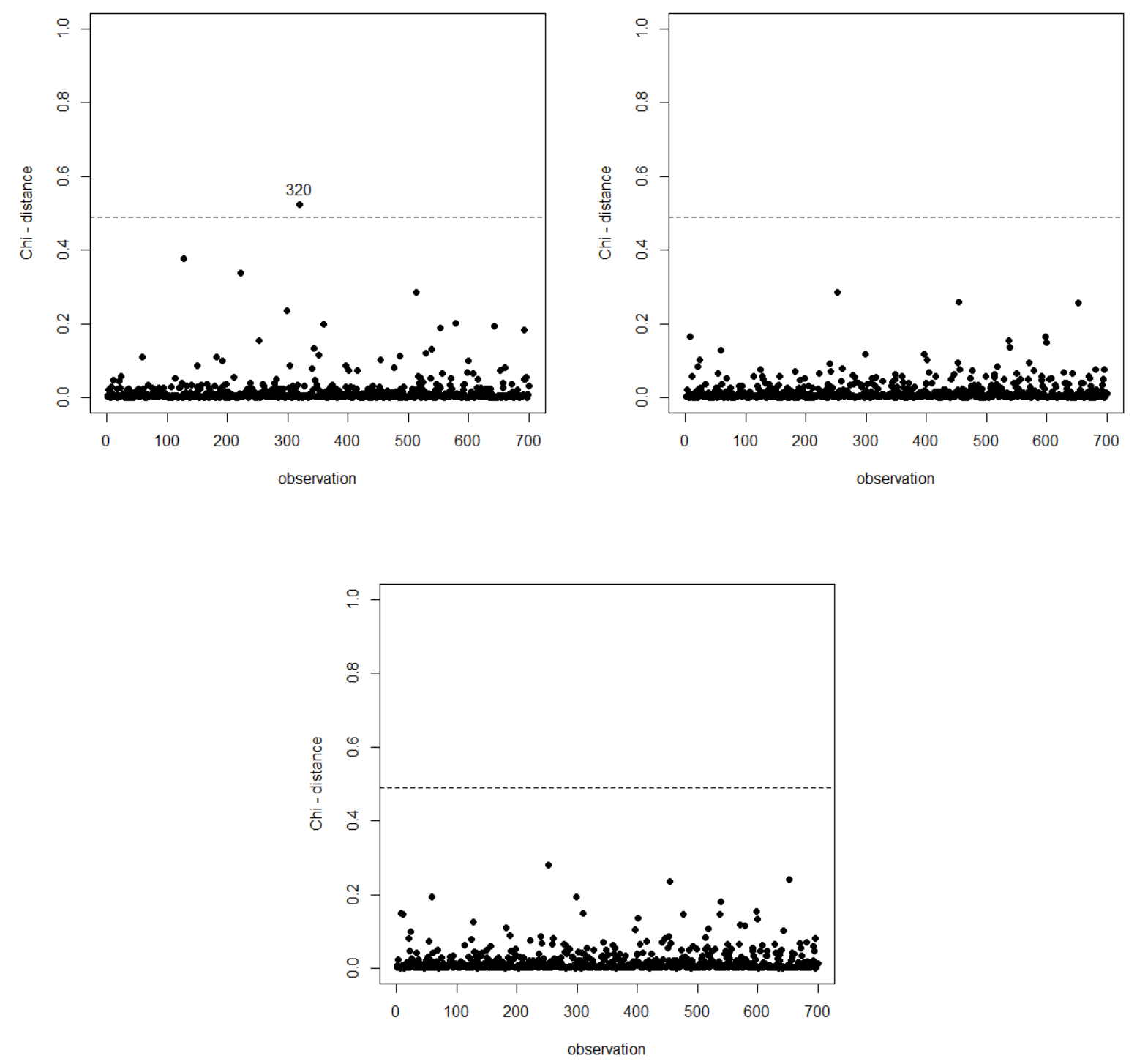

Figura 3.10: Gráfico da distância- $\chi^{2}$ para os modelos BIZU (esquerda superior), Tobit normal inflacionado (direita superior) e Tobit normal potência inflacionado (inferior). 


\subsubsection{Proporções de canais}

A aplicação a seguir foi elaborada a partir dos dados referentes à difusão de televisão a cabo nos EUA. As observações correspondem a 282 comunidades, que são essencialmente áreas de franquia individual com assinantes de TV a cabo. Os dados foram coletados pela Comissão Federal de Comunicações (FCC) dos EUA em conjunção a implementação da ata de competição e proteção ao consumidor de televisão a cabo de 1992. Para o estudo foram utilizadas as seguintes variáveis:

- $\boldsymbol{y}=$ proporção de assinantes de TV a cabo que adquirem serviços adicionais;

- $\boldsymbol{x}_{\mathbf{1}}=\log$ (Renda média da franquia);

- $\boldsymbol{v}_{\mathbf{1}}=$ Número de canais com sinal local na cidade;

O modelo foi construído da seguinte forma:

$$
\begin{aligned}
\log \left(\frac{\mu_{i}}{1-\mu_{i}}\right) & =\beta_{0}+\beta_{1} x_{1 i} \\
\log \left(\frac{\delta_{0 i}}{1-\delta_{0 i}}\right) & =\rho_{0}+\rho_{1} v_{1 i}
\end{aligned}
$$

A proporção de zeros na amostra foi de $20 \%$.

No procedimento de estimação via MCMC foi considerada uma cadeia com um burn-in de tamanho 40.000 e em seguida gerada uma amostra de tamanho 5.000 com saltos de tamanho 40 . Foi também verificada a convergência da cadeia e baixo índice de autocorrelação. O procedimento foi realizado via OpenBugs. O custo computacional foi semelhante para os modelos BIZU e Tobit duplamente censurado e inflacionado, em torno de 30 minutos. Para o modelo Tobit normal-potência duplamente censurado e inflacionado o custo computacional foi quase o dobro.

Conforme a robustez da escolha das prioris observada nos estudos de simulação, foram atribuídas prioris pouco informativas para os parâmetros ligados as covariáveis, da forma: $N\left(0,10^{3}\right)$. Para os parâmetros de precisão e assimetria foram utilizadas:

$$
\begin{aligned}
& 1 / \sigma \sim \operatorname{Gama}(1.1,0.04), \quad \text { (para os modelos tobit inflacionado) } \\
& \phi \sim \operatorname{Gama}(1,0.033), \quad \text { (para o modelo BIZ). } \\
& \alpha \sim \operatorname{Gama}(0.1,0.1), \quad \text { (para o modelo tobit normal potência) }
\end{aligned}
$$


Tabela 3.11: Comparação entre os modelos via DIC, $\widehat{E A I C}, \widehat{E B I C}$ e LMPL.

\begin{tabular}{l|cccc}
\hline Modelo & DIC & $\widehat{E A I C}$ & $\widehat{E B I C}$ & LMPL \\
\hline BIZU & 121.91 & 126.93 & 145.14 & -61.59 \\
Tobit inf. & 114.63 & 125.82 & 144.02 & -61.04 \\
Tobit NP & 109.03 & 117.43 & 142.93 & -56.11 \\
\hline
\end{tabular}

Note que pelos quatro critérios o modelo BIZ foi inferior aos modelos Tobit duplamente censurado e inflacionado.

Tabela 3.12: Estatísticas das distribuições marginais a posteriori dos parâmetros para o modelo BIZ.

\begin{tabular}{c|c|c|c|c}
\hline Parâmetros & Média & D.P. & Q 2,5\% & Q 97,5\% \\
\hline$\beta_{0}$ & -8.29 & 1.52 & -11.29 & -5.37 \\
$\beta_{1}$ & 0.73 & 0.14 & 0.44 & 1.03 \\
$\rho_{0}$ & -0.70 & 0.31 & -1.34 & -0.09 \\
$\rho_{1}$ & -0.09 & 0.04 & -0.19 & -0.001 \\
$\phi$ & 5.95 & 0.53 & 4.95 & 7.05 \\
\hline
\end{tabular}

Tabela 3.13: Estatísticas das distribuições marginais a posteriori dos parâmetros para o modelo Tobit normal inflacionado de zero.

\begin{tabular}{c|c|c|c|c}
\hline Parâmetros & Média & D.P. & Q 2,5\% & Q 97,5\% \\
\hline$\beta_{0}$ & -13.09 & 2.68 & -18.65 & -8.11 \\
$\beta_{1}$ & 1.16 & 0.25 & 0.68 & 1.69 \\
$\rho_{0}$ & -5.63 & 5.76 & -20.94 & -0.33 \\
$\rho_{1}$ & -5.71 & 6.31 & -21.29 & 0.13 \\
$\sigma$ & 0.22 & 0.02 & 0.18 & 0.26 \\
\hline
\end{tabular}

Tabela 3.14: Estatísticas das distribuições marginais a posteriori dos parâmetros para o modelo tobit normal-potência inflacionado de zero.

\begin{tabular}{c|c|c|c|c}
\hline Parâmetros & Média & D.P. & Q 2,5\% & Q 97,5\% \\
\hline$\alpha$ & 2.19 & 0.66 & 0.82 & 3.43 \\
$\beta_{0}$ & -18.74 & 4.88 & -28.70 & -9.74 \\
$\beta_{1}$ & 1.64 & 0.43 & 0.84 & 2.54 \\
$\rho_{0}$ & 1.25 & 0.58 & -2.64 & -0.35 \\
$\rho_{1}$ & -0.08 & 0.07 & -0.25 & 0.05 \\
$\sigma$ & 0.22 & 0.01 & 0.18 & 0.26 \\
\hline
\end{tabular}

Note que pelos modelos Tobit normal e Tobit normal-potência o parâmetro $\rho_{1}$ não foi significativo, indicando que o número de canais com sinal local na cidade não tem influência determinante na probabilidade de haver assinatura de TV a cabo na comunidade. Segundo o modelo BIZU esta relação foi significativa, indicando que quanto maior o número de canais locais com sinal, maior a probabilidade de haver assinatura de TV a cabo (menor probabilidade de não haver assinaturas na comunidade). Em relação a renda média da franquia, foi observado pelos três modelos que a renda média possui relação direta com o aumento da proporção de assinaturas. 

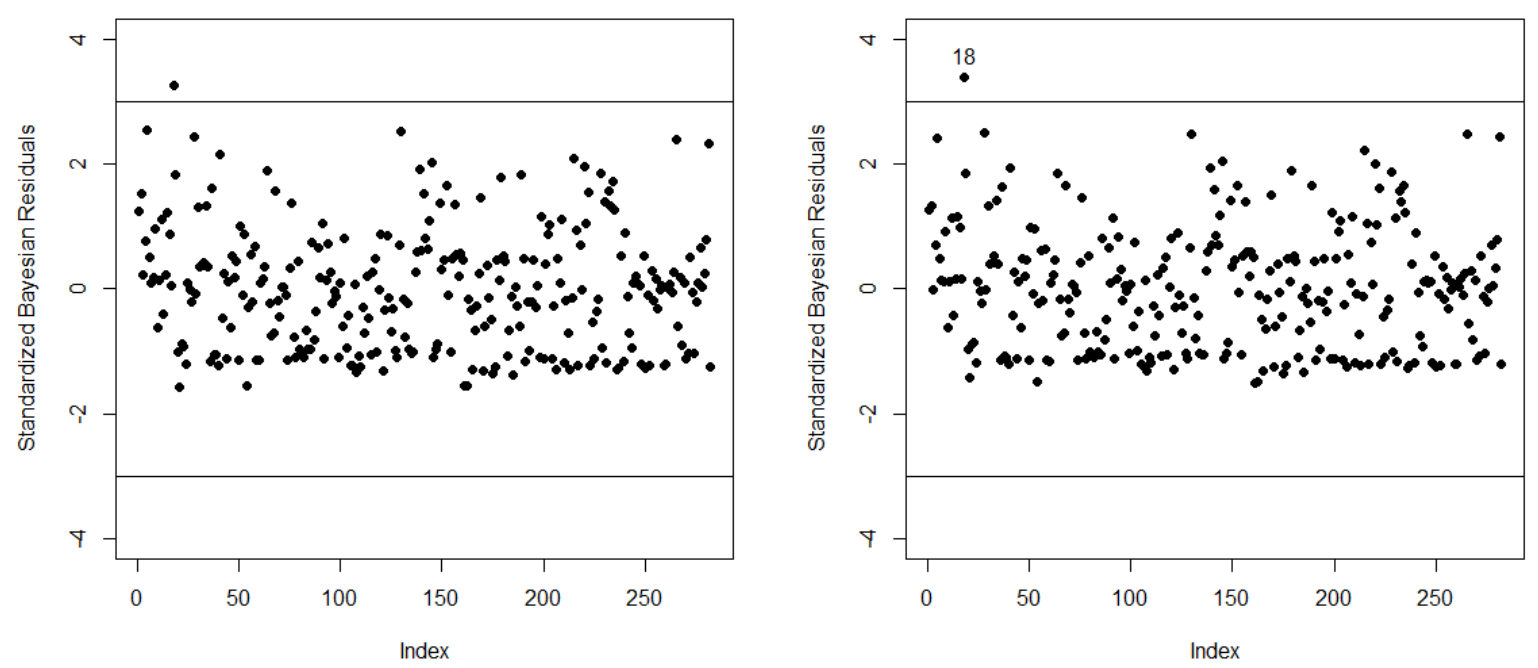

Figura 3.11: Gráfico dos resíduos Bayesianos padronizados pelas observações para os modelos BIZ (esquerda) e Tobit inflacionado (direita).

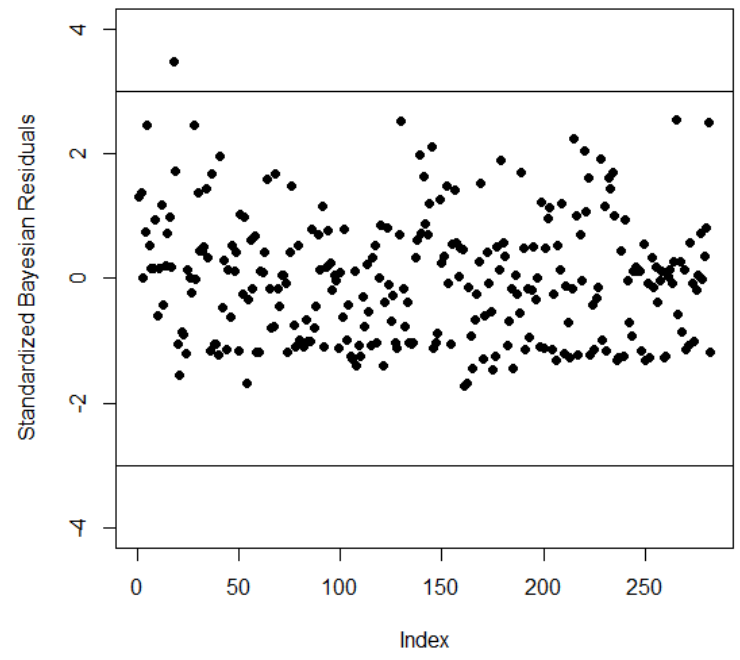

Figura 3.12: Gráfico dos resíduos Bayesianos padronizados pelas observações para o modelo tobit normalpotência inflacionado. 

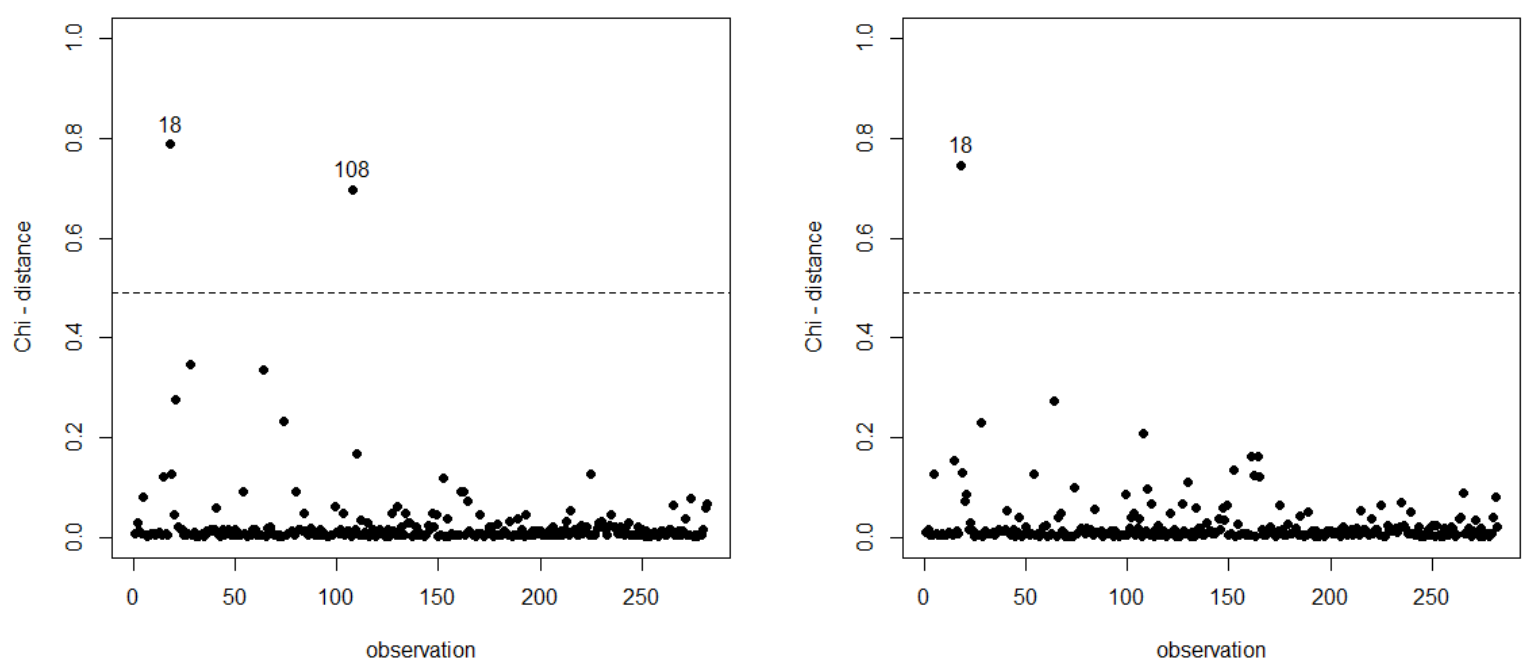

Figura 3.13: Gráfico da distância- $\chi^{2}$ para o modelo BIZ (esquerda) e tobit inflacionado (direita).

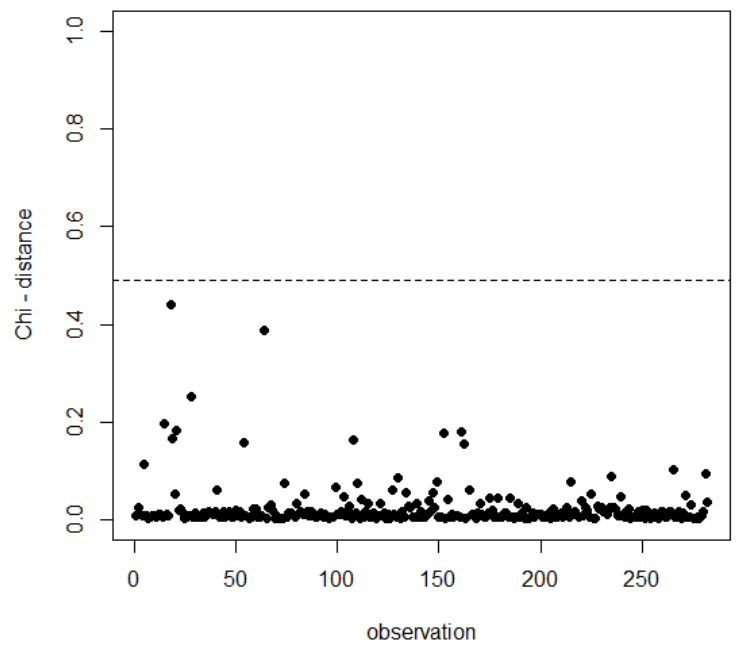

Figura 3.14: Gráfico da distância- $\chi^{2}$ para o modelo tobit normal-potência.

Pela distância- $\chi^{2}$ apresentada na Figura 3.13, o modelo BIZ destacou como ponto influente a observação $y_{108}=0,007$. Esta observação está bem próxima da borda inferior do intervalo $(0,1)$. Em ambos os modelos destacaram a observação $y_{18}$ que corresponde a observação máxima da amostra. Pela Figura 3.14, nota-se que no modelo tobit normal-potência não foi destacado nenhum ponto influente. 


\section{Capítulo 4}

\section{Modelos Dinâmicos}

Nesta seção iremos nos concentrar na classe de modelos dinâmicos Bayesianos para séries temporais de taxas ou proporções contínuas onde os valores extremos podem ser incorporados na modelagem. O modelo dinâmico beta foi apresentado da-Silva et al. (2011) para modelagem de dados de proporções contínuas em séries de tempo onde não temos a presença dos valores extremos do intervalo $[0,1]$, uma versão hierárquica do modelo dinâmico beta foi apresentada por da-Silva e Migon (2012) onde foi mantida esta mesma limitação ao conjuntos de dados.

Nosso objetivo é propor o modelo dinâmico beta inflacionado, utilizando a distribuição $B I Z U$ proposta por Ospina (2008) e uma outra abordagem utilizando o modelo dinâmico Tobit duplamente censurado, estendendo a idéia proposta por Tobin (1958) e o modelo de Moulton e Halsey (1995), com uma abordagem diferente da proposta por Wei (1999), utilizando censura em ambos os lados, para adequá-lo ao contexto de dados de proporções.

Inicialmente introduziremos a abordagem Bayesiana de modelos dinâmicos lineares. Em seguida iremos propor a formulação do modelo dinâmico beta inflacionado e uma proposta para o modelo dinâmico Tobit duplamente censurado.

\subsection{Abordagem Bayesiana em Modelos Dinâmicos}

A abordagem Bayesiana dos modelos dinâmicos possui a interessante capacidade de incorporar, no modelo, todas as informações relevantes disponíveis: desde dados históricos, experiências concretas ou subjetivas, assim como conhecimento de fenômenos futuros. Além de previsões rotineiras, exceções podem também ser implementadas por antecipações ou em bases retrospectivas. Algumas vantagens, em relação à análise frequentista, são obtidas naturalmente, como intervenção, função de transferência e análise retrospectiva (alisamento), entre outras.

O modelo completo pode ser decomposto em componentes lineares dinâmicas independentes (MLD) onde cada uma descreve aspectos particulares do estudo. Esta estrutura de modelo permite que tanto análises prospectivas como retrospectivas sejam acomodadas facilmente.

A inferência é feita de maneira sequencial, ou seja, é refeita a cada tempo $t$ para o conjunto de informações $Y_{t}$. O processo inicia-se baseado no conjunto de informações disponível antes de se observar os dados, ou seja, no tempo $t=0$. Este conjunto de dados (subjetivos ou não) é denotado por $D_{0}$. Toda a informação sobre o futuro $(t>0)$ será condicional a $D_{0}$. Ao chegar no tempo $t$, a informação disponível está concentrada em $D_{t}=\left\{y_{t}, D_{t-1}\right\}$. Assim, quando o tempo evolui de $t$ para $t+1, y_{t+1}$ é inserido no conjunto de informações, portanto $D_{t+1}=\left\{y_{t+1}, D_{t}\right\}$. Há, também, a possibilidade de inserir informações além daquela obtida com as observações, de modo que $D_{t+1}$ pode ser generalizado como $D_{t+1}=\left\{I_{t+1}, D_{t}\right\}$, onde $I_{t+1}$ corresponde a toda informação adicional obtida. No caso em que toda informação adicional obtida em cada tempo $t$ é a própria observação $y_{t}$, então $D_{t}=\left\{D_{0}, y_{1}, \ldots, y_{n}\right\}$, e é denominado um sistema fechado. Sistemas que admitem a entrada de informações além das observações da série é chamado de sistema aberto. Para estudos de previsão, o interesse está em obter as distribuições preditivas $\left(Y_{t+h} \mid D_{t}\right), h>0$.

A modelagem dinâmica parte da idéia de que as observações flutuam em torno de uma média, 
denominada nível. Esta média, no entanto, está sujeita a variações ao longo do tempo. Estas variações com o tempo são, essencialmente, estocásticas e vão depender de erros, ou perturbações, da evolução. Sendo assim, a evolução é modelada através de um passeio aleatório e a novidade desta abordagem está na evolução paramétrica caracterizada pela equação que relaciona sucessivos valores dos parâmetros, denominada Equação de evolução. A forma geral dos modelos dinâmicos lineares apresentam a seguinte estrutura (West e Harrison, 1997; pg. 102):

- Equação de observação:

$$
y_{t}=F_{t}^{\prime} \theta_{t}+\epsilon_{t}
$$

Em geral, assume-se que $\epsilon_{t} \sim N\left(0, V_{t}\right)$.

- Equação de evolução ou Sistema de Equações:

$$
\theta_{t}=G_{t} \theta_{t-1}+w_{t}
$$

Em geral, assume-se que $\omega_{t} \sim N\left(0, W_{t}\right)$.

Esta estrutura parte da idéia de que a série de observações $y_{t}$ são independentes condicionalmente a $\theta_{t}$ e $V_{t}$, onde $F_{t}^{\prime} \theta_{t}=E\left(Y_{t} \mid \theta_{t}\right)$ e $V_{t}=V\left(Y_{t} \mid \theta_{t}\right)$. $F_{t}$ é um vetor de constantes conhecidas, podendo ser um conjunto de variáveis explicativas. $\theta_{t}$ corresponde a um vetor de parâmetros. Na abordagem Bayesiana, $\theta_{t}$ é interpretado como um vetor de variáveis aleatórias onde $E\left(\theta_{t} \mid D_{t}\right)=m_{t}$ e $V\left(\theta_{t} \mid D_{t}\right)=$ $W_{t}$. $G_{t}$ corresponde à matriz de termos conhecidos que define a evolução sistemática dos parâmetros. Em geral, assume-se que $\epsilon_{t}$ e $\omega_{t}$ são mutuamente independentes. O sistema de equações é iniciado assumindo que $\theta_{0}$ possui uma distribuição a priori conhecida.

O modelo linear dinâmico é caracterizado pelo conjunto

$$
\left(F_{t}, G_{t}, V_{t}, W_{t}\right)
$$

Partindo desta estrutura, pode-se caracterizar os seguintes casos:

- Modelos clássicos de séries temporais com: $F_{t}=F$ e $G_{t}=G, \forall t$.

- Modelos clássicos de regressão linear com: $G_{t}=I_{p}$ e $V_{t}=0, \forall t$.

O processo inferencial é composto, a cada passo no tempo, pelos processos de evolução, previsão e atualização, organizados da seguinte forma:

$$
\begin{array}{lll}
\mu_{t-1} \mid D_{t-1} \stackrel{\text { EVOLUCAO }}{\longrightarrow} & \mu_{t}\left|D_{t-1} \stackrel{\text { ATUALIZACAO }}{\rightarrow} \mu_{t}\right| D_{t} \\
\text { post. } & \text { priori } & \text { post. } \\
& \downarrow & \\
& Y_{t} \mid D_{t-1} & \\
& \text { previsao } &
\end{array}
$$

Figura 4.1: Estrutura do modelo linear dinâmico

\subsection{Modelo dinâmico beta inflacionado}

Considere $y_{1}, \ldots, y_{T}$ uma série temporal onde $Y_{t} \sim B I Z U\left(\alpha, \gamma, \mu_{t}, \phi_{t}\right)(2.2)$, onde $0<\gamma, \alpha, \mu_{t}<$ 1 e $\phi_{t}>0$ para $t=1, \ldots, T$.

Segue abaixo a estrutura do modelo dinâmico beta inflacionado: 
- Equação das observações:

$$
\begin{aligned}
& p\left(y_{t} \mid \alpha, \gamma, \mu_{t}, \phi_{t}\right)=B I Z U\left(y_{t} ; \alpha, \gamma, \mu_{t}, \phi_{t}\right)=\alpha^{\mathbb{1}_{\{0,1\}}}(1-\alpha)^{\mathbb{1}_{\{0,1\}}} \\
& \times \gamma^{\mathbb{1}_{\{1\}}}(1-\gamma)^{\mathbb{1}_{\{0,1\}}-\mathbb{1}_{\{1\}}} \\
& \times \frac{\Gamma\left(\phi_{t}\right)}{\Gamma\left(\mu_{t} \phi_{t}\right) \Gamma\left(\left(1-\mu_{t}\right) \phi_{t}\right)} y_{t}^{\mu_{t} \phi_{t}-1}\left(1-y_{t}\right)^{\left(1-\mu_{t}\right) \phi_{t}-1},
\end{aligned}
$$

- Transformações nos parâmetros com dinâmica:

$$
\eta_{1 t}=h_{1}\left(\mu_{t}\right) ; \quad \eta_{2 t}=h_{2}\left(\phi_{t}\right)
$$

Podemos considerar as opções das funções de ligação logito e log:

$$
h_{1}\left(\mu_{t}\right)=\log \left(\frac{\mu_{t}}{1-\mu_{t}}\right) \quad h_{2}\left(\phi_{t}\right)=\log \left(\phi_{t}\right)
$$

\section{- Equação estrutural:}

$$
\boldsymbol{\eta}_{t}=\boldsymbol{F}_{t} \boldsymbol{\theta}_{t}
$$

- Equação do sistema:

$$
\boldsymbol{\theta}_{t}=\boldsymbol{H}_{t} \boldsymbol{\theta}_{t-1}+\boldsymbol{w}_{t} ; \quad \boldsymbol{w}_{t} \sim N(0, \boldsymbol{W})
$$

\section{- Informação Inicial:}

$$
\left(\boldsymbol{\theta}_{0} \mid D_{0}\right) \sim N\left(\boldsymbol{m}_{0}, \boldsymbol{C}_{0}\right)
$$

Em resumo, estamos trabalhando com as seguintes notações

- $\boldsymbol{\theta}_{t}$ é o vetor s-dimensional de estados latentes;

- $\boldsymbol{\eta}_{t}=\left(\eta_{1 t}, \eta_{2 t}\right)^{\prime}$ é vetor $2 \times 1$ de parâmetros estruturais;

- $\boldsymbol{F}_{t}=\left(\boldsymbol{F}_{1 t}^{\prime}, \boldsymbol{F}_{2 t}^{\prime}\right)^{\prime}$ é matriz $2 \times s$;

- $\boldsymbol{H}_{t}$ é matriz $s \times s$ que especifica os efeitos nos estados latentes;

- $\boldsymbol{w}_{t}$ é o vetor de erros da equação do sistema;

- $\boldsymbol{W}$ é matriz $s \times s$ bloco-diagonal de covariância de $\boldsymbol{w}_{t}$;

- $\boldsymbol{\theta}=\left(\boldsymbol{\theta}_{0}, \ldots, \boldsymbol{\theta}_{T}\right) ; \boldsymbol{\eta}=\left(\boldsymbol{\eta}_{0}, \ldots, \boldsymbol{\eta}_{T}\right) ; \boldsymbol{y}=\left(y_{1}, \ldots, y_{T}\right)$.

\subsubsection{Análise Bayesiana}

Trabalhando com matriz de precisão: $\boldsymbol{\Phi}_{1}=\boldsymbol{W}^{-1}$ onde $\boldsymbol{\Phi}_{1}$ é bloco-diagonal $s \times s$. Suponha que $\boldsymbol{\Phi}_{1}$ siga distribuição Wishart, tal que $\boldsymbol{\Phi}_{1} \sim W\left(\nu_{1}, S_{1}\right)$. A distribuição a posteriori é dada por

$$
\begin{aligned}
p\left(\boldsymbol{\eta}, \boldsymbol{\theta}, \alpha, \gamma, \boldsymbol{\Phi}_{1} \mid \boldsymbol{y}\right) \propto & {\left[\prod_{t=1}^{T} B I Z U\left(y_{t} ; \alpha, \gamma, \boldsymbol{\eta}_{t}\right) N\left(\boldsymbol{\theta}_{t}, \boldsymbol{H}_{t} \boldsymbol{\theta}_{t-1} \boldsymbol{\Phi}_{1}^{-1}\right)\right] } \\
& \times \operatorname{Beta}\left(a_{\alpha}, b_{\alpha}\right) N\left(\boldsymbol{\theta}_{0}, \boldsymbol{m}_{0}, \boldsymbol{C}_{0}\right) W\left(\boldsymbol{\Phi}_{1}, \nu_{1}, S_{1}\right)
\end{aligned}
$$

Em geral, estamos interessados em trabalhar com as seguintes distribuições condicionais completas a posteriori $p\left(\boldsymbol{\theta} \mid \alpha, \gamma, \mathbf{\Phi}_{1}, \boldsymbol{y}\right), p\left(\boldsymbol{\Phi}_{1} \mid \boldsymbol{\theta}, \alpha, \gamma, \boldsymbol{y}\right), p\left(\alpha \mid \boldsymbol{\theta}, \gamma, \mathbf{\Phi}_{1}, \boldsymbol{y}\right)$ e $p\left(\gamma \mid \boldsymbol{\theta}, \alpha, \boldsymbol{\Phi}_{1}, \boldsymbol{y}\right)$. 
As duas primeiras distribuições são descritas de forma similar a apresentada por da-Silva e Migon (2012). Ou seja, $p\left(\boldsymbol{\theta} \mid \alpha, \gamma, \boldsymbol{\Phi}_{1}, \boldsymbol{y}\right)$ é obtido via FFBS. Assim o filtro pode ser desenvolvido para obter a posteriori condicional completa $p\left(\boldsymbol{\theta} \mid \alpha, \gamma, \boldsymbol{\Phi}_{1}, \boldsymbol{y}\right)$. Para a variância $W$, temos

$$
p\left(\boldsymbol{\psi}_{1} \mid \boldsymbol{\theta}, \alpha, \gamma, \boldsymbol{y}\right) \propto\left|\boldsymbol{\psi}_{1}\right|^{T / 2+\nu_{1}-(s+1) / 2} \exp \left\{-\operatorname{tr}\left(S S_{\theta} / 2+\boldsymbol{S}_{1}\right) \boldsymbol{\psi}_{1}\right\},
$$

$\operatorname{com} S S_{\theta_{t}}=\left(\boldsymbol{\theta}_{t}-\boldsymbol{G}_{t} \boldsymbol{\theta}_{t-1}\right)\left(\boldsymbol{\theta}_{t}-\boldsymbol{G}_{t} \boldsymbol{\theta}_{t-1}\right)^{\prime}$ e $S S_{\theta}=\sum_{t=1}^{T} S S_{\theta_{t}}$. Então $\left(\boldsymbol{\psi}_{1} \mid \boldsymbol{\theta}, \alpha, \gamma, \boldsymbol{y}\right) \sim W(T / 2+$ $\left.\nu_{1}, S S_{\theta}+\boldsymbol{S}_{1}\right)$. Além disso,

$$
\begin{aligned}
\left(\alpha \mid \boldsymbol{\theta}, \gamma, \boldsymbol{\Phi}_{1}, \boldsymbol{y}\right) & \sim \operatorname{Beta}\left(a_{\alpha}+\sum_{t=1}^{T} \mathbb{1}_{\{0,1\}}\left(y_{t}\right) ; b_{\alpha}+T-\sum_{t=1}^{T} \mathbb{1}_{\{0,1\}}\left(y_{t}\right)\right) \\
\left(\gamma \mid \boldsymbol{\theta}, \alpha, \boldsymbol{\Phi}_{1}, \boldsymbol{y}\right) & \sim \operatorname{Beta}\left(a_{\gamma}+\sum_{t=1}^{T} \mathbb{1}_{\{1\}}\left(y_{t}\right) ; b_{\gamma}+\sum_{t=1}^{T} \mathbb{1}_{\{0,1\}}\left(y_{t}\right)-\sum_{t=1}^{T} \mathbb{1}_{\{1\}}\left(y_{t}\right)\right) .
\end{aligned}
$$

\section{Utilizando o parâmetro de dispersão estático}

Seja o caso de $\zeta_{t}=\phi>0$, fixo para $t=1, \ldots, T$. Desta forma, $\eta_{t}=\eta_{1 t}$, isto é, nos parâmetros estruturais serão incluídos apenas $\eta_{1 t}$ e o parâmetro $\phi$ passa a ter um papel semelhante ao dos parâmetros $\alpha, \gamma$ e $\boldsymbol{\Phi}_{1}$ no modelo dinâmico, ou seja, $\phi$ não está relacionado ao processo dinâmico latente descrito na equação do sistema. Portanto, todos os desenvolvimentos anteriores são mantidos da mesma forma, com suas pequenas alterações devidas à mudança de dimensionalidade de $\eta_{t}$.

A distribuição condicional completa de $\phi$, expresso na escala de $\eta_{2}=h_{2}(\phi)$ é dada por:

$$
p\left(\eta_{2} \mid \boldsymbol{\theta}, \boldsymbol{\Phi}_{1}\right) \propto\left[\prod_{t=1}^{T} B I Z U\left(y_{t}, \alpha, \gamma\right)\right] p\left(\eta_{2}\right) .
$$

Tome $\eta_{2} \sim N\left(a_{\phi}, b_{\phi}\right)$.

\section{Distribuição preditiva}

Para previsões com o modelo dinâmico beta inflacionado, estamo interessado em obter a distribuição preditiva marginal dada por

$$
p\left(y_{t} \mid D_{t-1}\right)=\iiint p\left(y_{t} \mid \boldsymbol{\theta}_{t}, \alpha, \gamma, D_{t-1}\right) p\left(\boldsymbol{\theta}_{t} \mid D_{t-1}\right) p\left(\alpha \mid D_{t-1}\right) p\left(\gamma \mid D_{t-1}\right) d \boldsymbol{\theta}_{t} d \alpha d \gamma .
$$

No entanto, como a distribuição conjunta $p\left(y_{t}, \boldsymbol{\theta}_{t}, \alpha, \gamma \mid D_{t-1}\right)$ não pode ser expressa de forma fechada, a integração múltipla acima não pode ser resolvida analiticamente. No caso mais geral, de previsão a $k$ passos a frente, $k>0$, temos que seguir o seguinte procedimento recursivo:

1. A distribuição preditiva de $k$-passos a frente dos espaços de estados é

$$
p\left(\theta_{t+k}, D_{t}\right)=\int p\left(\theta_{t+k} \mid \theta_{t+k-1}\right) p\left(\theta_{t+k-1} \mid D_{t}\right) d \theta_{t+k-1} .
$$

2. A distribuição preditiva de $k$-passos a frente das observações é

$$
p\left(y_{t+k}, D_{t}\right)=\int p\left(y_{t+k} \mid \theta_{t+k}\right) p\left(\theta_{t+k} \mid D_{t}\right) d \theta_{t+k} .
$$

A prova pode ser verificada em (Petris et al., 2009, Cap. 2, pag. 70). Levando em conta todos os demais parâmetros estáticos envolvidos, a integração se torna ainda mais complexa. Com isso, faz-se necessário o uso de técnicas de aproximação como quadratura ou Monte Carlo. As técnicas 
de quadratura, como utilizadas em da-Silva et al. (2011), ficam bastante complicadas devido a dimensão dos parâmetros e a quantidade de integrais. A integração por meio da metodologia MCMC parecem mais viáveis a um primeiro momento, no entanto elevariam bastante o custo computacional do modelo. Uma alternativa mais adequada para este contexto é o uso da metodologia de filtro de partículas apresentada por Pitt e Shephard (1999). Na seção 4.2.2 é apresentado uma primeira tentativa de estimação em um estudo de simulação via MCMC para o modelo dinâmico BIZU e no capítulo 5 é detalhado o procedimento de estimação de modelos dinâmicos via filtro de partículas. Esta metodologia se apresentou mais adequada para obtenção da distribuição preditiva do modelo.

\subsubsection{Primeiro resultado com simulações via MCMC}

Apresentamos a seguir a primeira tentativa em estimar o modelo dinâmico BIZU via MCMC. O procedimento não se mostrou o mais adequado devido a dificuldade em obter a distribuição preditiva $\left(Y_{t} \mid D_{t-1}\right)$ e a limitação de que esta não pode ser obtida de forma sequencial.

Foi gerada uma série temporal com dados $B I Z U$ com dinâmica tanto no parâmetro de média como no parâmetro de precisão. Para o parâmetro de média foi especificado um modelo com nível (level), tendência (growth) e sazonalidade (seasonality) (vide da-Silva et al., 2011) e para o parâmetro de precisão foi especificado o modelo com nível e tendência.

O modelo dinâmico $B I Z U$ foi ajustado na série temporal, onde os valores iniciais para os parâmetros latentes foram obtidos via MCMC, os espaços de estados foram obtidos via FBBS e os parâmetros estáticos estimados via amostrador de Gibbs a partir das distribuições condicionais completas descritas acima. O modelo foi ajustado prevendo os efeitos de nível, tendência e sazonalidade para a média e o efeito de nível e tendência para a precisão.

Uma dificuldade na estimativa via MCMC é na escolha de distribuição geradora de candidatos para o preditor linear $\eta_{t}$, uma vez que geramos sem levar em consideração a observação $y_{t}$. Para contornar esta situação, McCormick et al. (2012) propõe uma linearização para obter $p\left(\eta_{t} \mid y_{t}, D_{t-1}, \theta_{t-1}\right)$. É utilizada a aproximação $p\left(\eta_{t} \mid y_{t}, D_{t-1}, \theta_{t-1}\right) \approx N\left(\hat{\eta}_{t}, \hat{\Sigma}_{t}\right)$, onde

$$
\begin{aligned}
\hat{\eta}_{t} & =\hat{\eta}_{t-1}-\partial^{2} l\left(\hat{\eta}_{t-1}\right) \partial l\left(\hat{\eta}_{t-1}\right) ; \\
\hat{\Sigma}_{t} & =-\left\{\partial^{2} l\left(\hat{\eta}_{t-1}\right)\right\}^{-1}
\end{aligned}
$$

$\operatorname{com} l=\log \left[p\left(y_{t} \mid \eta_{t}\right) \times p\left(\eta_{t} \mid, D_{t-1}, \theta_{t-1}\right)\right], \partial l$ e $\partial^{2} l$ sua primeira e segunda derivada respectivamente. Souza (2014) utiliza esta linearização como geradora de candidatos e apresenta melhoria no procedimento de estimação via MCMC.

O ajuste dos preditores lineares podem ser observados na Figura 4.4. Além disso o procedimento se mostrou bastante sensível nas prioris do modelo, de modo que em algumas variações das prioris não foi possível e obter convergência. Devido a perda da natureza sequencial dos modelos dinâmicos e a dificuldade em obter a distribuição preditiva do modelo, optamos por não prosseguir com a estimação via MCMC. A estimação via filtro de partículas será a metodologia utilizada para os modelos dinâmicos abordados neste trabalho.

O capítulo 5 apresenta detalhes da estimação de modelos dinâmicos via filtro de partículas que possui vantagens em relação ao custo computacional e por preservar a natureza sequencial de estimação e previsão dos modelos dinâmicos. A estimativa via filtro de partículas desta mesma estrutura do modelo BIZU dinâmico é apresentada mais a frente na Seção (6.1.2). 

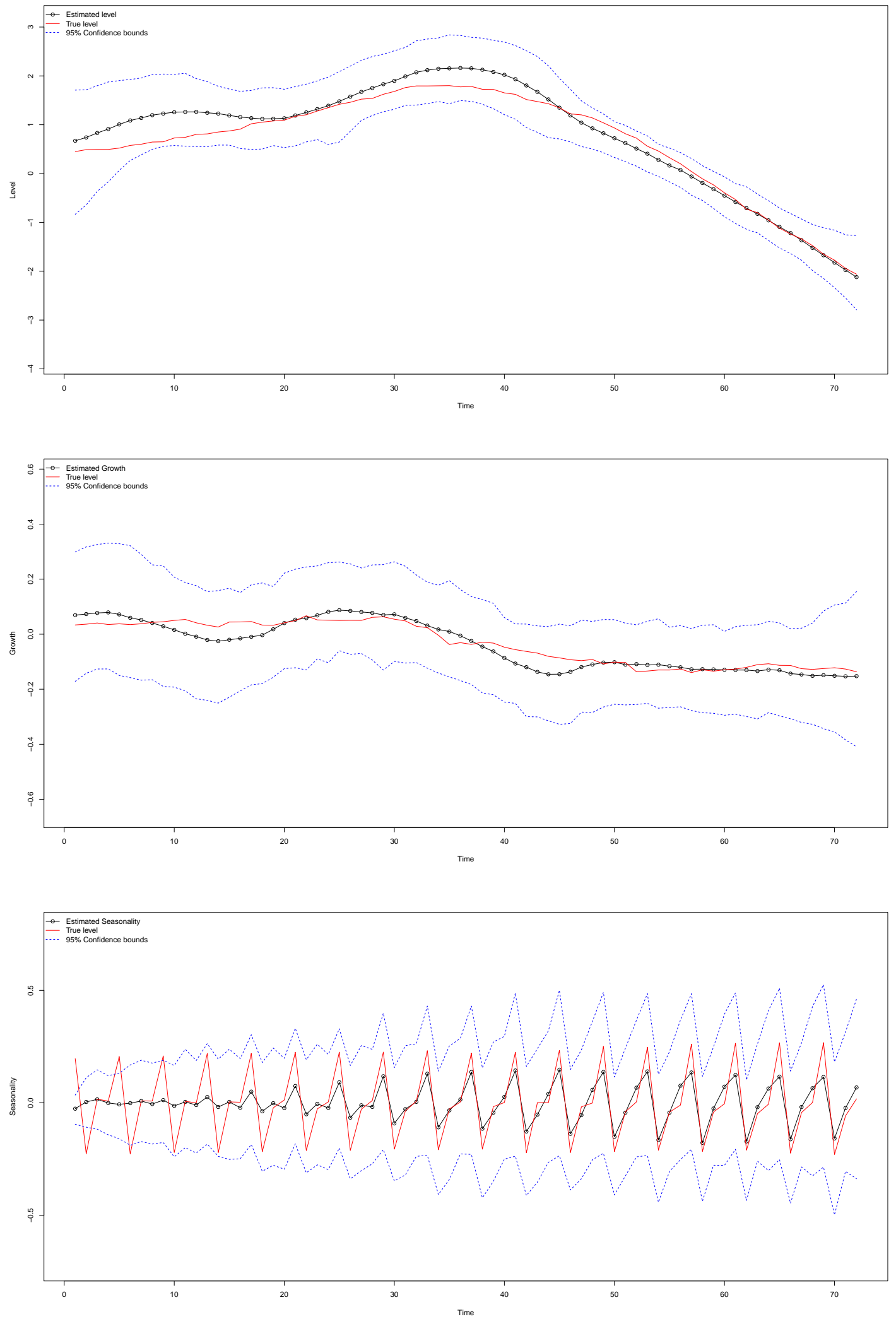

Figura 4.2: Gráfico de ajuste dos efeitos de nível, tendência e sazonalidade para a média com bandas de confiança de $95 \%$. 

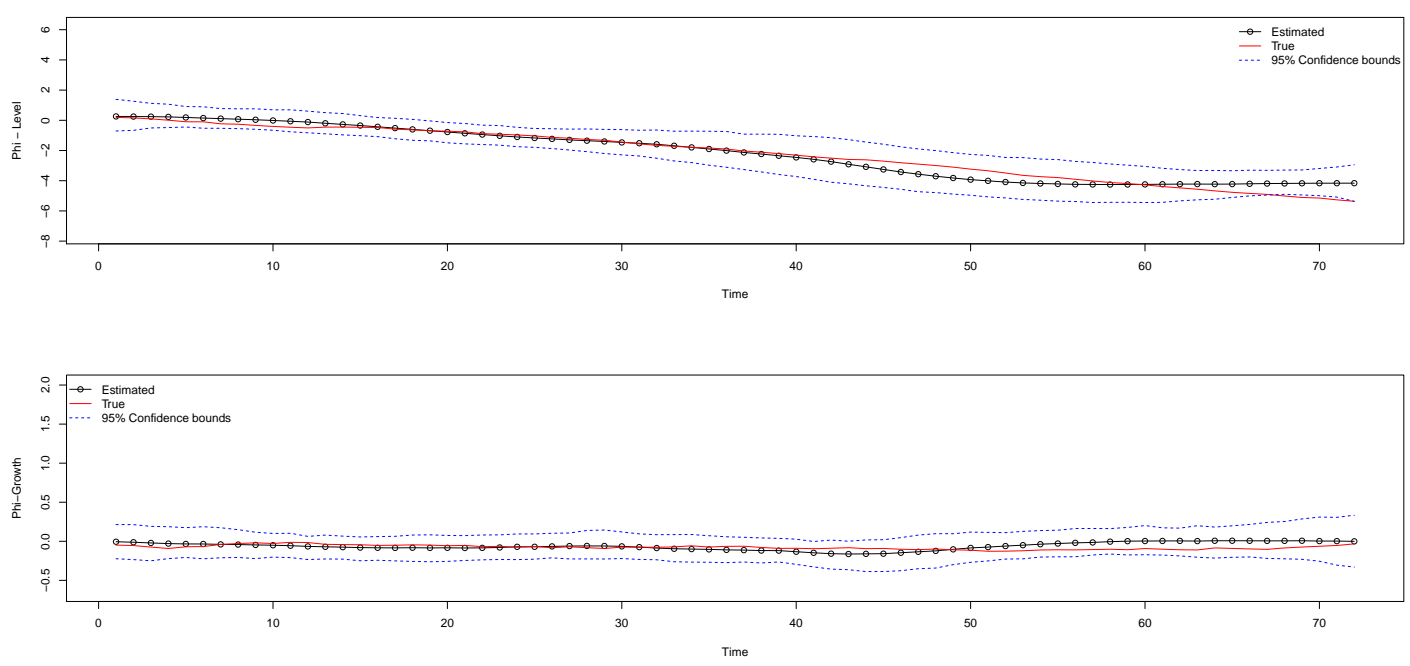

Figura 4.3: Gráfico de ajuste dos efeitos de nível e tendência para a precisão com bandas de confiança de $95 \%$.
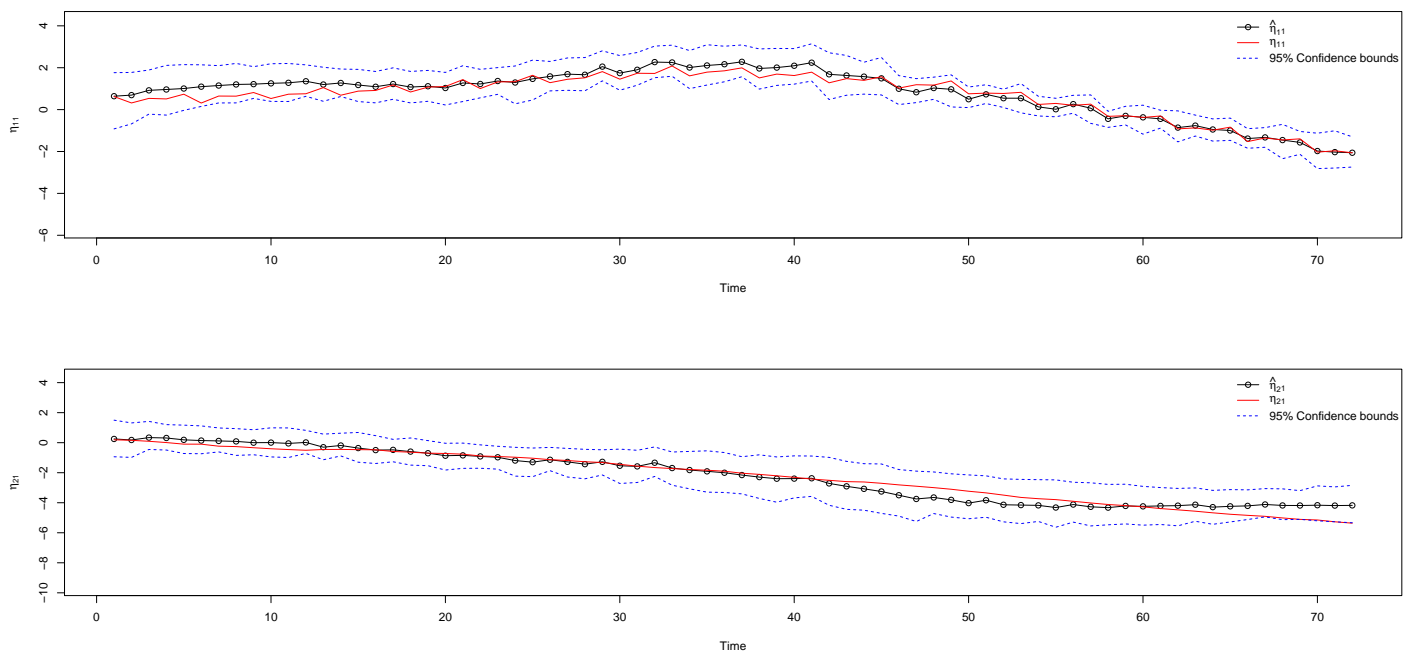

Figura 4.4: Gráfico de ajuste dos preditores lineares do modelo com bandas de confiança de $95 \%$. 

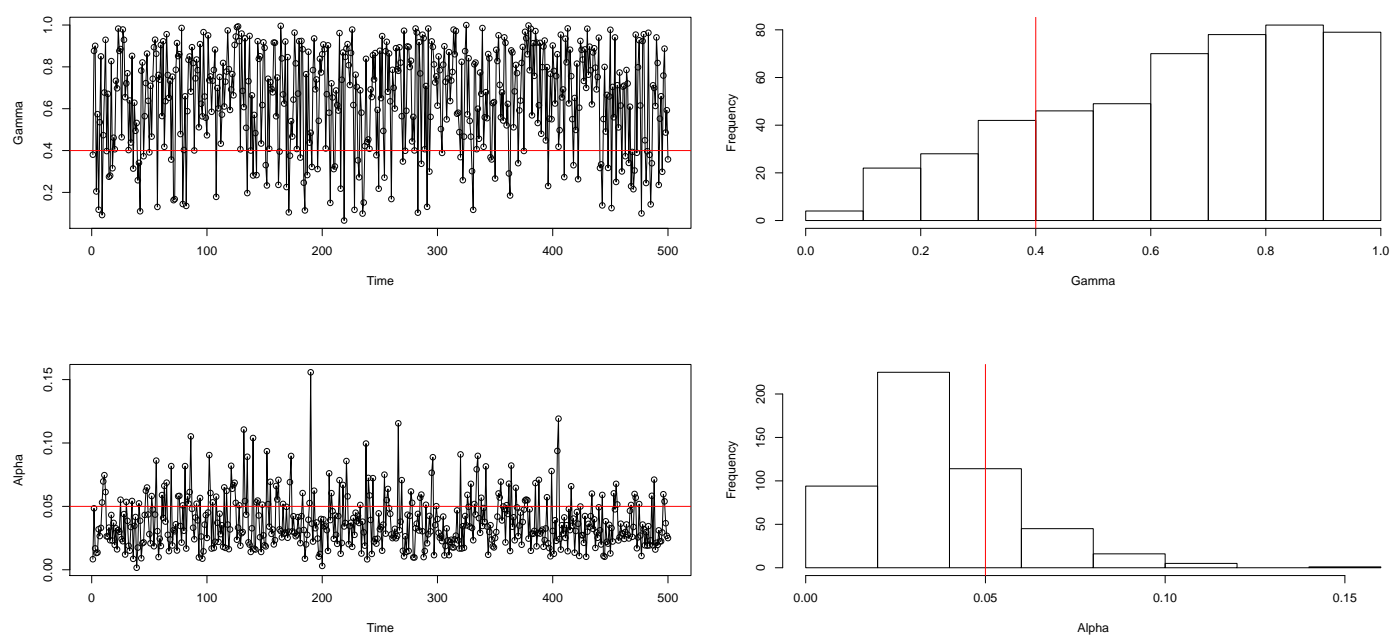

Figura 4.5: Gráfico das distribuições a posteriori dos parâmetros estáticos $\alpha$ e $\gamma$. 


\subsection{Modelo dinâmico Tobit-normal}

O modelo dinâmico Tobit apresentado por Wei (1999) é estruturado da seguinte forma.

Considere $y_{1}, \ldots, y_{T}$ uma série temporal onde

$$
Y_{t}=\left\{\begin{array}{l}
Y_{t}^{*} ; \quad \text { se } Y_{t}^{*}>0 \\
0 ; \quad \text { se } Y_{t}^{*} \leq 0
\end{array}\right.
$$

e temos a seguinte estrutura do modelo dinâmico Tobit-normal:

- Equação das observações:

$$
y_{t}^{*}=\boldsymbol{F}_{t}^{\prime} \boldsymbol{\theta}_{t}+v_{t} ; \quad v_{t} \sim N(0, \boldsymbol{V})
$$

- Equação do sistema:

$$
\boldsymbol{\theta}_{t}=\boldsymbol{G}_{t} \boldsymbol{\theta}_{t-1}+\boldsymbol{w}_{t} ; \quad v_{t} \sim N(0, \boldsymbol{W})
$$

- Equação do sistema:

$$
\left(\boldsymbol{\theta}_{0} \mid D_{0}\right) \sim N\left(\boldsymbol{m}_{0}, \boldsymbol{C}_{0}\right)
$$

Em que

$$
\begin{aligned}
p\left(y_{t} \mid y_{t}^{*}, D_{t-1}, \boldsymbol{\theta}_{t}, \boldsymbol{V}\right) & =\left[P\left(y_{t}^{*} \leq 0 \mid D_{t-1}, \boldsymbol{\theta}_{t}, \boldsymbol{V}\right)\right]^{I_{0 t}}\left[f\left(y_{t}^{*}\right)\right]^{1-I_{0 t}} \\
& =\left[\Phi\left(-\frac{\boldsymbol{F}_{t}^{\prime} \boldsymbol{\theta}_{t}}{\sqrt{\boldsymbol{V}}}\right)\right]^{I_{0 t}}\left[\frac{1}{\sqrt{\boldsymbol{V}}} \phi\left(\frac{y_{t}^{*}-\boldsymbol{F}_{t}^{\prime} \boldsymbol{\theta}_{t}}{\sqrt{\boldsymbol{V}}}\right)\right]^{1-I_{0 t}},
\end{aligned}
$$

onde $I_{0 t}=\mathbb{1}\left(y_{t}^{*} \leq 0\right)$, com $I_{0 t}$ observado. Temos portanto, $\left(y_{t}, I_{0 t}\right)$ observado e $y_{t}^{*}$ como variável latente.

Em resumo, estamos trabalhando com as seguintes notações

- $\boldsymbol{\theta}_{t}$ é o vetor s-dimensional de estados latentes;

- $\boldsymbol{F}_{t}$ é um vetor de dimensão $s$;

- $v_{t}$ corresponde ao erro da equação estrutural;

- $V$ é a variância de $v_{t}$;

- $\boldsymbol{G}_{t}$ é matriz $s \times s$ que especifica os efeitos nos estados latentes;

- $\boldsymbol{w}_{t}$ é o vetor de erros da equação do sistema;

- $\boldsymbol{W}$ é matriz $s \times s$ bloco-diagonal de covariância de $\boldsymbol{w}_{t}$;

- $\boldsymbol{\theta}=\left(\boldsymbol{\theta}_{0}, \ldots, \boldsymbol{\theta}_{T}\right)$;

- $\boldsymbol{y}^{*}=\left(y_{0}^{*}, \ldots, y_{T}^{*}\right)$;

- $\boldsymbol{y}=\left(y_{1}, \ldots, y_{T}\right)$;

- $\psi_{0}=V^{-1}$;

- $\boldsymbol{\psi}_{1}=\boldsymbol{W}^{-1}$. 


\section{Análise Bayesiana}

Vamos considerar as seguintes distribuições a priori:

$$
\begin{aligned}
\psi_{0} & \sim \operatorname{Gama}\left(\nu_{0}, S_{0}\right) \\
\boldsymbol{\psi}_{1} & \sim \operatorname{Wishart}\left(\nu_{1}, S_{1}\right) ; \\
y_{t}^{*} & \sim N\left(\boldsymbol{F}_{t}^{\prime} \boldsymbol{\theta}_{t}, \psi_{0}^{-1}\right) ; \\
\boldsymbol{\theta}_{t} & \sim N\left(\boldsymbol{G}_{t} \boldsymbol{\theta}_{t-1}, \boldsymbol{\psi}_{1}^{-1}\right) .
\end{aligned}
$$

Desta forma, temos a distribuição a posteriori conjunta:

$$
\begin{aligned}
p\left(\boldsymbol{y}^{*}, \boldsymbol{\theta}, \psi_{0}, \boldsymbol{\psi}_{1} \mid \boldsymbol{y}\right) \propto & \left\{\prod_{t=1}^{T}\left[\Phi\left(-\frac{\boldsymbol{F}_{t}^{\prime} \boldsymbol{\theta}_{t}}{\sqrt{\psi_{0}^{-1}}}\right)\right]^{I_{0 t}}\left[\frac{1}{\sqrt{\psi_{0}}-1} \phi\left(\frac{y_{t}^{*}-\boldsymbol{F}_{t}^{\prime} \boldsymbol{\theta}_{t}}{\sqrt{\psi_{0}^{-1}}}\right)\right]^{1-I_{0 t}}\right. \\
& \left.\times N\left(y_{t}^{*} ; \boldsymbol{F}_{t}^{\prime} \boldsymbol{\theta}_{t}, \psi_{0}^{-1}\right) \times N\left(\boldsymbol{\theta}_{t} ; \boldsymbol{G}_{t} \boldsymbol{\theta}_{t-1}, \boldsymbol{\psi}_{1}^{-1}\right)\right\} \\
& \times W\left(\psi_{0} ; \nu_{0}, S_{0}\right) \times W\left(\boldsymbol{\psi}_{1} ; \nu_{1}, \boldsymbol{S}_{1}\right) \times N\left(\boldsymbol{\theta}_{0} ; \boldsymbol{m}_{0}, \boldsymbol{C}_{0}\right) .
\end{aligned}
$$

As distribuições condicionais completas a posteriori são obtidas da seguinte forma:

$$
\begin{aligned}
p\left(\boldsymbol{\theta} \mid \boldsymbol{y}^{*}, \psi_{0}, \boldsymbol{\psi}_{1}, \boldsymbol{y}\right) & =p\left(\boldsymbol{\theta}_{T} \mid \boldsymbol{y}^{*}, \psi_{0}, \boldsymbol{\psi}_{1}, \boldsymbol{y}\right) \prod_{t=1}^{T-1} p\left(\boldsymbol{\theta}_{t} \mid \boldsymbol{\theta}_{t+1}, \boldsymbol{y}^{*}, \psi_{0}, \boldsymbol{\psi}_{1}, \boldsymbol{y}\right) \\
& \propto p\left(\boldsymbol{\theta}_{T} \mid \boldsymbol{y}^{*}, \psi_{0}, \boldsymbol{\psi}_{1}, \boldsymbol{y}\right) \prod_{t=1}^{T-1} p\left(\boldsymbol{\theta}_{t+1} \mid \boldsymbol{\theta}_{t}, \boldsymbol{y}^{*}, \psi_{0}, \boldsymbol{\psi}_{1}, \boldsymbol{y}\right) p\left(\boldsymbol{\theta}_{t} \mid \boldsymbol{y}^{*}, \psi_{0}, \boldsymbol{\psi}_{1}, \boldsymbol{y}\right) .
\end{aligned}
$$

Assim todos os vetores de estado podem ser obtidos de $p\left(\boldsymbol{\theta}_{t} \mid \boldsymbol{y}^{*}, \psi_{0}, \boldsymbol{\psi}_{1}, \boldsymbol{y}\right)$ via algoritmo FFBS (foward-filtering backward-sampling) (Carter e Kohn, 1994; Früwirth-Schmatter, 1994) condicionalmente aos valores de $\left(\boldsymbol{y}^{*}, \boldsymbol{y}^{)}\right.$. Este algoritmo permite construir amostras de $\boldsymbol{\theta}_{T}, \boldsymbol{\theta}_{T-1}, \ldots, \boldsymbol{\theta}_{0}$ por

\section{Filtragem:}

Utilizando o filtro de Kalman (De Jong, 1991) obtemos os momentos $\boldsymbol{m}_{t}$ e $\boldsymbol{C}_{t}$ da posteriori conjunta $p\left(\boldsymbol{\theta}_{t} \mid \boldsymbol{y}^{*}, \psi_{0}, \boldsymbol{\psi}_{1}, \boldsymbol{y}\right), t=1, \ldots, T$ aplicando MLD, mais detalhes em West e Harrison (1997).

$$
\begin{aligned}
& \boldsymbol{m}_{t}=\boldsymbol{a}_{t}+\boldsymbol{A}_{t} e_{t} \text { e } \boldsymbol{C}_{t}=\boldsymbol{R}_{t}-\boldsymbol{A}_{t} \boldsymbol{Q}_{t} \boldsymbol{A}_{t}^{\prime} \\
& \boldsymbol{A}_{t}=\boldsymbol{R}_{t}^{\prime} \boldsymbol{F}_{t} \boldsymbol{Q}_{t}^{-1} \text { e } e_{t}=y_{t}^{*}-f_{t} \\
& \boldsymbol{a}_{t}=\boldsymbol{G}_{t} \boldsymbol{m}_{t-1} \text { e } \boldsymbol{R}_{t}=\boldsymbol{G}_{t} \boldsymbol{C}_{t-1} \boldsymbol{G}_{t}^{\prime}+\psi_{1}^{-1} \\
& f_{t}=\boldsymbol{F}_{t}^{\prime} \boldsymbol{a}_{t} \text { e } \boldsymbol{Q}_{t}=\boldsymbol{F}_{t}^{\prime} \boldsymbol{R}_{t} \boldsymbol{F}_{t}+\psi_{0}^{-1} .
\end{aligned}
$$

\section{Suavização:}

No momento $t=T, \boldsymbol{\theta}_{T}$ é obtido de $\left(\boldsymbol{\theta}_{T} \mid \boldsymbol{y}^{*}, \psi_{0}, \boldsymbol{\psi}_{1}, \boldsymbol{y}\right) \sim N\left(\boldsymbol{m}_{T}, \boldsymbol{C}_{T}\right)$. Para os tempos $\{T-$ $1, \ldots, 0\}$, obtemos $\boldsymbol{\theta}_{t}$ de $\left(\boldsymbol{\theta}_{t} \mid \boldsymbol{\theta}_{t+1} \boldsymbol{y}^{*}, \psi_{0}, \boldsymbol{\psi}_{1}, \boldsymbol{y}\right)$ condicionalmente ao valor de $\boldsymbol{\theta}_{t+1}$. Isto é feito amostrando $\boldsymbol{\theta}_{t}$ de $\left(\boldsymbol{\theta}_{t} \mid \boldsymbol{\theta}_{t+1} \boldsymbol{y}^{*}, \psi_{0}, \boldsymbol{\psi}_{1}, \boldsymbol{y}\right) \sim N\left(\boldsymbol{u}_{t}, \boldsymbol{B}_{t}\right)$.

$$
\begin{aligned}
& \boldsymbol{u}_{t}=\boldsymbol{m}_{t}+\boldsymbol{B}_{t}\left(\boldsymbol{\theta}_{t+1}-\boldsymbol{a}_{t+1}\right) ; \\
& \boldsymbol{U}_{t}=\boldsymbol{C}_{t}-\boldsymbol{B}_{t} \boldsymbol{R}_{t+1} \boldsymbol{B}_{t}^{\prime} ; \\
& \boldsymbol{B}_{t}=\boldsymbol{C}_{t} \boldsymbol{G}_{t} \boldsymbol{R}_{t+1}^{\prime} .
\end{aligned}
$$

Temos ainda

$$
\left(y_{t}^{*} \mid \boldsymbol{\theta}_{t}, \psi_{0}, \boldsymbol{\psi}_{1}, y_{t}\right) \sim Y_{t} \mathbb{1}\left(y_{t}>0\right)+\left[\frac{\frac{1}{\sqrt{\psi_{0}^{-1}}} \phi\left(\frac{y_{t}^{*}-\boldsymbol{F}_{t}^{\prime} \boldsymbol{\theta}_{t}}{\sqrt{\psi_{0}^{-1}}}\right)}{\Phi\left(-\frac{\boldsymbol{F}_{t}^{\prime} \boldsymbol{\theta}_{t}}{\sqrt{\psi_{0}^{-1}}}\right)} \mathbb{1}\left(y_{t}^{*} \leq 0\right)\right] \mathbb{1}\left(y_{t}=0\right)
$$


por argumentos de dados aumentados (Lopes, 2012). Temos ainda $\left(\psi_{0} \mid \boldsymbol{\theta}, \boldsymbol{y}^{*}, \boldsymbol{\psi}_{1}, \boldsymbol{y}\right)$ e $\left(\boldsymbol{\psi}_{1} \mid \boldsymbol{\theta}, \boldsymbol{y}^{*}, \psi_{0}, \boldsymbol{y}\right)$ obtidos da mesma forma apresentada em da-Silva e Migon (2012).

$$
\begin{aligned}
p\left(\psi_{0} \mid \boldsymbol{\theta}, \boldsymbol{y}^{*}, \boldsymbol{\psi}_{1}, \boldsymbol{y}\right) \propto & {\left[\prod_{t=1}^{T} N\left(y_{t}^{*} ; \boldsymbol{F}_{t}^{\prime} \boldsymbol{\theta}_{t}, \psi_{0}^{-1}\right)\right] W\left(\psi_{0} ; \nu_{0}, S_{0}\right) } \\
\propto & {\left[\prod_{t=1}^{T}\left|\psi_{0}\right|^{1 / 2} \exp \left\{-\frac{1}{2}\left(y_{t}^{*}-\boldsymbol{F}_{t}^{\prime} \boldsymbol{\theta}_{t}\right)^{\prime} \psi_{0}\left(y_{t}^{*}-\boldsymbol{F}_{t}^{\prime} \boldsymbol{\theta}_{t}\right)\right\}\right] } \\
& \times\left|\psi_{0}\right|^{\nu_{0}-1} \exp \left\{-S_{0} \psi_{0}\right\} \\
\propto & \left|\psi_{0}\right|^{T / 2+\nu_{0}-1} \exp \left\{\left(S S_{y_{t}^{*}} / 2+S_{0}\right) \psi_{0}\right\},
\end{aligned}
$$

$\operatorname{com} S S_{y_{t}^{*}}=\left(y_{t}^{*}-\boldsymbol{F}_{t}^{\prime} \boldsymbol{\theta}_{t}\right)^{2}$ e $S S_{y^{*}}=\sum_{t-1}^{T} S S_{y_{t}^{*}}$. Com isso $\left(\psi_{0} \mid \boldsymbol{\theta}, \boldsymbol{y}^{*}, \boldsymbol{\psi}_{1}, \boldsymbol{y}\right) \sim W\left(T / 2+\nu_{0}, S S_{y^{*}}+S_{0}\right)$. Temos ainda

$$
\begin{aligned}
p\left(\boldsymbol{\psi}_{1} \mid \boldsymbol{\theta}, \boldsymbol{y}^{*}, \psi_{0}, \boldsymbol{y}\right) \propto & {\left[\prod_{t=1}^{T} N\left(\boldsymbol{\theta}_{t} ; \boldsymbol{G}_{t} \boldsymbol{\theta}_{t-1}, \boldsymbol{\psi}_{1}^{-1}\right)\right] W\left(\boldsymbol{\psi}_{1} ; \nu_{1}, \boldsymbol{S}_{1}\right) } \\
\propto & {\left[\prod_{t=1}^{T}\left|\boldsymbol{\psi}_{1}\right|^{1 / 2} \exp \left\{-\frac{1}{2}\left(\boldsymbol{\theta}_{t}-\boldsymbol{G}_{t} \boldsymbol{\theta}_{t-1}\right)^{\prime} \boldsymbol{\psi}_{1}\left(\boldsymbol{\theta}_{t}-\boldsymbol{G}_{t} \boldsymbol{\theta}_{t-1}\right)\right\}\right] } \\
& \times\left|\boldsymbol{\psi}_{1}\right|^{\left.\nu_{1}-s+1\right) / 2} \exp \left\{-\operatorname{tr}\left(\boldsymbol{S}_{1} \boldsymbol{\psi}_{1}\right)\right\} \\
\propto & \left|\boldsymbol{\psi}_{1}\right|^{T / 2+\nu_{1}-(s+1) / 2} \exp \left\{-\operatorname{tr}\left(S S_{\theta} / 2+\boldsymbol{S}_{1}\right) \boldsymbol{\psi}_{1}\right\}
\end{aligned}
$$

$\operatorname{com} S S_{\theta_{t}}=\left(\boldsymbol{\theta}_{t}-\boldsymbol{G}_{t} \boldsymbol{\theta}_{t-1}\right)\left(\boldsymbol{\theta}_{t}-\boldsymbol{G}_{t} \boldsymbol{\theta}_{t-1}\right)^{\prime}$ e $S S_{\theta}=\sum_{t=1}^{T} S S_{\theta_{t}}$. Então $\left(\boldsymbol{\psi}_{1} \mid \boldsymbol{\theta}, \boldsymbol{y}^{*}, \psi_{0}, \boldsymbol{y}\right) \sim W(T / 2+$ $\left.\nu_{1}, S S_{\theta}+\boldsymbol{S}_{1}\right)$.

\section{Distribuição preditiva}

Para análises de previsão, precisamos obter a distribuição preditiva de um passo a frente, $p\left(y_{t} \mid D_{t-1}\right)$, onde

$$
p\left(y_{t} \mid D_{t-1}\right)=\int p\left(y_{t} \mid D_{t-1}, \boldsymbol{\theta}_{t}\right) p\left(\boldsymbol{\theta}_{t} \mid D_{t-1}\right) d \boldsymbol{\theta}_{t} .
$$

Porém

$$
\begin{aligned}
p\left(y_{t} \mid D_{t-1}, \boldsymbol{\theta}_{t}\right)= & \int p\left(y_{t}, y_{t}^{*} \mid D_{t-1}, \boldsymbol{\theta}_{t}\right) d y_{t}^{*} \\
= & \int p\left(y_{t} \mid D_{t-1}, y_{t}^{*}, \boldsymbol{\theta}_{t}\right) p\left(y_{t}^{*} \mid D_{t-1}, \boldsymbol{\theta}_{t}\right) d y_{t}^{*} \\
= & p\left(y_{t} \mid D_{t-1}, \boldsymbol{\theta}_{t}, y_{t}^{*}>0\right)\left(1-F\left(0 \mid D_{t-1}, \boldsymbol{\theta}_{t}\right)\right) \\
& +p\left(y_{t} \mid D_{t-1}, \boldsymbol{\theta}_{t}, y_{t}^{*} \leq 0\right) F\left(0 \mid D_{t-1}, \boldsymbol{\theta}_{t}\right) \quad \text { onde, } \\
F\left(0 \mid D_{t-1}, \boldsymbol{\theta}_{t}\right)= & F\left(y_{t}^{*} \leq 0 \mid D_{t-1}, \boldsymbol{\theta}_{t}\right) \\
= & \Phi\left(-\frac{\boldsymbol{F}_{t}^{\prime} \boldsymbol{\theta}_{t}}{\sqrt{\boldsymbol{V}}}\right) .
\end{aligned}
$$


Logo,

$$
\begin{aligned}
p\left(y_{t} \mid D_{t-1}, \boldsymbol{\theta}_{t}\right)= & p\left(y_{t} \mid D_{t-1}, \boldsymbol{\theta}_{t}, y_{t}^{*}>0\right)\left(1-\Phi\left(-\frac{\boldsymbol{F}_{t}^{\prime} \boldsymbol{\theta}_{t}}{\sqrt{\boldsymbol{V}}}\right)\right) \\
& +p\left(y_{t} \mid D_{t-1}, \boldsymbol{\theta}_{t}, y_{t}^{*} \leq 0\right) \Phi\left(-\frac{\boldsymbol{F}_{t}^{\prime} \boldsymbol{\theta}_{t}}{\sqrt{\boldsymbol{V}}}\right) \\
= & \begin{cases}\frac{1}{\sqrt{V}} \phi\left(-\frac{y_{t}-\boldsymbol{F}_{t}^{\prime} \boldsymbol{\theta}_{t}}{\sqrt{\boldsymbol{V}}}\right)\left[1-\Phi\left(-\frac{\boldsymbol{F}_{t}^{\prime} \boldsymbol{\theta}_{t}}{\sqrt{\boldsymbol{V}}}\right)\right] ; & y_{t}>0, \\
{\left[\Phi\left(-\frac{\boldsymbol{F}_{t}^{\prime} \boldsymbol{\theta}_{t}}{\sqrt{\boldsymbol{V}}}\right)\right]^{2} ;} & y_{t}=0 .\end{cases}
\end{aligned}
$$

Há necessidade de integração não apenas no espaço dos estados $\boldsymbol{\theta}=\left(\boldsymbol{\theta}_{1}, \ldots, \boldsymbol{\theta}_{T}\right)$, mas, também, no espaço das matrizes $\boldsymbol{V}$ e $\boldsymbol{W}$ torna o problema de determinação da distribuição preditiva bastante complexo, como é possivel observar na expressão a seguir:

$$
p\left(y_{t} \mid D_{t-1}, \boldsymbol{V}, \boldsymbol{W}\right)=\iiint p\left(y_{t} \mid D_{t-1}, \boldsymbol{\theta}_{t}, \boldsymbol{\psi}_{0}\right) p\left(\boldsymbol{\theta}_{t} \mid D_{t-1}, \boldsymbol{\phi}_{1}\right) p\left(\boldsymbol{\phi}_{0}\right) p\left(\boldsymbol{\phi}_{1}\right) d \boldsymbol{\theta}_{t} .
$$

A forma geral para previsão a $k$-passos a frente é obtida pelas Equações (4.3) e (4.4).

Wei (1999) propõe uma estimação via amostrador de Gibbs reordenando a série temporal, agrupando os dados completos e os dados censurados. No entanto, para o contexto de dados de limitados, ou mais especificamente dados de taxa ou proporção, sugerimos algumas modificações na estrutura do modelo. Na seção 4.3.1 é detalhado o modelo Tobit com esta nova formulação.

\subsubsection{Modelo dinâmico Tobit normal duplamente censurado}

Considere $y_{1}, \ldots, y_{T}$ uma série temporal onde

$$
Y_{t}=\left\{\begin{array}{l}
0 ; \quad \text { se } Y_{t}^{*} \leq 0 \\
Y_{t}^{*} ; \quad \text { se } 0<Y_{t}^{*}<1 \\
1 ; \quad \text { se } Y_{t}^{*} \geq 1
\end{array}\right.
$$

com $E\left[Y_{t}^{*}\right]=\mu_{t}$ e $V\left[Y_{t}^{*}\right]=\sigma_{t}^{2}$. Desta forma, temos a seguinte estrutura do modelo dinâmico Tobit-normal:

\section{- Transformações nos parâmetros com dinâmica:}

$$
\eta_{1 t}=h_{1}\left(\mu_{t}\right) ; \quad \eta_{2 t}=h_{2}\left(\sigma_{t}\right)
$$

Podemos considerar as opções das funções de ligação logito e log:

$$
h_{1}\left(\mu_{t}\right)=\log \left(\frac{\mu_{t}}{1-\mu_{t}}\right) \quad h_{2}\left(\sigma_{t}\right)=\log \left(\sigma_{t}\right)
$$

- Equação das estrutural:

$$
\eta_{t}=\boldsymbol{F}_{t}^{\prime} \boldsymbol{\theta}_{t}
$$

- Equação do sistema:

$$
\boldsymbol{\theta}_{t}=\boldsymbol{G}_{t} \boldsymbol{\theta}_{t-1}+\boldsymbol{w}_{t} ; \quad v_{t} \sim N(0, \boldsymbol{W})
$$

- Priori inicial:

$$
\left(\boldsymbol{\theta}_{0} \mid D_{0}\right) \sim N\left(\boldsymbol{m}_{0}, \boldsymbol{C}_{0}\right) .
$$


Em que

$$
\begin{aligned}
p\left(y_{t} \mid y_{t}^{*}, D_{t-1}, \boldsymbol{\theta}_{t}\right) & =\left[P\left(y_{t}^{*} \leq 0 \mid D_{t-1}, \boldsymbol{\theta}_{t}\right)\right]^{I_{0 t}}\left[P\left(y_{t}^{*} \geq 1 \mid D_{t-1}, \boldsymbol{\theta}_{t}\right)\right]^{I_{1 t}}\left[f\left(y_{t}^{*}\right)\right]^{1-I_{0 t}-I_{1 t}} \\
& =\left[\Phi\left(-\frac{\mu_{t}}{\sigma_{t}}\right)\right]^{I_{0 t}}\left[1-\Phi\left(\frac{\mu_{t}}{\sigma_{t}}\right)\right]^{I_{1 t}}\left[\frac{1}{\sigma_{t}} \phi\left(\frac{y_{t}^{*}-\mu_{t}}{\sigma_{t}}\right)\right]^{1-I_{0 t}-I_{1 t}}
\end{aligned}
$$

onde $I_{0 t}=\mathbb{1}\left(y_{t}^{*} \leq 0\right), I_{1 t}=\mathbb{1}\left(y_{t}^{*} \geq 1\right)$, com $I_{0 t}$ e $I_{1 t}$ observados. Temos portanto, $\left(y_{t}, I_{0 t}, I_{1 t}\right)$ observado e $y_{t}^{*}$ como variável latente.

Em resumo, estamos trabalhando com as seguintes notações

- $\boldsymbol{\theta}_{t}$ é o vetor s-dimensional de estados latentes;

- $\boldsymbol{\eta}_{t}=\left(\eta_{1 t}, \eta_{2 t}\right)^{\prime}$ é vetor $2 \times 1$ de parâmetros estruturais;

- $\boldsymbol{F}_{t}=\left(\boldsymbol{F}_{1 t}^{\prime}, \boldsymbol{F}_{2 t}^{\prime}\right)^{\prime}$ é matriz $2 \times s$;

- $\boldsymbol{G}_{t}$ é matriz $s \times s$ que especifica os efeitos nos estados latentes;

- $\boldsymbol{w}_{t}$ é o vetor de erros da equação do sistema;

- $\boldsymbol{W}$ é matriz $s \times s$ bloco-diagonal de covariância de $\boldsymbol{w}_{t}$;

- $\boldsymbol{\theta}=\left(\boldsymbol{\theta}_{0}, \ldots, \boldsymbol{\theta}_{T}\right)$;

- $\boldsymbol{y}^{*}=\left(y_{0}^{*}, \ldots, y_{T}^{*}\right)$;

- $\boldsymbol{y}=\left(y_{1}, \ldots, y_{T}\right)$;

- $\boldsymbol{\psi}_{1}=\boldsymbol{W}^{-1}$.

Temos ainda as condicionais completas, onde $\left(\boldsymbol{\theta} \mid \boldsymbol{\eta}, \boldsymbol{y}^{*}, \boldsymbol{\psi}_{1}, \boldsymbol{y}\right)$ é obtido via Filtro de Kalman. Temos ainda

$$
\begin{aligned}
\left(y_{t}^{*} \mid \boldsymbol{\theta}_{t}, \boldsymbol{\psi}_{1}, y_{t}\right) \sim & Y_{t} \mathbb{1}\left(0<y_{t}<1\right)+\left[\frac{\frac{1}{\sigma_{t}} \phi\left(\frac{y_{t}^{*}-\mu_{t}}{\sigma_{t}}\right)}{\Phi\left(-\frac{\mu_{t}}{\sigma_{t}}\right)} \mathbb{1}\left(y_{t}^{*} \leq 0\right)\right] \mathbb{1}\left(y_{t}=0\right) \\
+ & {\left[\frac{\frac{1}{\sigma_{t}} \phi\left(\frac{y_{t}^{*}-\boldsymbol{F}_{t}^{\prime} \boldsymbol{\theta}_{t}}{\sqrt{\psi_{0}^{-1}}}\right)}{1-\Phi\left(\frac{1-\mu_{t}}{\sigma_{t}}\right)} \mathbb{1}\left(y_{t}^{*} \geq 1\right)\right] \mathbb{1}\left(y_{t}=1\right) }
\end{aligned}
$$

por argumentos de dados aumentados.

De forma análoga ao Tobit simples, a distribuição preditiva é dada por

$$
\begin{aligned}
p\left(y_{t} \mid D_{t-1}, \boldsymbol{\theta}_{t}\right)= & p\left(y_{t} \mid D_{t-1}, \boldsymbol{\theta}_{t}, 0<y_{t}^{*}<1\right)\left(\Phi\left(\frac{1-\mu_{t}}{\sigma_{t}}\right)-\Phi\left(-\frac{\mu_{t}}{\sigma_{t}}\right)\right) \\
& +p\left(y_{t} \mid D_{t-1}, \boldsymbol{\theta}_{t}, y_{t}^{*} \leq 0\right) \Phi\left(-\frac{\mu_{t}}{\sigma_{t}}\right) \\
& +p\left(y_{t} \mid D_{t-1}, \boldsymbol{\theta}_{t}, y_{t}^{*} \geq 1\right)\left(1-\Phi\left(\frac{1-\mu_{t}}{\sigma_{t}}\right)\right) \\
= & \begin{cases}\frac{1}{\sigma_{t}} \phi\left(-\frac{y_{t}-\mu_{t}}{\sigma_{t}}\right)\left[\Phi\left(\frac{1-\mu_{t}}{\sigma_{t}}\right)-\Phi\left(-\frac{\mu_{t}}{\sigma_{t}}\right)\right] ; & 0<y_{t}<1, \\
{\left[\Phi\left(-\frac{\mu_{t}}{\sigma_{t}}\right)\right]^{2} ;} & y_{t}=0, \\
{\left[1-\Phi\left(\frac{1-\mu_{t}}{\sigma_{t}}\right)\right]^{2} ;} & y_{t}=1 .\end{cases}
\end{aligned}
$$


Nota-se que para obter a distribuição preditiva $p\left(y_{t} \mid D_{t-1}\right)$ envolve integrações que não são possíveis de calcular analiticamente. Para previsões a $k$-passos a frente envolve o processo recursivo descrito na Seção (4.2.1). Métodos de integração por aproximação como quadratura gaussiana como utilizado por da-Silva et al. (2011) são difíceis de se implementar devida a grande quantidade de integrações e a dimensão dos parâmetros. Procedimentos via simulação são os mais adequados para solucionar este tipo de integração. Como no caso do modelo Tobit usual, o procedimento de MCMC pode ser utilizado de forma semelhante a utilizada por Wei (1999). Em contrapartida, a utilização de algoritmos com metodologia Metropolis-Hastings esbarram na dificuldade em obter convergência em integrais com grande número de dimensões, além de não possuir uma estrutura sequencial de predição. Isto é, a cada nova observação na série é necessário estimar toda a série novamente para obter a distribuição preditiva atualizada.

A alternativa mais viável para contornar estas dificuldades é o uso da metodologia Monte Carlo Sequencial (SMC). Gordon et al. (1993) introduziu a técnica hoje conhecida como filtro de partículas como um procedimento Bayesiano recursivo que permite uma redução do custo computacional e um processo de previsão sequencial, sem necessidade de estimar todos os estados da série a cada nova observação. O capítulo 5 apresenta detalhes da estimação via filtro de partículas. 


\section{Capítulo 5}

\section{Filtro de Partículas}

Com o surgimento de diversos modelos de espaços de estados sem solução analítica, metodologias on-line como filtro de Kalman e suas variações passaram a ser insuficientes para o desenvolvimento de suas estimações. No contexto Bayesiano, metodologias da classe MCMC possuem alto custo computacional para estimar séries temporais e o procedimento se torna ainda mais exaustivo pela necessidade de uma nova estimativa de toda a série a cada vez que é inserida uma nova informação no modelo, como por exemplo uma nova observação. Como alternativa para contornar este custo o Monte Carlo sequencial (SMC), e mais especificamente via filtro de partículas, tornou-se uma alternativa interessante para modelos dinâmicos com abordagem Bayesiana.

\subsection{Caso geral}

Considere a estrutura geral de um modelo de espaço de estados:

- Equação de observações

$$
y_{t} \sim p\left(y_{t} \mid x_{t}, \theta_{1}\right)
$$

- Equação do sistema

$$
x_{t} \sim p\left(x_{t} \mid x_{t-1}, \theta_{2}\right)
$$

onde $y_{t}$ representa a observação no tempo $t$ e $x_{t}$ representa o estado latente (não observável) no tempo $t, \operatorname{com} t=1, \ldots, n$.

Nos procedimentos de estimação on-line, a estimação de $x_{t}$ é baseada na observação dos dados $y_{1: t}$. Em um primeiro momento, considere os parâmetros fixos $\boldsymbol{\theta}$ como conhecidos. Assim como nos casos gerais de análise Bayesiana, estamos interessados em obter a distribuição a posteriori dos estados latentes até o tempo presente $t$, isto é,

$$
p\left(x_{1: t} \mid y_{1: t}\right) .
$$

No processo de estimação por filtro de partículas esta distribuição é obtida por meio de um conjunto finito de amostras (partículas) e da estimação Monte Carlo sequencial. Com a chegada de uma nova observação, é possível atualizar as partículas para obter a nova posteriori

$$
p\left(x_{1: t+1} \mid y_{1: t+1}\right) .
$$

Com base na técnica de amostragem por importância sequencial (SIS) o procedimento básico de filtro de partículas segue da seguinte forma:

Estamos interessados em obter uma aproximação numérica de $p\left(x_{1: n} \mid y_{1: n}\right)$ sequencialmente.

Considere $p\left(x_{1: n} \mid y_{1: n}\right)$ a distribuição alvo, dada por

$$
p\left(x_{1: n} \mid y_{1: n}\right)=\frac{p\left(x_{1: n}, y_{1: n}\right)}{p\left(y_{1: n}\right)}
$$


desta forma, temos

$$
\begin{aligned}
p\left(x_{1: n} \mid y_{1: n}\right) & =\frac{p\left(x_{1: n}, y_{1: n}\right)}{\int p\left(x_{1: n}, y_{1: n}\right) d x_{1: n}} \\
& =\frac{p\left(x_{1: n-1} \mid y_{1: n-1}\right) p\left(x_{n} \mid x_{n-1}\right) p\left(y_{n} \mid x_{n}\right)}{\int p\left(x_{1: n-1} \mid y_{1: n-1}\right) p\left(x_{n} \mid x_{n-1}\right) p\left(y_{n} \mid x_{n}\right) d x_{1: n}} .
\end{aligned}
$$

Considere a densidade de importância $q_{n}\left(x_{1: n}\right)$, tem-se:

$$
\begin{aligned}
p\left(x_{1: n} \mid y_{1: n}\right) & =\frac{p\left(x_{1: n}, y_{1: n}\right)}{q_{n}\left(x_{1: n}\right.} \frac{q_{n}\left(x_{1: n}\right.}{p\left(y_{1: n}\right)} \\
& =w_{n}\left(x_{1: n}\right) \frac{q_{n}\left(x_{1: n}\right)}{p\left(y_{1: n}\right)} .
\end{aligned}
$$

Seja $\gamma_{n}\left(x_{1: n}\right)=p\left(x_{1: n}, y_{1: n}\right)$ e considere $w_{n}\left(x_{1: n}\right)$ o peso de importância que pode ser descrito da seguinte forma sequencial:

$$
\begin{aligned}
w_{n}\left(x_{1: n}\right) & =\frac{\gamma_{n}\left(x_{1: n}\right)}{q_{n}\left(x_{1: n}\right)} \\
& =\frac{\gamma_{n-1}\left(x_{1: n-1}\right)}{q_{n-1}\left(x_{1: n-1}\right)} \times \frac{\gamma_{n}\left(x_{1: n}\right)}{\gamma_{n-1}\left(x_{1: n-1}\right) q_{n}\left(x_{n} \mid x_{1: n-1}\right)} \\
& =w_{n-1}\left(x_{1: n}\right) \times \alpha_{n}\left(x_{1: n}\right),
\end{aligned}
$$

com $\alpha_{n}$ denominado o peso de incremento, que pode ser expresso por:

$$
\begin{aligned}
\alpha_{n}\left(x_{1: n}\right) & =\frac{\gamma_{n}\left(x_{1: n}\right)}{\gamma_{n-1}\left(x_{1: n-1}\right) q_{n}\left(x_{n} \mid x_{1: n-1}\right)} \\
& =\frac{p\left(x_{1: n-1} \mid y_{1: n-1}\right) p\left(x_{n} \mid x_{1: n-1}\right) p\left(y_{n} \mid x_{n}\right)}{p\left(x_{1: n-1} \mid y_{1: n-1}\right) q\left(x_{n} \mid y_{n}, x_{n-1}\right)} \\
& =\frac{p\left(x_{n} \mid x_{n-1}\right) p\left(y_{n} \mid x_{n}\right)}{q\left(x_{n} \mid y_{n}, x_{n-1}\right)}=\alpha_{n}\left(x_{n-1}, x_{n}\right)
\end{aligned}
$$

A escolha de $q_{n}\left(x_{1: n}\right)$ deve ser feita com o intuito de minimizar a variância dos pesos de importância $w_{n}($.$) . A densidade de importância ótima é dada por:$

$$
\begin{aligned}
q_{n}^{\text {opt }}\left(x_{n} \mid x_{1: n-1}\right) & =p\left(x_{n} \mid y_{n}, x_{n-1}\right) \\
& =\frac{p\left(y_{n} \mid x_{n}\right) p\left(x_{n} \mid x_{n-1}\right)}{p\left(y_{n} \mid x_{n-1}\right)} .
\end{aligned}
$$

No entanto, na maioria dos casos não é possível amostrar diretamente de $q_{n}^{\text {opt }}\left(x_{n} \mid x_{1: n-1}\right)$. Ainda assim, a equação acima sugere que distribuição de importância deve ser da forma:

$$
q_{n}\left(x_{n} \mid x_{n-1}\right)=q_{n}\left(x_{n} \mid y_{n}, x_{n-1}\right),
$$

uma vez que não há interesse em utilizar uma distribuição que dependa de $\left(y_{1: n-1}, x_{1: n-2}\right)$.

Por fim, obtemos

$$
\hat{p}\left(x_{1: n} \mid y_{1: n}\right)=\frac{\sum_{i=1}^{N} w_{n}\left(x_{n}^{i}\right) \delta\left(x_{1: n}=x_{1: n}^{i}\right)}{\sum_{i=1}^{N} w_{n}\left(x_{n}^{i}\right)},
$$

$\operatorname{com} w_{n}\left(x_{1: n}\right)=w_{n-1}\left(x_{1: n-1}\right) \times \alpha_{n}\left(x_{1: n}\right)$ e $\frac{p\left(x_{n} \mid x_{n-1}\right) p\left(y_{n} \mid x_{n}\right)}{q\left(x_{n} \mid y_{n}, x_{n-1}\right)}$. Ou seja,

$$
\hat{p}\left(x_{1: n} \mid y_{1: n}\right)=\sum_{i=1}^{N} W_{n}\left(x_{1: n}^{i}\right) \delta\left(x_{1: n}=x_{1: n}^{i}\right), \quad \operatorname{com} \quad W_{n}\left(x_{1: n}^{i}\right)=\frac{w_{n}\left(x_{n}^{i}\right)}{\sum_{i=1}^{N} w_{n}\left(x_{n}^{i}\right)}
$$


e $\delta\left(x=x_{0}\right)$ corresponde a função de Dirac no ponto $x_{0}$.

Em resumo, temos o seguinte algoritmo:

- tome $t=1$;

- $\operatorname{amostre} X_{1: t}^{i} \sim q_{n}\left(x_{1: t}\right)$;

- calcule $w_{n}\left(X_{1: t}^{i}\right)=w_{t-1}\left(x_{1: t-1}\right) \times \alpha_{n}\left(x_{1: t}\right) e W_{n}\left(x_{1: t}^{i}\right) \propto w_{n}\left(X_{1: t}^{i}\right)$

- $t=t+1$, até $t=n$.

Por fim, obtemos o conjunto de partículas

$$
S_{t}=\left\{\left(X_{1: t}^{i}, w_{1: n}^{i}\right), i=1, \ldots, N\right\}, \quad t=1, \ldots, n .
$$

Cabe ressaltar que o procedimento de amostragem por importância sequencial (SIS) conduz a estimativas cujas variâncias aumentam com $n$. Com isso, o uso de reamostragem torna-se um procedimento importante para amenizar este problema nos métodos de Monte Carlo sequencial, além de evitar em muitos casos a degeneração dos pesos de importância. Douc e Cappe (2006) apresentam mais detalhes sobre os métodos de reamostragem.

\subsubsection{Filtro no contexto da modelagem Bayesiana}

Considere um modelo de espaço de estados não necessariamente linear. O interesse está na estimação da variável latente $\theta_{t} \in \mathbb{R}^{n}$. Seja $f: \mathbb{R}^{n} \times \mathbb{R}^{m} \Rightarrow \mathbb{R}^{n}$ uma função com propriedades recursivas em $t=1, \ldots, n$, tal que:

$$
\theta_{t}=f\left(\theta_{t-1}, \omega_{t}\right),
$$

com $\omega_{t} \in \mathbb{R}^{m}$ um erro aleatório com forma conhecida. Com $y_{t} \in \mathbb{R}^{p}$ representado por uma função $g: \mathbb{R}^{n} \times \mathbb{R}^{\Rightarrow} \mathbb{R}^{p}$, tal que:

$$
y_{t}=g\left(\theta_{t}, \nu_{t}\right),
$$

com $\nu_{t} \in \mathbb{R}^{r}$ um erro aleatório com distribuição conhecida.

O objetivo do processo é obter a função de densidade de $\theta_{t}$ dada toda a informação observada até o tempo $t, p\left(\theta_{t} \mid D_{t}\right)$. Considerando conhecida a distribuição a posteriori $p\left(\theta_{t-1} \mid D_{t-1}\right)$, a priori $p\left(\theta_{t} \mid D_{t-1}\right)$ é dada por:

$$
p\left(\theta_{t} \mid D_{t-1}\right)=\int p\left(\theta_{t} \mid \theta_{t-1}\right) p\left(\theta_{t-1} \mid D_{t-1}\right) d \theta_{t-1} .
$$

Considere ainda $\theta_{t}$ independente de $\omega_{t}, \quad \forall t$, ou seja $p\left(\omega_{t} \mid \theta_{t}\right)=p\left(\omega_{t}\right)$. Com isso, temos a distribuição de $\theta_{t}$ condicional ao estado no tempo anterior $\theta_{t-1}$ é dada por:

$$
p\left(\theta_{t} \mid \theta_{t-1}\right)=\int p\left(\theta_{t} \mid \theta_{t-1}, \omega_{t-1}\right) p\left(\omega_{t-1}\right) d \omega_{t-1} .
$$

Conhecidos $\theta_{t-1}$ e $\omega_{t-1}, \theta_{t}$ é obtido de forma determinística via $f\left(\theta_{t-1}, \omega_{t-1}\right)$. Com isso, podemos reescrever a integração acima por

$$
p\left(\theta_{t} \mid \theta_{t-1}\right)=\int \delta\left(\theta_{t}-f\left(\theta_{t-1}, \omega_{t-1}\right)\right) p\left(\omega_{t-1}\right) d \omega_{t-1},
$$

com $\delta$ correspondente a função delta de Dirac, que pode ser interpretada como uma função de probabilidade degenerada no ponto $\theta_{t}=f\left(\theta_{t-1}, \omega_{t-1}\right), \operatorname{Pr}\left(\theta_{t}=f\left(\theta_{t-1}, \omega_{t-1}\right)\right)=1$. Aplicando a integração 5.2 em 5.1, temos

$$
p\left(\theta_{t} \mid D_{t-1}\right)=\int\left[\int p\left(\theta_{t} \mid \theta_{t-1}, \omega_{t-1}\right) p\left(\omega_{t-1}\right) d \omega_{t-1}\right] p\left(\theta_{t-1} \mid D_{t-1}\right) d \theta_{t-1} .
$$

Desta forma, as amostras de $p\left(\theta_{t} \mid D_{t-1}\right)$ são obtidas a partir do passos: 
- amostrar $\theta_{t-1}$ da posteriori $p\left(\theta_{t-1} \mid D_{t-1}\right)$;

- amostrar $\omega_{t-1}$ da posteriori $p\left(\omega_{t-1}\right)$;

- obter $\theta_{t}$, por $\theta_{t}=f\left(\theta_{t-1}, \omega_{t-1}\right)$.

A intenção é varrer todo o espaço paramétrico de $\theta_{t-1}$ e $\omega_{t-1}$ por meio destas amostras, como seria desenvolvido analiticamente por meio da integração

$$
p\left(\theta_{t} \mid D_{t-1}\right)=\iint p\left(\theta_{t} \mid \theta_{t-1}, \omega_{t-1}\right) p\left(\omega_{t-1}\right) p\left(\theta_{t-1} \mid D_{t-1}\right) d \omega_{t-1} d \theta_{t-1} .
$$

Por meio do teorema de Bayes, temos:

$$
\begin{aligned}
p\left(\theta_{t} \mid D_{t}\right) & =\frac{p\left(\theta_{t}, D_{t}\right)}{p\left(D_{t}\right)}=\frac{p\left(\theta_{t}, D_{t-1}, y_{t}\right)}{p\left(D_{t-1}, y_{t}\right)} \\
& =\frac{p\left(y_{t} \mid \theta_{t}, D_{t-1}\right) p\left(\theta_{t} \mid D_{t-1}\right) p\left(D_{t-1}\right)}{p\left(y_{t} \mid D_{t-1}\right) p\left(D_{t-1}\right)} \\
& =\frac{p\left(y_{t} \mid \theta_{t}, D_{t-1}\right) p\left(\theta_{t} \mid D_{t-1}\right)}{p\left(y_{t} \mid D_{t-1}\right)} \\
& =\frac{h\left(y_{t} \mid \theta_{t}\right) p\left(\theta_{t} \mid D_{t-1}\right)}{p\left(y_{t} \mid D_{t-1}\right)}
\end{aligned}
$$

$\operatorname{com} p\left(y_{t} \mid D_{t-1}\right)$ como constante normalizadora de $g\left(y_{t} \mid \theta_{t}\right)$,

$$
p\left(y_{t} \mid D_{t-1}\right)=\int h\left(y_{t} \mid \theta_{t}\right) p\left(\theta_{t} \mid D_{t-1}\right) d \theta_{t} .
$$

Ou seja,

$$
p\left(\theta_{t} \mid D_{t}\right)=\frac{h\left(y_{t} \mid \theta_{t}\right) p\left(\theta_{t} \mid D_{t-1}\right)}{\int h\left(y_{t} \mid \theta_{t}\right) p\left(\theta_{t} \mid D_{t-1}\right) d \theta_{t}}
$$

onde

$$
h\left(y_{t} \mid \theta_{t}\right)=\int \delta\left(y_{t}-g\left(\theta_{t}, \nu_{t}\right)\right) p\left(\nu_{t}\right) d \nu_{t}
$$

Este procedimento permite a solução do processo em casos mais gerais em que $f($.$) e g($.$) não são$ necessariamente funções lineares e $\omega_{t}$ e $\nu_{t}$ não são necessariamente erros com distribuição normal.

\subsection{Tipos de filtro}

\subsubsection{Filtro de particulas bootstrap}

Gordon et al. (1993) apresentou o filtro atualmente conhecido como filtro de partículas bootstrap. Este filtro foi construído com base na amostragem por importância e reamostragem (SIR) ponderados pela função de verossimilhança. A metodologia consiste em basicamente dois passos, a propagação por meio de amostras da distribuição a priori $p\left(\theta_{t} \mid D_{t-1}\right)$ e o passo de atualização em que são reponderadas as amostras do passo anterior a fim de obter uma amostra da posteriori $p\left(\theta_{t} \mid D_{t}\right)$.

Considerando uma amostra $\left\{\theta_{t-1}^{i}, i=1, \ldots, N\right\}$ obtida da posteriori $p\left(\theta_{t-1} \mid D_{t-1}\right)$, o algoritmo do filtro bootstrap pode ser resumido da forma:

1. Propagação: obter amostras da priori $p\left(\theta_{t} \mid D_{t-1}\right)$ condicionalmente a amostra $\left\{\theta_{t-1}^{i}, i=\right.$ $1, \ldots, N\}$.

2. Atualização: utilizar as amostras obtidas no passo 1 para obter amostras da posteriori $p\left(\theta_{t} \mid D_{t}\right)$. 
O passo 1 utiliza o par $\left(\theta_{t-1}^{i}, \omega_{t-1}^{i}\right)$ para calcular os candidatos $\theta_{t}^{* i}=f\left(\theta_{t-1}^{i}, \omega_{t-1}^{i}\right), \quad i=$ $1, \ldots, N$. No passo 2 a informação de $y_{t}$ é incorporada por meio de (5.3). Temos os pesos proporcionais a verossimilhança,

$$
w_{t} \propto \frac{h\left(y_{t} \mid \theta_{t}\right)}{\int h\left(y_{t} \mid \theta_{t}\right) p\left(\theta_{t} \mid D_{t-1}\right) d \theta_{t}},
$$

Que podem ser aproximadas por

$$
w_{t}^{i}=\frac{h\left(y_{t} \mid \theta_{t}^{* i}\right)}{\sum_{j=1}^{N} h\left(y_{t} \mid \theta_{t}^{* j}\right)},
$$

$\operatorname{com} \theta_{t}^{* i} \sim p\left(\theta_{t} \mid D_{t-1}\right)$.

Smith e Gelfand (1992) sugere que para o filtro bootstrap, ao obter $\left\{\theta_{t}^{* i}, i=1, \ldots, N\right\}$ por meio de $p\left(\theta_{t}^{i} \mid D_{t-1}\right)$ e criar a função discreta tal que $p() \theta_{t}^{i}=\theta_{t}^{* i}=w_{t}^{i}$, deve-se obter posteriormente uma amostra de tamanho $\mathrm{N}$ com reposição a partir desta distribuição discreta para obter $\left\{\theta_{t}^{i}, i=\right.$ $1, \ldots, N\}$.

O passo de propagação é claramente justificado, uma vez que $\theta_{t}^{* i}$ é obtido de forma determinística por meio de $f\left(\theta_{t-1}^{i}, \omega_{t-1}^{i}\right)$, com os valores conhecidos de $\theta_{t-1}^{i}$ e $\omega_{t-}^{i}$. A justificativa da atualização é dada via teorema de Bayes aplicado via bootstrap (Smith e Gelfand, 1992). O algoritmo do filtro bootstrap é dado por:

1. Para $t=1$, gerar $\left\{\theta_{0}^{i}, i=1, \ldots, N\right\} \sim p\left(\theta_{0}\right)$;

2. Para $t>1$, utilizar $\left\{\theta_{t-1}^{i}, i=1, \ldots, N\right\}$;

gerar amostras para $\omega_{t}^{i} \sim p\left(\omega_{t}\right)$;

obter $\theta_{t}^{* i}$ de forma determinística, $\theta_{t}^{* i}=f\left(\theta_{t-1}^{i}, \omega_{t}^{i}\right)$;

obter $\nu_{t} \sim p\left(\nu_{t}\right)$ e atualizar $\theta_{t-1}^{* i}$ ponderado por

$$
w_{t}^{i}=\frac{p\left(y_{t} \mid \theta_{t}^{* i}, \nu_{t}\right.}{\sum_{j=1}^{N} p\left(y_{t} \mid \theta_{t}^{* i}, \nu_{t}\right)} ;
$$

3. Reamostrar $\left\{\theta_{t}^{* i}, i=1, \ldots, N\right\}$, de tamanho $N$ com reposição com probabilidade $w_{t}$.

\subsubsection{Filtro de partículas auxiliar}

Este filtro foi apresentado por Pitt e Shephard (1999) e tem como principal vantagem a geração de partículas com grande massa de probabilidade, devido ao aproveitamento informações da parte observável do sistema para gerar propostas mais consistentes para os estados latentes.

Assim como no filtro bootstrap, estamos interessados em obter amostras de $p\left(\theta_{t} \mid D_{t}\right)$, que são as partículas $\left(\theta_{t}^{1}, \ldots, \theta_{t}^{N}\right)$ que possuem densidade $\left(\pi_{t}^{1}, \ldots, \pi_{t}^{N}\right)$. Desta forma, partindo da distribuição a priori

$$
p\left(\theta_{t} \mid D_{t-1}\right)=\int p\left(\theta_{t} \mid \theta_{t-1}\right) p\left(\theta_{t-1} \mid D_{t-1}\right) \theta_{t-1}
$$

que pode ser aproximada por

$$
\hat{p}\left(\theta_{t} \mid D_{t-1}\right) \approx \sum_{i=1}^{N} p\left(\theta_{t} \mid \theta_{t-1}^{i}\right) \pi_{t-1}^{i},
$$

$\operatorname{com} \pi_{t-1}^{i}=p\left(\theta_{t-1}=\theta_{t-1}^{i} \mid D_{t-1}\right)$. Podemos obter a posteriori por

$$
\hat{p}\left(\theta_{t} \mid D_{t}\right) \propto p\left(y_{t} \mid D_{t}\right) \sum_{i=1}^{N} p\left(\theta_{t} \mid \theta_{t-1}^{i}\right) \pi_{t-1}^{i} .
$$


A partir da distribuição proposta gera-se novas partículas $\left(\theta_{t}^{1}, \ldots, \theta_{t}^{N}\right)$ com densidades $\left(\pi_{t}^{1}, \ldots, \pi_{t}^{N}\right)$. Dentre as técnicas para obter amostras de $\hat{p}\left(\theta_{t} \mid D_{t}\right)$, SIR, apresentada por Rubin (1992) é a mais utilizada. No entanto, Pitt e Shephard (1999) mostra que o SIR pode obter amostras muito imprecisas na medida em que $\pi_{t}$ se torna muito variável. Isto ocorre quando a verossimilhança $f(y \mid \theta)$ é muito leptocúrtica em relação a priori $f(\theta)$, isto é, apresenta caudas mais pesadas.

Uma forma de corrigir este problema é o uso de SIR adaptativo em que as propostas utilizam as informações de $y$, pare que não sejam feitas "às cegas" ("blindly") como no caso do SIR usual. O Filtro de partículas é chamado de filtro adaptativo quando há a possibilidade de inserir o conhecimento da observação $y$, conforme a estrutura a seguir

- amostra-se $\left(\theta^{1}, \ldots, \theta^{N}\right) \sim p(\theta \mid y)$

- calcula-se $w^{i}=\frac{f\left(y \mid \theta^{i}\right) f\left(\theta^{i}\right)}{p(\theta \mid y)}, i=1, \ldots, N$;

- Reamostra-se $\theta^{i}$ com os pesos $w_{i}$ para obter amostras de tamanho M.

Isto implica no cálculo de $N \times M$ densidades, ou seja a adaptação eleva o custo computacional do filtro.

É possível reduzir o alto custo computacional quando a análise via distribuições marginais é trocada por uma análise via distribuições conjuntas das variáveis envolvidas no processo (Pitt e Shephard, 1999).

A ideia do filtro de partículas auxiliar é amostrar a partir da distribuição conjunta $p\left(\theta_{t-1}, k \mid D_{t-1}\right)$,

$$
p\left(\theta_{t}, k \mid D_{t}\right) \propto p\left(y_{t} \mid \theta_{t}\right) p\left(\theta_{t} \mid \theta_{t-1}^{k}\right) \pi^{k}, \quad k=1, \ldots, M,
$$

sendo $k$ um índice referente ao elemento da amostra. Temos que,

$$
p\left(\theta_{t} \mid D_{t}\right)=\int p\left(\theta_{t}, k \mid D_{t}\right) d k
$$

em que

$$
\begin{aligned}
p\left(\theta_{t}, k \mid D_{t}\right) & =\frac{p\left(y_{t} \mid \theta_{t}, k, D_{t-1}\right) p\left(\theta_{t}, k \mid D_{t-1}\right)}{p\left(y_{t} \mid D_{t-1}\right)} \\
& =\frac{p\left(y_{t} \mid \theta_{t}\right) p\left(\theta_{t}, k \mid D_{t-1}\right)}{p\left(y_{t} \mid D_{t-1}\right)}
\end{aligned}
$$

com

$$
p\left(\theta_{t}, k \mid D_{t-1}\right)=\int p\left(\theta_{t} \mid \theta_{t-1}\right) p\left(\theta_{t-1}, k \mid D_{t-1}\right) d \theta_{t-1}
$$

e

$$
p\left(y_{t} \mid D_{t-1}\right)=\iint p\left(y_{t} \mid \theta_{t}\right) p\left(\theta_{t}, k \mid D_{t-1}\right) d k d \theta_{t} .
$$

Para a integração em 5.4 usamos

$$
p\left(\theta_{t-1}, k \mid D_{t-1}\right) \approx p\left(\theta_{t-1}=\theta_{t-1}^{k} \mid D_{t-1}\right)=\pi^{k} .
$$

Isto é, para cada valor de $\theta_{t-1}^{k}$, tem-se:

$$
p\left(\theta_{t}, k \mid D_{t}\right) \propto p\left(y_{t} \mid \theta_{t}\right) p\left(\theta_{t} \mid \theta_{t-1}^{k}\right) \pi^{k}, \quad k=1, \ldots, M .
$$

Portanto, com base na expressão (5.5), amostra-se partículas para estimar a posteriori por meio de uma variável auxiliar (índice) $k$.

A variável $k$ é chamada de auxiliar, uma vez que ela está presente apenas para auxiliar na tarefa de simulação e geração das partículas. Pode-se amostrar de $p\left(\theta_{t}, k \mid D_{t-1}\right)$ utilizando a técnica de amostragem por importância com reamostragem (SIR). A idéia é obter $N$ amostras para $\left(\theta_{t}^{i}, k^{i}\right) \sim$ 
$p\left(\theta_{t}, k \mid D_{t-1}\right)$ e reconstruir os pesos:

$$
w_{i}=\frac{p\left(y_{t} \mid \theta_{t}^{i}\right) p\left(\theta_{t}^{i} \mid \theta_{t-1}^{k^{i}}\right)}{p\left(\theta_{t}^{i}, k^{i} \mid D_{t}\right)}, \quad \pi_{i}=\frac{w_{i}}{\sum_{j=1}^{N} w_{j}}, i=1, \ldots, N
$$

Cabe ressaltar queo uso da variável auxiliar não é o suficente para considerar o filtro um processo não "às cegas" (Pitt e Shephard, 1999). Tem-se total controle do delineamento da distribuição, que pode depender tanto de $y_{t}$ como de $\theta_{t-1}^{k}$. Pode-se aproximar $\hat{p}\left(\theta_{t} \mid D_{t}\right)$ por:

$$
p\left(\theta_{t}, k \mid D_{t}\right) \propto p\left(y_{t} \mid \mu_{t}^{k}\right) p\left(\theta_{t} \mid \theta_{t-1}^{k}\right) \pi^{k}, \quad k=1, \ldots, M,
$$

com $\mu_{t}^{k}$ uma medida representativa de $p\left(\theta_{t} \mid \theta_{t-1}^{k}\right)$, esta pode ser uma média, moda, mediana ou uma amostra de $p\left(\theta_{t}^{i} \mid \theta_{t-1}^{k}\right)$.

A densidade marginal em relação a $k$ é dada por:

$$
\begin{aligned}
p\left(k \mid D_{t}\right) & =\int p\left(\theta_{t}, k \mid D_{t}\right) d \theta_{t} \\
& \propto \pi^{k} \int p\left(y_{t} \mid \mu_{t}^{k}\right) p\left(\theta_{t} \mid \theta_{t-1}^{k}\right) d \theta_{t} \\
& \propto \pi^{k} \int p\left(y_{t} \mid \mu_{t}^{k}\right)
\end{aligned}
$$

A amostra de $p\left(\theta_{t}, k \mid D_{t}\right)$ é simulada amostrando, primeiramente, o índice $k$, com probabilidade $\lambda_{k} \sim p\left(k \mid D_{t}\right)$. Em seguida, amostra-se $\theta_{t}$ a partir de $p\left(\theta_{t}^{i} \mid \theta_{t-1}^{k}\right)$. O peso $\lambda_{k}$ é o primeiro estágio de ponderação. Tendo amostrado $N$ vezes a densidade conjunta $p\left(\theta_{t}, k \mid D_{t}\right)$, as partículas $\left(\theta_{t}^{i}, k^{i}\right)$ são reponderadas pelos pesos

$$
w_{i}=\frac{p\left(y_{t} \mid \theta_{t}^{i}\right)}{p\left(y_{t} \mid \mu_{t}^{k^{j}}\right)}, \quad \pi_{i}=\frac{w_{i}}{\sum_{j=1}^{N} w_{j}}, j=1, \ldots, N
$$

Este procedimente permite que as propostas tenham alta verossimilhança condicional, reduzindo o desperdício em produzir partículas com baixa massa de probabilidade, ou seja, baixa representatividade na distribuição a posteriori.

O algoritmo pode ser resumido da forma:

1. Para $t=1$ obter propostas de $\theta_{1}^{*} \sim p\left(\theta_{1} \mid \theta_{0}\right)$;

2. Para $t>1$ obter propostas de $\theta_{t}^{*} \sim p\left(\theta_{t} \mid \theta_{t-1}^{k}\right)$;

3. obter valores representativos da medida $\mu^{k} \leftarrow \theta_{t}^{*} \mid \theta_{t-1}^{k}$

4. calcular os pesos $\lambda_{k} \propto w_{t-1}^{k} p\left(y_{t} \mid \mu_{t}^{k}\right)$;

5. reamostrar $N$ partículas com pesos $\lambda_{k}: \theta_{t-1}^{k^{*}} \sim\left(\lambda_{k}, \theta_{t-1}^{k}\right)$;

6. obter propostas $\theta_{t}^{i} \mid \theta_{t-1}^{k^{*}} \sim p\left(\theta_{t} \mid \theta_{t-1}^{k^{*}}\right)$;

7. obter valores representativos da medida $\mu_{t}^{k^{*}} \leftarrow \theta_{t}^{i} \mid \theta_{t-1}^{k^{*}}$;

8. atualizar os pesos das partículas $w_{i}=p\left(y_{t} \mid \theta_{t}^{i}\right) / p\left(y_{t} \mid \mu_{t}^{k^{*}}\right)$;

9. normalizar os pesos $\pi_{t}^{i}=w_{t}^{i} / \sum_{j=1}^{N} w_{t}^{j}$;

10. reamostrar $M$ partículas $\theta_{t}^{k}$ de $\theta_{t}^{i} \operatorname{com}$ peso $\pi_{t}^{i}$;

11. normalizar os pesos de $\theta_{t}^{k}, \pi_{t}^{k}=w_{t}^{k} / \sum_{j=1}^{M} w_{t}^{j}$. 


\subsection{Estimação dos parâmetros}

Em grande parte dos casos de modelos dinâmicos, há presença de parâmetros fixos desconhecidos, nesta seção apresentamos algumas alternativas para estimação sequencial dos parâmetros estaticos do modelo.

\subsubsection{Filtro Liu e West}

Liu e West (2001) sugere uma metodologia de estimação dos parâmetros por meio de uma suavização via kernel (West, 1993a; West, 1993b) combinado com o filtro de partículas auxiliar.

Seja a série temporal $\left\{y_{t}: t=1, \ldots, n\right\}$ e a modelagem via filtro de partículas com respectivamente o vetor de espaços latentes e o vetor de parâmetros desconhecidos $\left\{x_{t}, \theta_{t}: t=1, \ldots, n\right\}$ associados ao peso $\left\{w_{t}: t=1, \ldots, n\right\}$. A partir deste conjunto de partículas, aproxima-se a distribuição a posteriori no tempo $t, p\left(x_{t}, \theta \mid D_{t}\right)$. Para o processo de estimação a posteriori conjunta no tempo $t+1$, temos

$$
\begin{aligned}
p\left(x_{t+1}, \theta \mid D_{t+1}\right) & =p\left(y_{t+1} \mid x_{t+1}, \theta\right) p\left(x_{t+1}, \theta \mid D_{t}\right) \\
& =p\left(y_{t+1} \mid x_{t+1}, \theta\right) p\left(x_{t+1} \mid \theta, D_{t}\right) p\left(\theta \mid D_{t}\right) .
\end{aligned}
$$

Para o processo numérico de estimação, considere o uso de $\theta_{t}$ no lugar $\theta$. Apesar $\theta_{t}$ representar um vetor de parâmetros estáticos, esta representação auxilia no processo de estimação ao longo do tempo. Considere a seguinte representação artificial da evolução de $t$ para $t+1$ das estimativas,

$$
\theta_{t+1}=\theta_{t}+\epsilon_{t+1},
$$

com $\epsilon_{t+1} \sim N(0, H)$, sendo $H$ uma matriz de variância e $\theta_{t}$ e $\epsilon_{t+1}$ condicionalmente independentes a $D_{t}$. Desta forma, a densidade a posteriori $p\left(\theta \mid D_{t}\right)$ é feita por densidade suavizade de kernel,

$$
p\left(\theta \mid D_{t}\right) \approx \sum_{j=1}^{N} w_{t}^{j} N\left(\theta \mid m_{t}^{i}, h^{2} V_{t}\right) .
$$

Assim, a distribuição artificial dos parâmetros $\theta$ é representada pela mistura ponderada por $w_{t}^{i}$ de normais com média $m_{t}^{i}$ e matriz de variância $h^{2} V_{t}$. Liu e West (2001) sugerem que a constante $h$ seja escolhida como uma função suave decrescente de $N$. Liu e West (2001) propoem:

$$
\begin{aligned}
m_{t}^{j} & =a \theta_{t}^{j}+(1-a) \hat{\theta}_{t}, \\
a & =\sqrt{\left(1-h^{2}\right)}, \\
\hat{\theta}_{t} & =\sum_{i=1}^{N} \frac{\theta_{t}^{i}}{N}, \\
V_{t} & =\frac{1}{N} \sum_{i=1}^{N}\left(\theta_{t}^{i}-\hat{\theta}_{t}\right)\left(\theta_{t}^{i}-\hat{\theta}_{t}\right)^{\prime} .
\end{aligned}
$$

As constantes $a$ e $h$ medem o encolhimento e superdispersão da mistura, respectivamente.

$\mathrm{O}$ algoritmo pode ser sumarizado da seguinte forma:

1. identificar os pontos

$$
\begin{aligned}
\mu_{t}^{i} & =E\left(x_{t} \mid x_{t-1}^{i}, \theta\right) \\
m_{t-1}^{i} & =a \theta_{t-1}^{i}+(1-a) \hat{\theta}_{t-1}
\end{aligned}
$$


2. amostrar variável auxiliar $k^{i}$ com probabilidade proporcional a

$$
p\left(k^{i}=k\right) \propto w_{t-1}^{i} p\left(y_{t} \mid \mu_{t}^{i}, m_{t-1}^{i}\right)
$$

3. Amostrar o vetor de parâmetros $\theta_{t}^{i}$

$$
\theta_{t}^{i} \sim N\left(m_{t-1}^{k^{i}}, h^{2} V_{t-1}\right),
$$

$\operatorname{com} V_{t}=\sum_{i=1}^{N}\left(\theta_{t-1}^{i}-\hat{\theta}_{t-1}\right)\left(\theta_{t-1}^{i}-\hat{\theta}_{t-1}\right)^{\prime} w_{t-1}^{i}$.

4. Obter uma amostra $x_{t}^{i}$ da variável latente a partir de $p\left(x_{t} \mid x_{t-1}^{k^{i}}, \theta_{t}^{i}\right)$;

5. Calcular os pesos

$$
w_{t}^{i} \propto \frac{p\left(y_{t} \mid x_{t}^{i}, \theta_{t}^{i}\right)}{p\left(y_{t} \mid \mu_{t}^{k^{i}}, \theta_{t}^{k^{i}}\right)}
$$

6. obter $p\left(x_{t}, \theta \mid D_{t}\right)$ a partir de $\left\{\left(x_{t}, \theta_{t}, w_{t}\right)^{i}, i=1, \ldots, N\right\}$.

\subsubsection{Filtro Storvik}

Storvik (2002) propõe o uso de um conjunto de estatísticas suficientes para calcular a posteriori dos parâmetros estáticos $\theta$, condicionados as observações $y_{t}$ e aos espaços latentes $x_{t}$ e que são atualizados recursivamente. Desta forma, o processo de estimação dos estados latentes passa a ter um novo passo, de atualização do vetor de parâmetros.

A metodologia de Storvik (2002) pode ser utilizada quando se conhece as distribuições marginais dos parâmetros a posteriori. As distribuições são calculadas de forma semelhande a quando se tem forma fechada, descrita por West e Harrison (1997), no entanto as posterioris são calculadas até o tempo presente $t$, ao invés de calcular somente após observar toda a série.

A técnica utilizada na atualização é conhecida como Rao-Blackwellization (Doucet et al., 2004). Considere $S_{t}^{i}=\left\{S\left(x_{1: t}^{i}, D_{t}^{i}\right)\right\}$ o conjunto de estatísticas suficientes para a distribuição a posteriori de $\theta_{t}^{i}$, onde:

$$
w_{t}=\frac{p\left(x_{0: t} \mid D_{t}\right)}{p\left(x_{0}\right) \prod j=1^{t} p\left(x_{j} \mid x_{j-1}, y_{j}\right)}
$$

e ainda,

$$
\hat{p}\left(x_{1: t-1} \mid D_{t-1}\right)=\frac{\sum_{j=1}^{N} w_{t-1}^{j} I\left(x_{1: t-1}=x_{1: t-1}^{i}\right)}{\sum_{l=1}^{N} w_{t-1}^{l}} .
$$

Sem a presença de parâmetros estáticos desconhecidos, a distribuição a posteriori é dada por:

$$
\begin{aligned}
p\left(x_{1: t} \mid D_{t}\right) & \propto p\left(x_{1: t-1} \mid D_{t-1}\right) p\left(x_{t} \mid x_{1: t-1}\right) p\left(y_{t} \mid x_{t}\right) \\
& =\hat{p}\left(x_{1: t-1} \mid D_{t-1}\right) p\left(x_{t} \mid x_{1: t-1}\right) p\left(y_{t} \mid x_{t}\right) .
\end{aligned}
$$

Um novo conjunto de partículas pode ser obtido ao simular desta distribuição aproximada. Um procedimento de rejeição possível para simular dados a partir de (5.6) consiste em amostrar $x_{1: t}$ a partir de $\hat{p}\left(x_{1: t} \mid D_{t-1}\right)=\hat{p}\left(x_{1: t-1} \mid D_{t-1}\right) p\left(x_{t} \mid x_{t-1}\right)$ e aceitar a amostra com probabilidade proporcional a $p\left(y_{t} \mid x_{t}\right)$.

Na presença de parâmetros estáticos desconhecidos, um novo processo é adicionado referente a posteriori relativa $\theta$. Storvik (2002) sugere que a estimativa de $\theta$ no tempo $t$ dependa apenas do conjunto de estatísticas suficientes $S_{t}=S\left(x_{1: t}, D_{t}\right)$ que são atualizadas a cada tempo $t$. Considere ainda,

$$
p\left(x_{1: t}, \theta \mid D_{t}\right) \propto p\left(x_{1: t-1} \mid D_{t-1}\right) p\left(\theta \mid x_{1: t-1}, D_{t-1}\right) p\left(y_{t} \mid x_{t}, \theta\right) .
$$

As simulações a partir de (5.7) podem ser realizadas ao simular $x_{1: t-1}$ a partir de $\hat{p}\left(x_{1: t-1} \mid D_{t-1}\right)$, $\theta$ por $p\left(\theta \mid S_{t}\right), x_{t}$ a partir de $p\left(x_{t} \mid x_{1: t-1}, \theta\right)$ e aceitar com probabilidade proporcional a $p\left(y_{t} \mid x_{t}, \theta\right)$. 
Cabe ressaltar que a distribuição de atualização de $\theta, p\left(\theta \mid x_{1: t}, D_{t}\right)$ depende de $\left(x_{1: t}, y 1: t\right)$ somente por meio das estatísticas suficientes $S_{t}$, e com isso se tem uma redução na dimensão e na complexidade do processo de estimação. Em resumo, o algoritmo é dado por:

1. $\operatorname{amostrar} \theta \sim p\left(\theta \mid x_{1: t-1}^{i}, D_{t}\right)$;

2. $\operatorname{amostrar} x_{t}^{* i} \sim p\left(x_{t} \mid x_{1: t-1}^{i}, y_{t}, \theta\right)$

3. obter os pesos

$$
w_{t}^{i} \propto w_{t-1}^{i} \frac{p\left(\theta \mid S_{t}^{i}\right) p\left(x_{t}^{* i} \mid x_{1: t-1}^{i}, \theta\right) p\left(y_{t} \mid x_{t}^{* i}, \theta\right)}{p\left(\theta \mid x_{1: t-1}^{i}, D_{t}\right) p\left(x_{t} \mid x_{t-1}^{i}, y_{t}, \theta\right)} .
$$

4. Reamostrar as partículas $x_{t}^{* i}$ com peso $w_{t}^{i}$.

As distribuições $p\left(\theta \mid x_{1: t-1}^{i}, D_{t}\right)$ e $p\left(x_{t} \mid x_{t-1}^{i}, y_{t}, \theta\right)$ são distribuições geradoras de candidatos de $\theta$ e $x_{t}$, respectivamente. O mais comum é utilizar $p\left(\theta \mid x_{1: t-1}^{i}, D_{t}\right)=p\left(\theta \mid S_{t-1}\right)$ e $p\left(x_{t} \mid x_{t-1}^{i}, y_{t}, \theta\right)=$ $p\left(x_{t} \mid x_{t-1}, \theta\right)$. Desta forma, temos um procedimento de filtro bootstrap.

\subsubsection{Filtro parameter learning}

Carvalho et al. (2010) e Lopes et al. (2011) sugerem a combinação do filtro auxiliar proposto por Pitt e Shephard (1999) com a metodologia de estimação dos parâmetros estáticos de Storvik (2002). Os autores mostram vantagens deste procedimento comparados aos filtros de Liu e West e Storvik. O filtro é denominado de parameter learning. Dada as mesmas condições da presença de estatísticas suficientes do filtro Storvik, definidos por $S_{t}=S\left(x_{1: t}, D_{t}\right)$, o algoritmo do parameter learning pode ser resumido por

1. Para $t=1$ obter propostas de $\theta_{1}^{*} \sim p\left(x_{1} \mid x_{0}\right)$ e $\theta \sim p_{0}(\theta)$;

2. Para $t>1$ obter propostas de $x_{t}^{*} \sim p\left(x_{t} \mid x_{t-1}^{k}\right)$ e $\theta \sim p\left(\theta \mid S_{t-1}^{k}\right)$;

3. obter valores representativos da medida $\mu^{k} \leftarrow\left(x_{t}^{*} \mid x_{t-1}^{k}, \theta\right)$

4. calcular os pesos $\lambda_{k} \propto w_{t-1}^{k} p\left(y_{t} \mid \mu_{t}^{k}, \theta^{k}\right)$;

5. reamostrar $N$ partículas com pesos $\lambda_{k}: x_{t-1}^{k^{*}} \sim\left(\lambda_{k}, x_{t-1}^{k}, \theta^{k}\right)$;

6. obter propostas $\left(x_{t}^{i} \mid x_{t-1}^{k^{*}}, \theta^{k^{*}}\right) \sim p\left(x_{t} \mid x_{t-1}^{k^{*}}, \theta^{k^{*}}\right)$;

7. obter valores representativos da medida $\mu_{t}^{k^{*}} \leftarrow\left(x_{t}^{i} \mid x_{t-1}^{k^{*}}, \theta^{k^{*}}\right)$;

8. atualizar os pesos das partículas $w_{i}=p\left(y_{t} \mid x_{t}^{i}, \theta^{k^{*}}\right) / p\left(y_{t} \mid \mu_{t}^{k^{*}}, \theta^{k^{*}}\right)$;

9. normalizar os pesos $\pi_{t}^{i}=w_{t}^{i} / \sum_{j=1}^{N} w_{t}^{j}$;

10. reamostrar $M$ partículas $x_{t}^{k}$ de $x_{t}^{i}$ com peso $\pi_{t}^{i}$;

11. atualizar $S_{t}^{k}=S\left(S_{t-1}^{k}, x_{t}^{k}, y_{t}\right)$

12. normalizar os pesos de $x_{t}^{k}, \pi_{t}^{k}=w_{t}^{k} / \sum_{j=1}^{M} w_{t}^{j}$.

\subsection{Suavização}

Ao final do processo sequencial de filtro de partículas, pode-se utilizar uma estimação "backward" a fim de se obter posterioris dos espaços de estados dado todas as observações da série, ou seja, $p\left(x_{t} \mid D_{n}\right)$, para $t=1, \ldots, n$. Este processo recebe o nome de suavização. 
Considere a decomposição da distribuição conjunta obtida ao final do processo de filtragem, $p\left(x_{1: n} \mid D_{n}\right)$.

$$
\begin{aligned}
p\left(x_{1: n} \mid D_{n}\right) & =p\left(x_{n} \mid D_{n}\right) \prod_{t=1}^{n-1} p\left(x_{t} \mid x_{t+1}, D_{n}\right) \\
& =p\left(x_{n} \mid D_{n}\right) \prod_{t=1}^{n-1} p\left(x_{t} \mid x_{t+1}, D_{t}\right) .
\end{aligned}
$$

Este resultado é garantido pelas propriedades de independência da cadeia de Markov. Por meio destas propriedades, temos: Considere a decomposição da distribuição conjunta obtida ao final do processo de filtragem, $p\left(x_{1: n} \mid D_{n}\right)$.

$$
\begin{aligned}
p\left(x_{t} \mid x_{t+1: n}, D_{n}\right) & =p\left(x_{t} \mid x_{t+1}, D_{n}\right) \\
& =\frac{p\left(x_{t+1} \mid x_{t}\right) p\left(x_{t} \mid D_{t}\right)}{p\left(x_{t+1} \mid D_{t}\right)} \\
& \propto p\left(x_{t} \mid D_{t}\right) p\left(x_{t+1} \mid x_{t}\right) .
\end{aligned}
$$

Por meio das estimativas a posteriori $\hat{p}\left(x_{t} \mid D_{t}\right)$ obtidas pela filtragem, Doucet et al. (2004) mostra que a expressão acima pode ser aproximada por

$$
p\left(x_{t} \mid x_{t+1}, D_{n}\right) \approx \sum_{i=1}^{N} w_{t \mid t+1}^{i} \delta_{x_{t}^{i}}\left(x_{t}\right),
$$

sendo $w_{t \mid t+1}^{i}$ os pesos de suavização expressos pela equação:

$$
w_{t \mid t+1}^{i}=\frac{w_{t}^{i} p\left(x_{t+1} \mid x_{t}^{i}\right)}{\sum_{j=1}^{N} w_{t}^{j} p\left(x_{t+1} \mid x_{t}^{j}\right)},
$$

onde $w_{t}^{i}$ corresponde ao peso obtido no processo on-line de filtragem.

Desta forma, pode-se obter amostras suavizadas dos estados latentes por meio de $\tilde{x}_{t} \sim \hat{p}\left(x_{t} \mid x_{t: n}, D_{n}\right)$ em um processo de "trás para frente".

Mais detalhes sobre a metodologia de filtro de partículas em modelos dinâmicos pode ser encontrada em (Nascimento, 2014, Lopes et al., 2011 e Lopes e Carvalho, 2013). 


\section{Capítulo 6}

\section{Simulação e aplicação}

Neste capítulo apresentaremos as principais contribuições desta tese, com a estimação dos modelos dinâmicos BIZU e Tobit via Filtro de Partículas. Inicialmente mostraremos alguns resultados de estimação dos modelos com diferentes estruturas de dados simulados. Por fim os modelos serão utilizados em um contexto real, é apresentada uma aplicação dos modelos dinâmicos para estimação e previsão do percentual do nível de água no sistema da cantareira. A competitividade entre modelos também será discutida neste estudo. Na seção (6.1)são apresentadas estimações de séries de dados simulados do modelo dinâmico $B I Z U$, na seção (6.2) são apresentadas estimações de série de dados simulados do modelo dinâmico Tobit. Na seção (6.3) é apresentada aplicação de dados reais para ambos os modelos dinâmicos.

\subsection{Estudo de simulação para o modelo dinâmico BIZU}

Considerando a estrutura do modelo apresentada na seção (4.2), temos os espaços de estados representados por $\boldsymbol{\theta}$ e o conjunto de parâmetros estáticos desconhecidos $(\alpha, \gamma, \boldsymbol{W})$ para serem estimados. Desta forma, temos os filtros Liu e West (LW), Storvik e Parameter Learning (PL), de modo que os dois últimos necessitam de estatísticas suficiente. Em todas as simulações deste estudo foram utilizadas o número de partículas $N=10000$.

Como foi descrito na seção (4.2), é possível obter as posterioris condicionais completas de $\boldsymbol{\Theta}=$ $(\alpha, \gamma, \boldsymbol{W})$ até um tempo presente $t$ :

$$
\begin{aligned}
\left(\alpha \mid \boldsymbol{\theta}_{1: t}, \gamma, \boldsymbol{\Phi}_{1}, \boldsymbol{y}_{1: t}\right) & \sim \operatorname{Beta}\left(a_{\alpha}+\sum_{i=1}^{t} \mathbb{1}_{\{0,1\}}\left(y_{i}\right) ; b_{\alpha}+t-\sum_{i=1}^{t} \mathbb{1}_{\{0,1\}}\left(y_{i}\right)\right) \\
\left(\gamma \mid \boldsymbol{\theta}_{1: t}, \alpha, \boldsymbol{\Phi}_{1}, \boldsymbol{y}_{1: t}\right) & \sim \operatorname{Beta}\left(a_{\gamma}+\sum_{i=1}^{t} \mathbb{1}_{\{1\}}\left(y_{i}\right) ; b_{\gamma}+\sum_{i=1}^{t} \mathbb{1}_{\{0,1\}}\left(y_{i}\right)-\sum_{i=1}^{t} \mathbb{1}_{\{1\}}\left(y_{i}\right)\right) ; \\
p\left(\boldsymbol{\psi}_{1} \mid \boldsymbol{\theta}_{1: t}, \alpha, \gamma, \boldsymbol{y}_{1: t}\right) & \sim W\left(t / 2+\nu_{1}, S S_{\theta_{1: t}}+\boldsymbol{S}_{1}\right),
\end{aligned}
$$

$\operatorname{com} S S_{\theta_{t}}=\left(\boldsymbol{\theta}_{t}-\boldsymbol{G}_{t} \boldsymbol{\theta}_{t-1}\right)\left(\boldsymbol{\theta}_{t}-\boldsymbol{G}_{t} \boldsymbol{\theta}_{t-1}\right)^{\prime}$ e $S S_{\theta_{1: t}}=\sum_{i=1}^{t} S S_{\theta_{i}}$.

Desta forma temos o seguinte conjuntos de estatísticas suficientes para o tempo $t$ necessárias para atualização das posterioris condicionais completas até o tempo presente:

$$
\left.\left(\sum_{i=1}^{t} \mathbb{1}_{\{0,1\}}\left(y_{i}\right), \sum_{i=1}^{t} \mathbb{1}_{\{1\}}\left(y_{i}\right)\right), S S_{\theta_{1: t}}\right) .
$$

Além disso, para o filtro auxiliar precisamos obter amostras $\mu_{t}^{k}$ como uma função de $p\left(\boldsymbol{\theta}_{t} \mid \boldsymbol{\theta}_{t-1}^{k}, \boldsymbol{\Theta}^{k}\right)$, $k=1, \ldots, N$. Pela estrutura linear do modelo é natural escolhermos a média, ou seja:

$$
\mu_{t}=E\left(\boldsymbol{\theta}_{t} \mid \boldsymbol{\theta}_{t-1}, \boldsymbol{\Theta}\right)
$$


Assim, podemos amostrar:

$$
\mu_{t}^{k} \sim N\left(G \boldsymbol{\theta}_{t-1}^{k}, W^{k}\right) .
$$

Tendo estas medidas definidas, para o processo de estimação via filtro de partículas basta seguir os algoritmos descritos em 5.3.

\subsubsection{Modelo de passeio aleatório}

Com o intuito de verificar o funcionamento do filtro de partículas no modelo BIZU, começamos pelo caso mais simples de um modelo dinâmico, dado pelo sistema de equações.

$$
\begin{aligned}
& \eta_{t}=\theta_{t} \\
& \theta_{t}=\theta_{t-1}+\omega, \quad \omega \sim N(0, W) .
\end{aligned}
$$

Com $\eta_{t}$ e $\theta_{t}$ vetores de dimensão 2 e $W$ uma matriz de covariância $2 \times 2$.

Como os espaços de estados latentes não estão atrelados às observações, $y_{t}$, de forma direta, e sim por meio de uma função de ligação, $\eta_{t}$, aplicada aos parâmetros de média $\mu_{t}$ e precisão $\phi_{t}$, o filtro de bootstrap proporciona grande quantidade de partículas com baixa massa de probabilidade. Com isso, o filtro bootstrap não é muito adequado para o problema. Neste estudo serão apresentados comparações entre os filtro Liu e West e Parameter Learning.

Inicialmente foi gerada uma serie de tamanho $T=84$ e foram fixados os valores de $\alpha=0,05$, representando a grau de mistura do componente discreto no modelo beta, e $\gamma=0,4$, representando o parâmetro de probabilidade da componente discreta do modelo. A variância do componente do erro da equação de evolução foi fixada em $W=\operatorname{diag}(0,04,0,0004)$. Os hiperparâmetros da priori inicial para os espaços de estados foram definidos por $m_{0}=(\operatorname{logit}(0,3), \log (200))^{\prime}$ e $C_{0}=\operatorname{diag}(0,25,1)$, $\theta_{0} \sim N\left(m_{0}, C_{0}\right)$.

Para ambos os filtros foram atribuídas as prioris: $\alpha_{0} \sim B(3,35)$ e $\gamma_{0} \sim B(10,15)$. Estes hiperparâmetros foram escolhidos afim de obter uma distribuição centrada na proporção de zeros e uns na série. As prioris para as componentes da variância do erro de evolução foram dadas por $W_{1}^{-1} \sim G(5 / 2,0,05 \times 5 / 2)$ e $W_{2}^{-1} \sim G(5 / 2,0,006 \times 5 / 2)$, com $W_{0}=\operatorname{diag}\left(W_{1}, W_{2}\right)$ e os hiperparâmetros para a priori do espaço de estado inicial foram $m_{0}=\left(\operatorname{logit}\left(y_{1}\right),-\log \left(\operatorname{var}\left(y_{1: T}\right)\right)\right)^{\prime} \mathrm{e}$ $C_{0}=\operatorname{diag}\left(0,5^{2}, 0,15^{2}\right)$.

Os filtros apresentaram desempenho semelhante para os espaços de estados. No entanto, por não utilizar nenhuma informação dos dados para as propostas dos parâmetros estáticos, a estimação via filtro PL foi ligeiramente superior ao filtro LW como se pode observar nas Figuras (6.3), (6.6), (6.4) e (6.7). O processo computacional segue os algoritmo descritos na Seção 5.3 utilizando as distribuições a posteriori dos parâmetros descritas em cada caso. O filtro LW apresentou um custo computacional inferior ao filtro PL. Enquanto o processo pelo filtro LW levou o tempo aproximado de 5 minutos, o filtro PL levou certa de 20 minutos. 


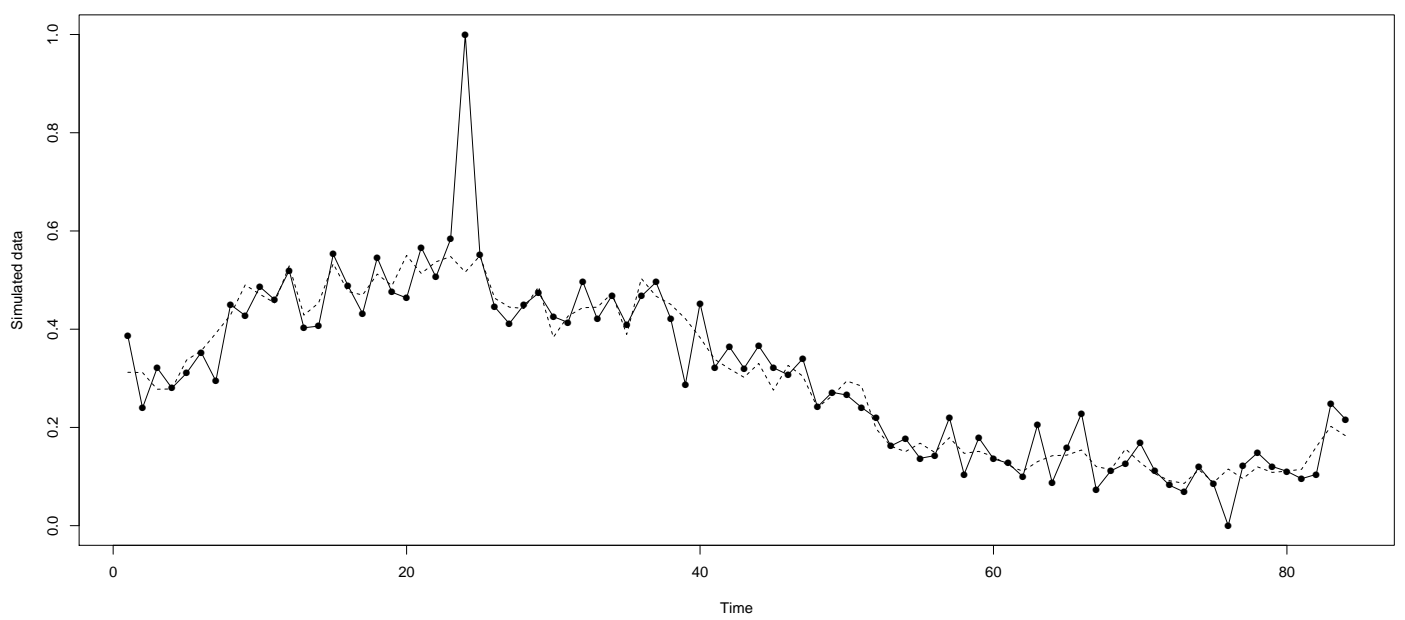

Figura 6.1: Gráficos dos dados simulados do modelo BIZU com passeio aleatório (linha contínua) e seu parâmetro $\mu_{t}$ (linha pontilhada).
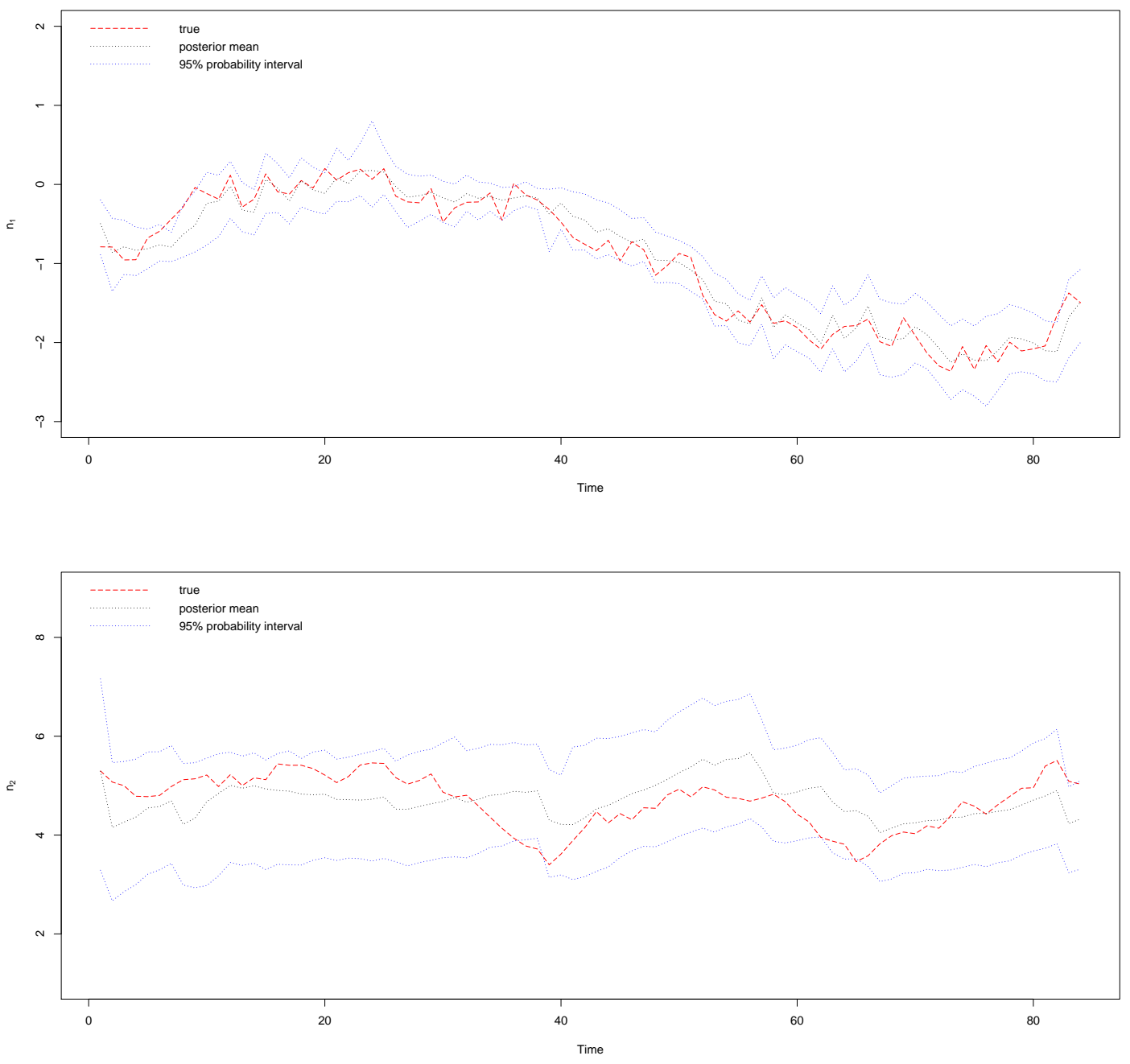

Figura 6.2: Gráficos dos preditores lineares de $\mu_{t}$ e $\phi_{t}$ do modelo BIZU estimados via filtro LW. 

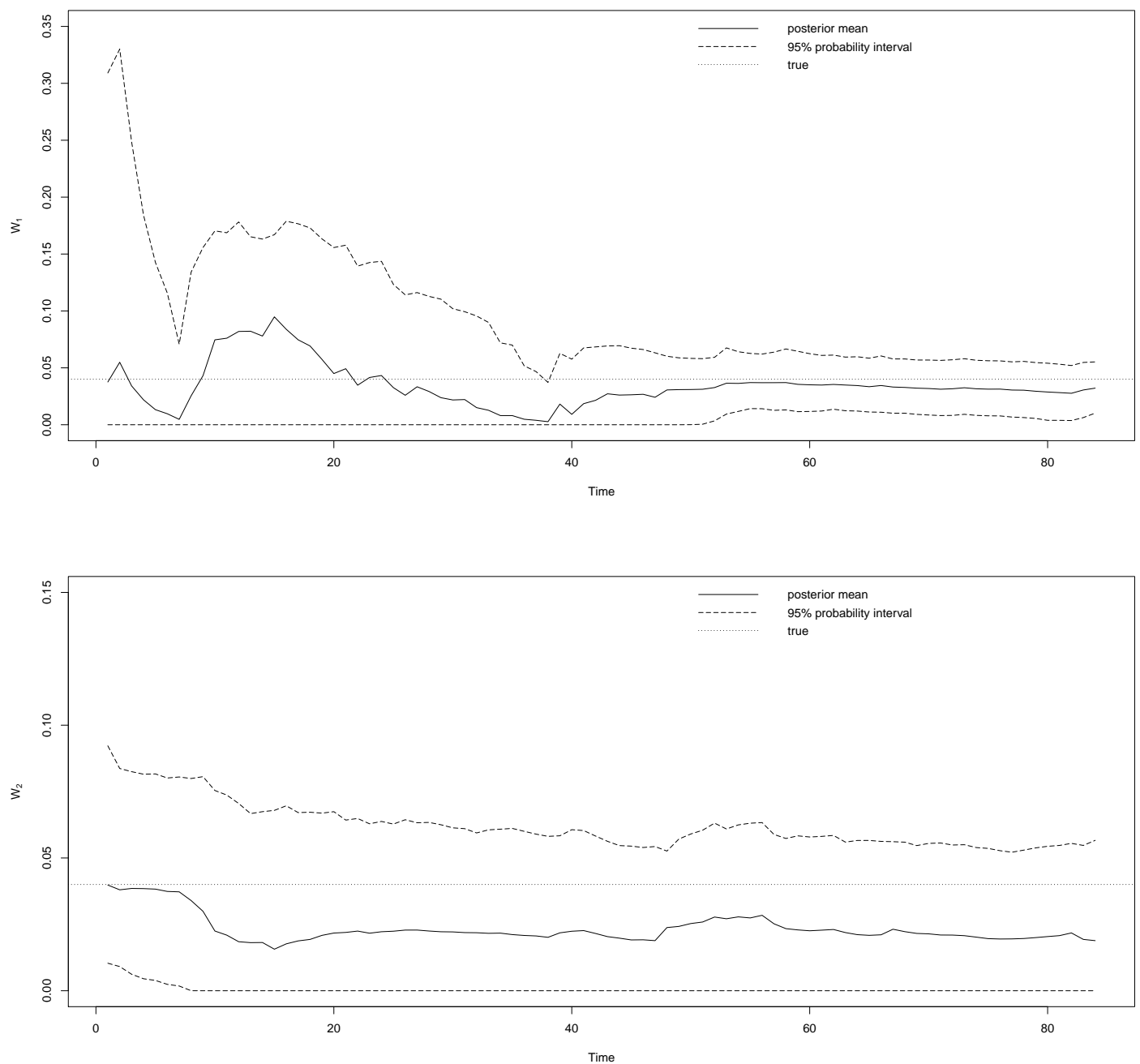

Figura 6.3: Gráfico da estimativa das componentes da variância $W$ do modelo BIZU via filtro $L W$. 

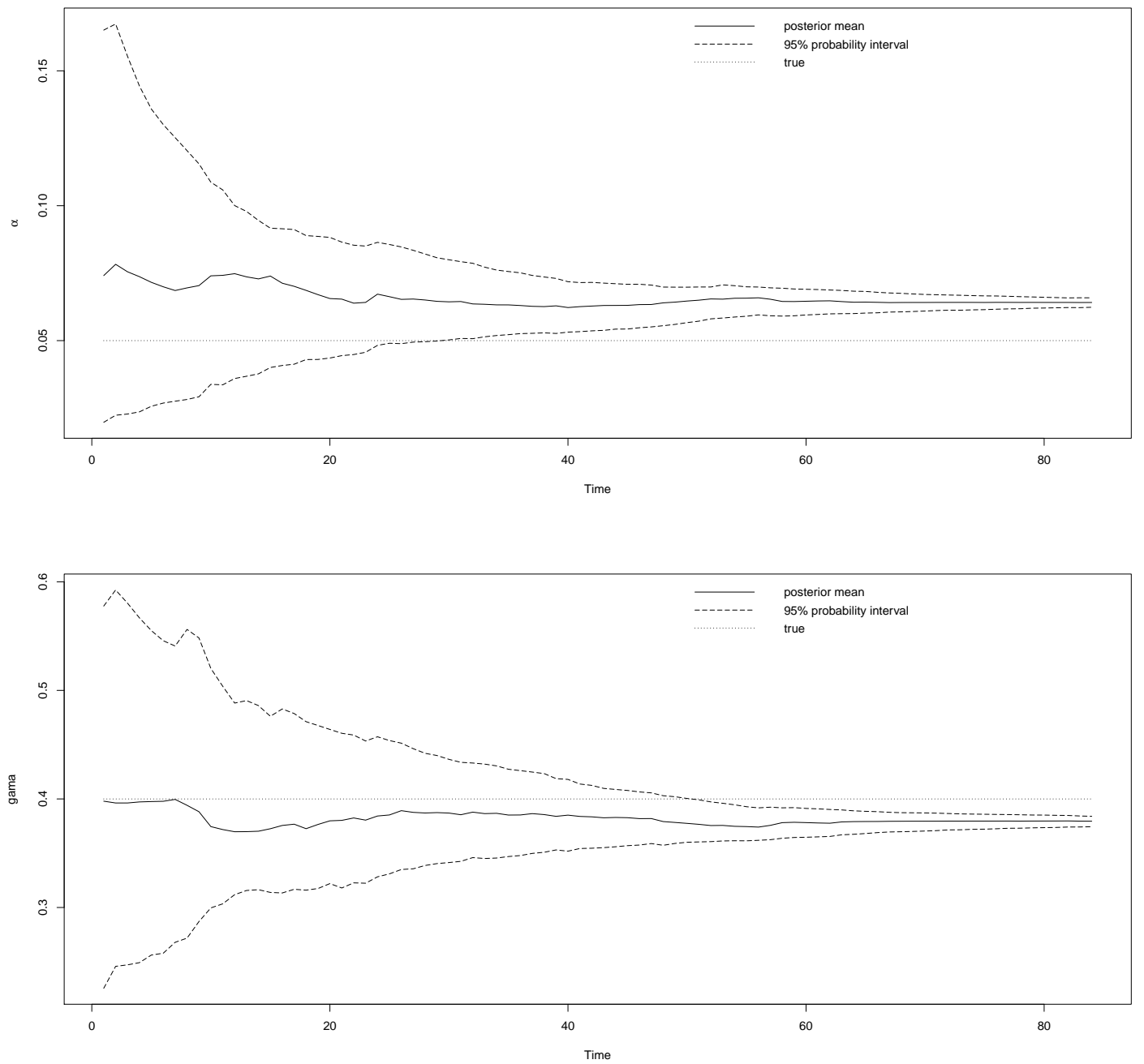

Figura 6.4: Gráfico da estimativa das probabilidades $\alpha$ e $\gamma$ do modelo BIZU via filtro $L W$. 

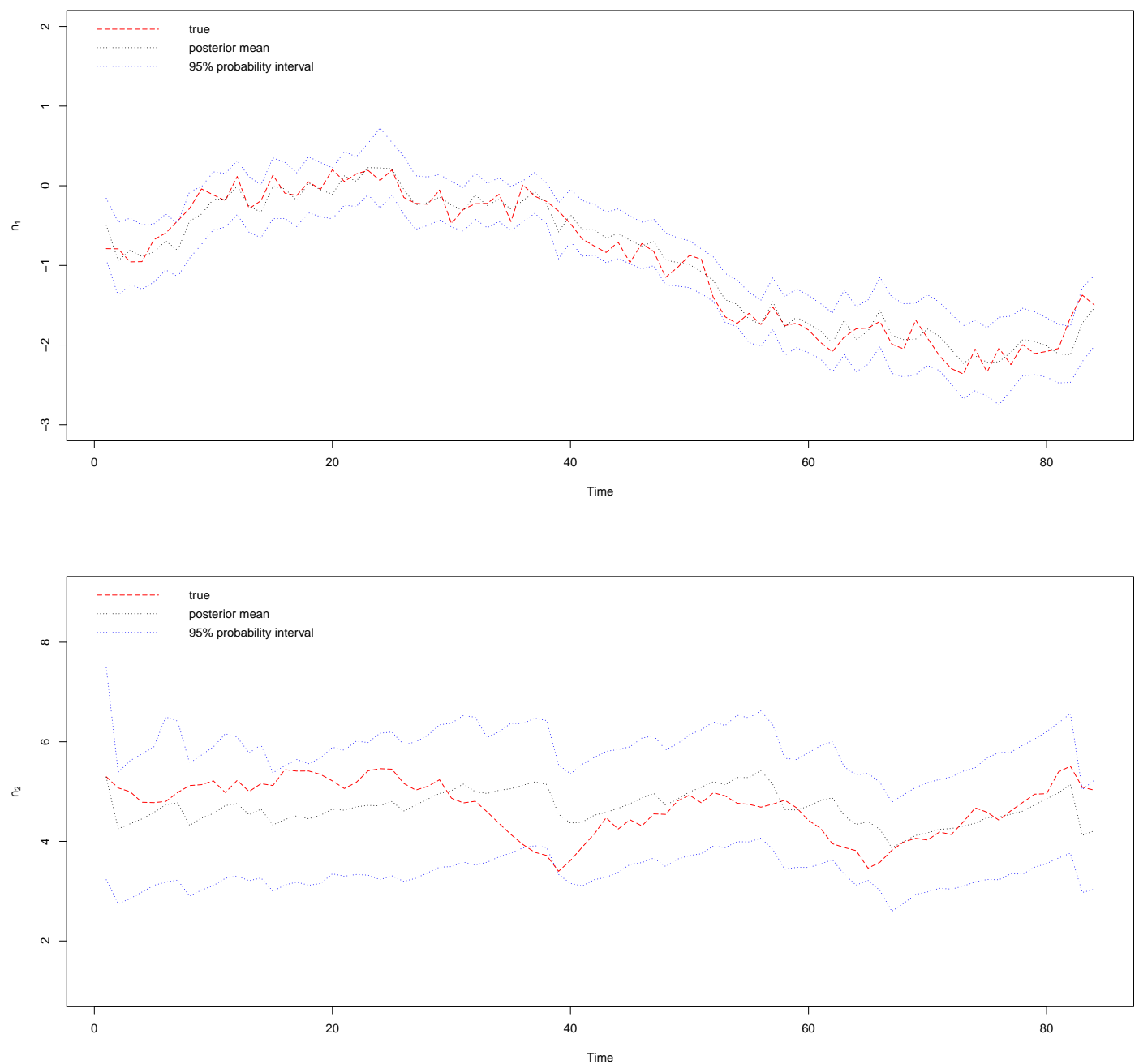

Figura 6.5: Gráficos dos preditores lineares de $\mu_{t}$ e $\phi_{t}$ do modelo BIZU estimados via filtro PL. 

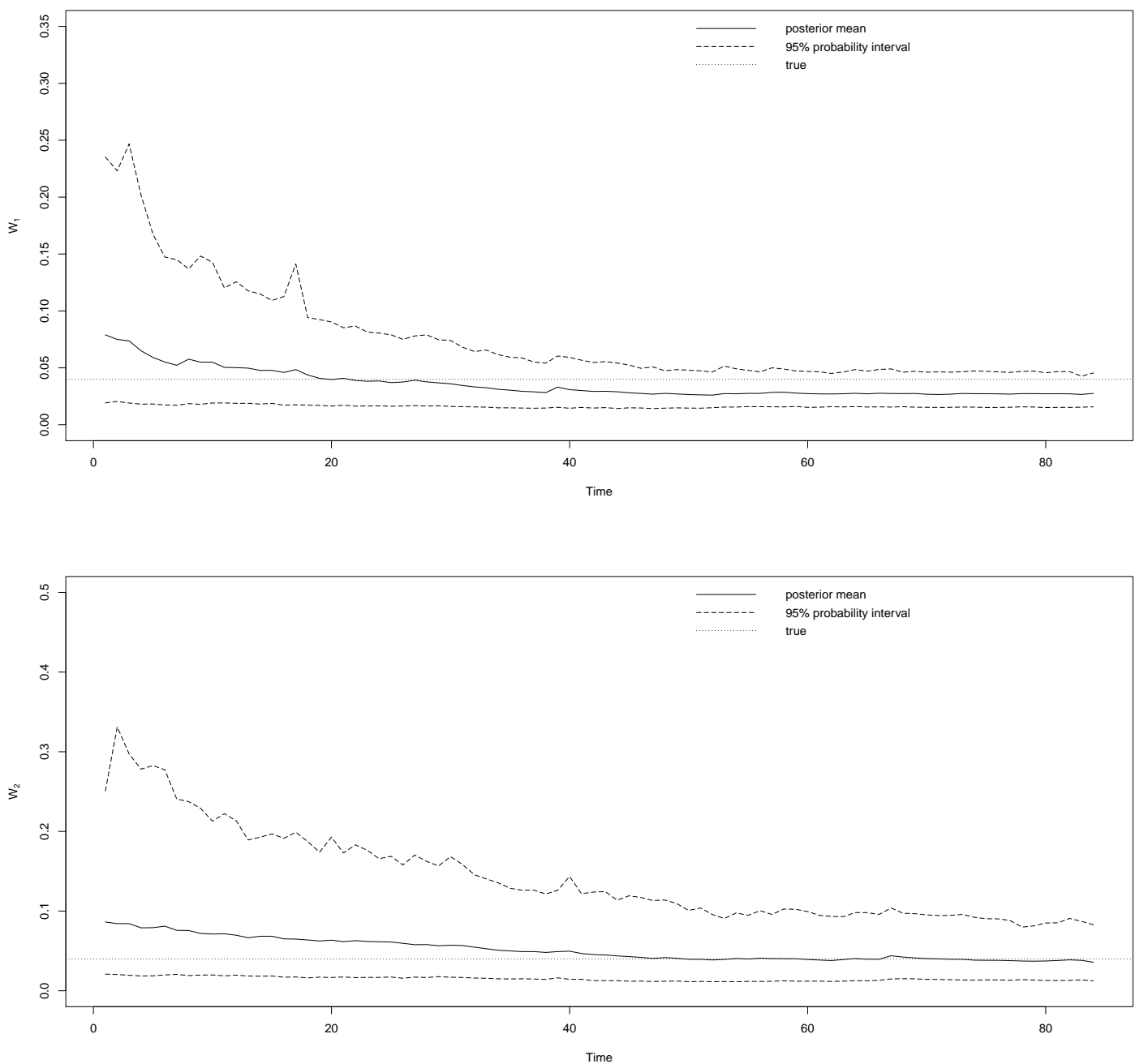

Figura 6.6: Gráfico da estimativa das componentes da variância $W$ do modelo BIZU via filtro PL. 

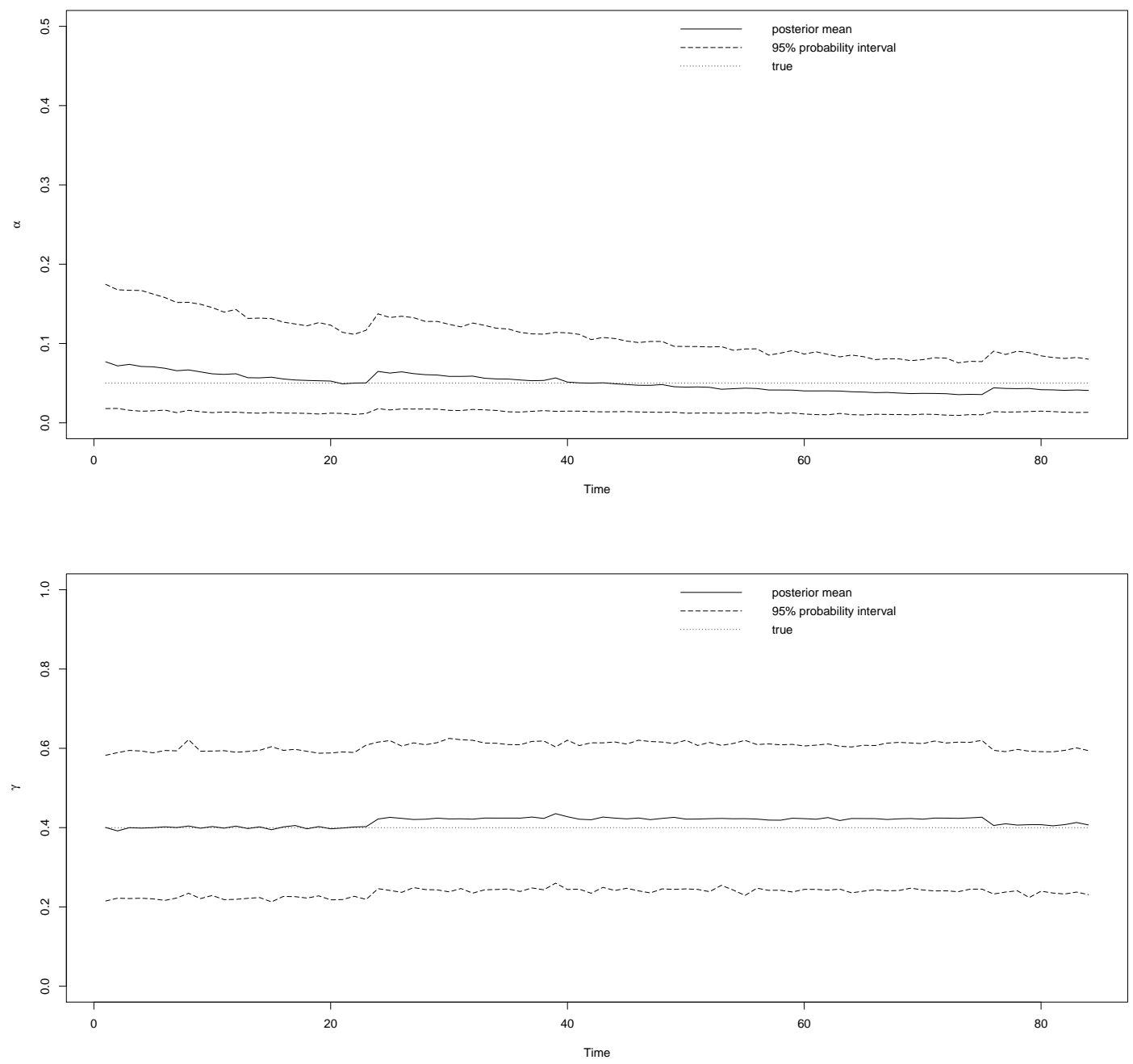

Figura 6.7: Gráfico da estimativa das probabilidades $\alpha$ e $\gamma$ do modelo BIZU via filtro PL. 
Para verificar se o processo de estimação se mantém adequado para série longa, o processo foi repetido para uma serie temporal de tamanho $T=840$, novamente. Conforme pode-se observar nas Figuras (6.10) e (6.11) em comparação com as Figuras (6.13) e (6.14), a superioridade do filtro PL fica ainda mais evidente na estimação dos parâmetros estáticos, principalmente no caso das probabilidades $\alpha$ e $\gamma$, onde a sequência de estimativas chegam perto de colapsar e se distanciam dos valores verdadeiros.

Foram fixados os valores de $\alpha=0,05$ e $\gamma=0,4$, representando o parâmetro de probabilidade da componente discreta do modelo. A variância do componente do erro da equação de evolução foi fixada em $W=\operatorname{diag}(0,04,0,0025)$. Os hiperparâmetros da priori inicial para os espaços de estados foram definidos por $m_{0}=(\operatorname{logit}(0,4), \log (400))^{\prime}$ e $C_{0}=\operatorname{diag}(0,25,0,0025), \theta_{0} \sim N\left(m_{0}, C_{0}\right)$.

Para ambos os filtros foram atribuídas as prioris: $\alpha_{0} \sim B(3,35)$ e $\gamma_{0} \sim B(10,15)$. Estes hiperparâmetros foram escolhidos afim de obter uma distribuição centrada na proporção de zeros e uns na série. As prioris para as componentes da variância do erro de evolução foram dadas por $W_{1}^{-1} \sim G(7 / 2,0,03 \times 7 / 2)$ e $W_{2}^{-1} \sim G(5 / 2,0,0015 \times 5 / 2)$, com $W_{0}=\operatorname{diag}\left(W_{1}, W_{2}\right)$ e os hiperparâmetros para a priori do espaço de estado inicial foram $m_{0}=\left(\operatorname{logit}\left(y_{1}\right),-2 \log \left(\operatorname{var}\left(y_{1: T}\right)\right)\right)^{\prime} \mathrm{e}$ $C_{0}=\operatorname{diag}\left(0,5^{2}, 0,05^{2}\right)$.

Em ambos os filtros há uma certa dificuldade em obter boa estimativa de $W=\operatorname{diag}\left(W_{1}, W_{2}\right)$, uma vez que sua distribuição a posteriori é bem sensível a escolha de sua priori. Portanto é importante usar procedimento para obter uma boa escolha de priori. Neste estudo foi usado um modelo simples dinâmico linear t-student com fator de desconto para obter uma estimativa inicial de $W$, e foi utilizadas prioris de distribuição gama centrada nos valores estimados via MLD.

No aspecto computacional o filtro LW foi mais eficiente por exigir um número menor de amostragem a cada iteração. Enquanto o filtro LW durou tempo aproximado de 35 minutos o filtro PL durou um pouco mais que 1 hora e 30 minutos de tempo computacional.

O filtro LW não apresentou resultados satisfatórios para estimar parte dos parâmetros fixos do modelo. Com a evidente vantagem do filtro PL sobre o filtro LW na qualidade da estimação dos modelos, o filtro PL será o processo de estimação utilizado nos próximos estudos apresentados.

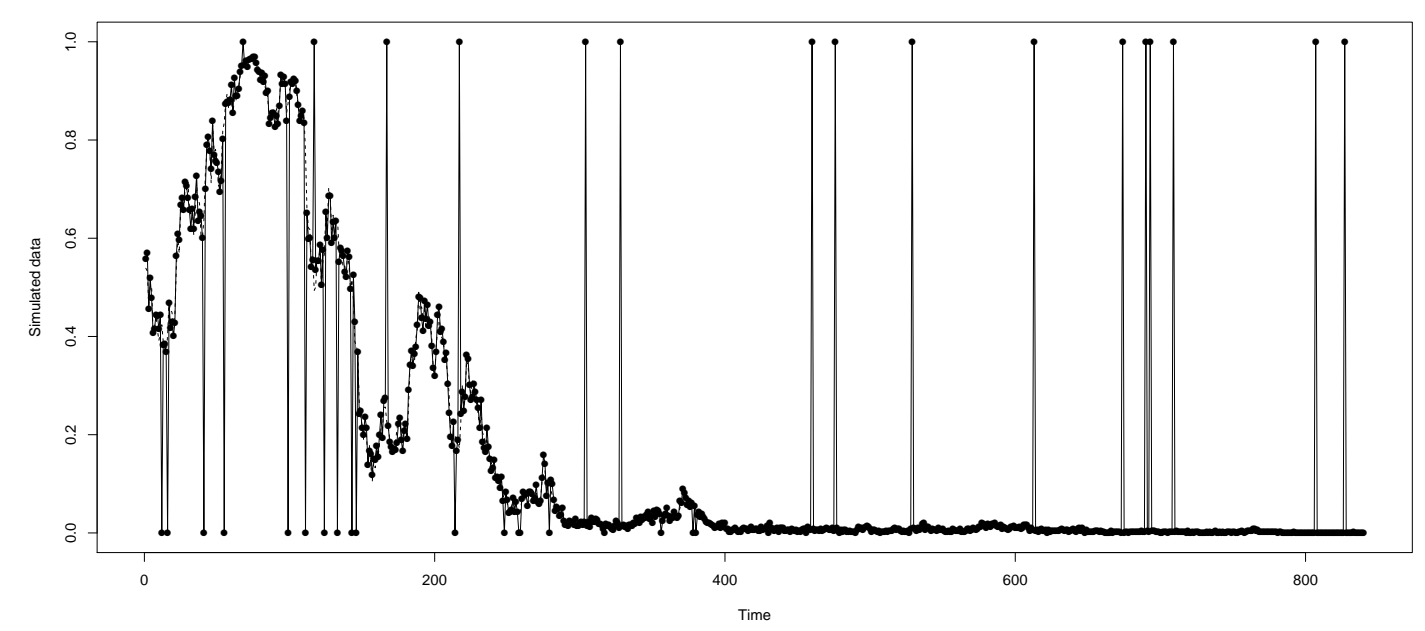

Figura 6.8: Gráficos dos dados simulados do modelo BIZU com passeio aleatório (linha contínua) e $\mu_{t}$ (linha pontilhada). 

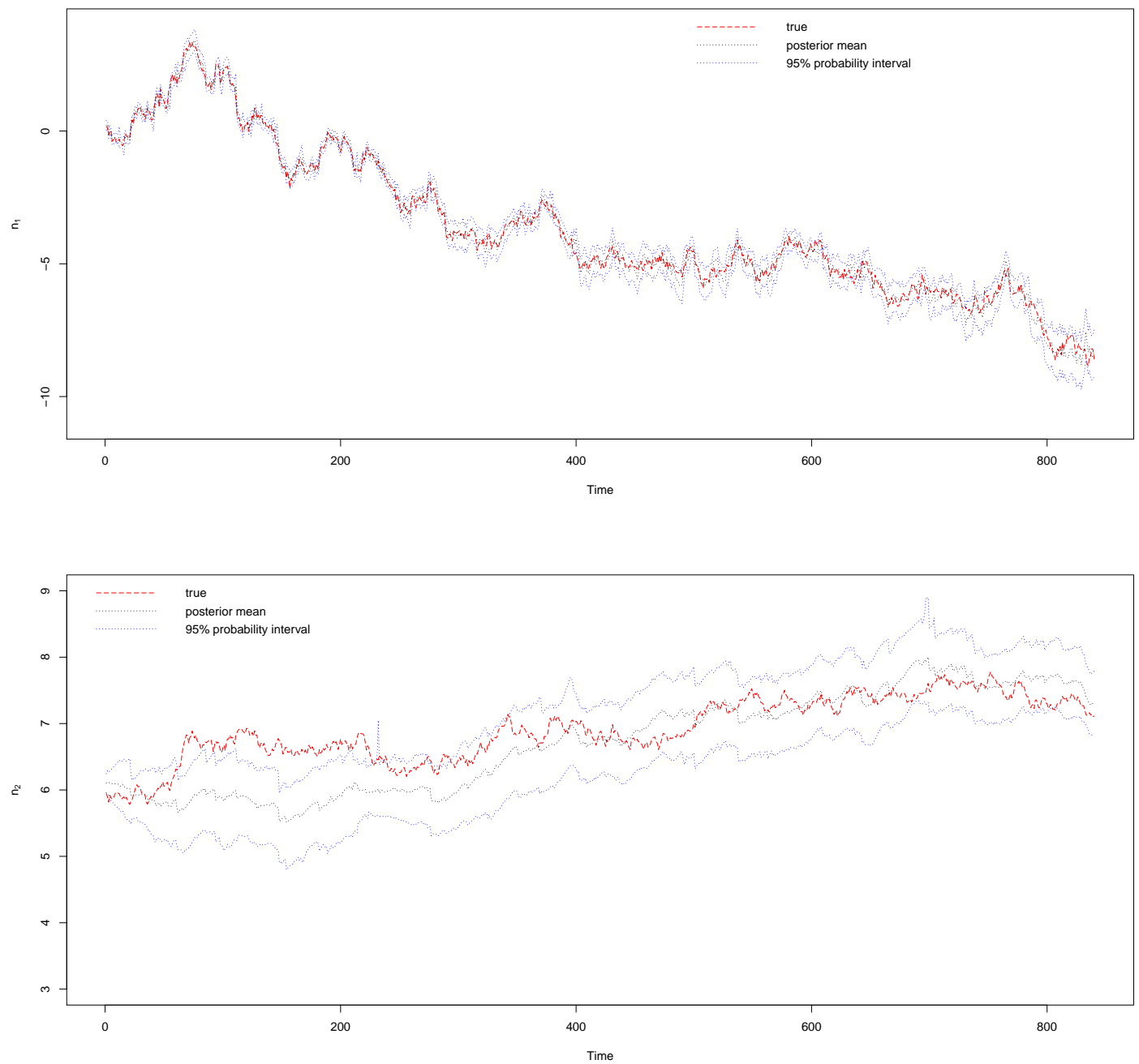

Figura 6.9: Gráficos dos preditores linearesde $\mu_{t}$ e $\phi_{t}$ do modelo BIZU estimados via filtro $L W$. 

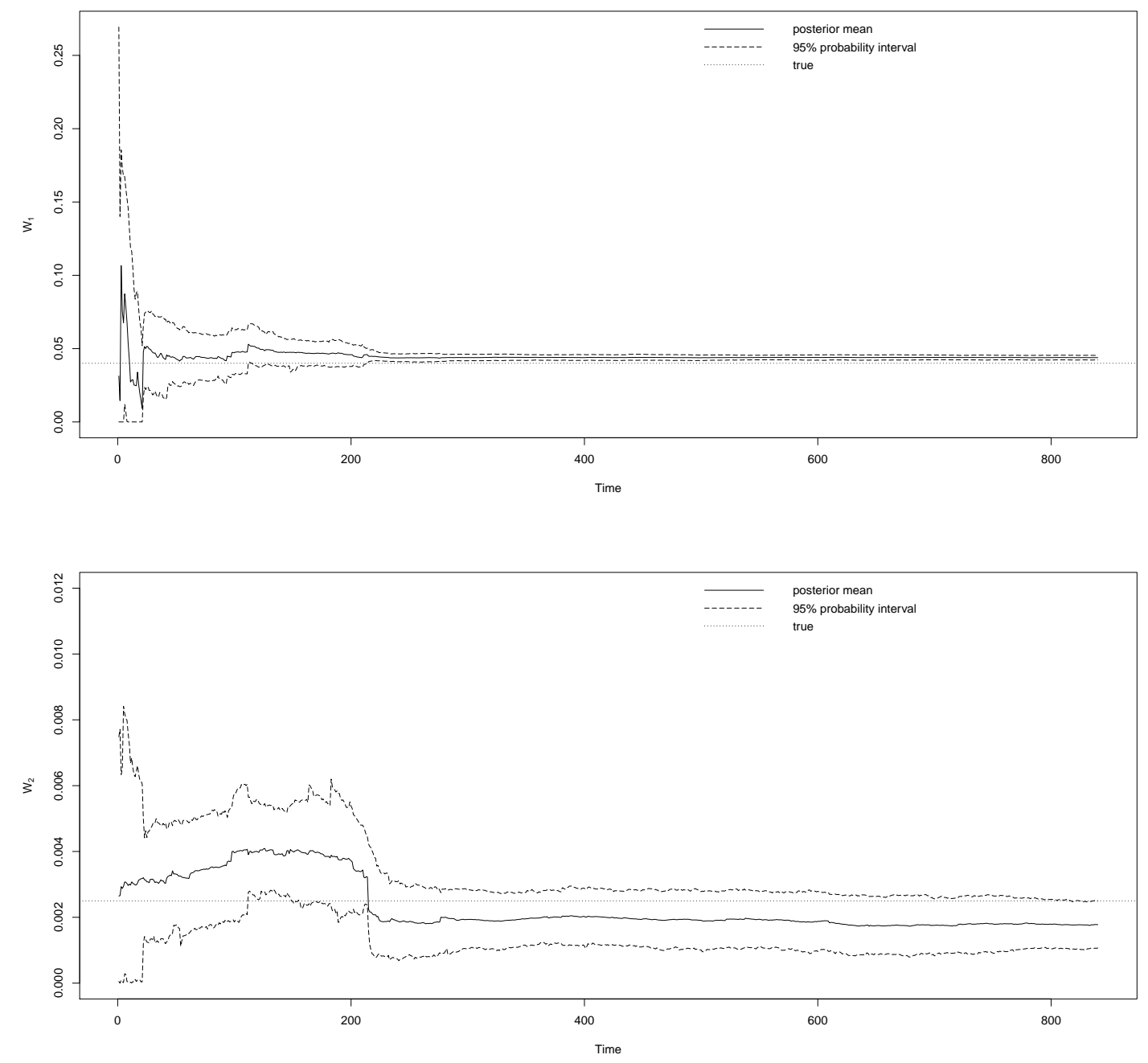

Figura 6.10: Gráfico da estimativa das componentes da variância $W$ do modelo BIZU via filtro $L W$. 

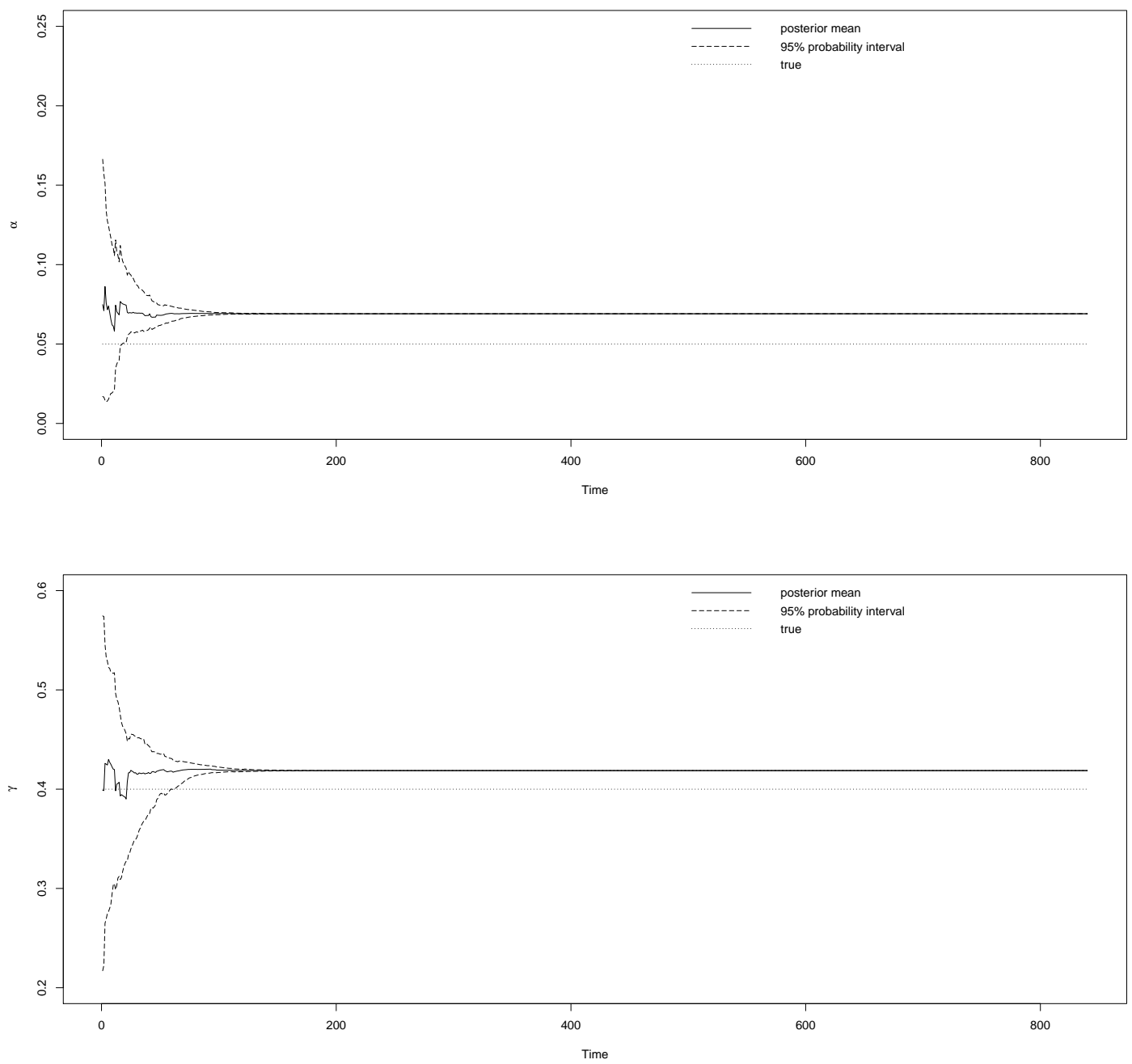

Figura 6.11: Gráfico da estimativa das probabilidades $\alpha$ e $\gamma$ do modelo BIZU via filtro $L W$. 

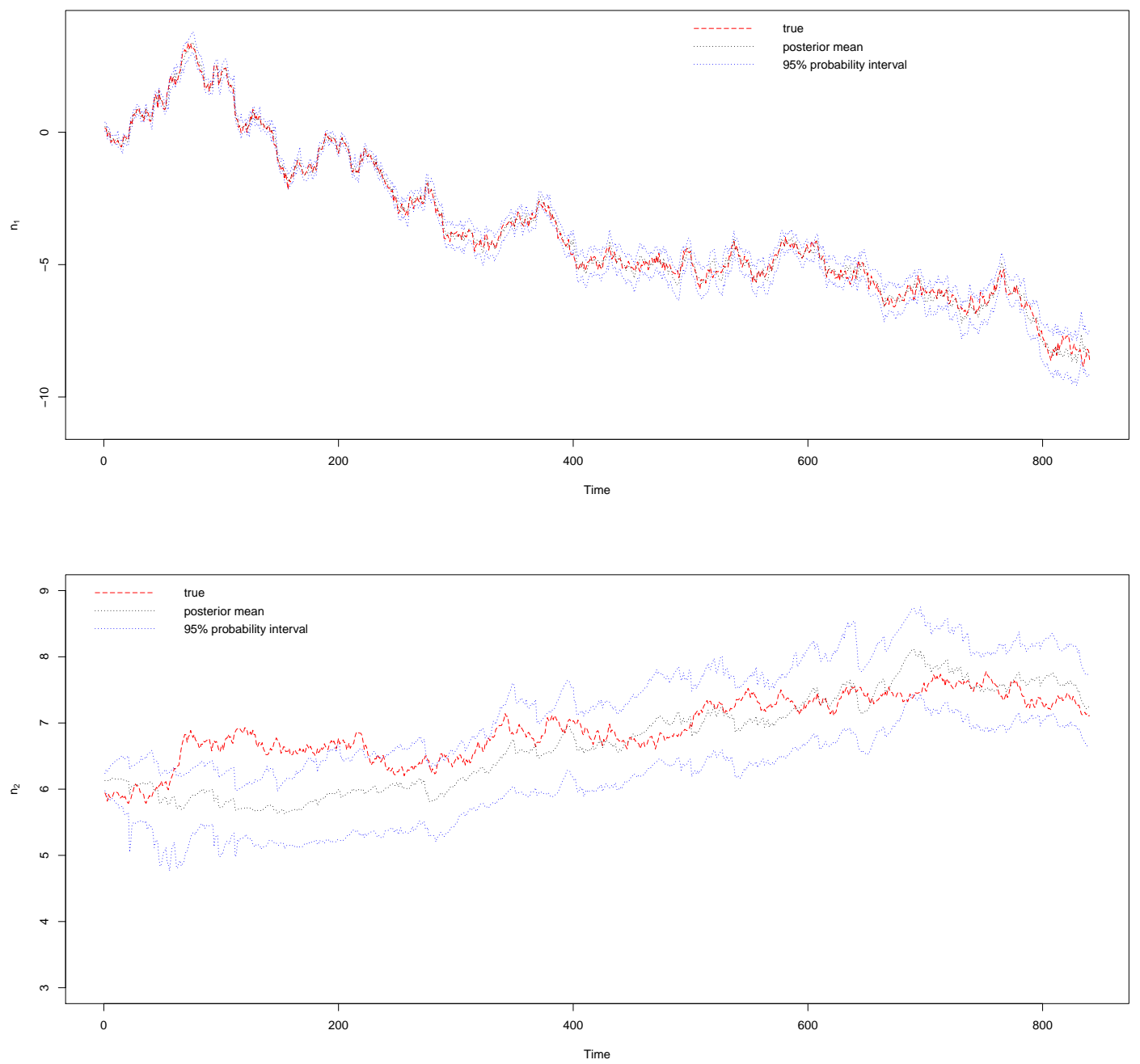

Figura 6.12: Gráficos dos preditores lineares de $\mu_{t}$ e $\phi_{t}$ do modelo BIZU estimados via filtro PL. 

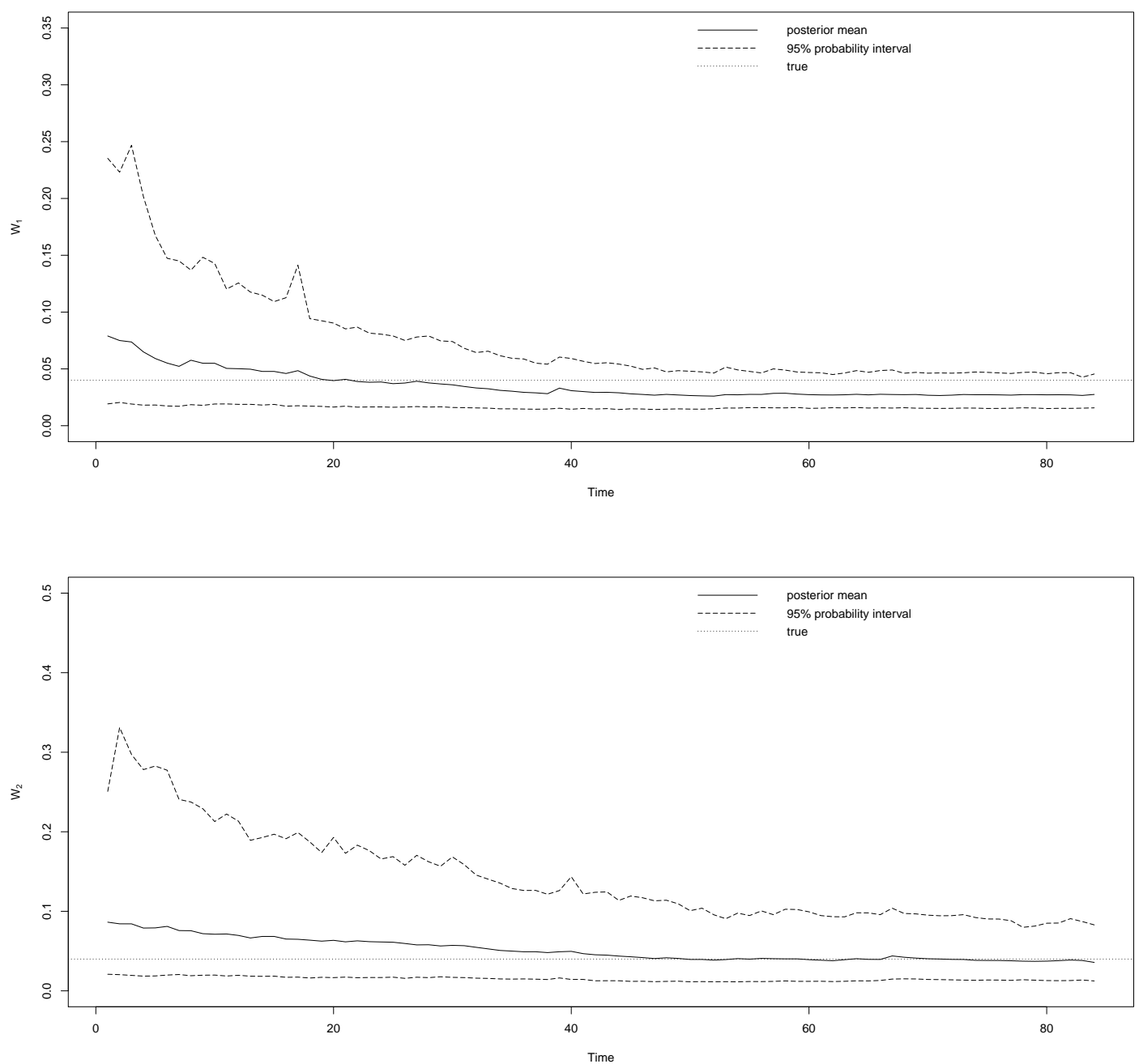

Figura 6.13: Gráfico da estimativa das componentes da variância $W$ do modelo BIZU via filtro PL. 

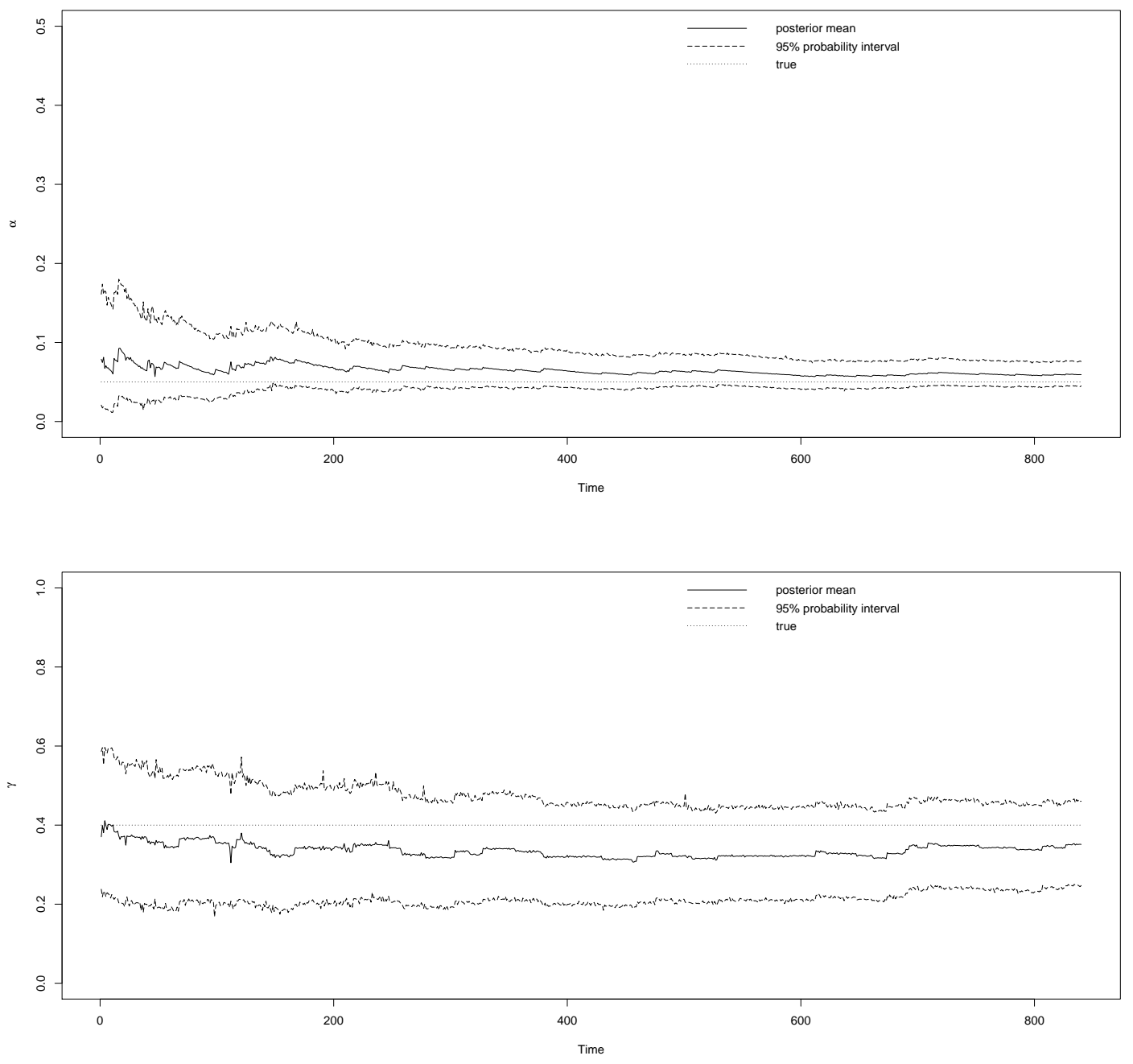

Figura 6.14: Gráfico da estimativa das probabilidades $\alpha$ e $\gamma$ do modelo BIZU via filtro PL. 


\subsubsection{Modelo com nível, tendência e sazonalidade}

Neste estudo, foi considerada a seguinte estrutura de modelo:

$$
\begin{aligned}
& \eta_{t}=F^{\prime} \theta_{t} \\
& \theta_{t}=G \theta_{t-1}+\omega, \quad \omega \sim N(0, W) .
\end{aligned}
$$

com $G$ composta por 3 matrizes bloco diagonais:

$$
J=\left(\begin{array}{ll}
1 & 1 \\
0 & 1
\end{array}\right), \quad P=\left(\begin{array}{cc}
-\mathbf{1}_{p-2}^{\prime} & -1 \\
\mathbf{I}_{p-2} & 1
\end{array}\right), \quad \text { e } \quad I_{1}=(1)
$$

onde $J$ representa a evolução de nível e tendência da média $\mu_{t}, P$ representa a evolução da sazonalidade de período de tamanho $p$ e $I_{1}$ corresponde a evolução de nível da precisão $\phi_{t}$. A matriz $F$ é dada por

$$
F=\left(\begin{array}{ccccc}
1 & 0 & 1 & \mathbf{0}_{p-2} & 0 \\
0 & 0 & 0 & \mathbf{0}_{p-2} & 1
\end{array}\right)
$$

Mais informações sobre a estrutura de modelos polinomiais podem ser obtidas em (West e Harrison, 1997, Cap.7).

Foi gerada uma série de tamanho $T=84$ com periodicidade $p=4$, e probabilidades $\alpha=0,05$ e $\gamma=0,4$. É importante ressaltar que assim como a matriz $G$, a matriz $W$ possui a mesma estrutura bloco-diagonal, o que permite que para o processo de estimação seja fragmentada em 3 sub-matrizes, permitindo a amostragem de variáveis com dimensões menores. A variância do componente do erro da equação de evolução foi fixada em $W_{1}=\operatorname{diag}(0,01,0,001), W_{2}=\operatorname{diag}\left(10^{-5}, 10^{-5}, 10^{-5}\right)$ e $W_{3}=0,001$, com $W=\operatorname{bdiag}\left(W_{1}, W_{2}, W_{3}\right)$, onde $\operatorname{bdiag}($.$) corresponde a construção bloco-diagonal$ das matrizes. Os hiperparâmetros da priori inicial para os espaços de estados foram definidos por $m_{0}=(0,35,0,005,0,18,0,005,0,005,5,3)^{\prime}$ e $C_{0}=\operatorname{bdiag}\left(C_{01}, C_{02}, C_{03}\right), C_{01}=\operatorname{diag}(0,001,0,0001)$, $C_{02}=\operatorname{diag}\left(10^{-5}, 10^{-5}, 10^{-5}\right)$ e $C_{03}=0,001, \theta_{0} \sim N\left(m_{0}, C_{0}\right)$.

Foram atribuídas as prioris: $\alpha_{0} \sim B(3,35)$ e $\gamma_{0} \sim B(10,15)$. Estes hiperparâmetros foram escolhidos afim de obter uma distribuição centrada na proporção de zeros e uns na série. As prioris para as componentes da variância do erro de evolução foram dadas por $W_{01}^{-1} \sim W(12 / 2, \operatorname{diag}(200,200))$, $W_{02}^{-1} \sim W\left(12 / 2, \operatorname{diag}\left(10^{3}, 10^{3}, 10^{3}\right)\right)$ e $W_{03}^{-1} \sim G(5 / 2,0,0005 \times 5 / 2), \operatorname{com} W_{0}=\operatorname{bdiag}\left(W_{01}, W_{02}, W_{03}\right)$ e os hiperparâmetros para a priori do espaço de estado inicial foram $m_{0}=\left(\operatorname{logit}\left(y_{1}\right) \times(0,85,0,005,0,18,0,005,0,005),-2 \log \left(\operatorname{var}\left(y_{1: T}\right)\right)\right)^{\prime}$ e $C_{0}=\operatorname{bdiag}\left(C_{01}, C_{02}, C_{03}\right)$, $C_{01}=\operatorname{diag}(0,01,0,001), C_{02}=\operatorname{diag}\left(10^{-4}, 10^{-4}, 10^{-4}\right)$ e $C_{03}=0,001$. O tempo computacional do processo foi de aproximadamente 21 minutos.

A Figura (6.16) a estimativa de $\eta_{1 t}$ e $\eta_{2 t}$, a Figura (6.17) as estimativas de $\mu_{t}$ e $\phi_{t}$. Na Figura (6.19) podemos observar as estimativas das principais componentes da matriz de variância $W$, assim como a Figura (6.20) apresenta a estimativa dos parâmetros de probabilidade $\alpha$ e $\gamma$. As Figuras (6.38) e (6.23) apresentam a distribuição preditiva $p\left(y_{t} \mid D_{t-1}\right)$ da componente contínua, sendo que na última foram desprezados os 10 últimos elementos do conjunto de dados parar calcular a previsão a $k$-passos a frente. Optamos por apresentar o gráfico da distribuição preditiva considerando somente a parte contínua do modelo $B I Z U$ para ter um melhor ilustração do qualidade do ajuste, dado que ao considerar a componente discreta os intervalos de credibilidade são deslocado para bem próximo das extremidades $\{0,1\}$. 


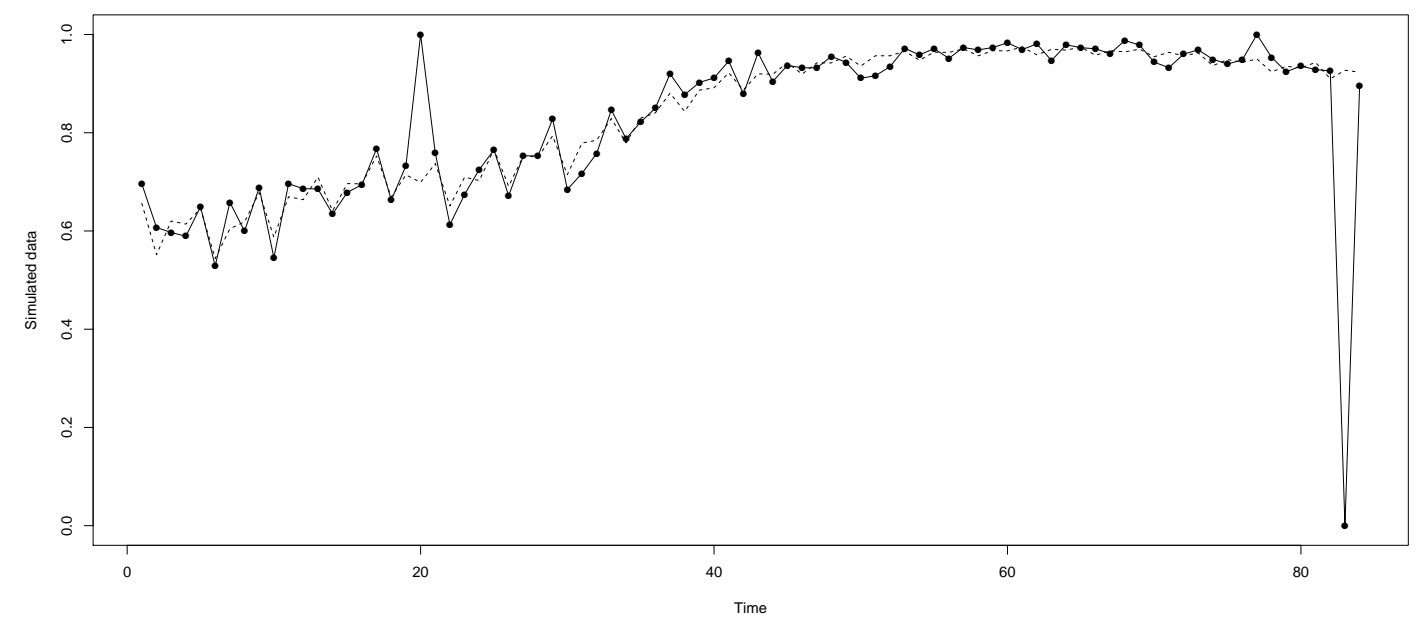

Figura 6.15: Gráfico da série simulada do modelo dinâmico BIZU com nivel, tendência e sazonalidade, com $y_{t}$ (linha continua) e $\mu_{t}$ (linha pontilhada).
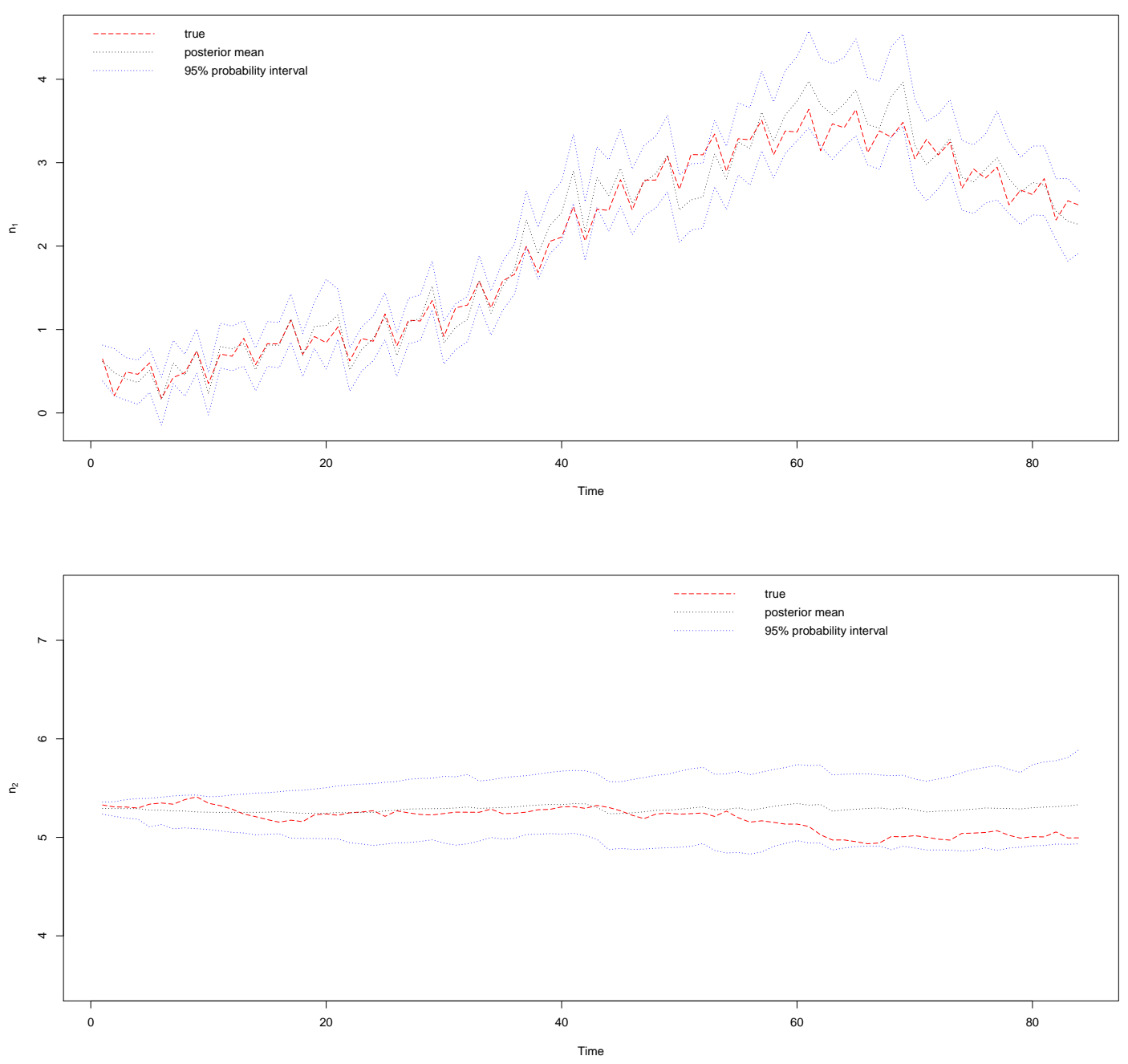

Figura 6.16: Gráfico dos preditores lineares de $\mu_{t}$ e $\phi_{t}$ do modelo dinâmico BIZU. 

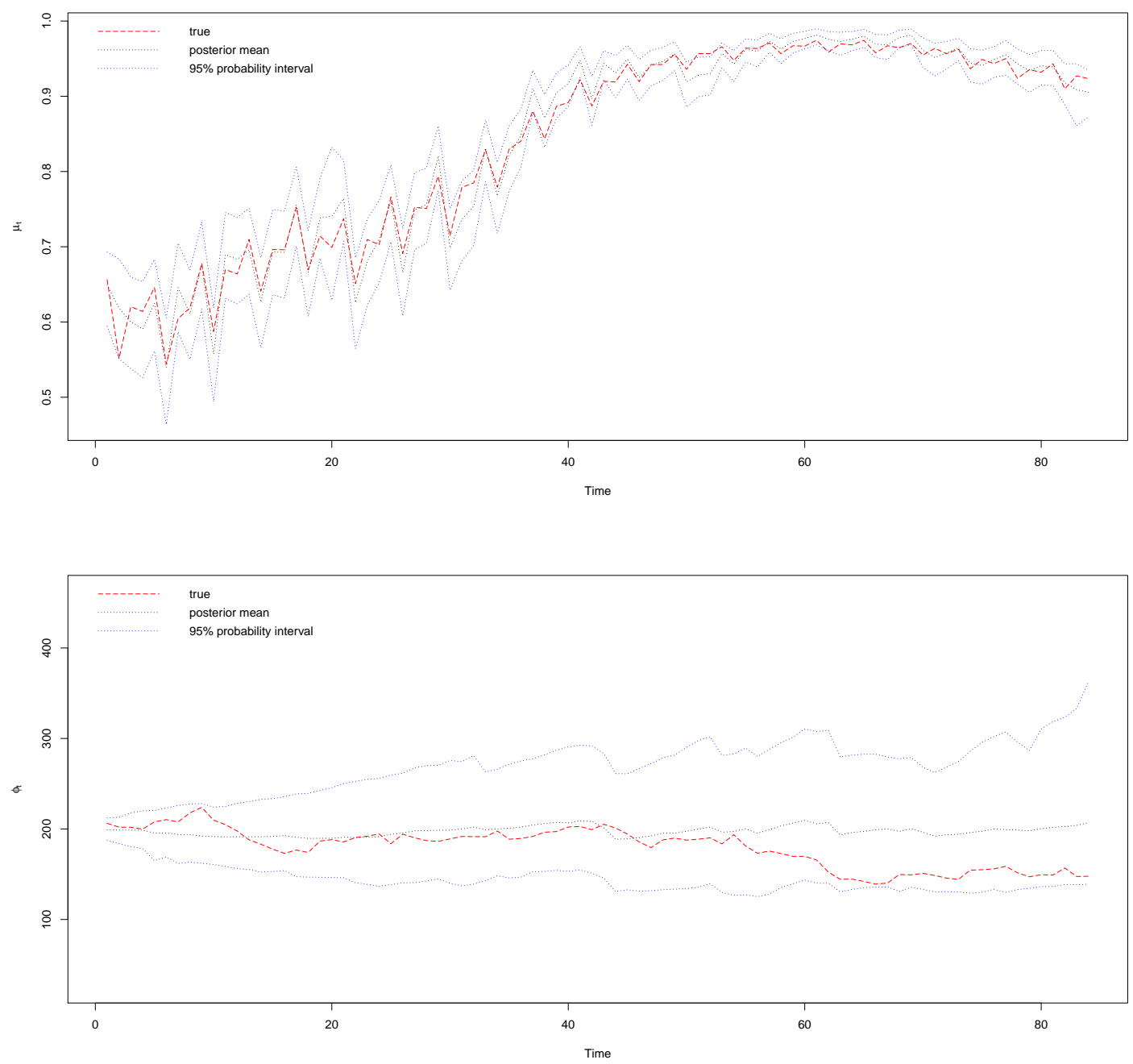

Figura 6.17: Gráfico dos estados $\mu_{t}$ e $\phi_{t}$ do modelo dinâmico BIZU. 
O parâmetro de precisão $\phi_{t}$ não é capaz de informar completamente o nível de dispersão da série, por isso a Figura (6.18) apresenta o desvio padrão estimado da componente contínua do modelo, dado por $\mu_{t}\left(1-\mu_{t}\right) /\left(1+\phi_{t}\right)$.

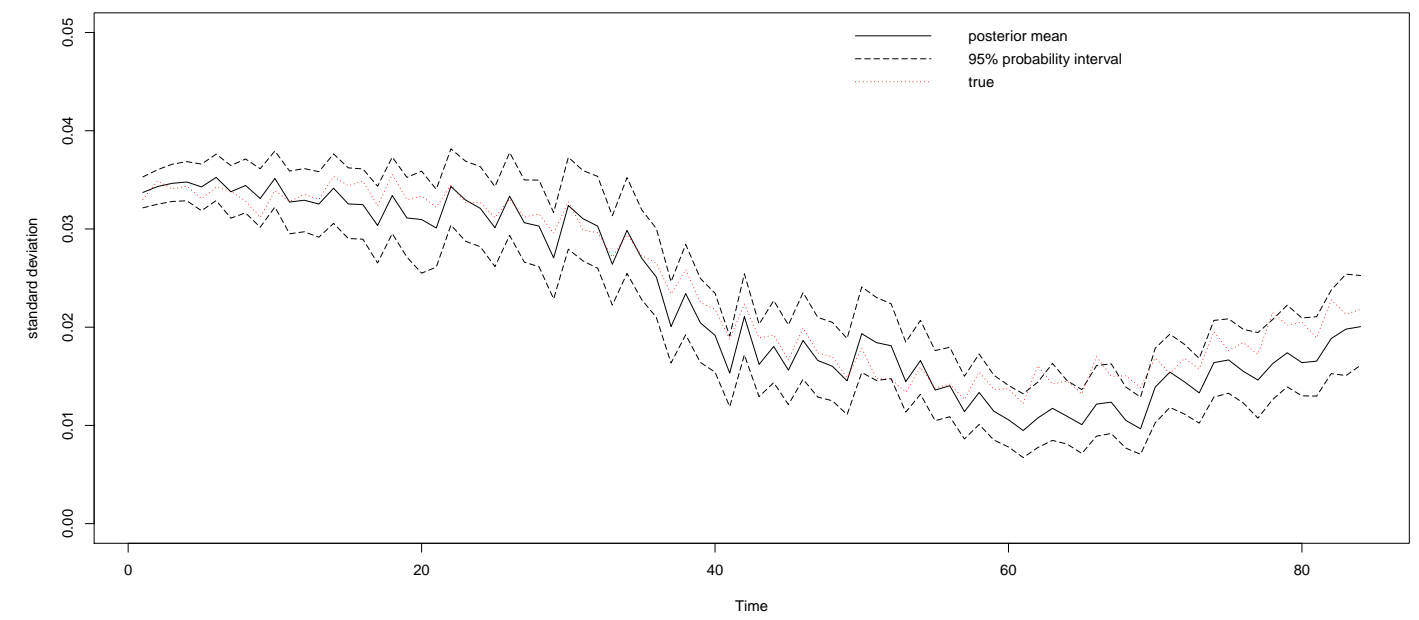

Figura 6.18: Gráfico do desvio padrão estimado da componente contínua do modelo dinâmico BIZU. 

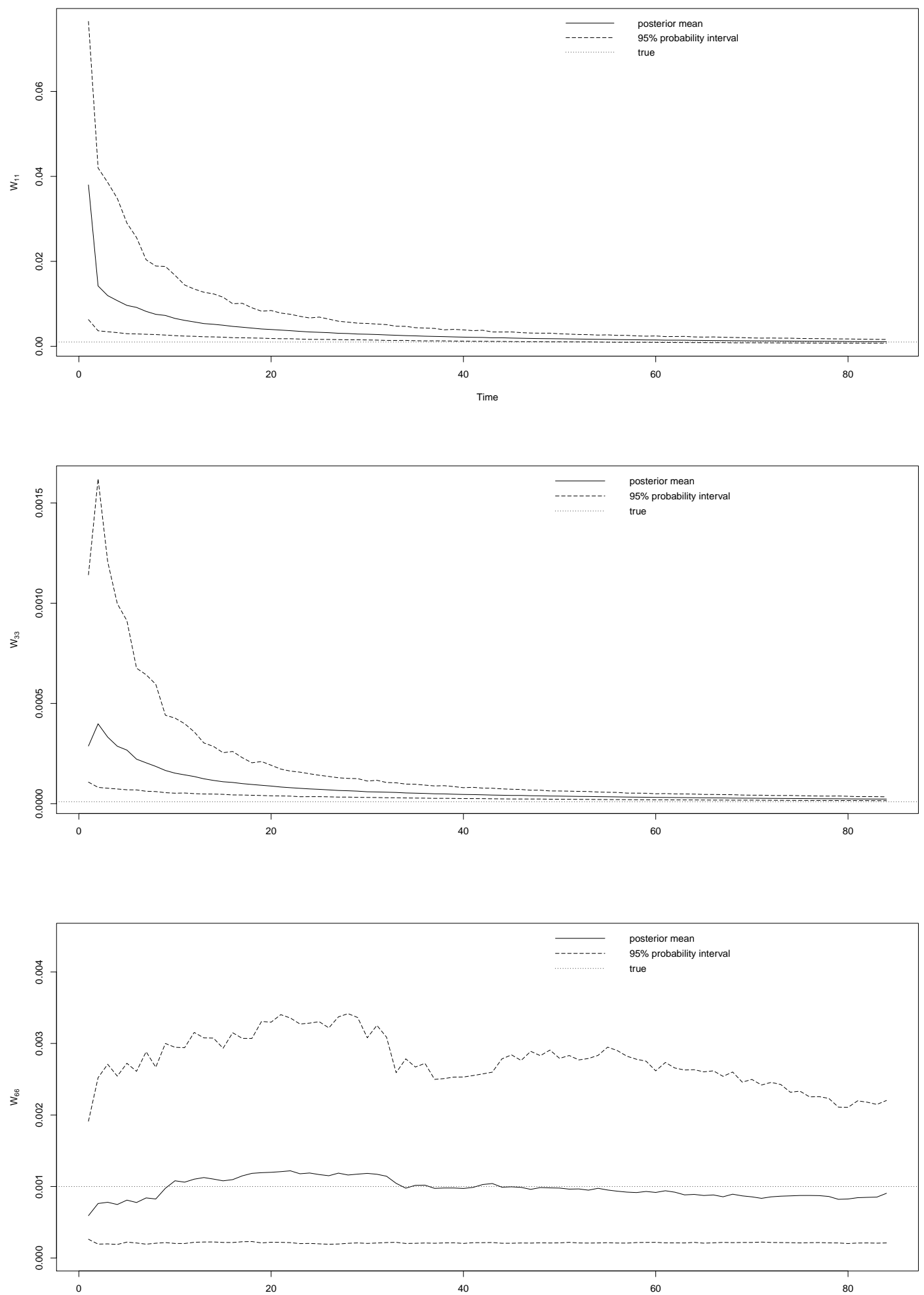

Figura 6.19: Gráfico das principais componentes da matriz de variância $W$ estimados no modelo BIZU. 

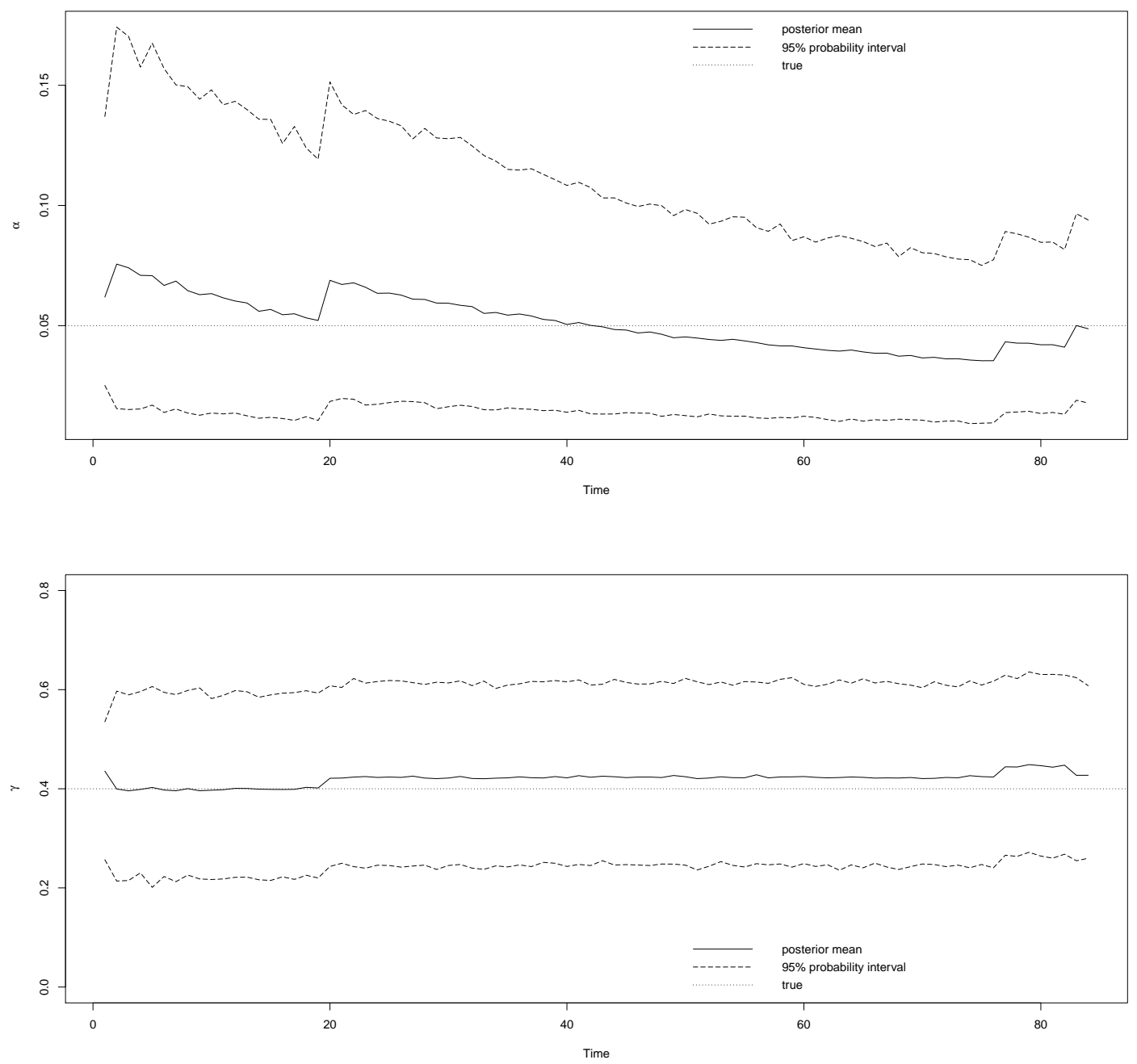

Figura 6.20: Gráfico da estimativa das probabilidades $\alpha$ e $\gamma$ do modelo BIZU. 


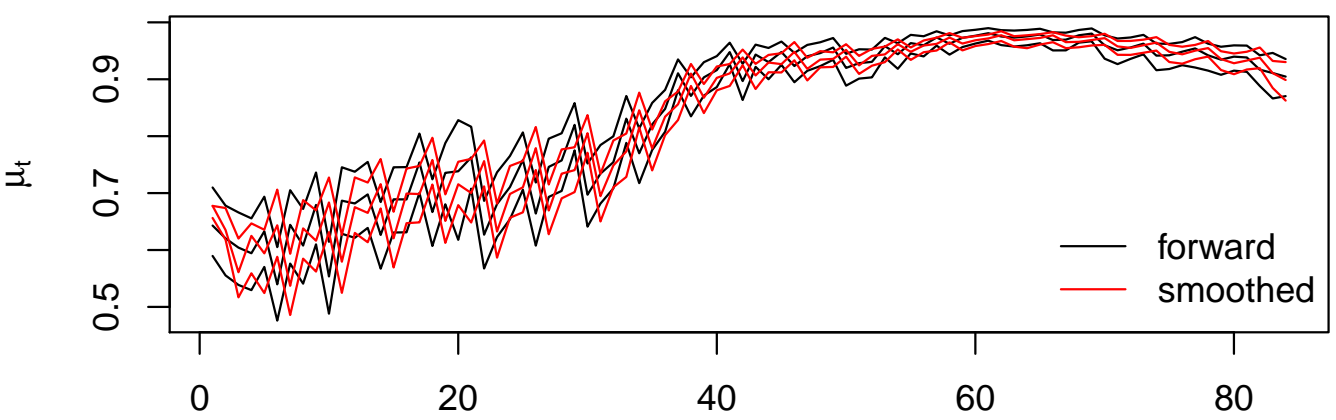

Time

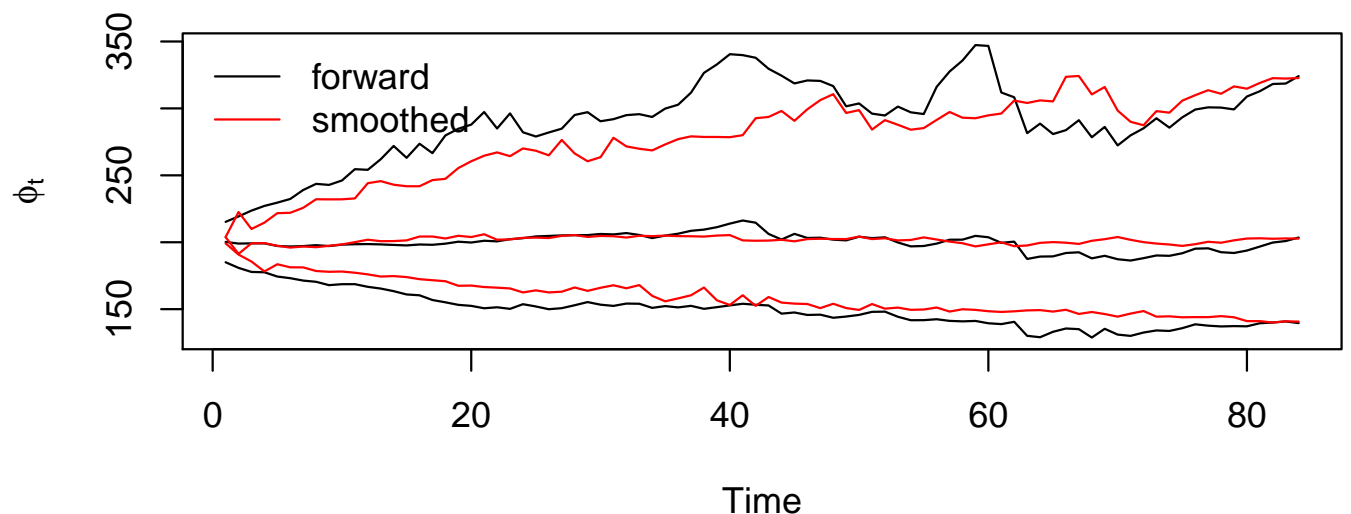

Figura 6.21: Gráfico dos preditores lineares $\eta_{t}$ suavizados em comparação aos estimados via o processo de filtragem.

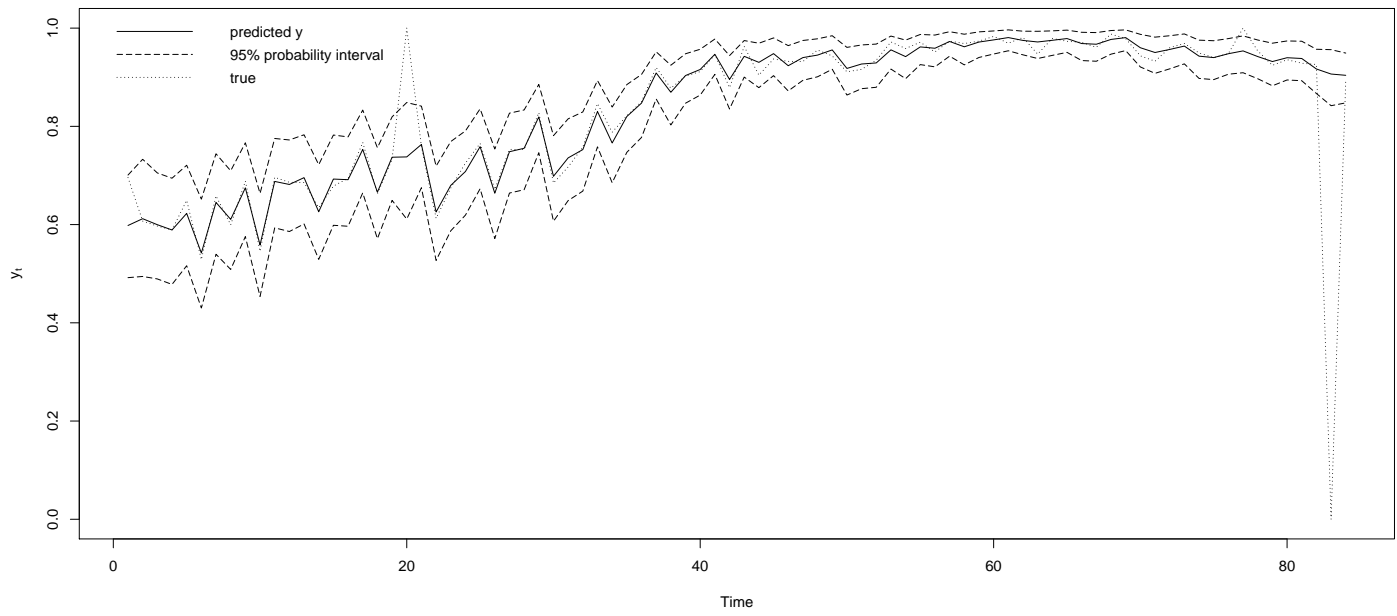

Figura 6.22: Gráfico da distribuição preditiva $p\left(y_{t} \mid D_{t-1}\right)$ da componente contínua do modelo dinâmico $B I Z U$. 


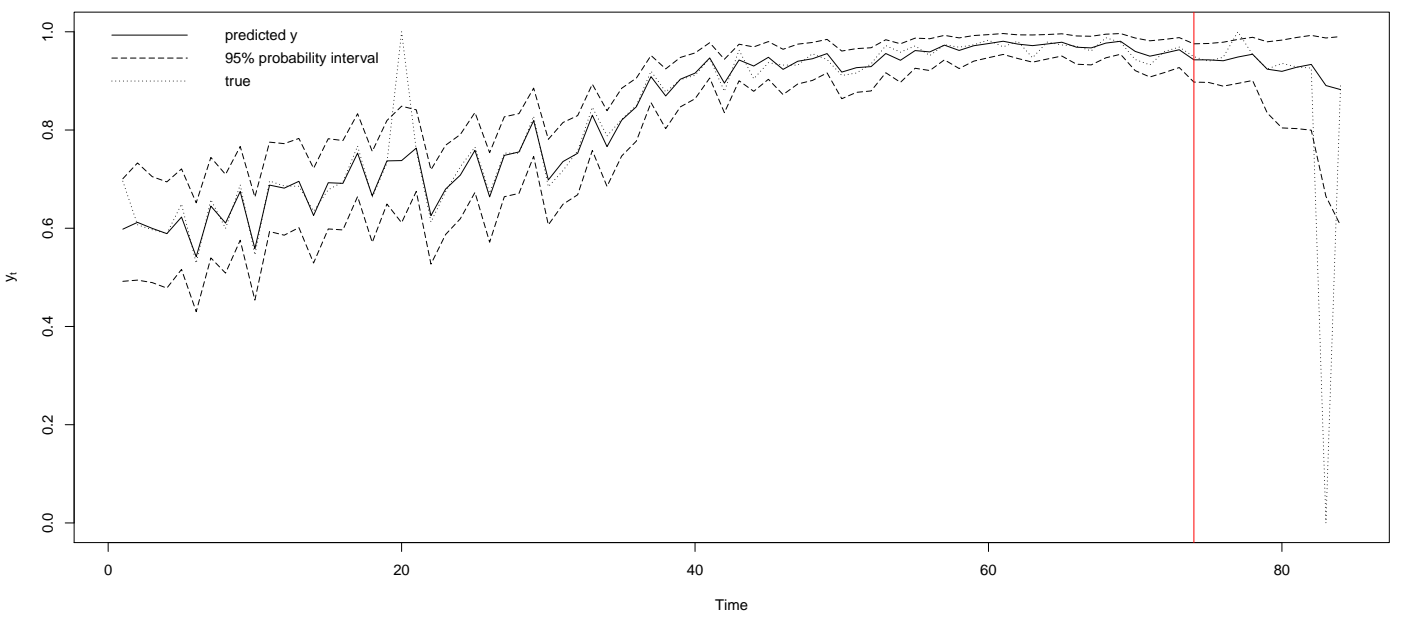

Figura 6.23: Gráfico da distribuição preditiva $p\left(y_{t} \mid D_{t-1}\right)$ da componente contínua do modelo dinâmico $B I Z U$ com previsão a 10 passos a frente. 


\subsubsection{Modelo com covariáveis}

Neste estudo, foi considerada a seguinte estrutura de modelo:

$$
\begin{aligned}
\eta_{t} & =F^{\prime} \theta_{t} \\
\theta_{t} & =G \theta_{t-1}+\beta_{1} x_{1 t}+\omega, \quad \omega \sim N(0, W) \\
\eta_{t}^{*} & =\log (\gamma /(1-\gamma))=\beta_{2} x_{2 t}+\nu, \quad \nu \sim N(0, V) .
\end{aligned}
$$

com $G$ e $F$ descritas como em (6.1.2). Neste caso, temos que considerar mais algumas estatísticas suficientes para o filtro PL, uma vez que o conjunto de parâmetros estático é dado por $\left(\beta_{1}, \beta_{2}, \alpha, V, W\right)$. Considerando as prioris:

$$
\begin{aligned}
\beta_{1} & \sim N\left(b_{01}, B_{01}\right), \\
\beta_{2} & \sim N\left(b_{02}, B_{02}\right), \\
V^{-1} & \sim G\left(v_{0}, u_{0}\right) .
\end{aligned}
$$

Temos as posterioris condicionais completas até o tempo $t$ dadas por:

$$
\begin{aligned}
\left(\beta_{1} \mid \theta_{1: t}, y_{1: t}, \beta_{2}, \gamma, V, W\right) & \sim N\left(\left(\sum_{i=1}^{t} x_{1 i}\left(\theta_{i}-G \theta_{i-1}\right) W^{-1}+\beta_{01}\left(B_{01}\right)^{-1}\right) * B_{1}^{-1}, B_{1}\right), \\
\left(\beta_{2} \mid \theta_{1: t}, y_{1: t}, \beta_{1}, \gamma, V, W\right) & \sim N\left(\left(\frac{\sum_{i=1}^{t} x_{2 i} \eta_{i}^{*}}{V}+\frac{\beta_{02}}{B_{02}}\right) * B_{2}^{-1}, B_{2}\right), \\
\left(V^{-1} \mid \theta_{1: t}, y_{1: t}, \beta_{1}, \beta_{2}, \gamma, W\right) & \sim G\left(v_{0}+t / 2, u_{0}+\sum_{i=1}^{t}\left(\eta_{i}^{*}-\beta_{2} x_{2 i}\right)^{2}\right) .
\end{aligned}
$$

com $B_{1}=\left(\sum_{i=1}^{t} x_{1 i}^{2}\right) W^{-1}+B_{01}^{-1}$ e $B_{2}=\left(\sum_{i=1}^{t} x_{2 i}^{2}\right) / V+1 / B_{02}$. Neste caso, temos o conjunto de estatísticas suficientes dado por

$$
\left(\sum_{i=1}^{t} \mathbb{1}_{\{0,1\}}\left(y_{i}\right), \sum_{i=1}^{t} x_{1 i}^{2}, \sum_{i=1}^{t} x_{1 i}\left(\theta_{i}-G \theta_{i-1}\right), \sum_{i=1}^{t} x_{2 i}^{2}, \sum_{i=1}^{t} x_{2 i} \eta_{i}^{*}, \sum_{i=1}^{t}\left(\eta_{i}^{*}-\beta_{2} x_{2 i}\right)^{2}, S S_{\theta_{1: t}}\right) .
$$

Foi gerada uma série de tamanho $T=84$ com periodicidade $p=4$, probabilidade $\alpha=0,2$, as covariáveis foram simuladas a partir de distribuições uniformes e os parâmetros das covariáveis foram fixados em $\beta_{1}=0,002, \beta_{2}=0,4$ e $V=0,001$. A variância do componente do erro da equação de evolução foi fixada em $W_{1}=\operatorname{diag}(0,001,0,0001), W_{2}=\operatorname{diag}\left(10^{-5}, 10^{-5}, 10^{-5}\right)$ e $W_{3}=0,001$, com $W=\operatorname{bdiag}\left(W_{1}, W_{2}, W_{3}\right)$. Os hiperparâmetros da priori inicial para os espaços de estados foram definidos por $m_{0}=(-0,85,0,005,0,18,0,005,0,005,5,3)^{\prime}$ e $C_{0}=\operatorname{bdiag}\left(C_{01}, C_{02}, C_{03}\right)$, $C_{01}=\operatorname{diag}(0,01,0,001), C_{02}=\operatorname{diag}\left(10^{-4}, 10^{-4}, 10^{-4}\right)$ e $C_{03}=0,001, \theta_{0} \sim N\left(m_{0}, C_{0}\right)$.

Foram atribuídas as prioris: $\alpha_{0} \sim B(3,35)$ e $\gamma_{0} \sim B(10,15)$. Estes hiperparâmetros foram escolhidos afim de obter uma distribuição centrada na proporção de zeros e uns na série. As prioris dos parâmetros relacionados as covariáveis foram dados por $\beta_{01} \sim N(0,0,01), \beta_{02} \sim N(0,4,0,08)$ e $V_{0}^{-1} \sim G(4,0,005)$. As prioris para as componentes da variância do erro de evolução foram dadas por $W_{01}^{-1} \sim W(12 / 2, \operatorname{diag}(200,200)), W_{02}^{-1} \sim W\left(12 / 2, \operatorname{diag}\left(10^{3}, 10^{3}, 10^{3}\right)\right)$ e $W_{03}^{-1} \sim G(5 / 2,0,005 \times$ $5 / 2)$, com $W_{0}=\operatorname{bdiag}\left(W_{01}, W_{02}, W_{03}\right)$ e os hiperparâmetros para a priori do espaço de estado inicial foram $m_{0}=\left(\operatorname{logit}\left(y_{1}\right) \times(0,85,0,005,0,18,0,005,0,005),-2 \log \left(\operatorname{var}\left(y_{1: T}\right)\right)\right)^{\prime}$ e $C_{0}=$ $\operatorname{bdiag}\left(C_{01}, C_{02}, C_{03}\right), C_{01}=\operatorname{diag}(0,05,0,01), C_{02}=\operatorname{diag}\left(10^{-3}, 10^{-3}, 10^{-3}\right)$ e $C_{03}=0,001 . \mathrm{O}$ tempo computacional do processo foi de aproximadamente 28 minutos.

A Figura (6.25) a estimativa de $\eta_{1 t}$ e $\eta_{2 t}$, a Figura (6.26) as estimativas de $\mu_{t}$ e $\phi_{t}$. Na Figura (6.28) podemos observar as estimativas das principais componentes da matriz de variância $W$, na Figura (6.29) apresenta a estimativa dos parâmetros $\beta_{1}, \beta_{2}$ e $V$. A Figura (6.30) apresenta a estimativa da probabilidade $\gamma$. A Figura (6.32) apresenta a distribuição preditiva $p\left(y_{t} \mid D_{t-1}\right)$ da 
componente contínua do modelo, sendo que na última foram desprezados os 10 últimos elementos do conjunto de dados parar calcular a previsão a $k$-passos a frente.

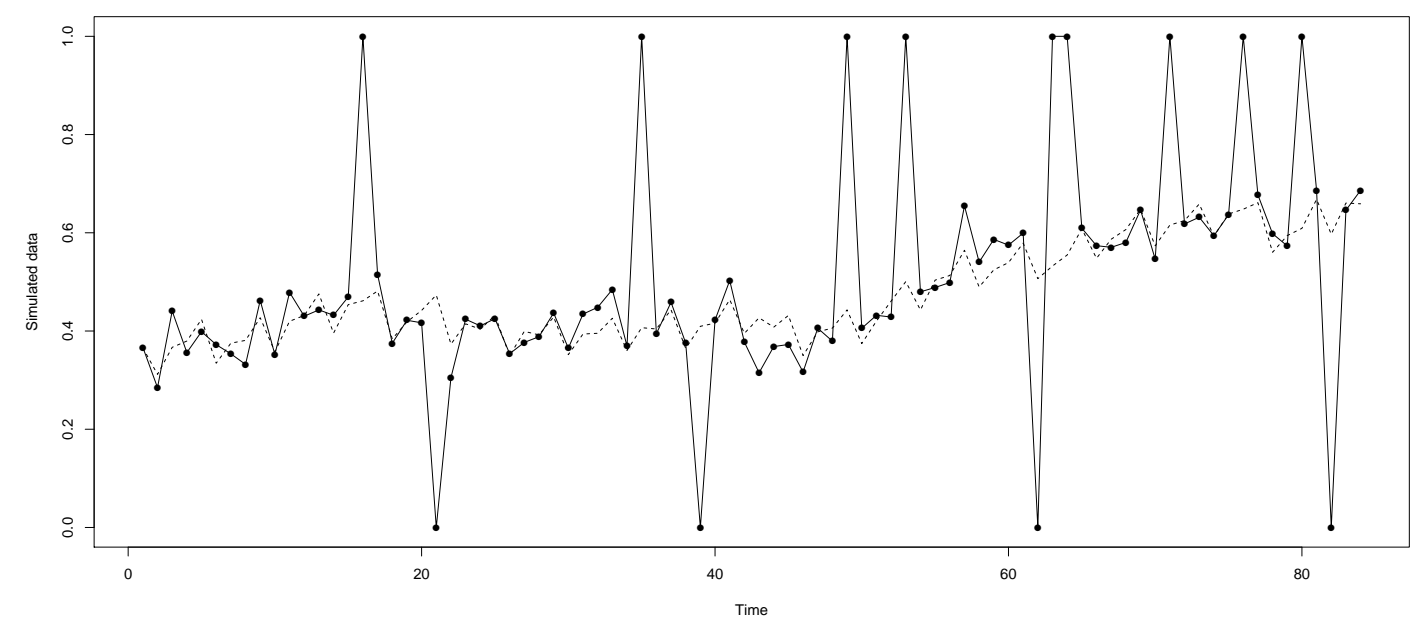

Figura 6.24: Gráfico da série simulada do modelo dinâmico BIZU com nível, tendência e sazonalidade e covariáveis, com $y_{t}$ (linha contínua) e $\mu_{t}$ (linha pontilhada). 

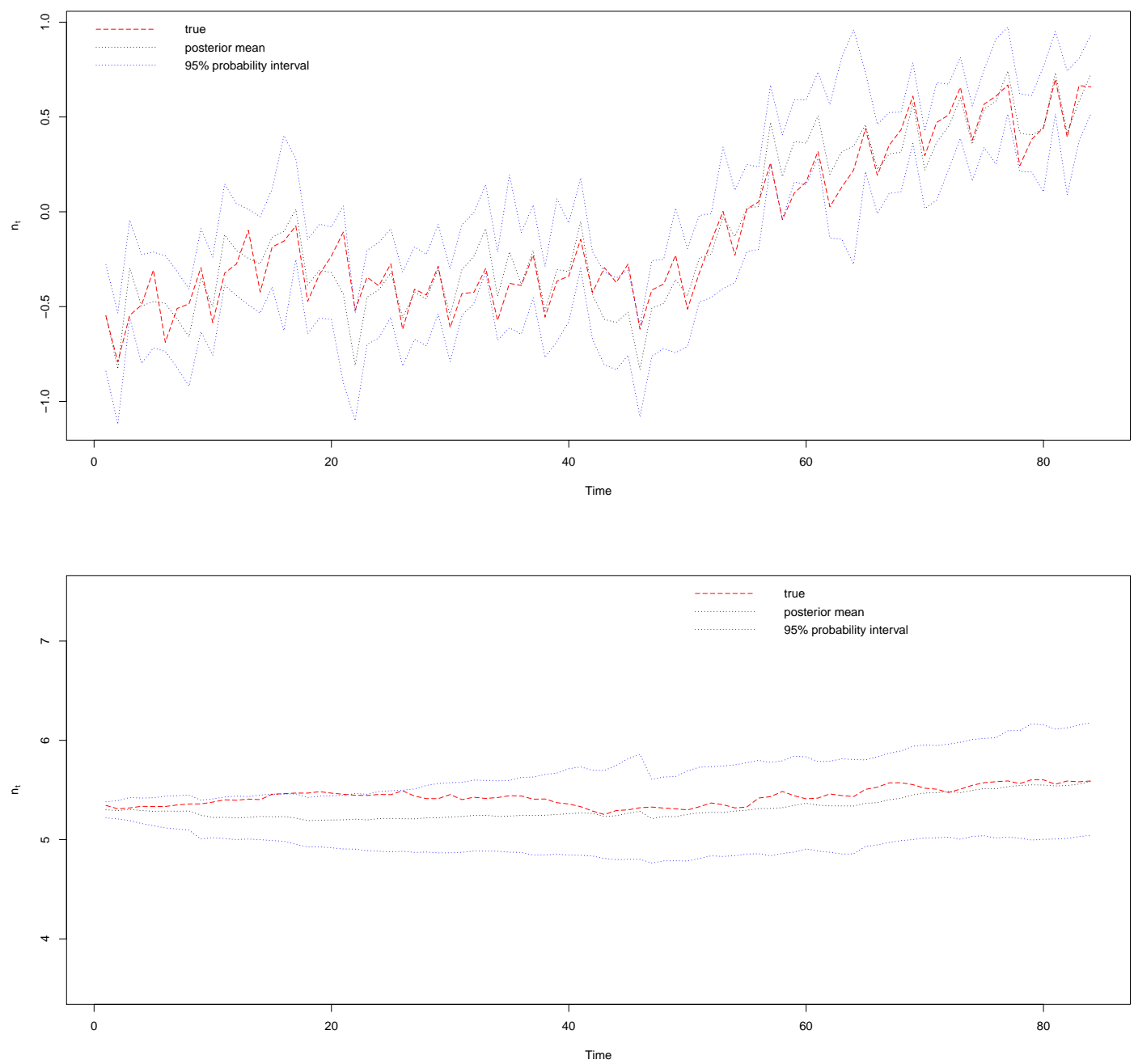

Figura 6.25: Gráfico dos preditores lineares de $\mu_{t}$ e $\phi_{t}$ do modelo dinâmico BIZU. 

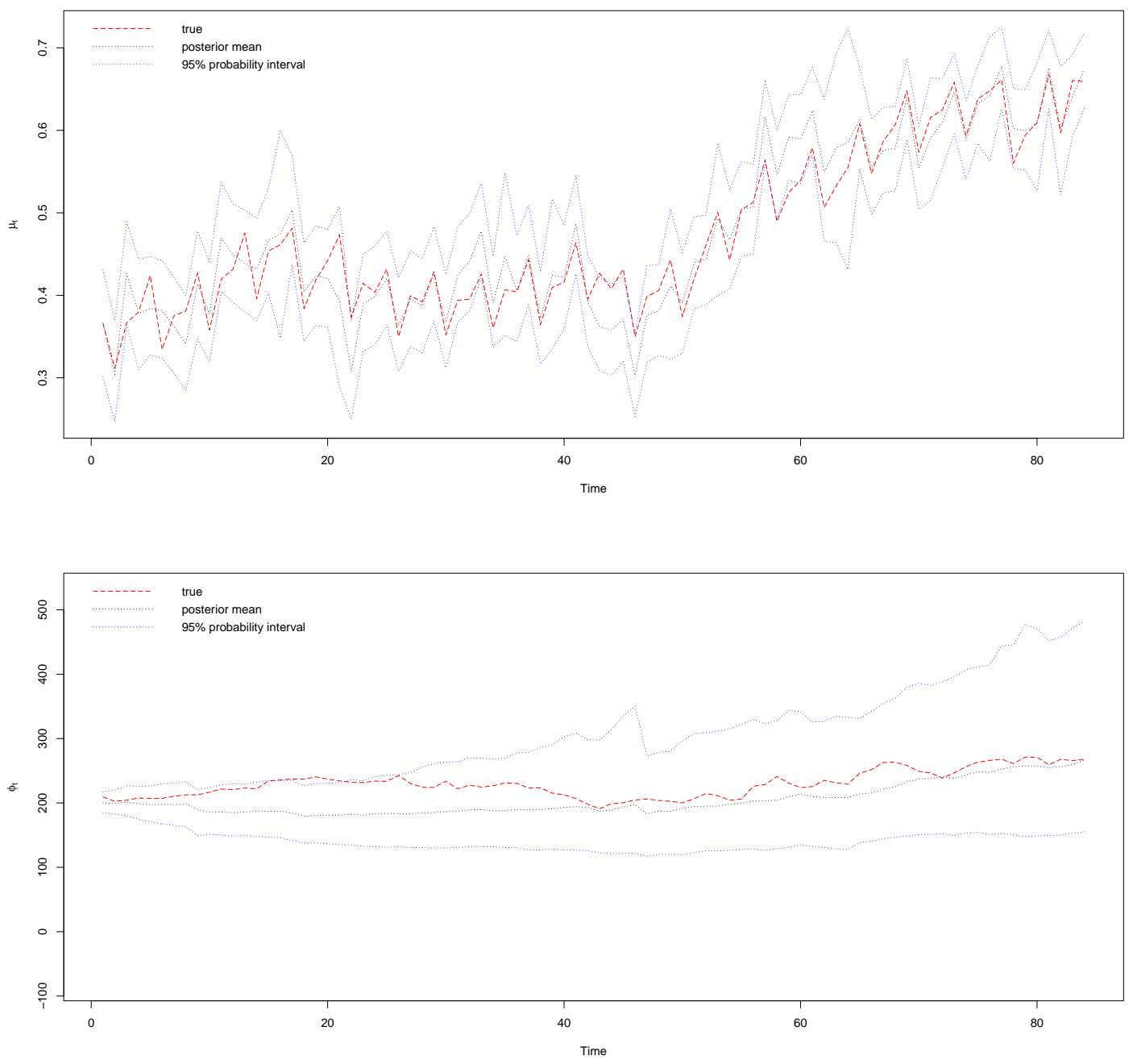

Figura 6.26: Gráfico dos estados $\mu_{t}$ e $\phi_{t}$ do modelo dinâmico BIZU.

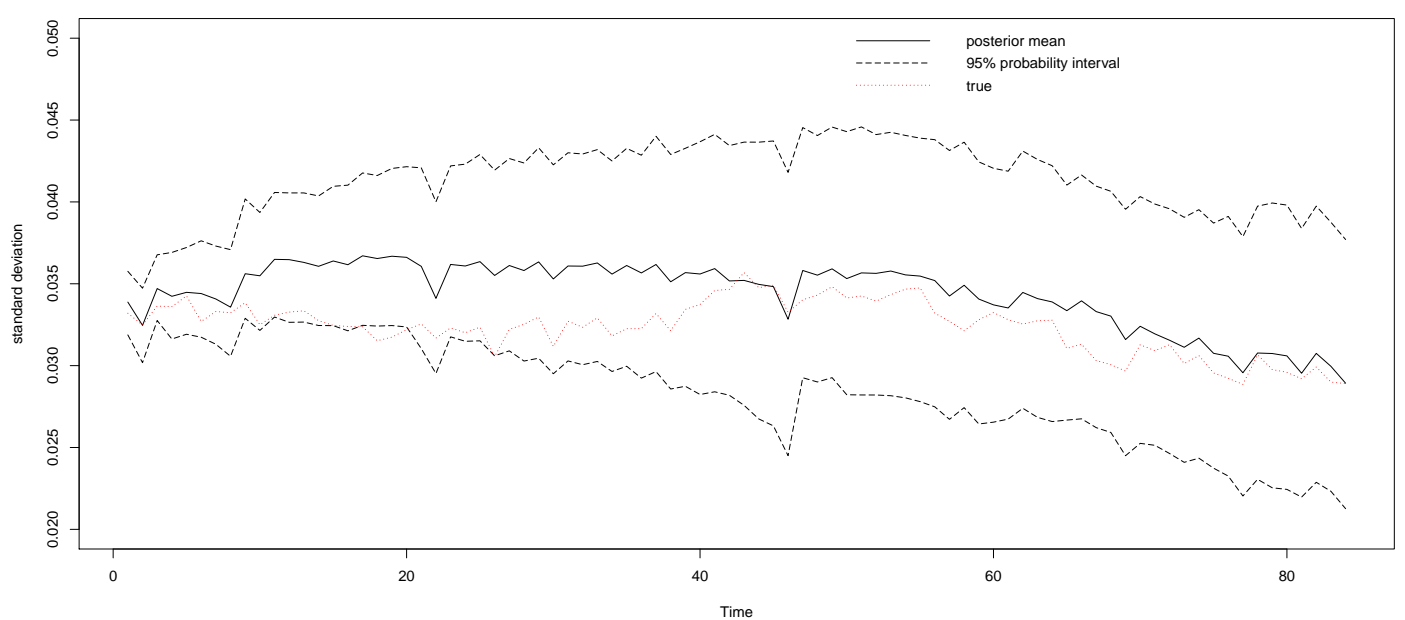

Figura 6.27: Gráfico do desvio padrão estimado da componente contínua do modelo dinâmico BIZU. 

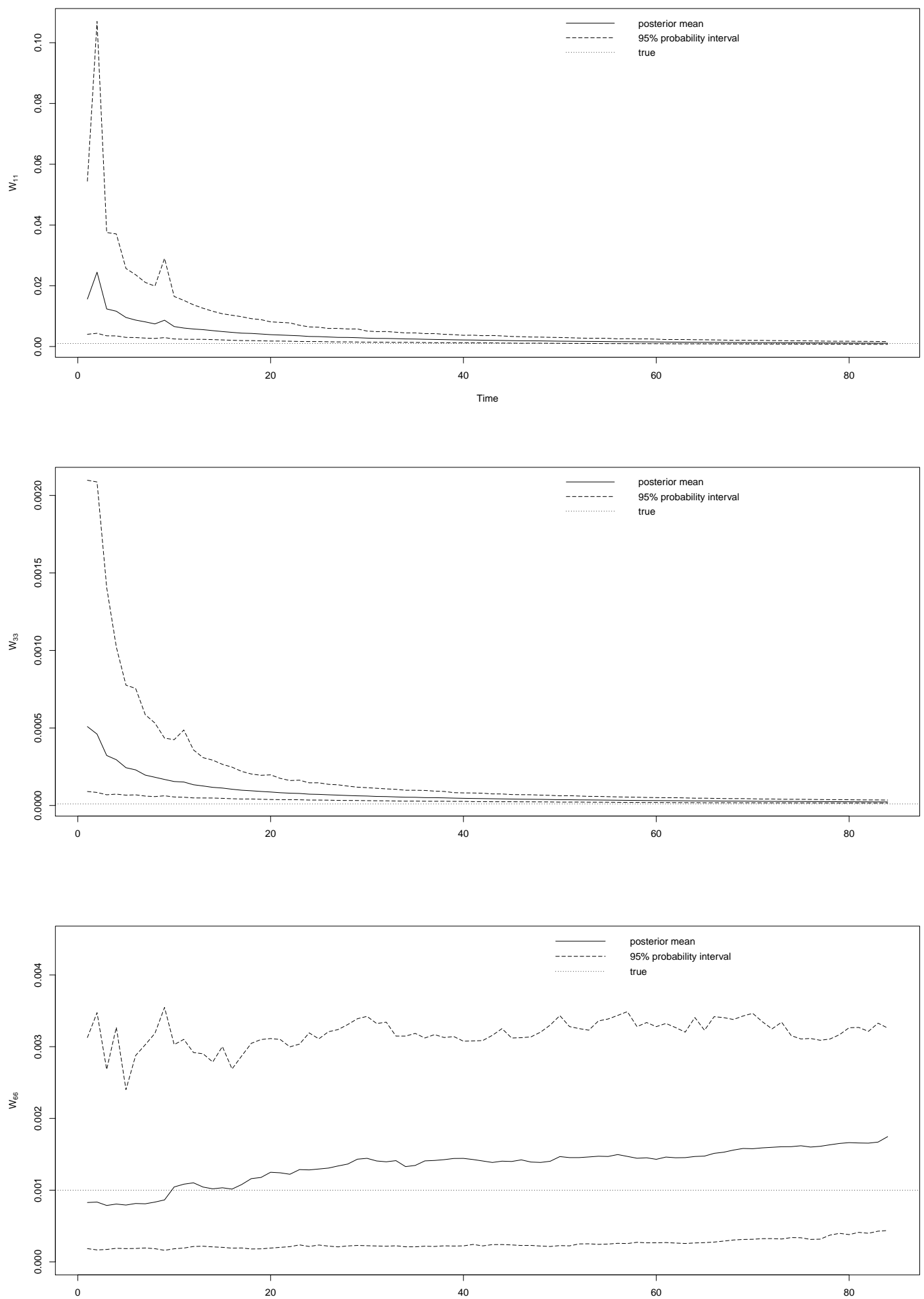

Figura 6.28: Gráfico das principais componentes da matriz de variância $W$ estimados no modelo BIZU. 

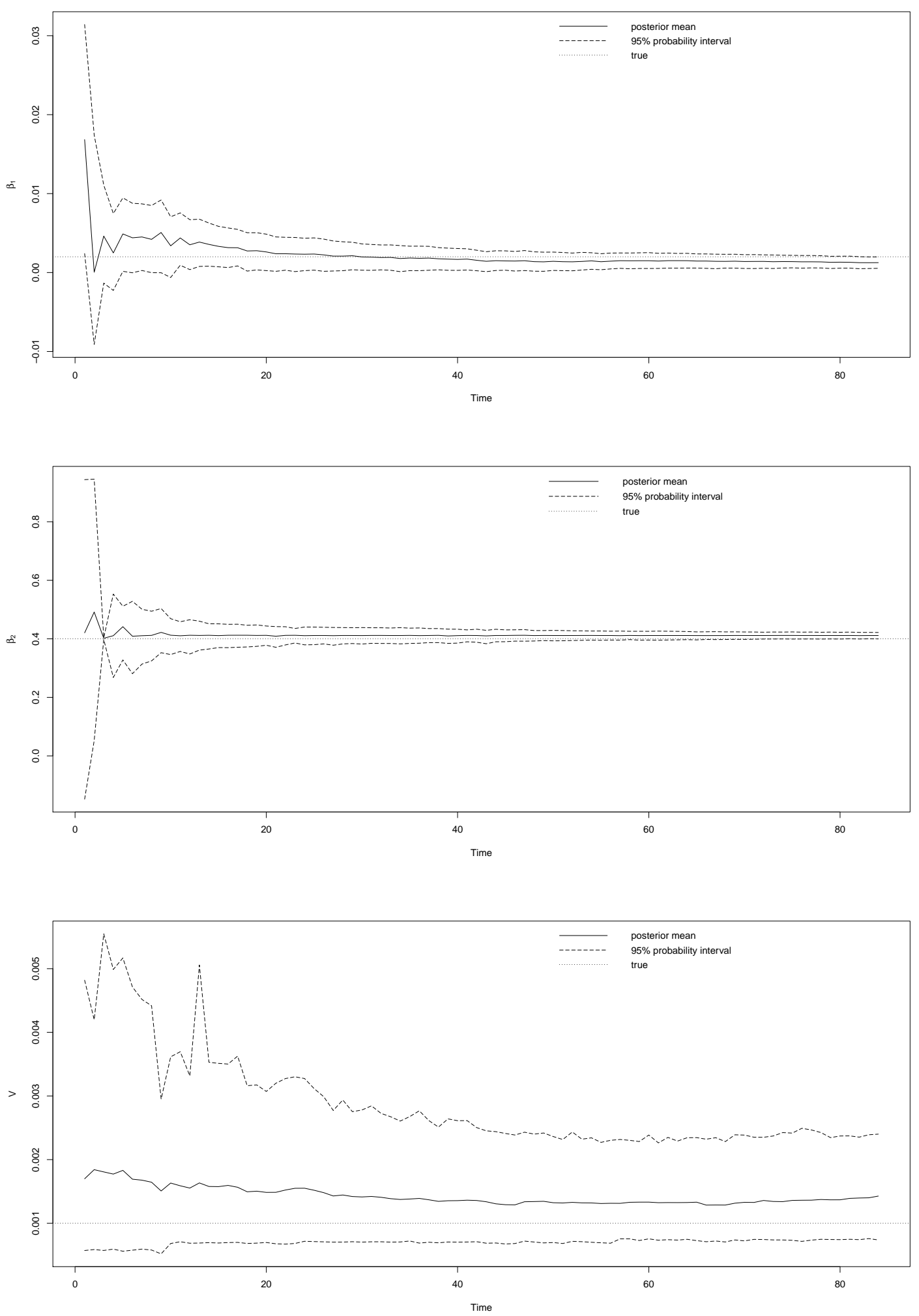

Figura 6.29: Gráfico da estimativa das parâmetros das covariáveis do modelo BIZU. 

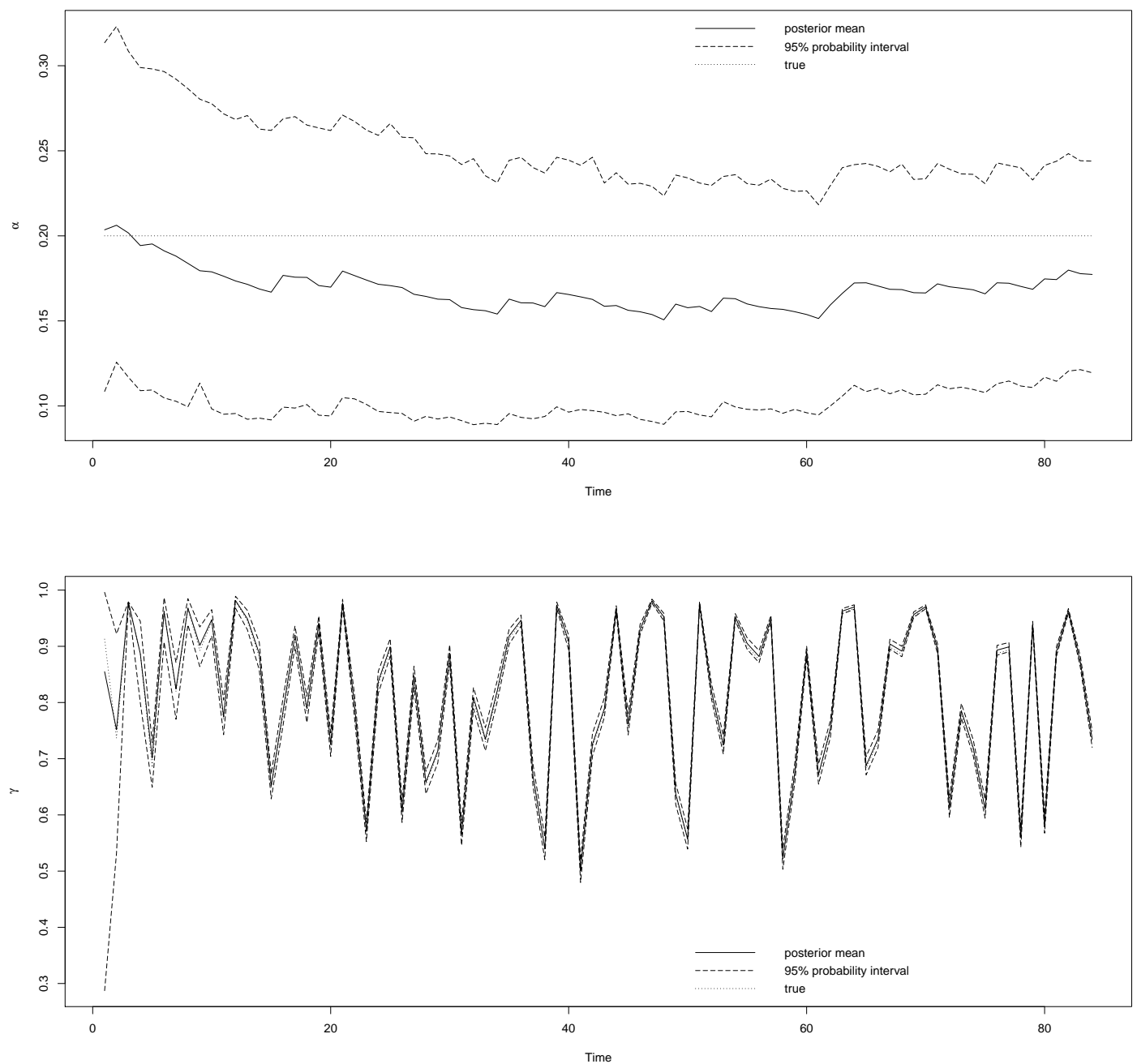

Figura 6.30: Gráfico da estimativa das probabilidades $\alpha$ e $\gamma$ do modelo BIZU.

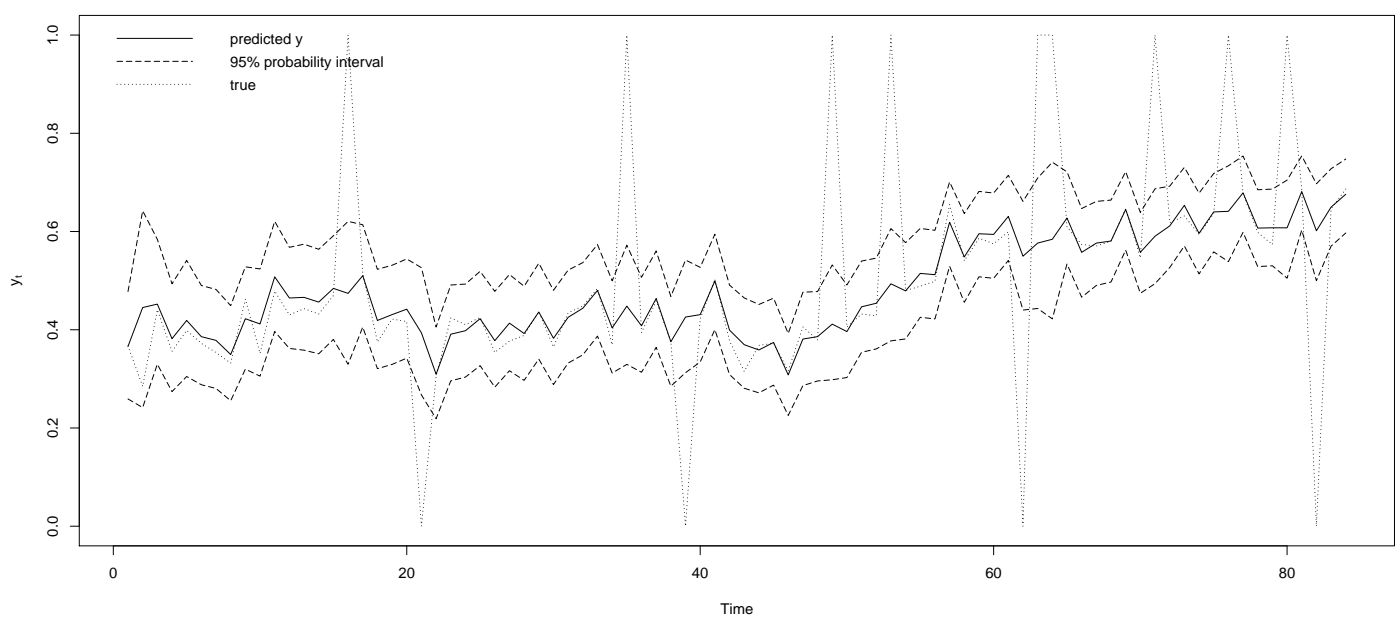

Figura 6.31: Gráfico da distribuição preditiva $p\left(y_{t} \mid D_{t-1}\right)$ da componente contínua do modelo dinâmico $B I Z U$. 


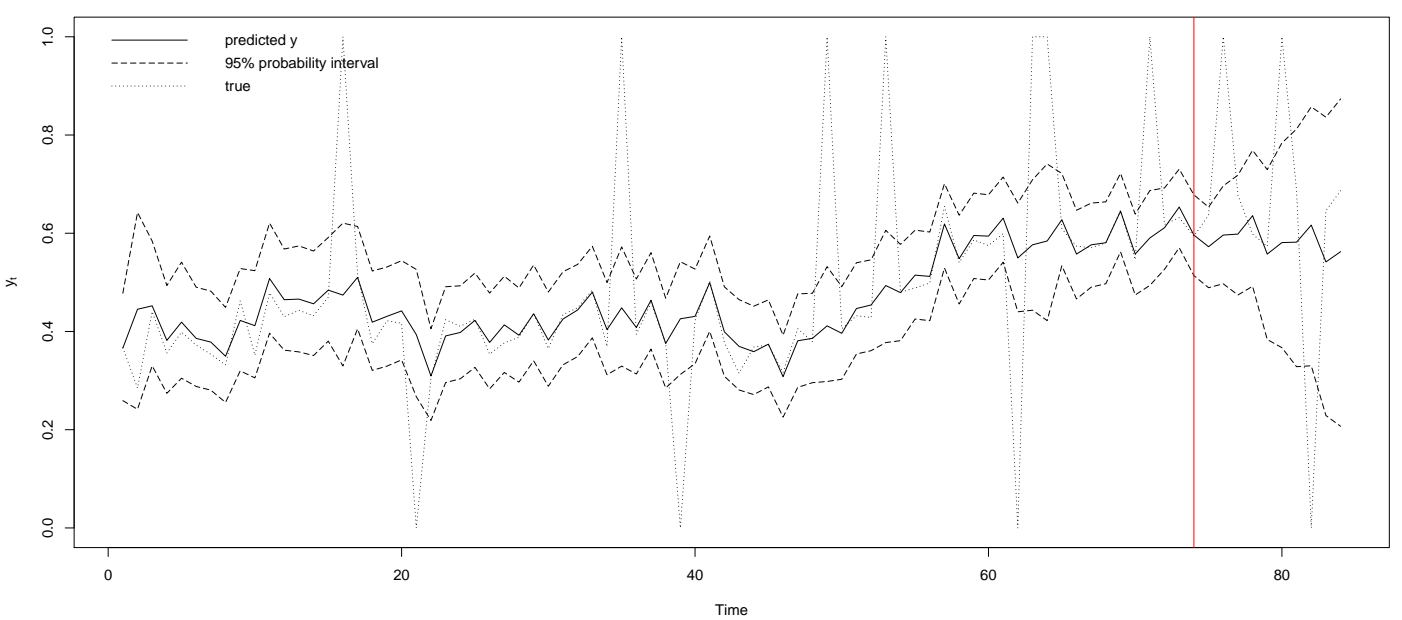

Figura 6.32: Gráfico da distribuição preditiva $p\left(y_{t} \mid D_{t-1}\right)$ da componente contínua do modelo dinâmico $B I Z U$ com previsão a 10 passos a frente. 


\subsection{Estudo de simulação para o modelo dinâmico Tobit}

Considerando a estrutura do modelo apresentada na seção (4.3.1), temos os espaços de estados representados por $\boldsymbol{\theta}$ e o conjunto de parâmetros estáticos desconhecidos é dado apenas pela matriz $\boldsymbol{W}$.

Como foi descrito na seção (4.3.1), é possível obter as posterioris condicionais completas de $\boldsymbol{W}$ até um tempo presente $t$ :

$$
p\left(\boldsymbol{\psi}_{1} \mid \boldsymbol{\theta}_{1: t}, \boldsymbol{y}_{1: t}\right) \sim W\left(t / 2+\nu_{1}, S S_{\theta_{1: t}}+\boldsymbol{S}_{1}\right),
$$

$\operatorname{com} S S_{\theta_{t}}=\left(\boldsymbol{\theta}_{t}-\boldsymbol{G}_{t} \boldsymbol{\theta}_{t-1}\right)\left(\boldsymbol{\theta}_{t}-\boldsymbol{G}_{t} \boldsymbol{\theta}_{t-1}\right)^{\prime}$ e $S S_{\theta_{1: t}}=\sum_{i=1}^{t} S S_{\theta_{i}}$.

Desta forma o conjunto de estatísticas suficientes dada as observações até o tempo $t$ necessário para atualização das posterioris condicionais completas até o tempo presente:

$$
\left(S S_{\theta_{1: t}}\right) .
$$

Além disso, para o filtro auxiliar precisamos obter amostras $\mu_{t}^{k}$ como uma função de $p\left(\boldsymbol{\theta}_{t} \mid \boldsymbol{\theta}_{t-1}^{k}, \boldsymbol{\Theta}^{k}\right)$, $k=1, \ldots, N$. Pela estrutura linear do modelo é natural escolhermos a média, ou seja:

$$
\mu_{t}=E\left(\boldsymbol{\theta}_{t} \mid \boldsymbol{\theta}_{t-1}, \boldsymbol{\Theta}\right)
$$

Assim, podemos amostrar:

$$
\mu_{t}^{k} \sim N\left(G \boldsymbol{\theta}_{t-1}^{k}, W^{k}\right) .
$$

Tendo estas medidas definidas, para o processo de estimação via filtro de partículas basta seguir os algoritmos descritos em 5.3. Em todas as simulações deste estudo foram utilizadas o número de partículas $N=10000$.

\subsubsection{Modelo com tendência e sazonalidade}

Neste estudo, foi considerada a seguinte estrutura de modelo:

$$
\begin{aligned}
& \eta_{t}=F^{\prime} \theta_{t} \\
& \theta_{t}=G \theta_{t-1}+\omega, \quad \omega \sim N(0, W) .
\end{aligned}
$$

com $G$ composta por 3 matrizes bloco diagonais:

$$
J_{2}=\left(\begin{array}{ll}
1 & 1 \\
0 & 1
\end{array}\right), \quad P=\left(\begin{array}{cc}
-\mathbf{1}_{p-2} & -1 \\
\mathbf{I}_{p-2} & 1
\end{array}\right), \quad \mathbf{e} \quad I_{1}=(1) .
$$

onde $J$ representa a evolução de nível e tendência da média $\mu_{t}, P$ representa a evolução da sazonalidade de período de tamanho $p$ e $I_{1}$ corresponde a evolução de nível da precisão $\phi_{t}$. A matriz $F$ é dada por

$$
F=\left(\begin{array}{ccccc}
1 & 0 & 1 & \mathbf{0}_{p-2} & 0 \\
0 & 0 & 0 & \mathbf{0}_{p-2} & 1
\end{array}\right)
$$

Foi gerada uma série de tamanho $T=84$ e com periodicidade $p=4$. A variância do componente do erro da equação de evolução $W$ possui a mesma estrutura bloco-diagonal de $G$, o que permite que para o processo de estimação seja fragmentada em 3 sub-matrizes, permitindo a amostragem de variáveis com dimensões menores. A matriz $W$ foi fixada em $W_{1}=\operatorname{diag}(0,01,0,0001), W_{2}=$ $\operatorname{diag}\left(10^{-2}, 10^{-2}, 10^{-2}\right)$ e $W_{3}=0,001, \operatorname{com} W=\operatorname{bdiag}\left(W_{1}, W_{2}, W_{3}\right)$. Os hiperparâmetros da priori inicial para os espaços de estados foram definidos por

$$
m_{0}=(\operatorname{logit}(0,1) \times(0,9,0,01,0,08,0,005,0,005), \log (0,1))^{\prime}
$$

e $C_{0}=\operatorname{bdiag}\left(C_{01}, C_{02}, C_{03}\right), C_{01}=\operatorname{diag}(0,01,0,01), C_{02}=\operatorname{diag}\left(10^{-4}, 10^{-4}, 10^{-4}\right)$ e $C_{03}=0,001$, 
$\theta_{0} \sim N\left(m_{0}, C_{0}\right)$

As prioris para as componentes da variância do erro de evolução foram dadas por $W_{01}^{-1} \sim$ $W(7 / 2, \operatorname{diag}(8,100)), W_{02}^{-1} \sim W(10 / 2, \operatorname{diag}(5,5,5))$ e $W_{03}^{-1} \sim G(5 / 2,0,001 \times 5 / 2)$, com $W_{0}=$ $\operatorname{diag}\left(W_{01}, W_{02}, W_{03}\right)$ e os hiperparâmetros para a priori do espaço de estado inicial foram $m_{0}=$ $\left(\left(\operatorname{logit}\left(y_{1}\right) \times(0,9,0,01,0,08,0,005,0,005), \log \left(d p\left(y_{1: T}\right)\right)\right)\right)^{\prime}$ e $C_{0}=\operatorname{bdiag}\left(C_{01}, C_{02}, C_{03}\right), C_{01}=$ $\operatorname{diag}(0,05,0,05), C_{02}=\operatorname{diag}\left(5 \times 10^{-4}, 5 \times 10^{-4}, 5 \times 10^{-4}\right)$ e $C_{03}=0,005$. O tempo computacional do processo foi de aproximadamente 20 minutos.

A Figura (6.34) a estimativa de $\eta_{1 t}$ e $\eta_{2 t}$, a Figura (6.35) as estimativas de $\mu_{t}$ e $\sigma_{t}$. Na Figura (6.36) podemos observar as estimativas das principais componentes da matriz de variância $\boldsymbol{W}$. A Figura (6.39) apresenta a distribuição preditiva $p\left(y_{t} \mid D_{t-1}\right)$, sendo que foram desprezados os 10 últimos elementos do conjunto de dados parar calcular a previsão a $k$-passos a frente.

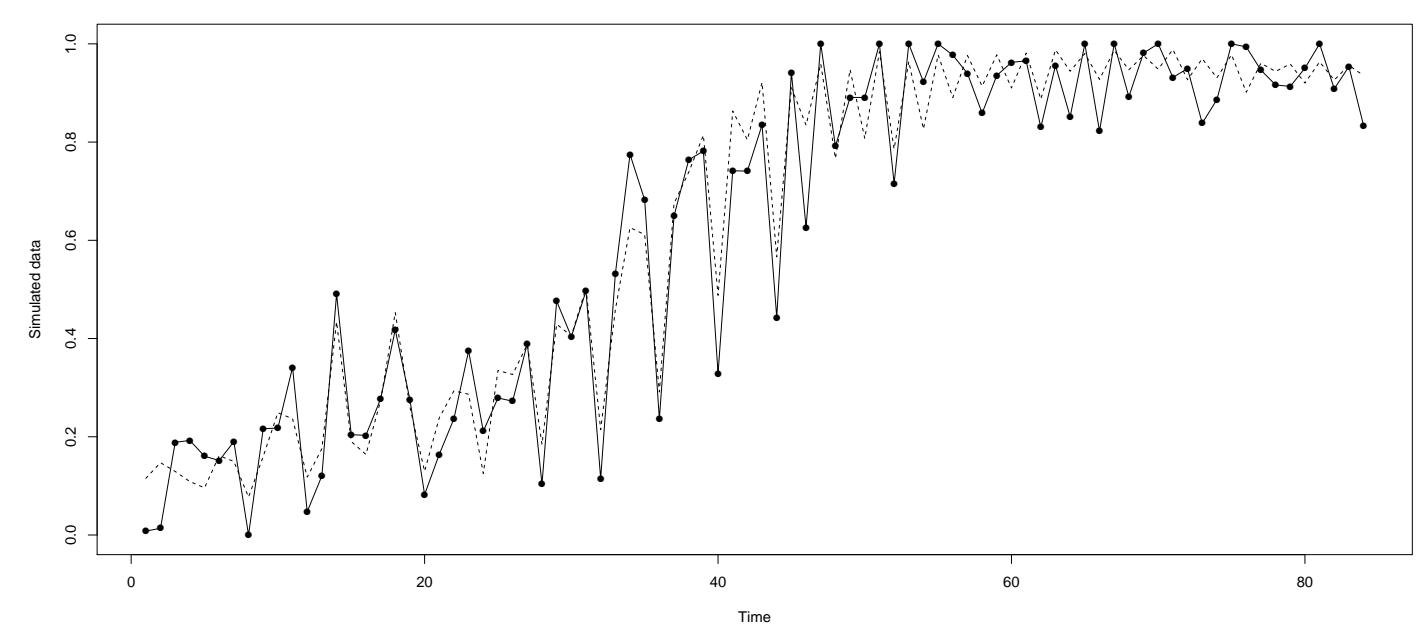

Figura 6.33: Gráfico da série simulada do modelo dinâmico Tobit com nivel, tendência e sazonalidade, com $y_{t}$ (linha continua) e $\mu_{t}$ (linha pontilhada). 

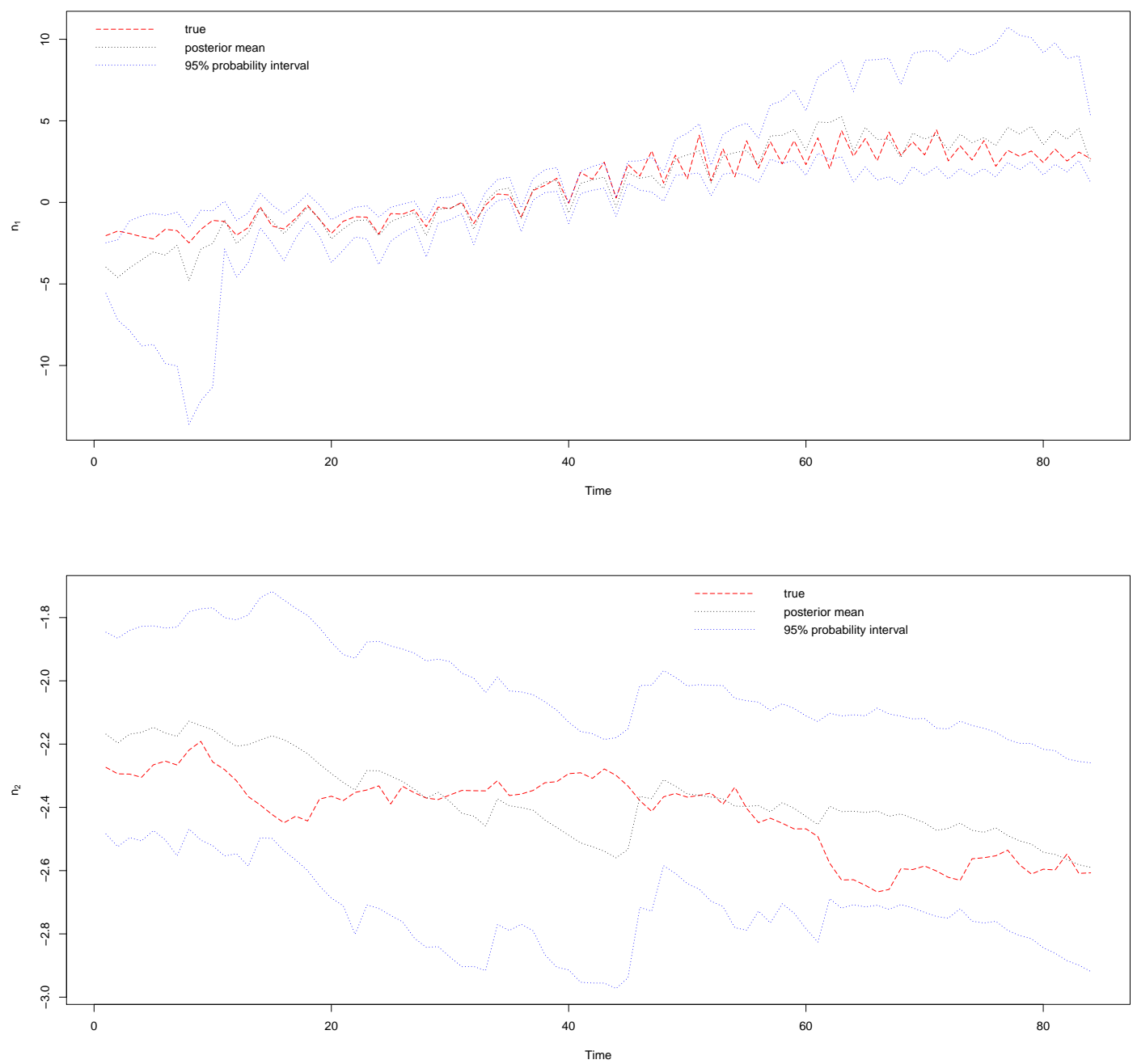

Figura 6.34: Gráfico dos preditores lineares de $\mu_{t}$ e $\sigma_{t}$ do modelo dinâmico Tobit. 

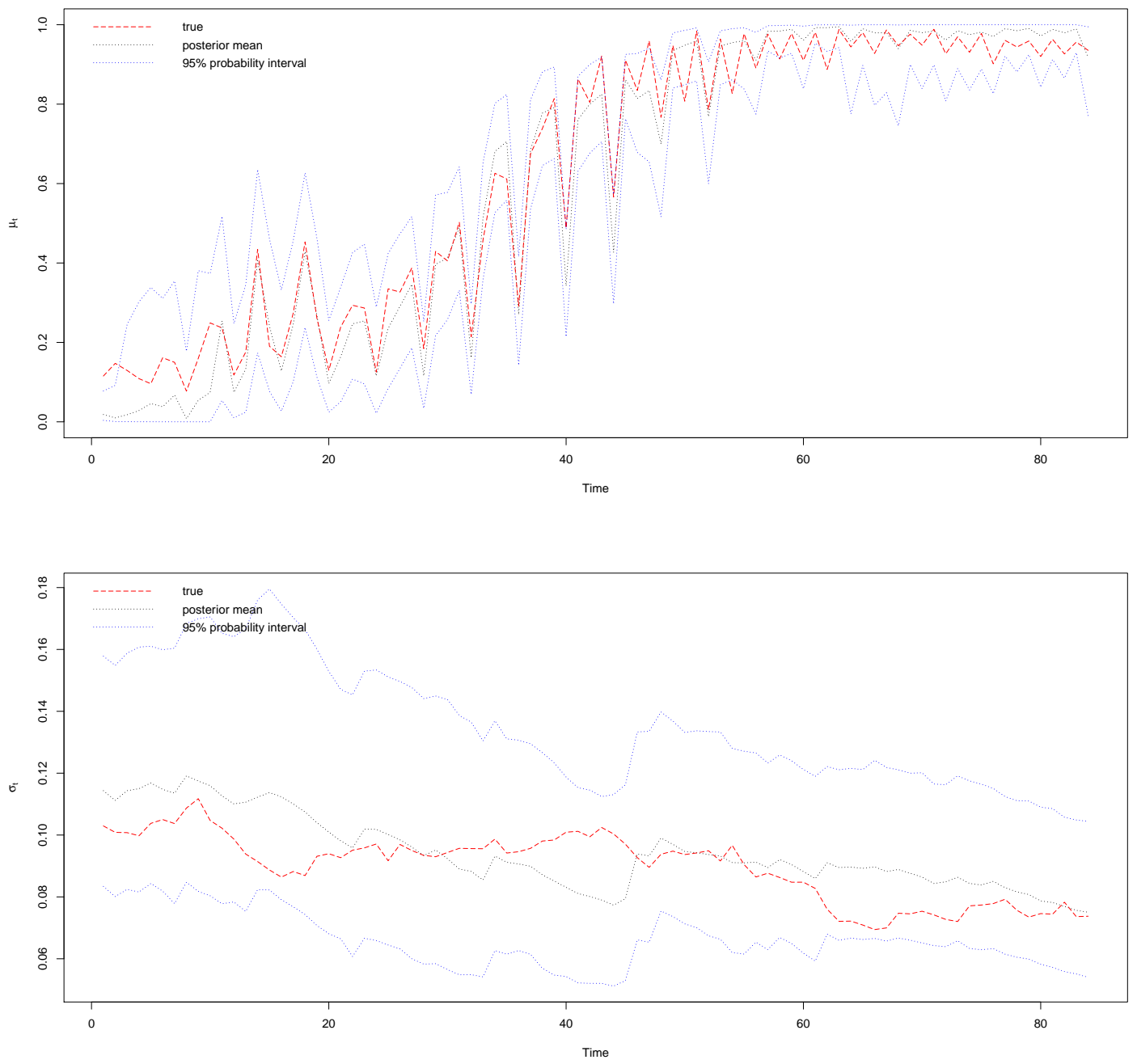

Figura 6.35: Gráfico dos estados $\mu_{t}$ e $\sigma_{t}$ do modelo dinâmico Tobit. 

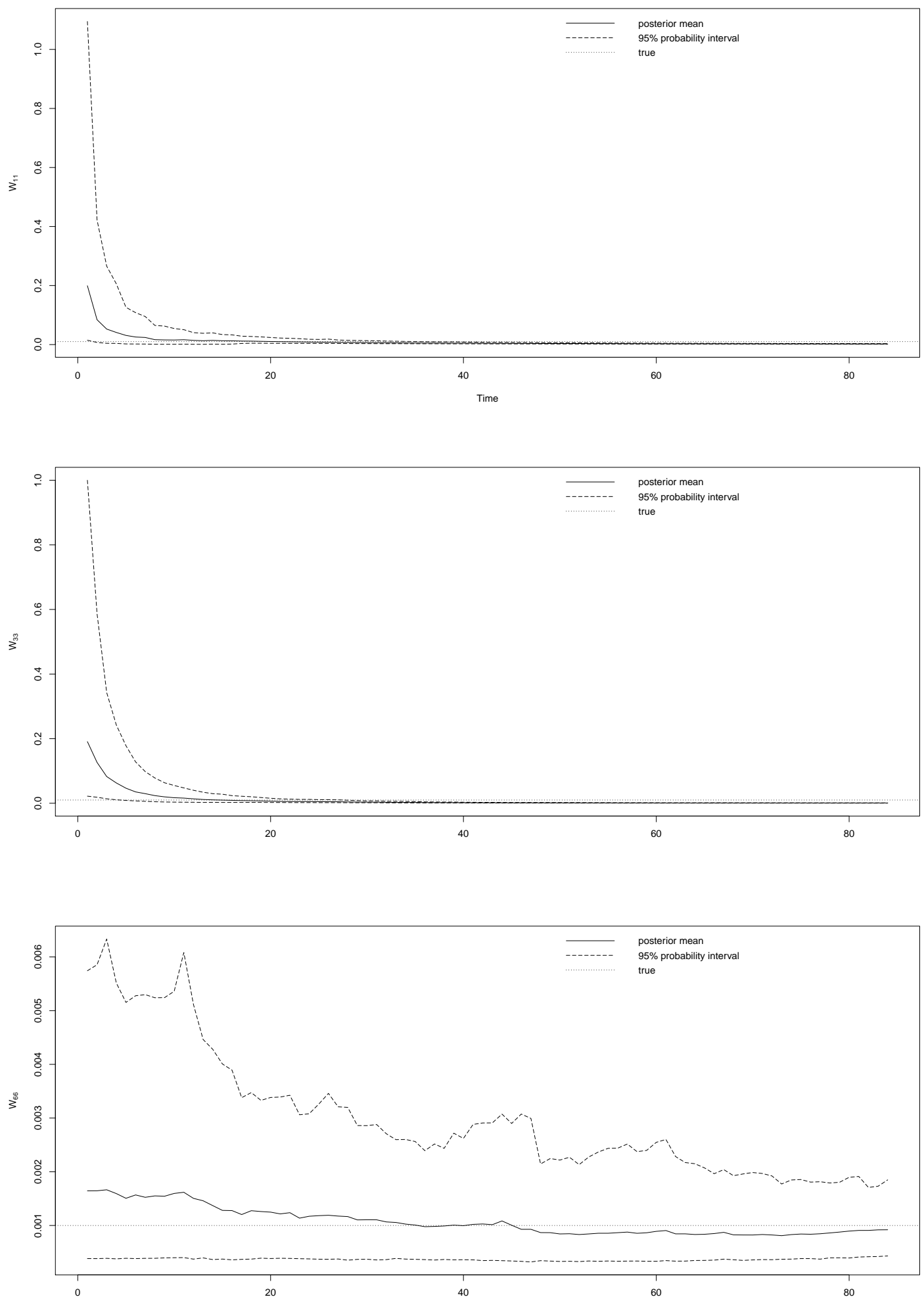

Figura 6.36: Gráfico das principais componentes da matriz de variância $W$ estimados no modelo Tobit. 

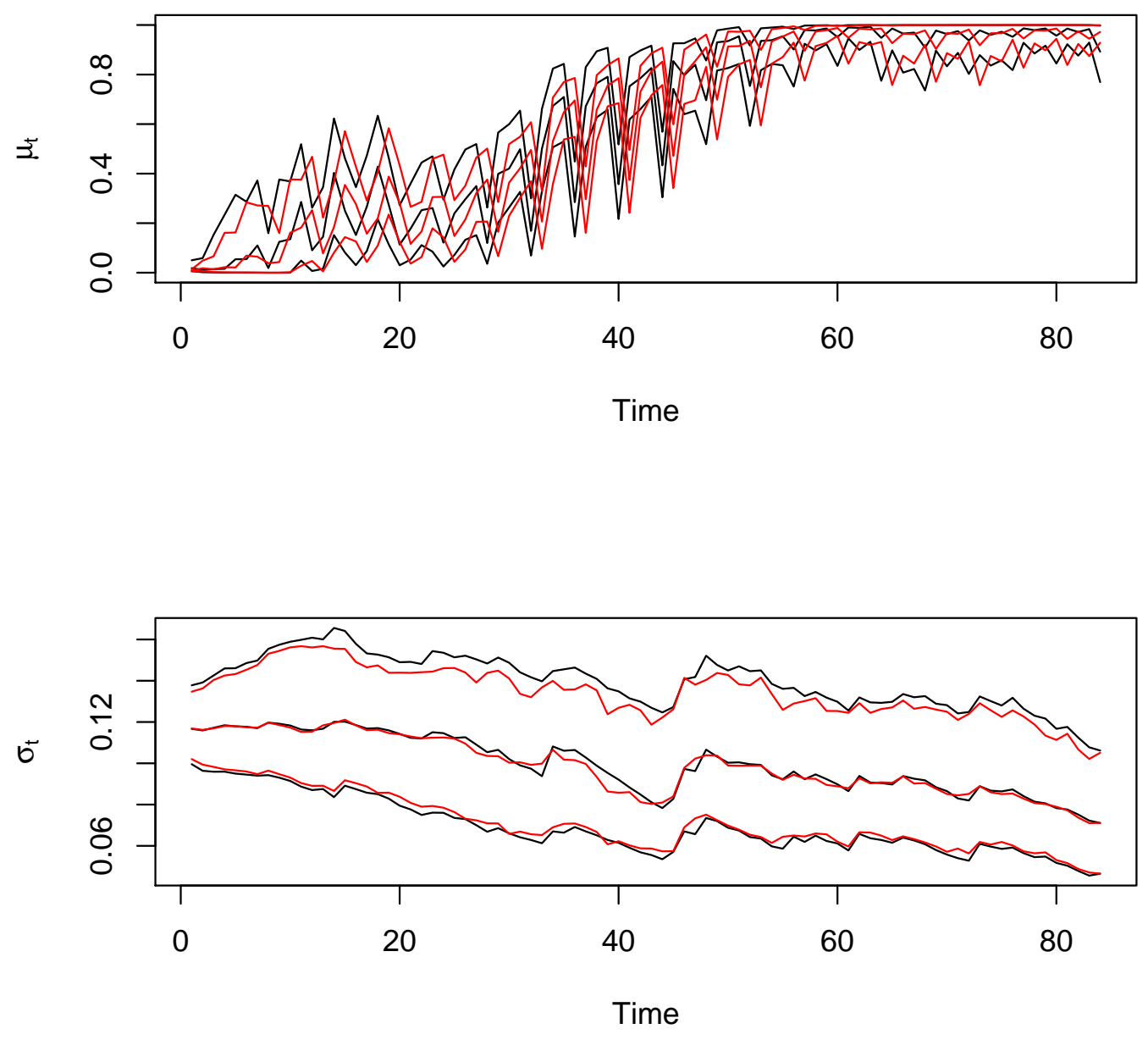

Figura 6.37: Gráfico dos preditores lineares suavizados em comparação ao estimados no processo de filtragem.

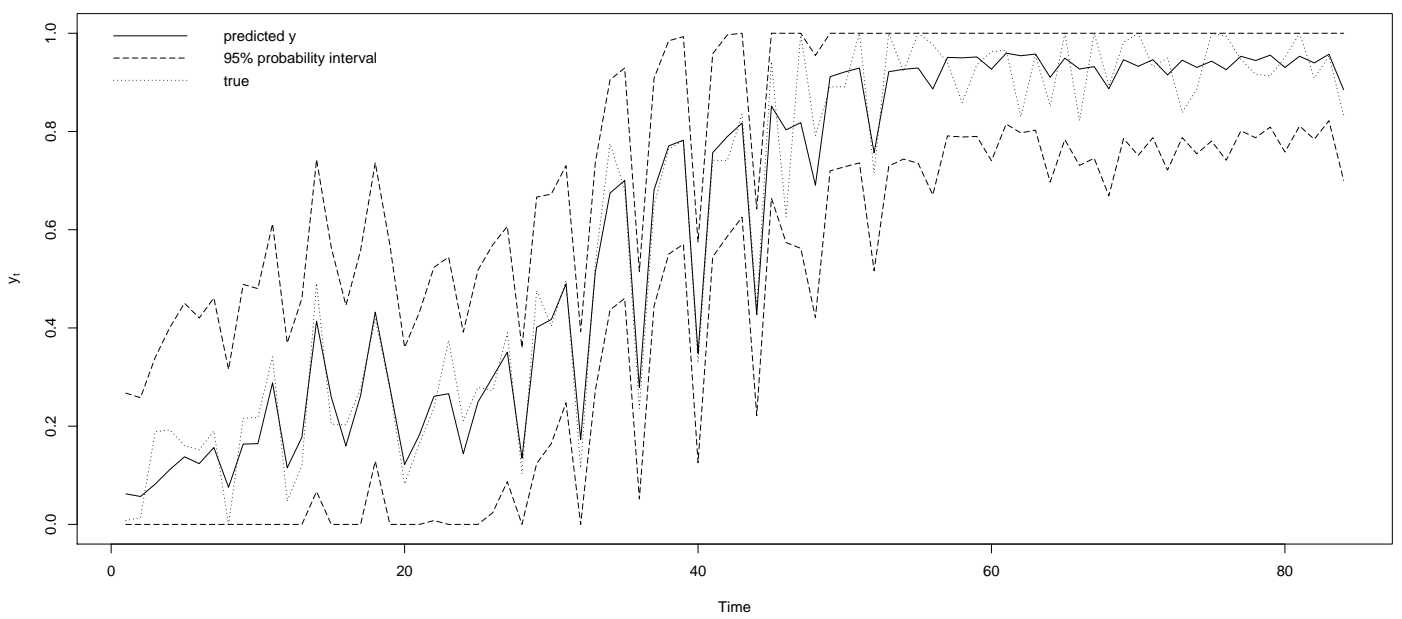

Figura 6.38: Gráfico da distribuiçãa preditiva $p\left(y_{t} \mid D_{t-1}\right)$ do modelo dinâmico Tobit. 


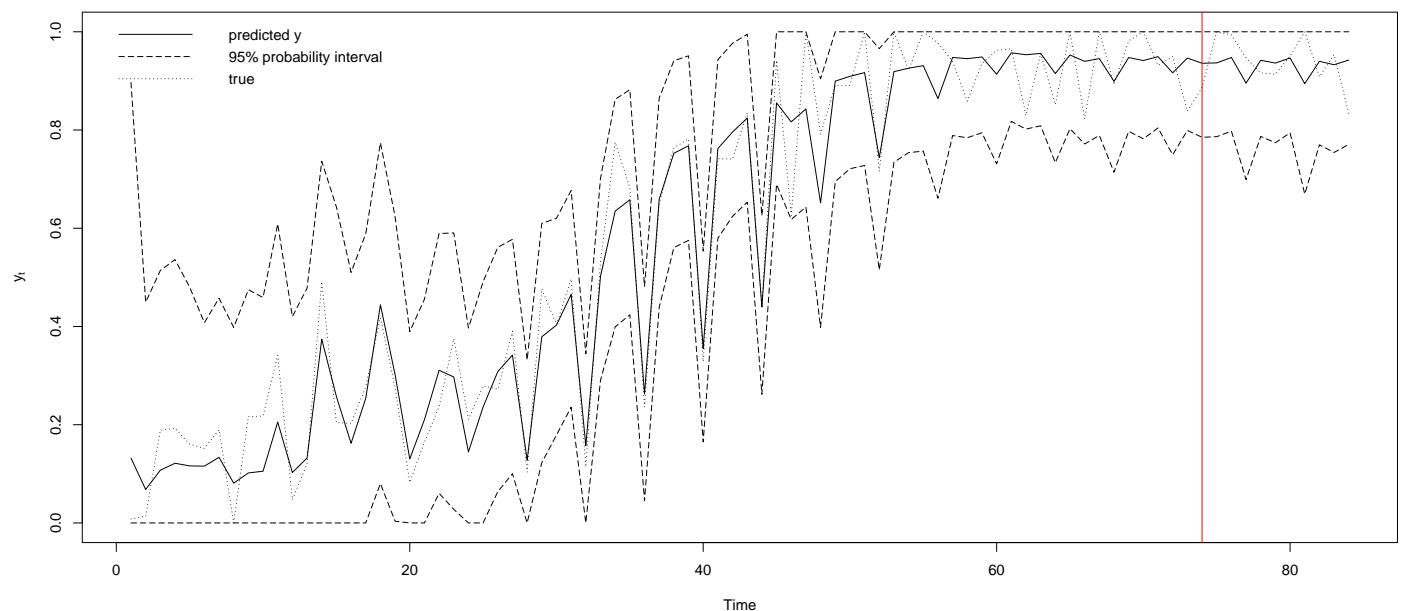

Figura 6.39: Gráfico da distribuiçãa preditiva $p\left(y_{t} \mid D_{t-1}\right)$ do modelo dinâmico Tobit com previsão a 10 passos a frente. 


\subsubsection{Modelo com covariáveis}

Neste estudo, foi considerada a seguinte estrutura de modelo:

$$
\begin{aligned}
& \eta_{t}=F^{\prime} \theta_{t} \\
& \theta_{t}=G \theta_{t-1}+\beta_{1} x_{1 t}+\omega, \quad \omega \sim N(0, W)
\end{aligned}
$$

com

$$
G=\left(\begin{array}{ccccc}
\alpha & 1 & \mathbf{0}_{p-2} & 0 & 0 \\
1 & 0 & \mathbf{0}_{p-2} & 0 & 0 \\
0 & 0 & -\mathbf{1}_{(p-2)}^{\prime} & -1 & 0 \\
\mathbf{0}_{p-2}^{\prime} & \mathbf{0}_{p-2}^{\prime} & \mathbf{I}_{(p-2)} & 0 & 0 \\
0 & 0 & \mathbf{0}_{p-2} & 0 & 1
\end{array}\right)
$$

e $F$ descritas como em (6.2.1). Neste caso, temos que considerar mais algumas estatísticas suficientes para o filtro PL, uma vez que o conjunto de parâmetros estático é dado por $(\beta, \gamma, V, W)$. Considerando as prioris:

$$
\begin{aligned}
& \alpha \sim N\left(\alpha_{0}, V_{0}\right) \\
& \beta \sim N\left(b_{0}, B_{0}\right) .
\end{aligned}
$$

Temos as posterioris condicionais completas até o tempo $t$ dadas por:

$$
\begin{aligned}
\left(\alpha \mid \theta_{1: t}, y_{1: t}, \beta, W\right) & \sim N\left(\left(\frac{\sum_{i=1}^{t} \theta_{1, i-1}\left(\theta_{1, i}-\beta_{1} x_{1 i}\right)}{W_{11}}+\frac{\alpha_{0}}{V_{0}}\right) V^{-1}, V\right) \\
\left(\beta \mid \theta_{1: t}, y_{1: t}, \alpha, W\right) & \sim N\left(\left(\sum_{i=1}^{t} x_{i}\left(\theta_{i}-G \theta_{i-1}\right) W^{-1}+\beta_{0}\left(B_{0}\right)^{-1}\right) B^{-1}, B\right) .
\end{aligned}
$$

com $V=\left(\sum_{i=1}^{t} \theta_{1, i-1}^{2}\right) / W_{11}+1 / V_{0}$ e $B=\left(\sum_{i=1}^{t} x_{i}^{2}\right) W^{-1}+B_{0}^{-1}$. Neste caso, temos o conjunto de estatísticas suficientes dado por

$$
\left(\sum_{i=1}^{t} x_{i}^{2}, \sum_{i=1}^{t} x_{i}\left(\theta_{i}-G \theta_{i-1}\right), \sum_{i=1}^{t} \theta_{1, i-1}^{2}, \sum_{i=1}^{t} \theta_{1, i-1}\left(\theta_{1 i}-\beta_{1} x_{1 i}\right), S S_{\theta_{1: t}}\right) .
$$

Foi gerada uma série de tamanho $T=84$, periodicidade $p=4$, parâmetros $\alpha=0,5$ e $\beta=$ $\left(0,1, \mathbf{0}_{4}^{\prime},-0,1\right)$. A matriz $W$ foi fixada em $W_{1}=\operatorname{diag}(0,01,0,0001), W_{2}=\operatorname{diag}\left(10^{-2}, 10^{-2}, 10^{-2}\right)$ e $W_{3}=0,01$, com $W=\operatorname{bdiag}\left(W_{1}, W_{2}, W_{3}\right)$. Os hiperparâmetros da priori inicial para os espaços de estados foram definidos por $m_{0}=(\operatorname{logit}(0,1) \times(0,87,0,01,0,1,0,01,0,01), \log (0,1))^{\prime} \mathrm{e}$ $C_{0}=\operatorname{bdiag}\left(C_{01}, C_{02}, C_{03}\right), C_{01}=\operatorname{diag}(0,05,0,01), C_{02}=\operatorname{diag}\left(10^{-2}, 10^{-2}, 10^{-2}\right)$ e $C_{03}=0,01$, $\theta_{0} \sim N\left(m_{0}, C_{0}\right)$.

As prioris dos parâmetros relacionados as covariáveis foram dados por $\beta_{01} \sim N(0,15,0,01)$, $\beta_{02} \sim N(-0,05,0,01)$ e $\alpha_{0}^{-1} \sim N(0,6,0,2)$. As prioris para as componentes da variância do erro de evolução foram dadas por $W_{01}^{-1} \sim W(10 / 2, \operatorname{diag}(5,5)), W_{02}^{-1} \sim W(12 / 2, \operatorname{diag}(3,3,3))$ e $W_{03}^{-1} \sim G(4 / 2,0,013 \times 4 / 2)$, com $W_{0}=\operatorname{diag}\left(W_{01}, W_{02}, W_{03}\right)$ e os hiperparâmetros para a priori do espaço de estado inicial foram $m_{0}=\left(\left(\operatorname{logit}\left(y_{1}\right) \times(0,9,0,01,0,08,0,005,0,005), \log \left(d p\left(y_{1: T}\right)\right)\right)\right)^{\prime}$ e $C_{0}=\operatorname{bdiag}\left(C_{01}, C_{02}, C_{03}\right), C_{01}=\operatorname{diag}(0,5,0,01), C_{02}=\operatorname{diag}\left(10^{-1}, 10^{-1}, 10^{-1}\right)$ e $C_{03}=0,01$. O tempo computacional do processo foi de aproximadamente 25 minutos.

A Figura (6.41) a estimativa de $\eta_{1 t}$ e $\eta_{2 t}$, a Figura (6.42) as estimativas de $\mu_{t}$ e $\sigma_{t}$. Na Figura (6.43) podemos observar as estimativas das principais componentes da matriz de variância $W$, na Figura (6.44) apresenta a estimativa dos parâmetros $\alpha, \beta$. As Figuras (6.46) e (6.47) apresentam a distribuição preditiva $p\left(y_{t} \mid D_{t-1}\right)$, sendo que na última foram desprezados os 10 últimos elementos do conjunto de dados parar calcular a previsão a $k$-passos a frente. 


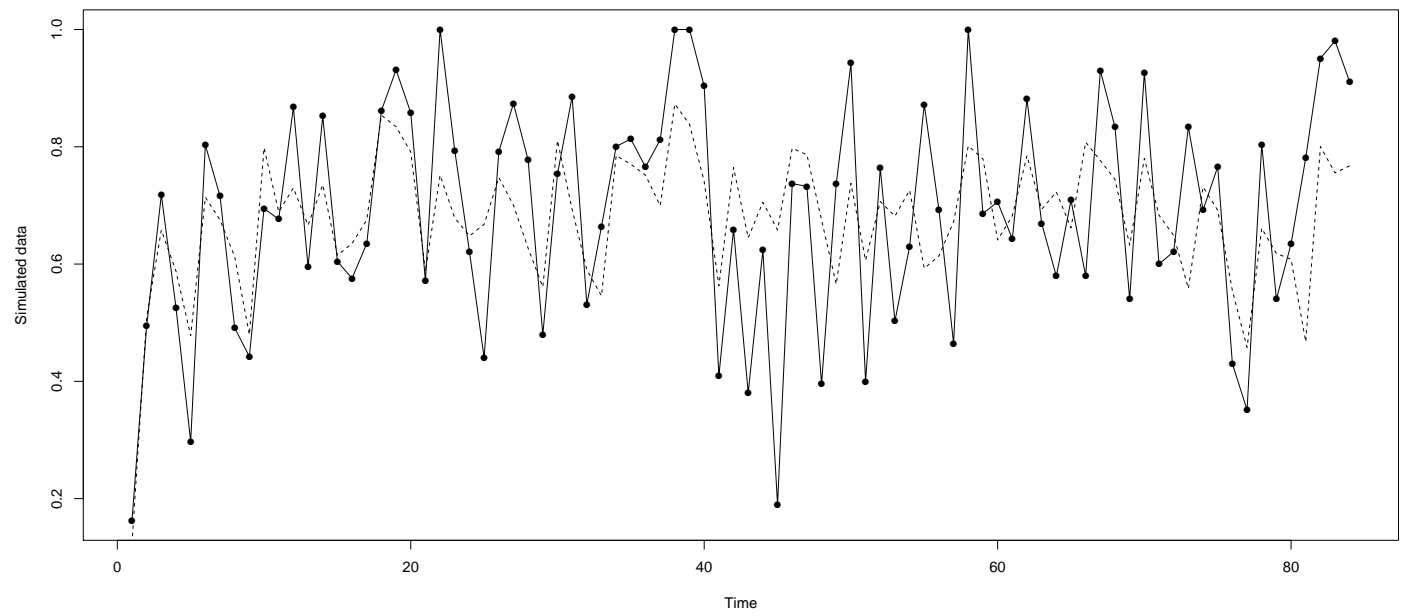

Figura 6.40: Gráfico da série simulada do modelo dinâmico Tobit com nivel, tendência e sazonalidade e covariáveis, com $y_{t}$ (linha contínua) e $\mu_{t}$ (linha pontilhada).
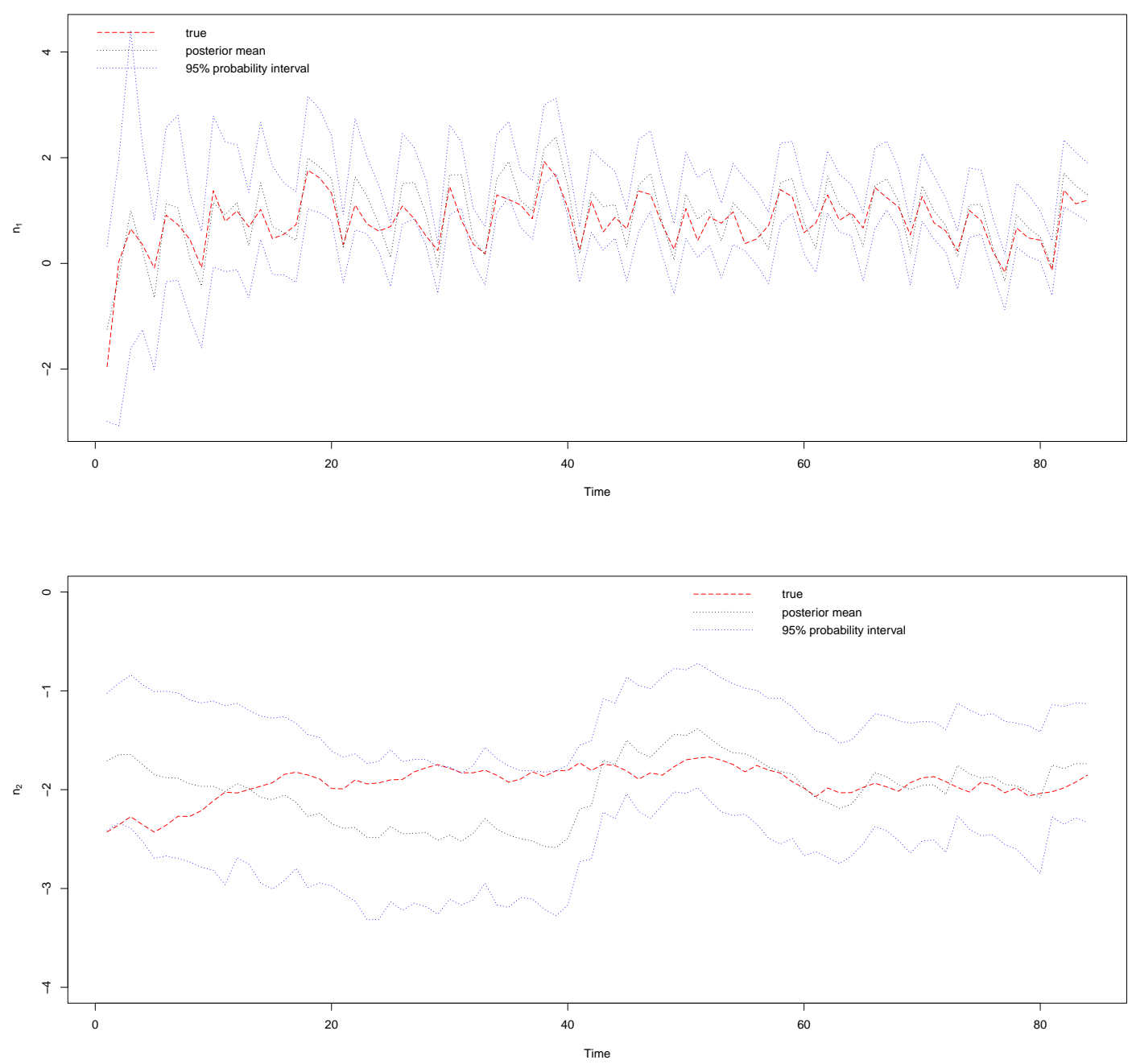

Figura 6.41: Gráfico dos preditores lineares de $\mu_{t}$ e $\sigma_{t}$ do modelo dinâmico Tobit. 

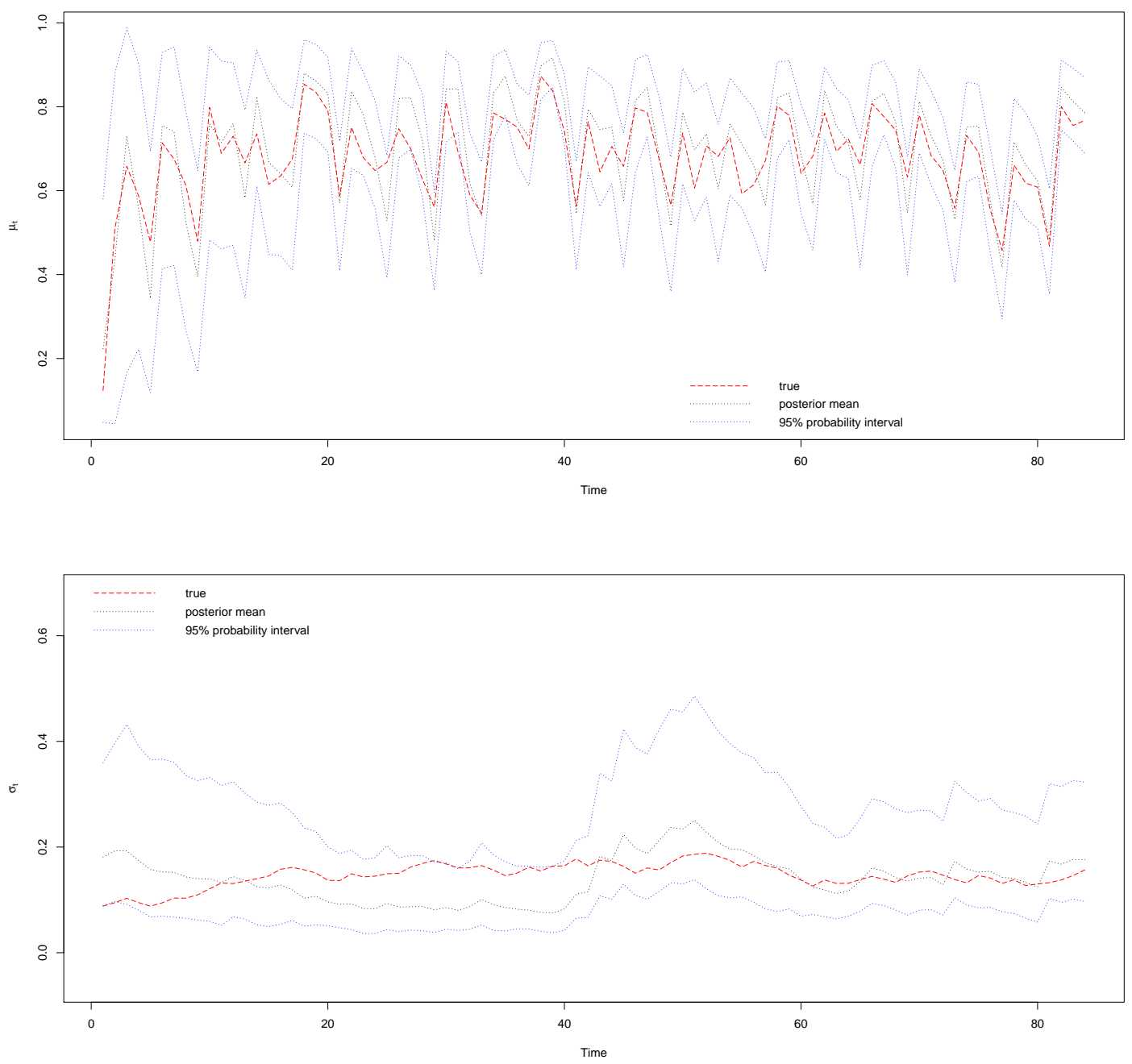

Figura 6.42: Gráfico dos estados $\mu_{t}$ e $\sigma_{t}$ do modelo dinâmico Tobit. 

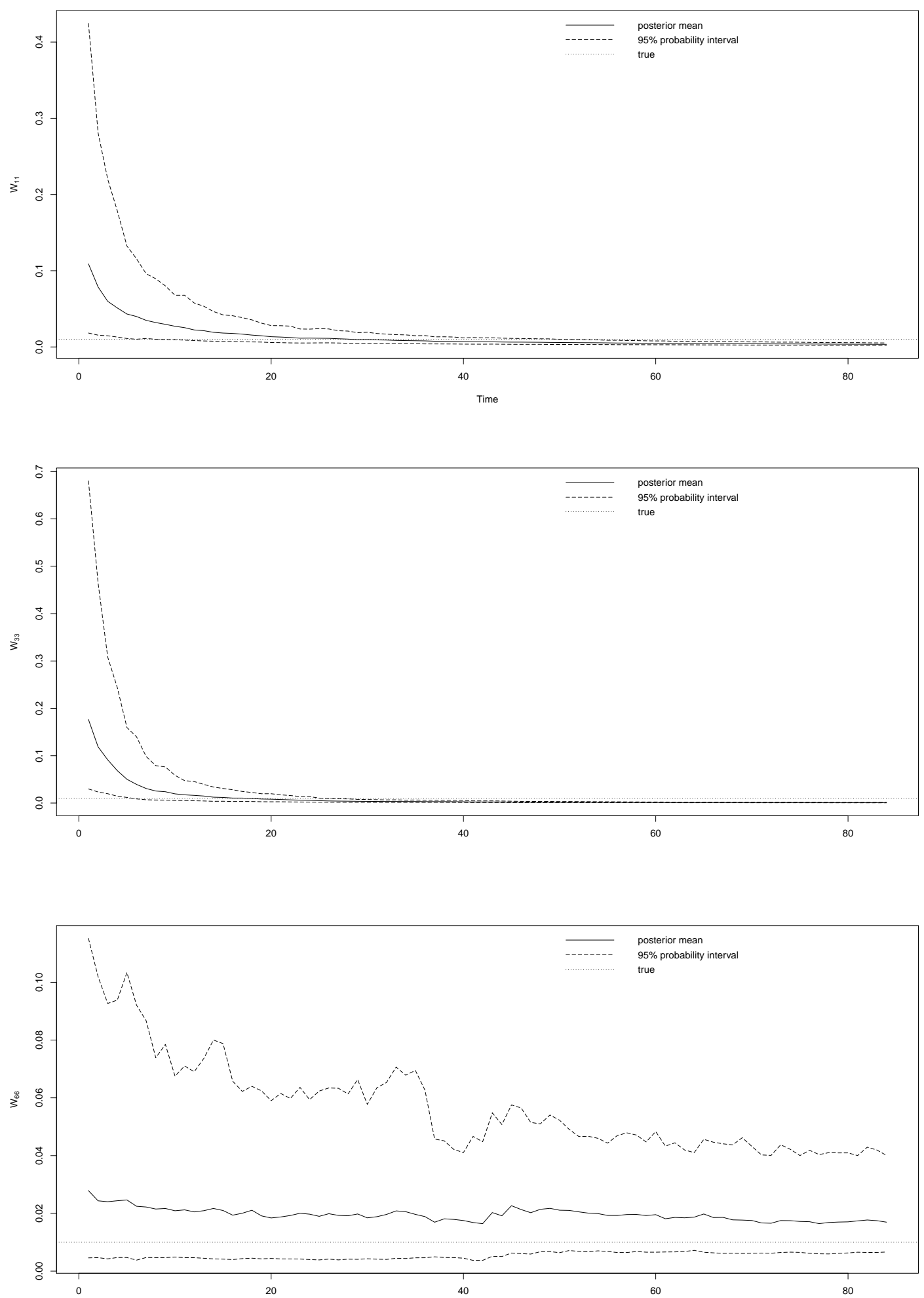

Figura 6.43: Gráfico das principais componentes da matriz de variância $W$ estimados no modelo Tobit. 

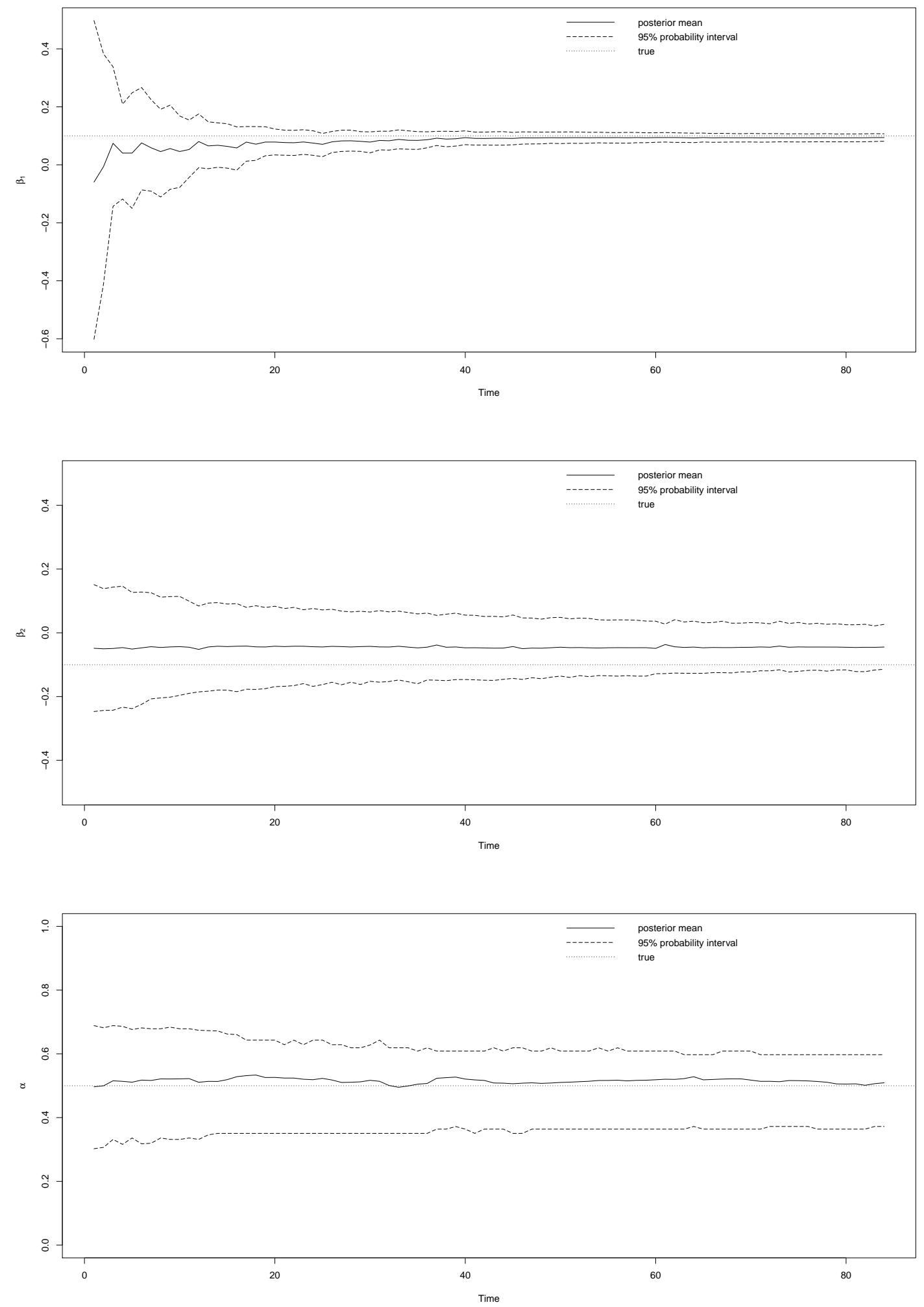

Figura 6.44: Gráfico da estimativa das parâmetros das covariáveis do modelo Tobit. 

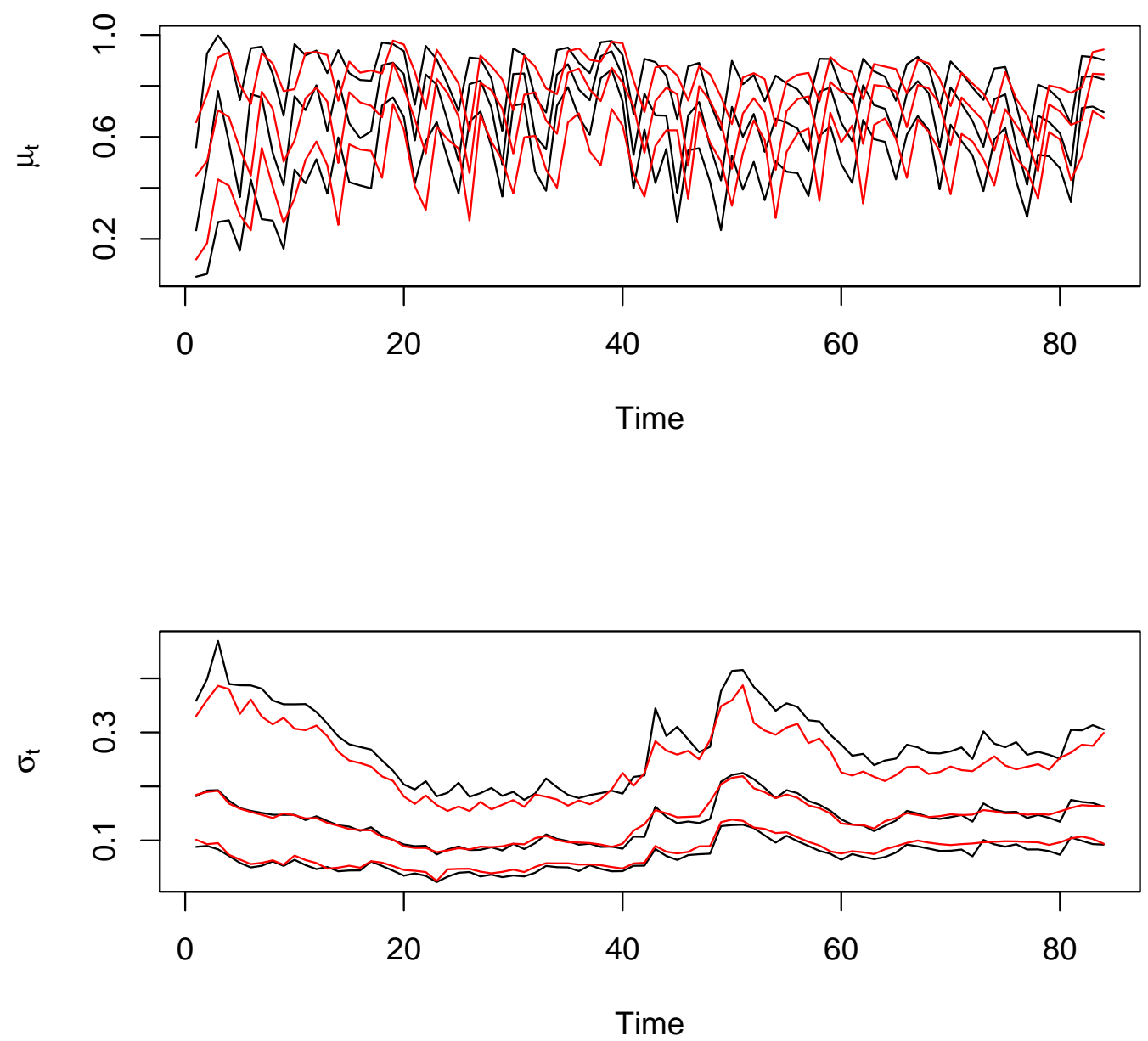

Figura 6.45: Gráfico dos preditores lineares suavizados em comparação ao estimados no processo de filtragem.

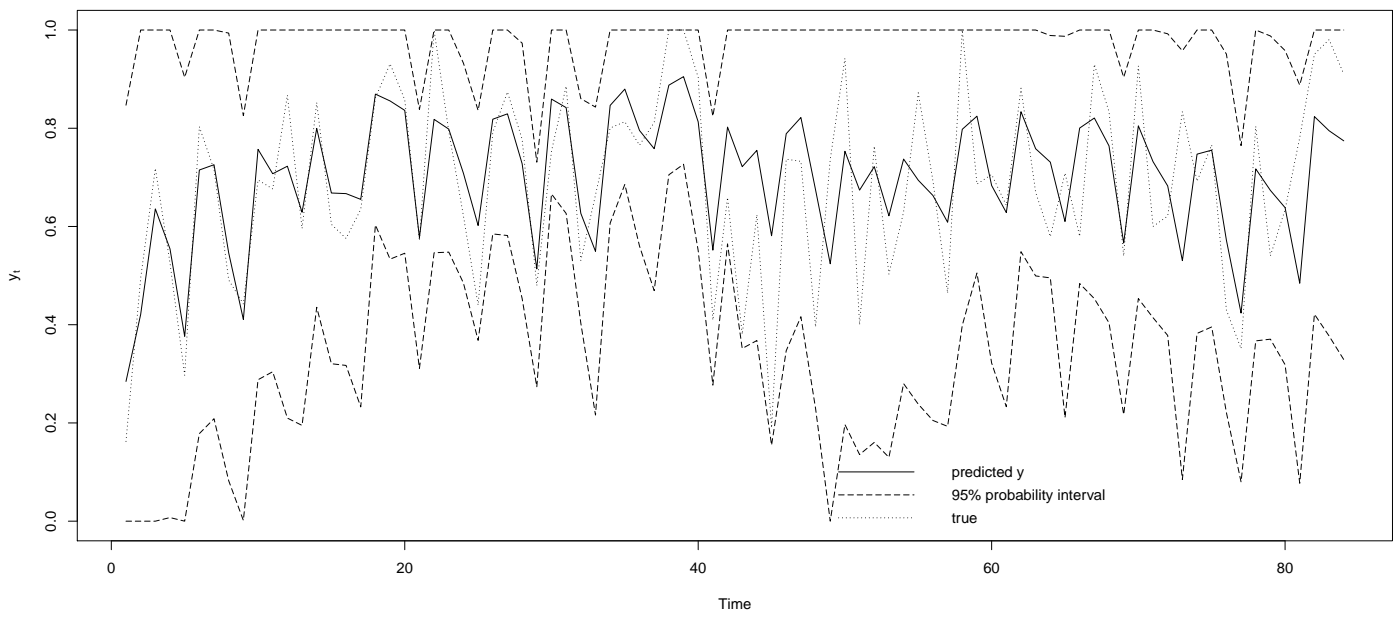

Figura 6.46: Gráfico da distribuição preditiva $p\left(y_{t} \mid D_{t-1}\right)$ do modelo dinâmico Tobit. 


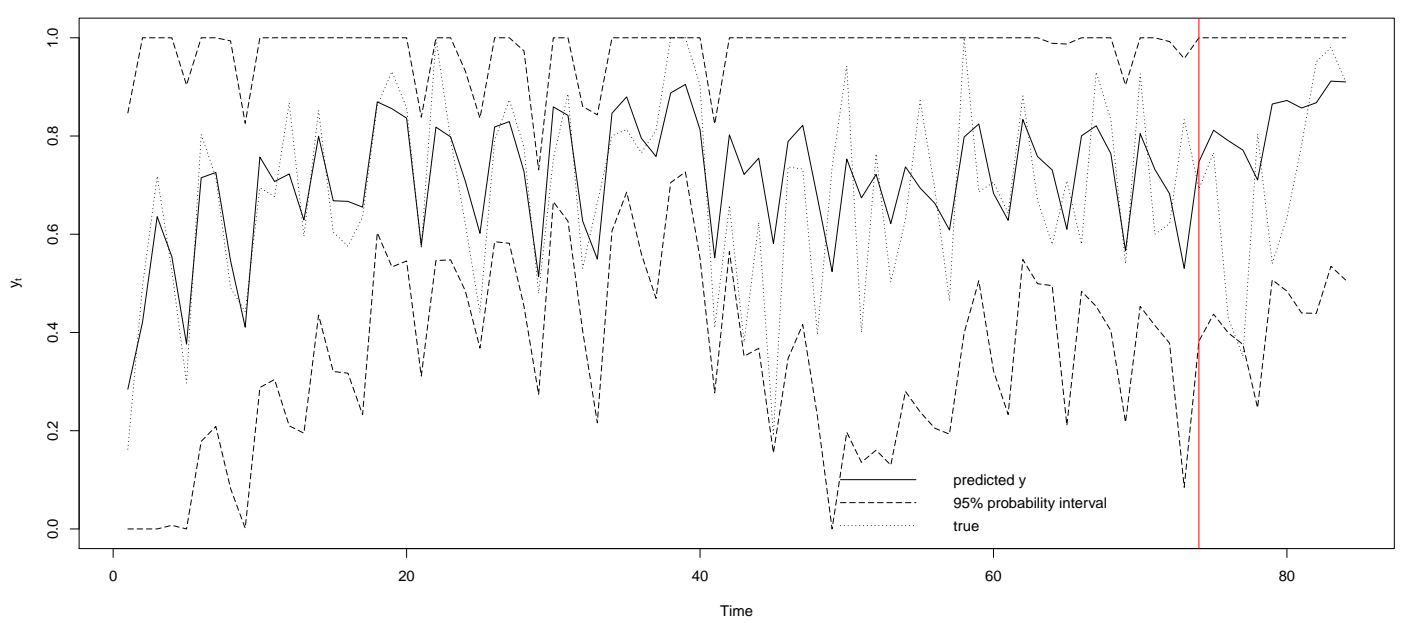

Figura 6.47: Gráfico da distribuição preditiva $p\left(y_{t} \mid D_{t-1}\right)$ do modelo dinâmico Tobit com previsão a 10 passos a frente. 


\subsection{Aplicação}

Nesta seção apresentamos uma aplicação dos modelos dinâmico BIZU e Tobit para previsão de volume de água do sistema da Cantareira. Os dados estão disponíveis no site: $<$ http://mananciais.tk $>$ como uma série de observações diárias do início de 2003 até o momento atual. No contexto do trabalho, estamos interessado em utilizar a série onde há presença de valores extremos, no caso zeros e/ou uns. Em abril de 2010 o nível de água atinge o valor total 1(100\%). No ano de 2014 o nível de água chega a baixo de 10\% no mês de maio e chega até 3\% em outubro. Alguns especialistas indicam nível do sistema abaixo de $8 \%$ como estado de extremo alerta, outro já consideram alerta para níveis abaixo de $10 \%$ conforme podemos ver nas reportagens publicadas em:

$<$ http://www.valor.com.br/brasil/3731260/justica-manda-rever-retirada-de-agua-do-sistema-cantareira $>$, $<$ http://saopa.com.br/2014/10/veja-o-que-pode-acontecer-com-o-sistema-cantareira-em-2015/> e

$<$ http://exame.abril.com.br/brasil/noticias/em-um-ano-cantareira-caiu-quase-pela-metade $>$.

O estudo de aplicação foi feito com base nos dados a partir do início do ano de 2013 até março de 2015. De acordo com este cenário, optamos por aplicar os dados considerando o nível de extremidade inferior fixado em $5 \%$, valor que representa um estado de alerta ainda mais crítico que relatado pelos especialistas. Desta forma, a série teve sua escala transformada da seguinte forma:

$$
y_{t}=\frac{\max \left(\operatorname{vol}_{t}, 0,05\right)-0,05}{1-0,05}
$$

onde vol corresponde ao vetor da série original com censura a esquerda em 0,05. Assim, a série temporal $\boldsymbol{y}$ corresponde a uma série de proporção com presença de zeros, que indicam o estado crítico de alerta no nível de água.

Como informações adicionais a série temporal, temos os níveis de água dos sistemas do Alto Tietê, Cotia, Guarapiranga, Rio Claro e Rio Grande. A Figura (6.48), disponível em

<http://noticias.uol.com.br/infograficos/2014/02/14/veja-quais-sao-os-reservatorios-de-agua-da-grande-sp.htm >,

ilustra o funcionamento do abastecimento de água dos sistemas. Para verificar a relação entre os demais sistemas com o sistema da Cantareira, as Figuras (6.50) e (6.51) mostram a relação linear e quadrática entre os sistema da cantareira com os demais. Nota-se a alta relação linear com o sistema do Alto tietê e alta relação quadrática com o sistema de Cotia. Outra variável disponível no conjunto de dados é a pluviometria mensal do sistema, no entanto, conforme apresentado na Figura (6.52), não há indícios de relação direta entre o nível do sistema com a pluviometria mensal. 


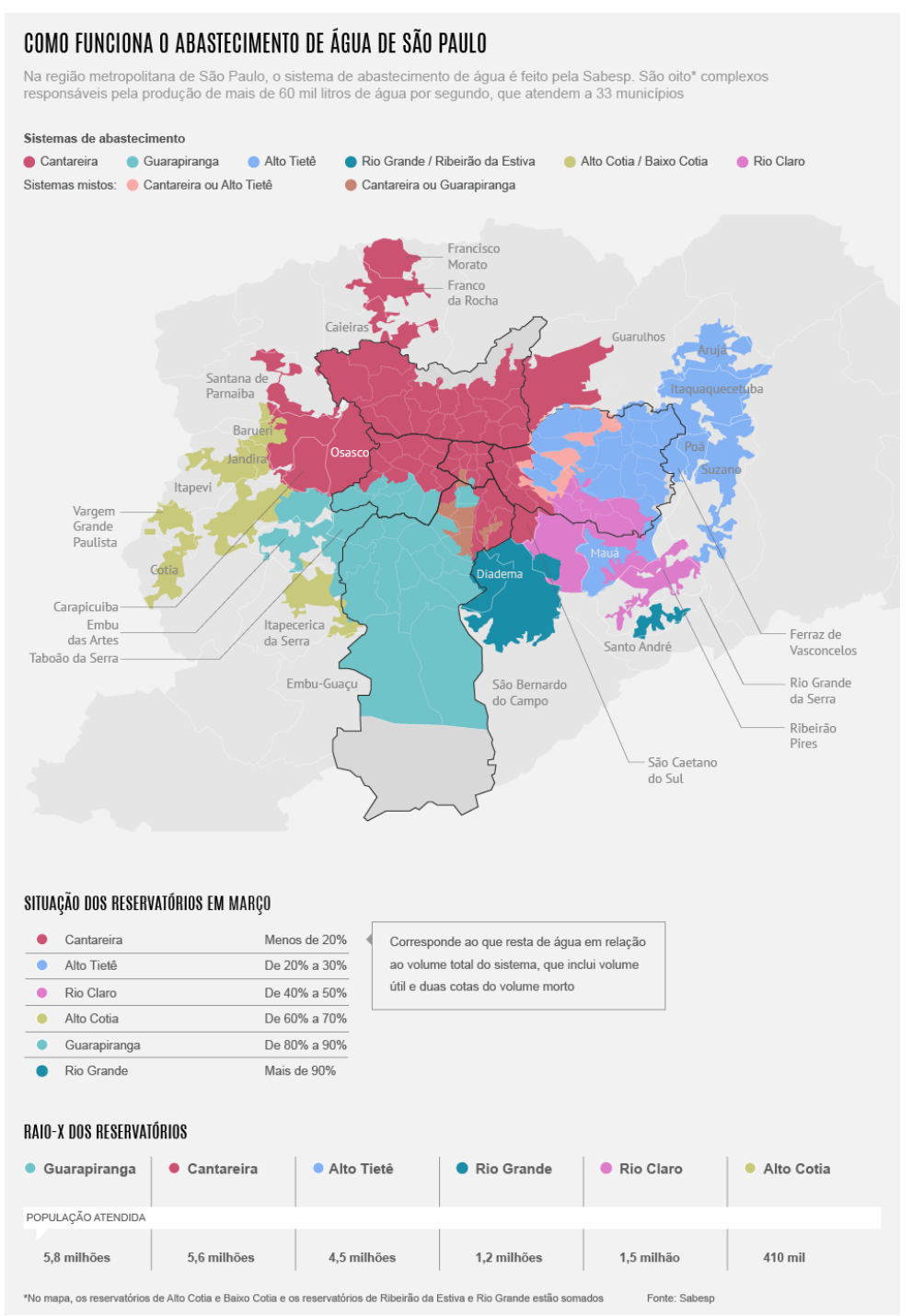

Figura 6.48: Ilustração de como funciona o sistema de abastecimento do sistema da Cantareira.

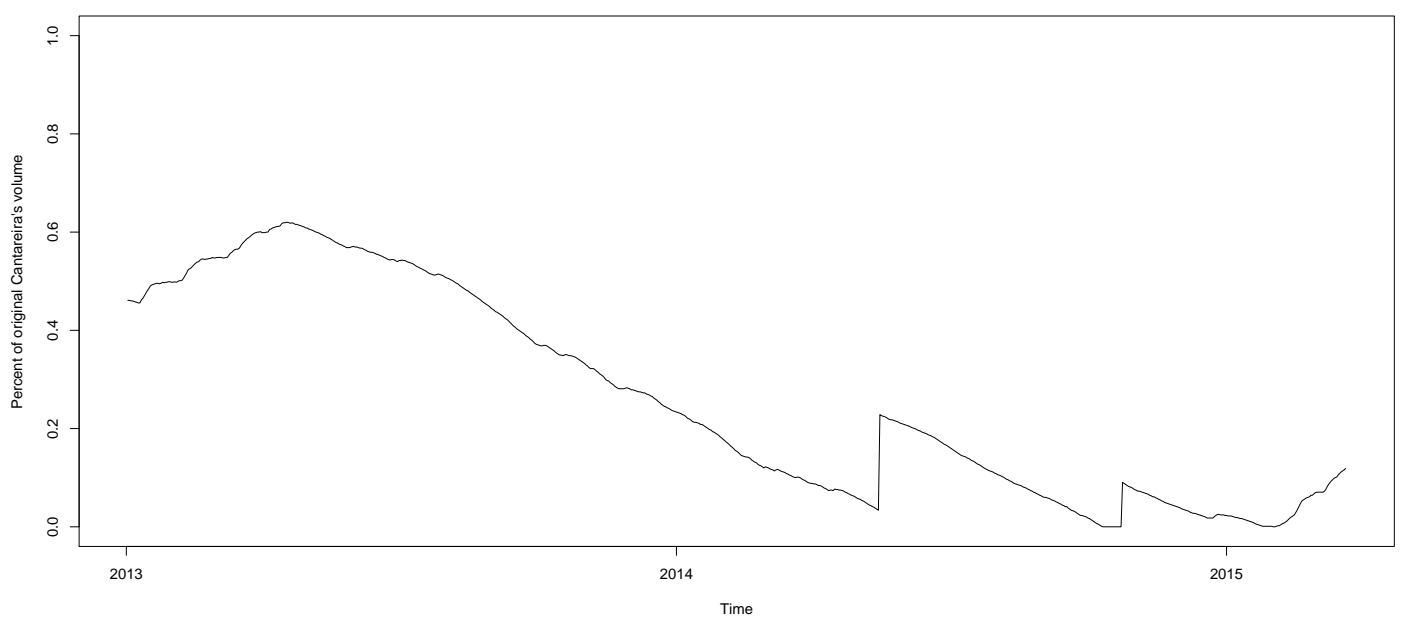

Figura 6.49: Série de dados diária do nível do sistema da Cantareira do início de 2013 a março de 2015. 

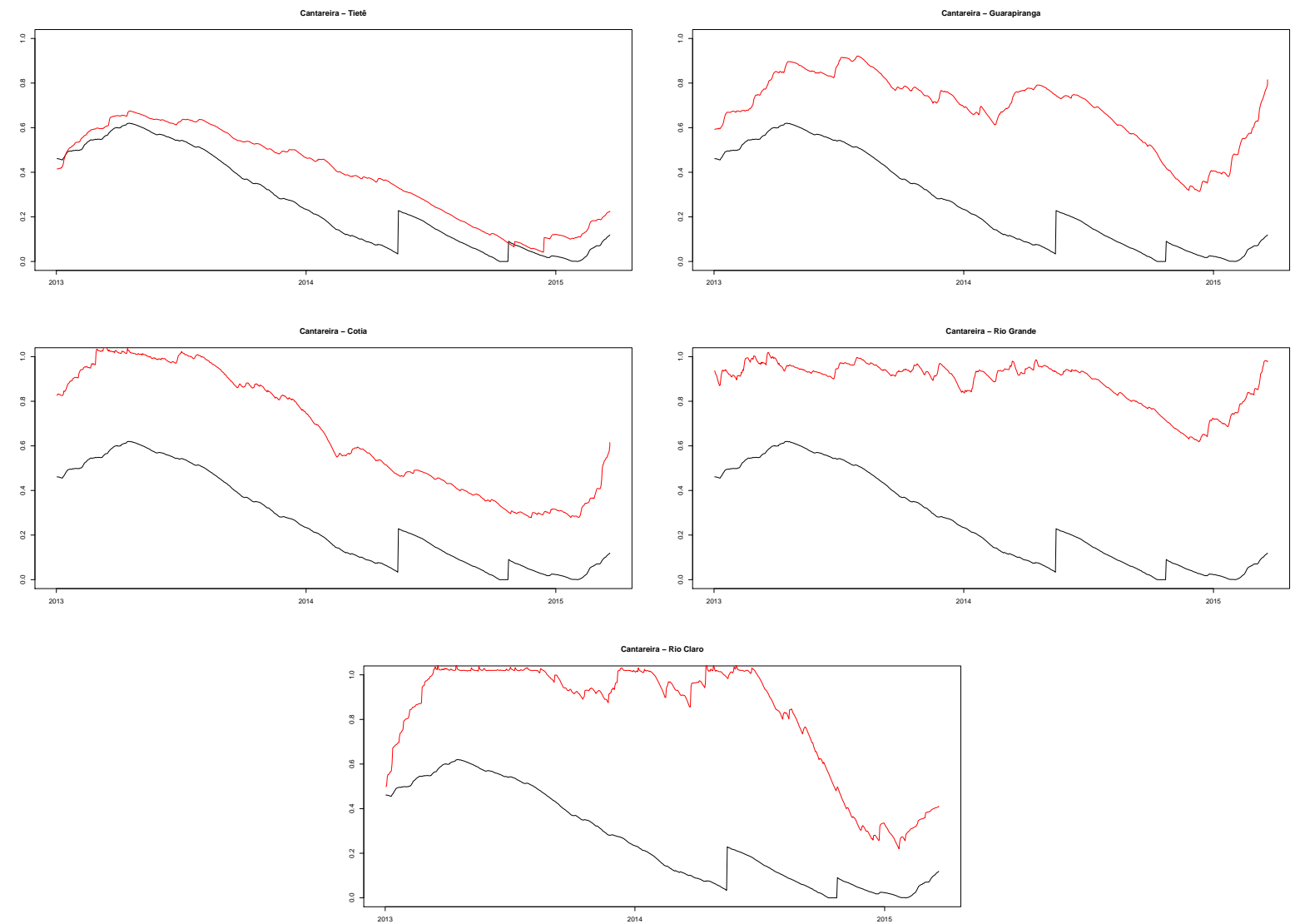

Figura 6.50: Gráfico da relação quadrática entre o sistema da cantareira com os demais sistemas a partir do ano de 2013. 

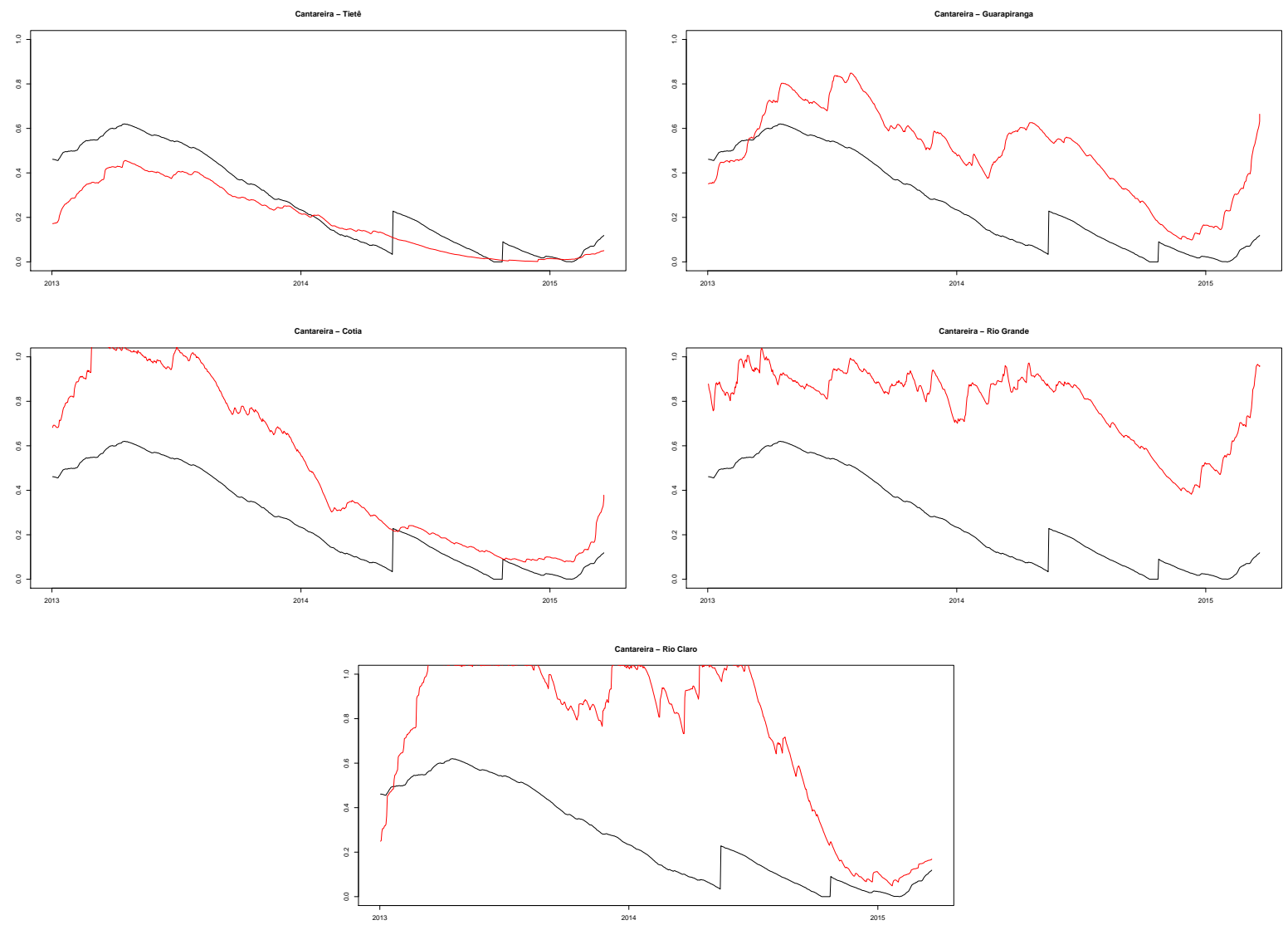

Figura 6.51: Gráfico da relação quadrática entre o sistema da cantareira com os demais sistemas a partir do ano de 2013.

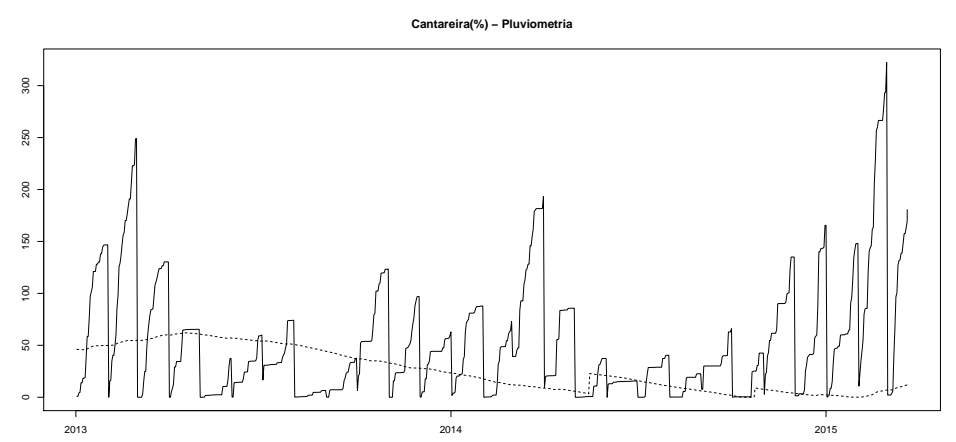

Figura 6.52: Gráfico da relação quadrática entre o sistema da cantareira com os demais sistemas a partir do ano de 2013. 
Consideramos o modelos especificado da seguinte forma:

$$
\begin{aligned}
& \eta_{t}=F^{\prime} \theta_{t} \\
& \theta_{t}=G \theta_{t-1}+\boldsymbol{\beta} \boldsymbol{x}_{t}+\omega, \quad \omega \sim N(0, W) .
\end{aligned}
$$

$\operatorname{com} G=J_{2}$.

Como covariáveis candidatas aos modelos, consideramos:

- $\boldsymbol{x}=$ Alto Tietê;

- $\boldsymbol{x}=\left(\right.$ Alto Tietê, Cotia $\left.^{2}\right)$

- $\boldsymbol{x}=\left(\right.$ Alto Tietê, Tietê, Cotia $\left.^{2}\right)$;

- Modelo sem covariável.

Onde todas as covariáveis foram aplicadas com defasagem de 1 dia. Vale destacar também que em 16 de maio e 24 de outubro de 2014 foi liberada uma cota do volume morto que alavancou o nível de água do sistema da Cantareia. Com isso, faz-se necessário o uso de intervenção externa na modelagem da série. Dentre os métodos de intervenção em modelos dinâmicos, o mais adequado para esta situação é a intervenção via ruído adicional de evolução nos instantes em que foram liberadas as cotas de volume morto (vide B; West e Harrison, 1997, Cap. 11, pag. 375). No período 16/05/2014 foi aplicado o ruído:

$$
\begin{gathered}
\boldsymbol{\theta}_{t}=\boldsymbol{G} \boldsymbol{\theta}_{t-1}+\boldsymbol{\omega}_{t}+\boldsymbol{\epsilon}_{t}, \quad \boldsymbol{\epsilon}_{t} \sim N\left(\boldsymbol{h}_{t}, \boldsymbol{H}_{t}\right) \\
\boldsymbol{h}_{t}=\left(\begin{array}{c}
2,17 \\
0 \\
0
\end{array}\right), \quad \boldsymbol{H}_{t}=\left(\begin{array}{ccc}
0,005 & 0 & 0 \\
0 & 0,01 & 0 \\
0 & 0 & 0,01
\end{array}\right) .
\end{gathered}
$$

No período 24/10/2014 foi aplicado o ruído:

$$
\begin{array}{cc}
\boldsymbol{\epsilon}_{t} \sim N\left(\boldsymbol{h}_{t}, \boldsymbol{H}_{t}\right) \\
\boldsymbol{h}_{t}=\left(\begin{array}{c}
3,74 \\
0 \\
0
\end{array}\right), \quad \boldsymbol{H}_{t}=\left(\begin{array}{ccc}
0,005 & 0 & 0 \\
0 & 0,01 & 0 \\
0 & 0 & 0,01
\end{array}\right) .
\end{array}
$$

Os valore de $\boldsymbol{h}_{t}$ foram escolhidos de acordo com os saltos observados na série nestes dois períodos e a variância $\boldsymbol{H}_{t}$ foi calibrada por meio de alguns ajustes.

Para a escolha das prioris de $W$ foi utilizada uma parte inicial da série (correspondente ao ano de 2013) para aprendizagem utilizando uma priori pouco informativa. Foram utilizadas as prioris: $W_{1: 2}^{-1} \sim W(10 / 2, \operatorname{diag}(90,100)), W_{3}^{-1} \sim G(6 / 2,0,005 \times 6 / 2)$. No modelo $B I Z U$ foi utilizada a priori para a probabilidade de zeros $\alpha \sim B(2,50)$ centrada na proporção de zeros observados na série. Para as covariáveis, quando utilizadas, foram utilizadas prioris $\beta \sim N(0,0,7)$. Para a priori de $\theta_{0}$ escolhida média $m_{0}=\left(m_{01} \times(0,9,0,1), m_{02}\right)^{\prime}$ onde $m_{01}=\operatorname{logit}\left(y_{1}\right)$ para ambos os modelos e $m_{02}=-\log (\sqrt{\operatorname{var}(y)})$ no modelo BIZU e $m_{02}=\log (\sqrt{\operatorname{var}(y)})$. A variância foi dada por $C_{0}=\operatorname{diag}(0,5,0,1,0,1)$. O número de partículas utilizadas foi $N=10000$. O tempo computacional do processo foi bem alto, durou cerca 4 horas, para este número de partículas.

Para verificar o melhor ajuste, utilizamos os critérios DIC e $L M P L$. Pela Tabela (6.1) podemos verificar que o modelo Tobit mais adequado foi o modelo com $\boldsymbol{x}=\left(\right.$ Alto Tietê, Cotia $\left.{ }^{2}\right)$. Pela Tabela (6.2) podemos verificar que o modelo BIZU sem covariáveis teve melhor desempenho pelo critério $D I C$, enquanto o modelo com $\boldsymbol{x}=\left(\right.$ Alto Tietê, $\mathrm{Cotia}^{2}$ ) apresentou melhor desempenho pelo critério $L M P L$. Cabe ressaltar que graficamente praticamente não foi observada diferença entre os modelos com as diferentes covariáveis, além disso, todos parâmetros $\beta$ foram estimados em valores negativos bem próximos de zero. 
Para nível de comparação entre os dois modelos, vamos utilizar os modelos com sua melhor especificação. A Tabela (6.3) apresenta a comparação entre o modelo Tobit, Bizu e também o modelo Beta, onde as observações iguais a zero foram substituídas por $\epsilon=10^{-4}$. Para a comparação foram utilizados os critérios DIC, LMPL e as métricas MSE, MAD, MAPE, MdAPE, SMAPE e $M A S E$, que estão descritas em (A). Nota-se que o modelo Tobit apresentou melhor desempenho pelos critério DIC e LMPL. O modelo Beta foi inferior a ambos os modelos nos critério de ajuste, no entanto apresentou melhor desempenho nas métricas, por se tratar de um modelo sem componente discreta.

Graficamente nota-se que os modelos apresentaram comportamentos bem semelhantes, entretanto quando avaliamos os gráficos de predição 30 passos a frente (vide Figuras (6.58), (6.65) e (6.67)), notamos que os modelos beta e $B I Z U$ apresentaram pior desempenho. Observa-se na Tabela (6.4) o critério $D I C$ e a medida $M S E$ construídos somente para os 30 passos a frente estimados sem as últimas 30 observações da série. Percebe-se claramente a superioridade do modelo Tobit na predição dos dados não observados.

No entanto, pela Figuras (6.64) nota-se que o modelo BIZU se adaptou melhor para a mudança de tendência no final da série, conseguindo captar melhor o crescimento do nível de água a partir de fevereiro de 2015. Além disso, o modelo BIZU se mostra interessante ao permitir a estimativa do risco de ocorrência do estado crítico por meio da estimativa do parâmetro de probabilidade de mistura $\alpha$ (vide Figura (6.60)). 
Tabela 6.1: Comparação entre as especificações do modelo Tobit segundo os critérios DIC e LMPL.

\begin{tabular}{l|c|c}
\hline Covariáveis & DIC & LMPL \\
\hline Alto Tietê & 16347,48 & $-4405,25$ \\
Alto Tietê + Cotia $^{2}$ & 13858,17 & $-4023,79$ \\
Alto Tietê + Cotia + Cotia $^{2}$ & 17976,90 & $-4405,25$ \\
- & 27634,57 & $-4288,90$ \\
\hline
\end{tabular}

Tabela 6.2: Comparação entre as especificações do modelo BIZU segundo os critérios DIC e LMPL.

\begin{tabular}{l|c|c}
\hline Covariáveis & DIC & LMPL \\
\hline Alto Tietê & 19340,63 & $-4826,46$ \\
Alto Tietê + Cotia $^{2}$ & 12805,45 & $-4364,27$ \\
Alto Tietê + Cotia + Cotia $^{2}$ & 16382,63 & $-4526,94$ \\
- & 10614,22 & $-4410,46$ \\
\hline
\end{tabular}

Tabela 6.3: Comparação entre os modelos Tobit, BIZU e Beta pelos critédios DIC, LMPL e métricas de predição.

\begin{tabular}{l||c|c||c|c|c|c|c|c}
\hline Modelo & DIC & LMPL & MSE & MAD & MAPE & MdAPE & SMAPE & MASE \\
\hline Tobit & 13858,17 & $-4023,79$ & 0,00011 & 0,0028 & 0,1049 & 0,0004 & 0,0873 & 1,4871 \\
BIZU & 10614,22 & $-4410,46$ & 0,00012 & 0,0029 & 0,1024 & 0,0004 & 0,0997 & 1,4929 \\
Beta & 13686,16 & $-4320,63$ & 0,00009 & 0,0025 & 0,0057 & 0,0004 & 0,15 & 01,3440 \\
\hline
\end{tabular}

Tabela 6.4: Comparação entre os modelos Tobit, BIZU e Beta pelo critédio DIC e MSE somente na previsão de 30 passos a frente.

\begin{tabular}{l||c|c}
\hline Modelo & $D I C_{p}$ & $M S E_{p}$ \\
\hline Tobit & $-1684,3$ & 0,0037 \\
BIZU & 2218,8 & 0,0060 \\
Beta & 2570,7 & 0,0070 \\
\hline
\end{tabular}



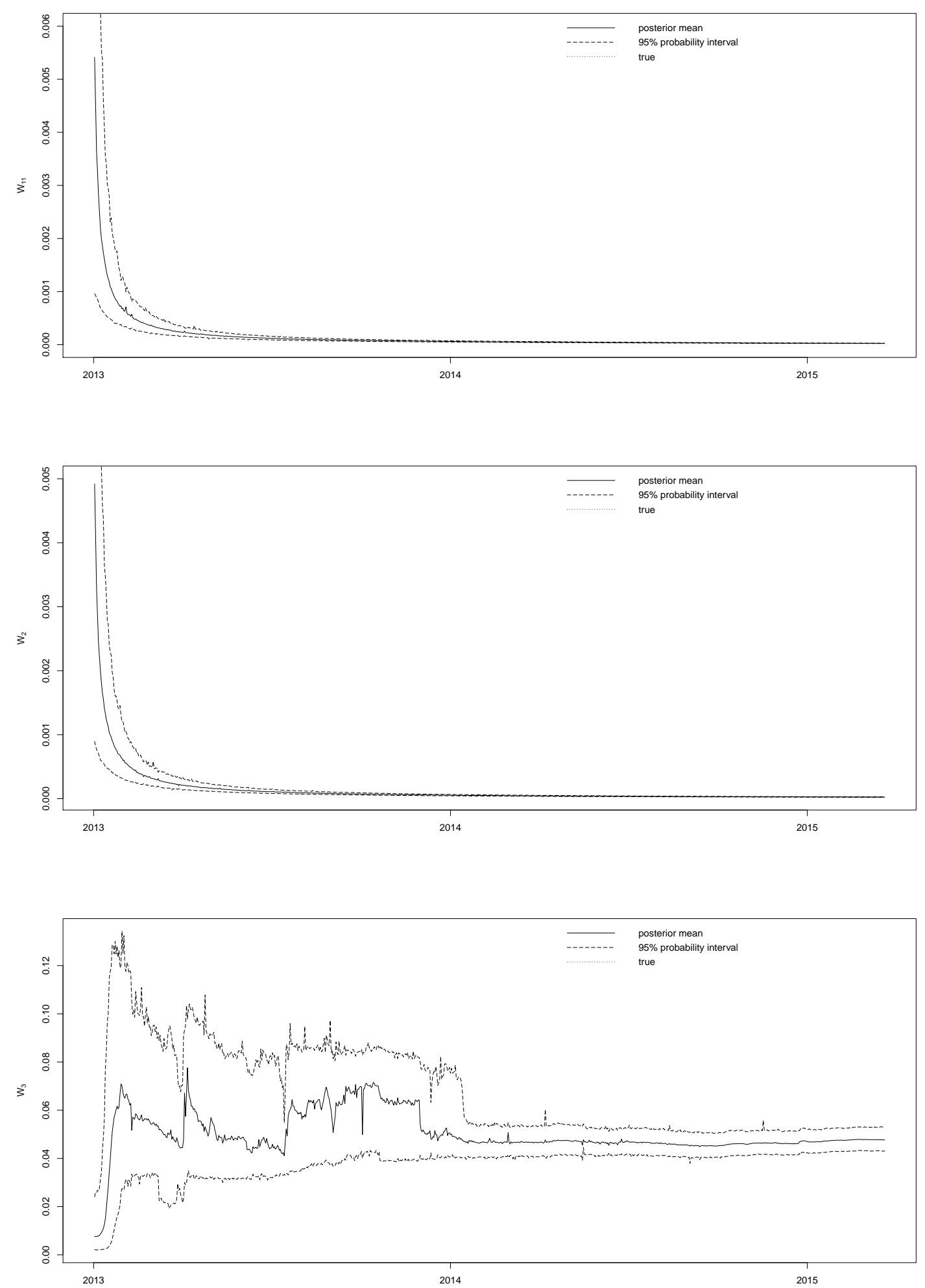

Figura 6.53: Gráfico da estimativa das componentes da variância $W$ do modelo dinâmico tobit. 

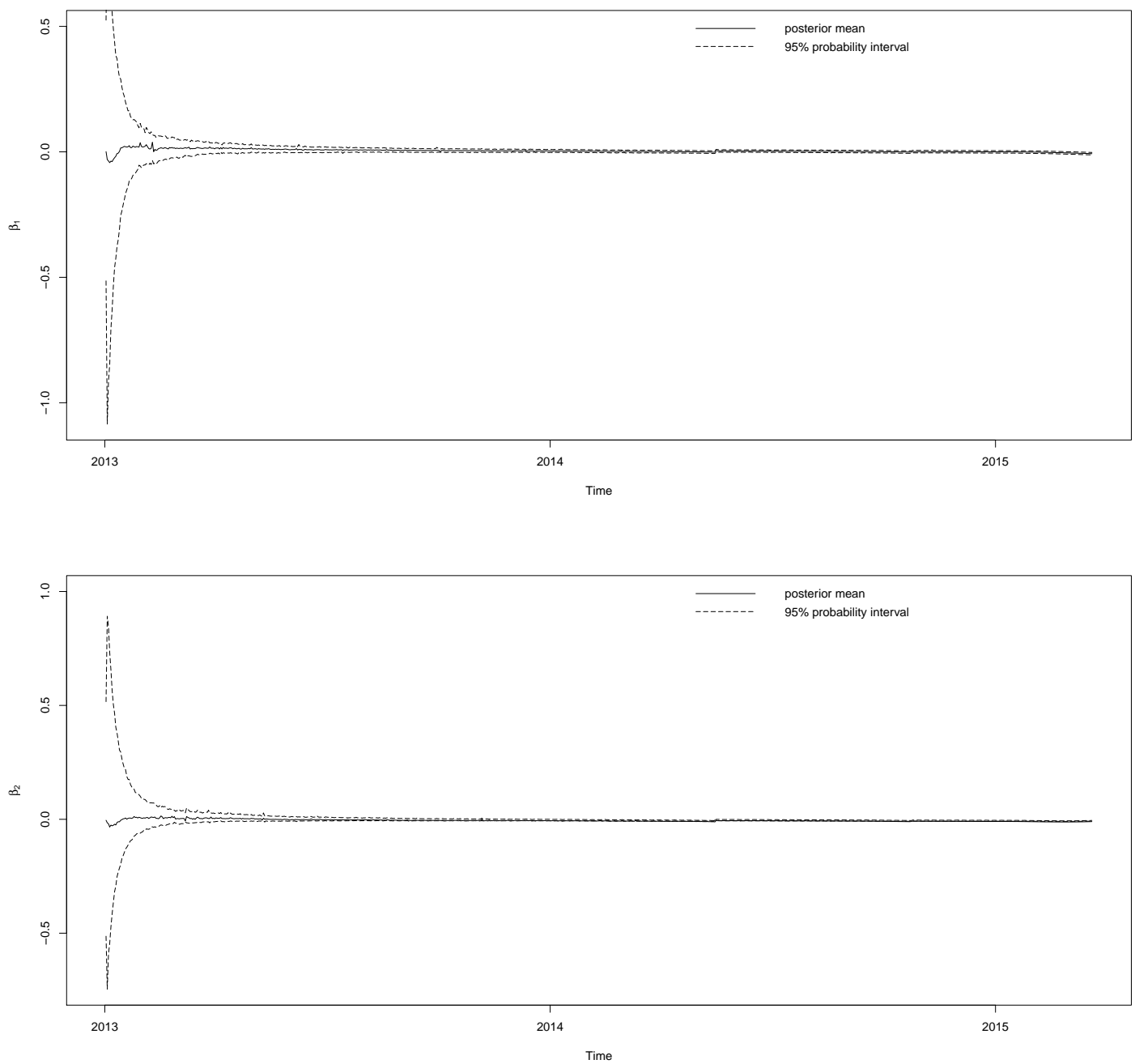

Figura 6.54: Gráfico da estimativa dos parâmetros $\beta$ do modelo dinâmico tobit. 

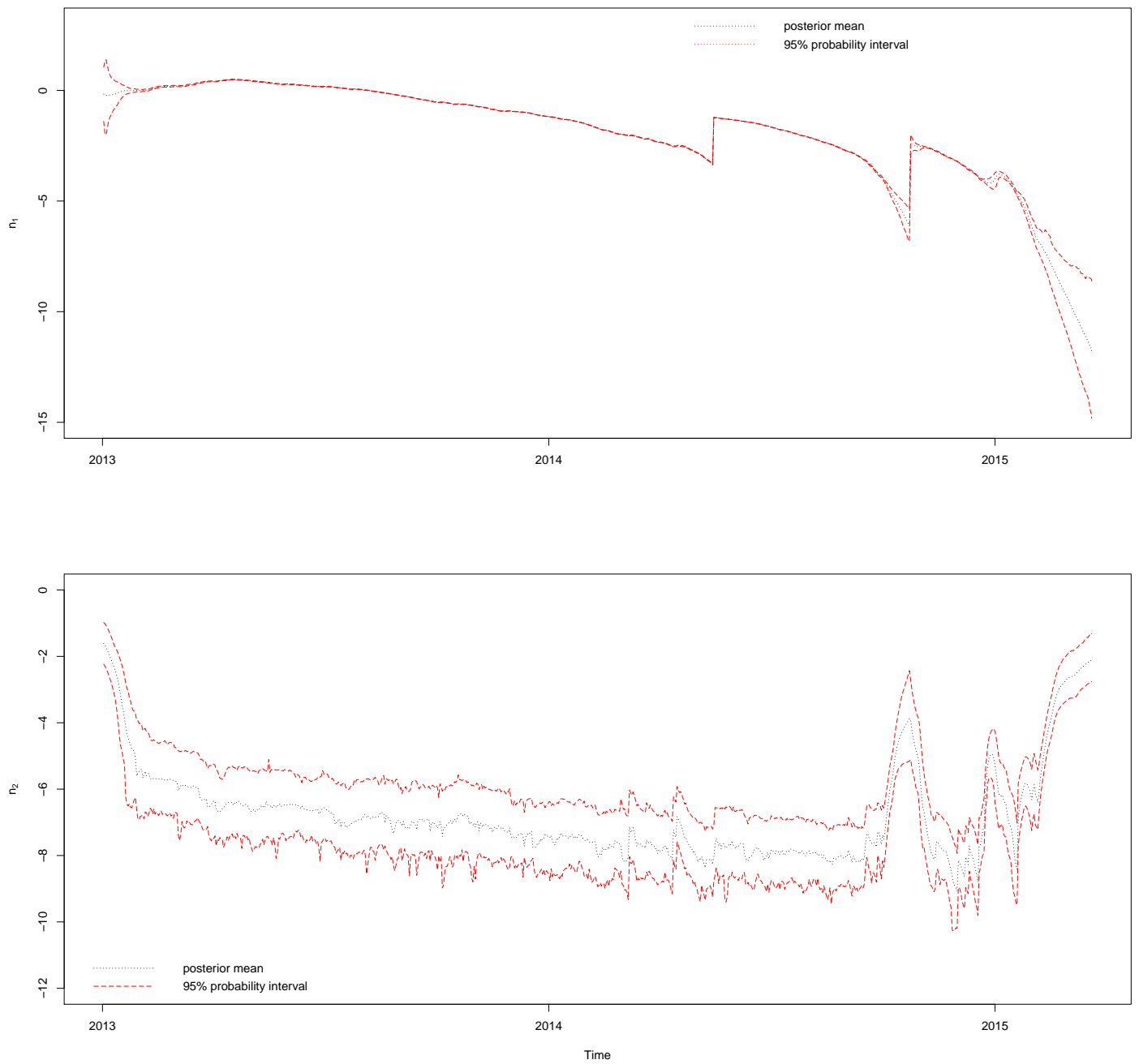

Figura 6.55: Gráfico dos preditores lineares $\eta$ estimados pelo modelo dinâmico tobit. 

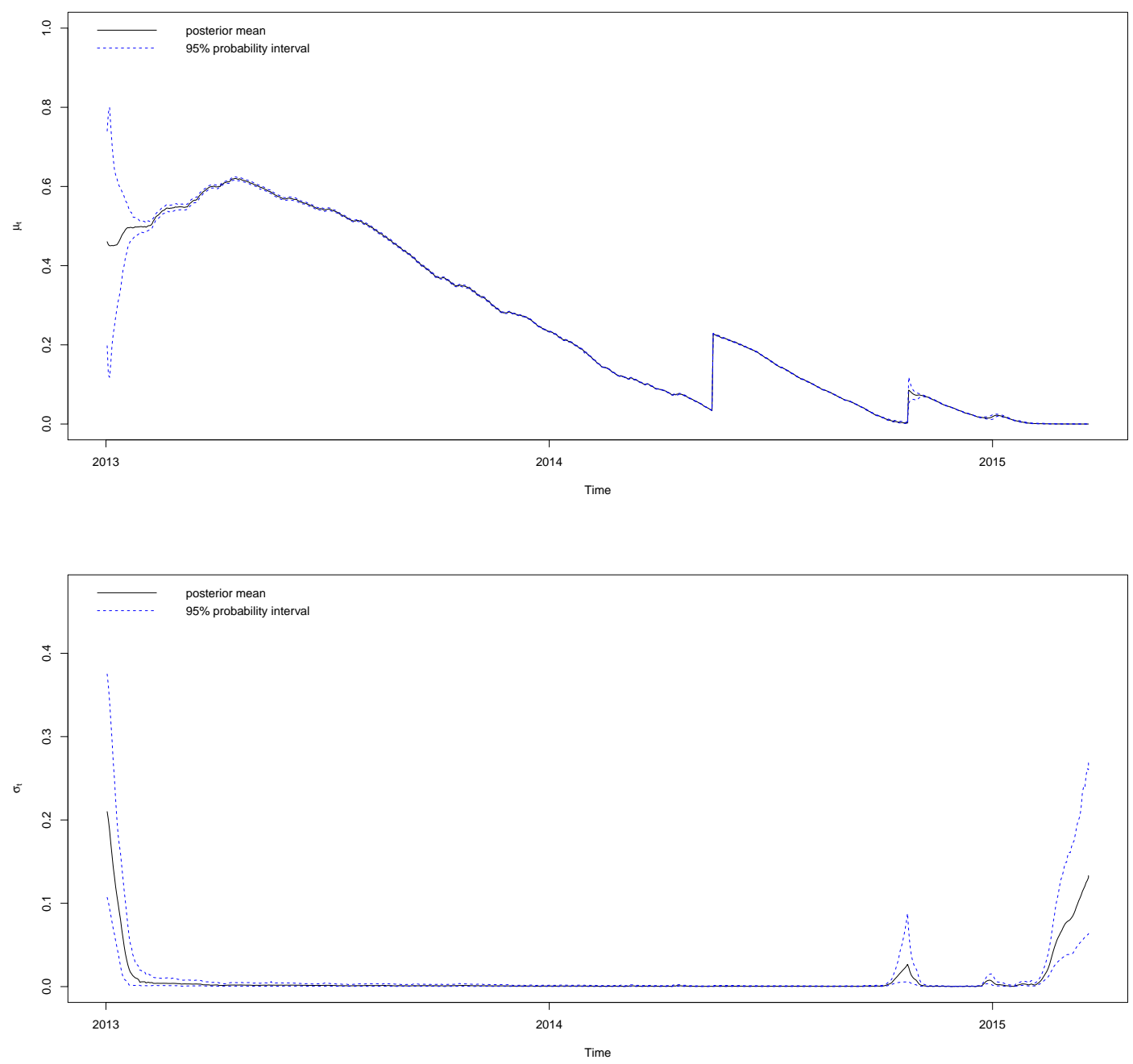

Figura 6.56: Gráfico dos estados $\mu_{t}$ e $\sigma_{t}$ do modelo dinâmico tobit.

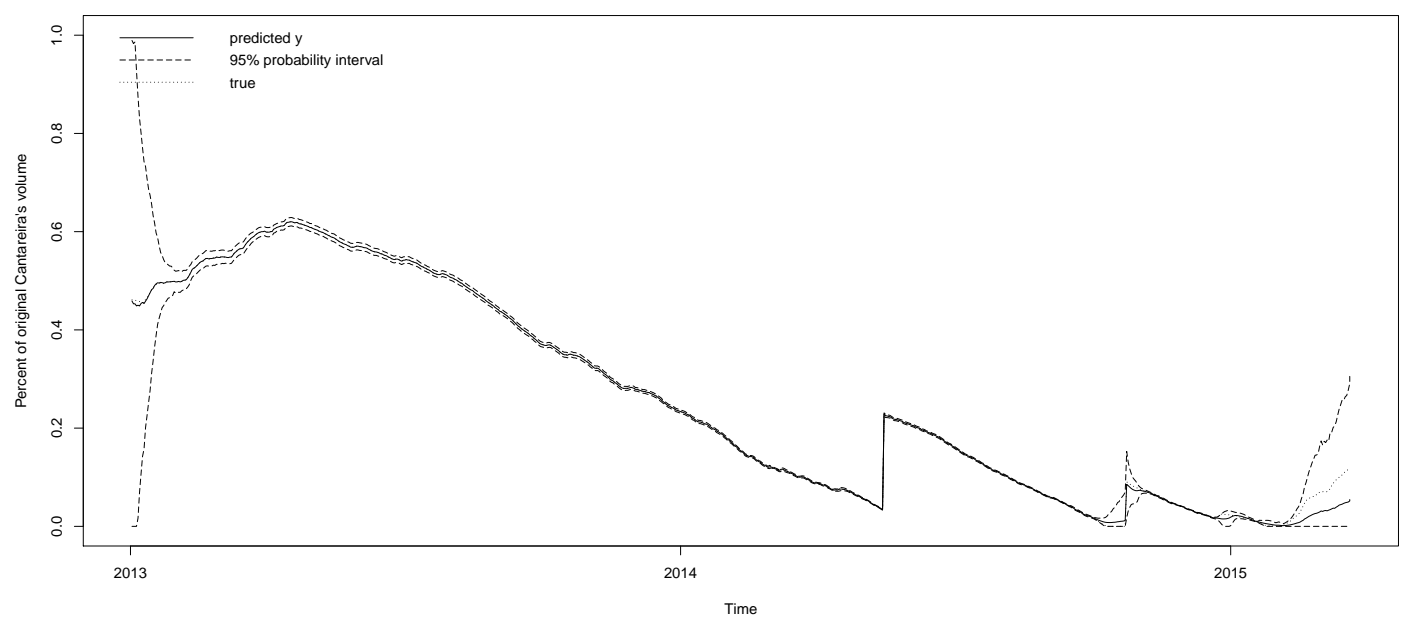

Figura 6.57: Grafico da distribuição preditiva $p\left(y_{t} \mid D_{t-1}\right)$ do modelo dinâmico tobit. 


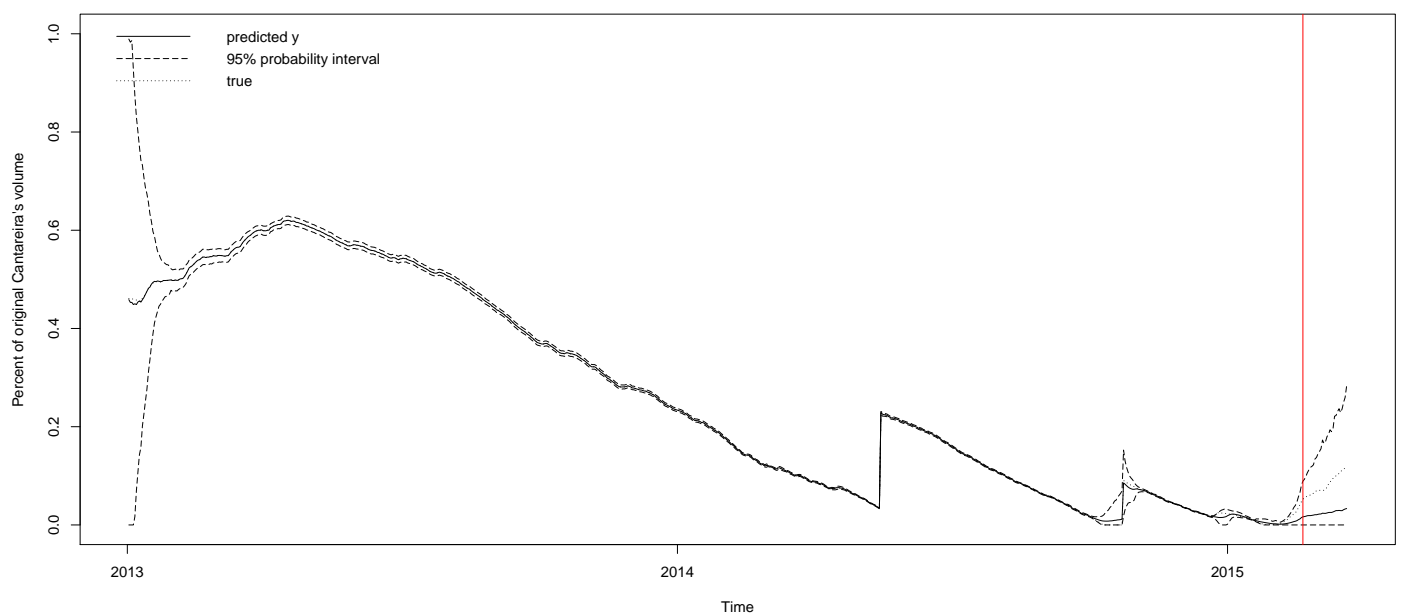

Figura 6.58: Grafico da distribuição preditiva $p\left(y_{t} \mid D_{t-1}\right)$ com predição de 30 passos a frente do modelo dinâmico tobit. 

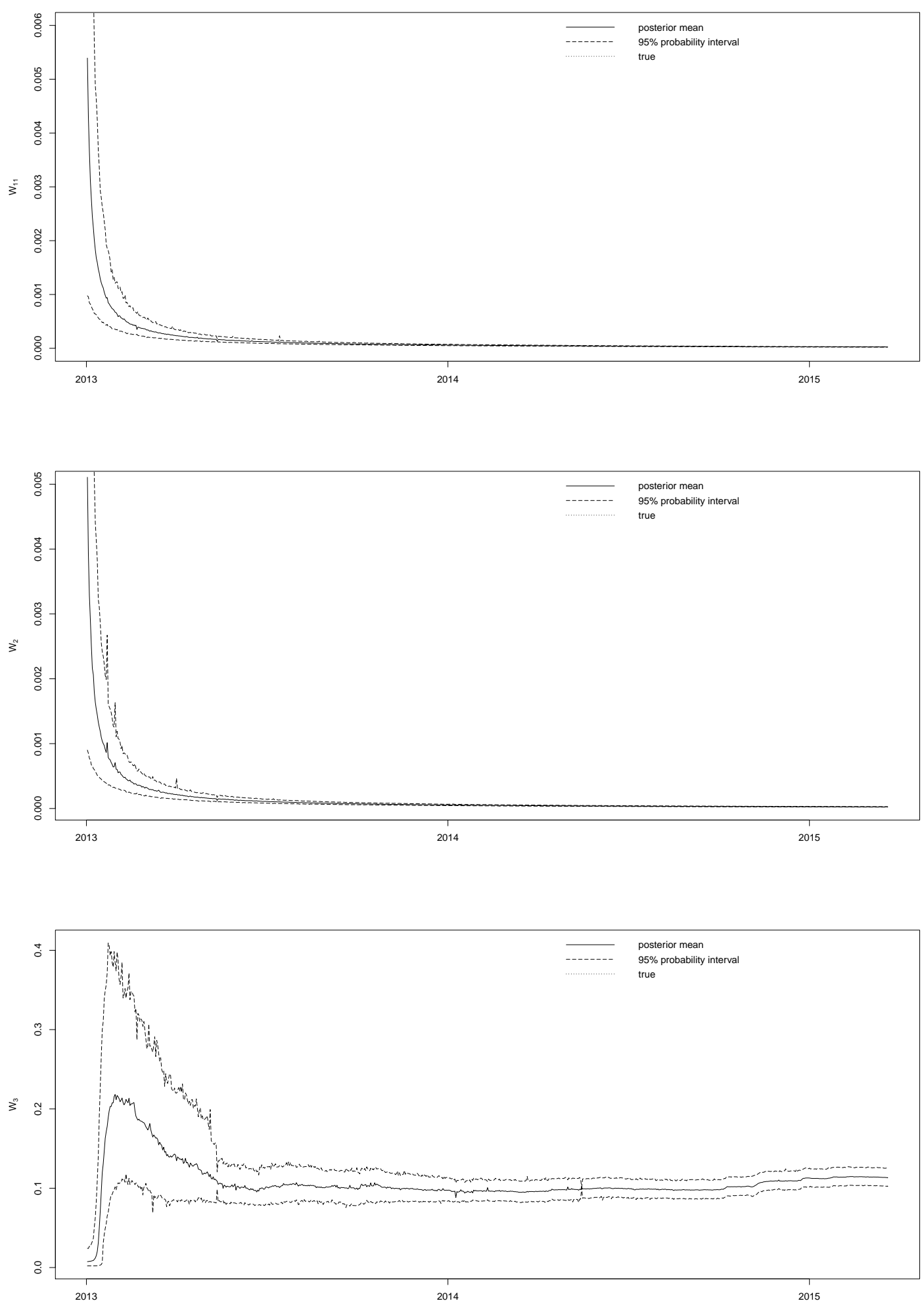

Figura 6.59: Gráfico da estimativa das componentes da variância $W$ do modelo dinâmico BIZU. 


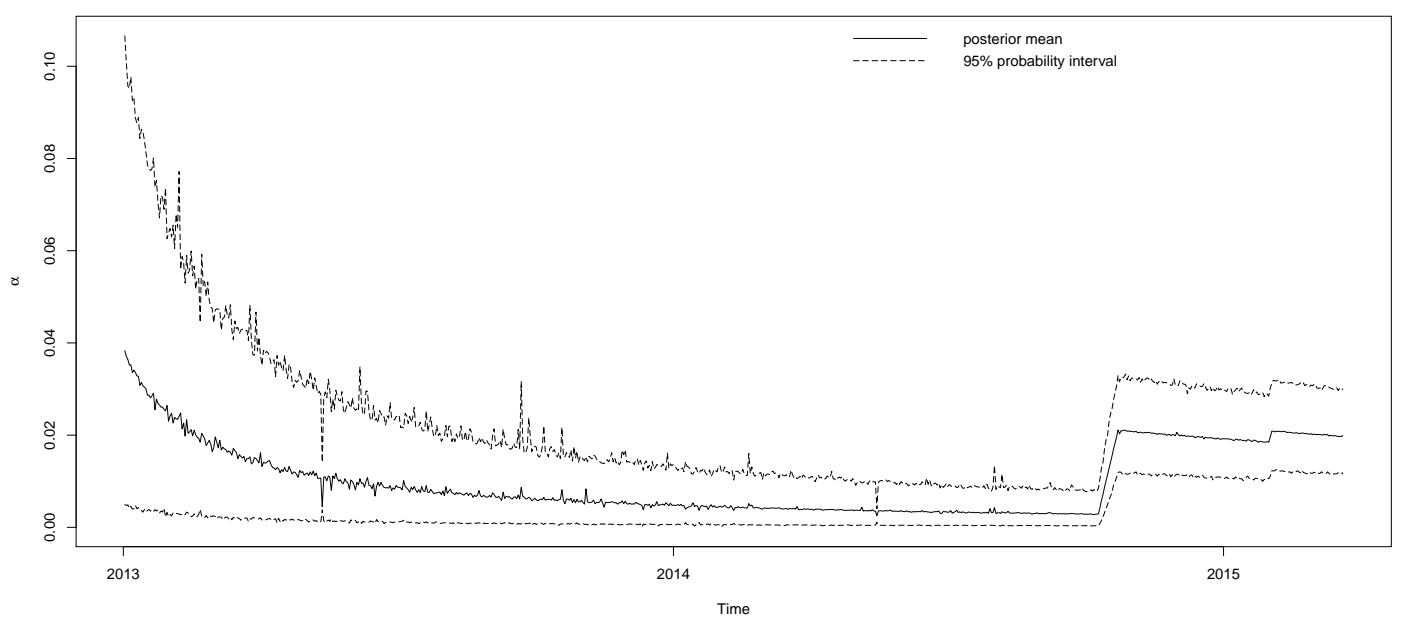

Figura 6.60: Gráfico da estimativa da componente discreta $\alpha$ do modelo dinâmico BIZU.
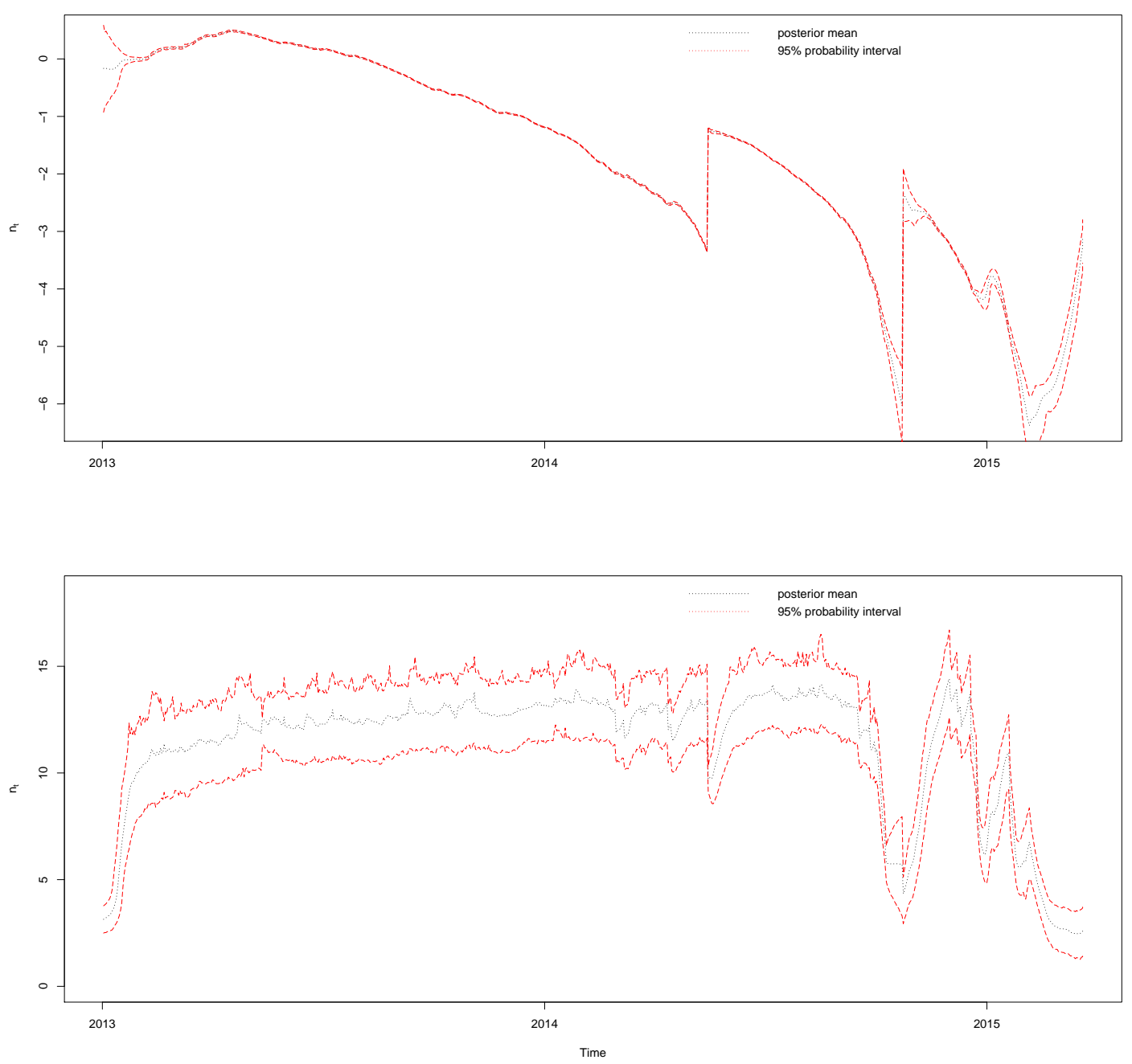

Figura 6.61: Gráfico dos preditores lineares $\eta$ estimados pelo modelo dinâmico BIZU. 

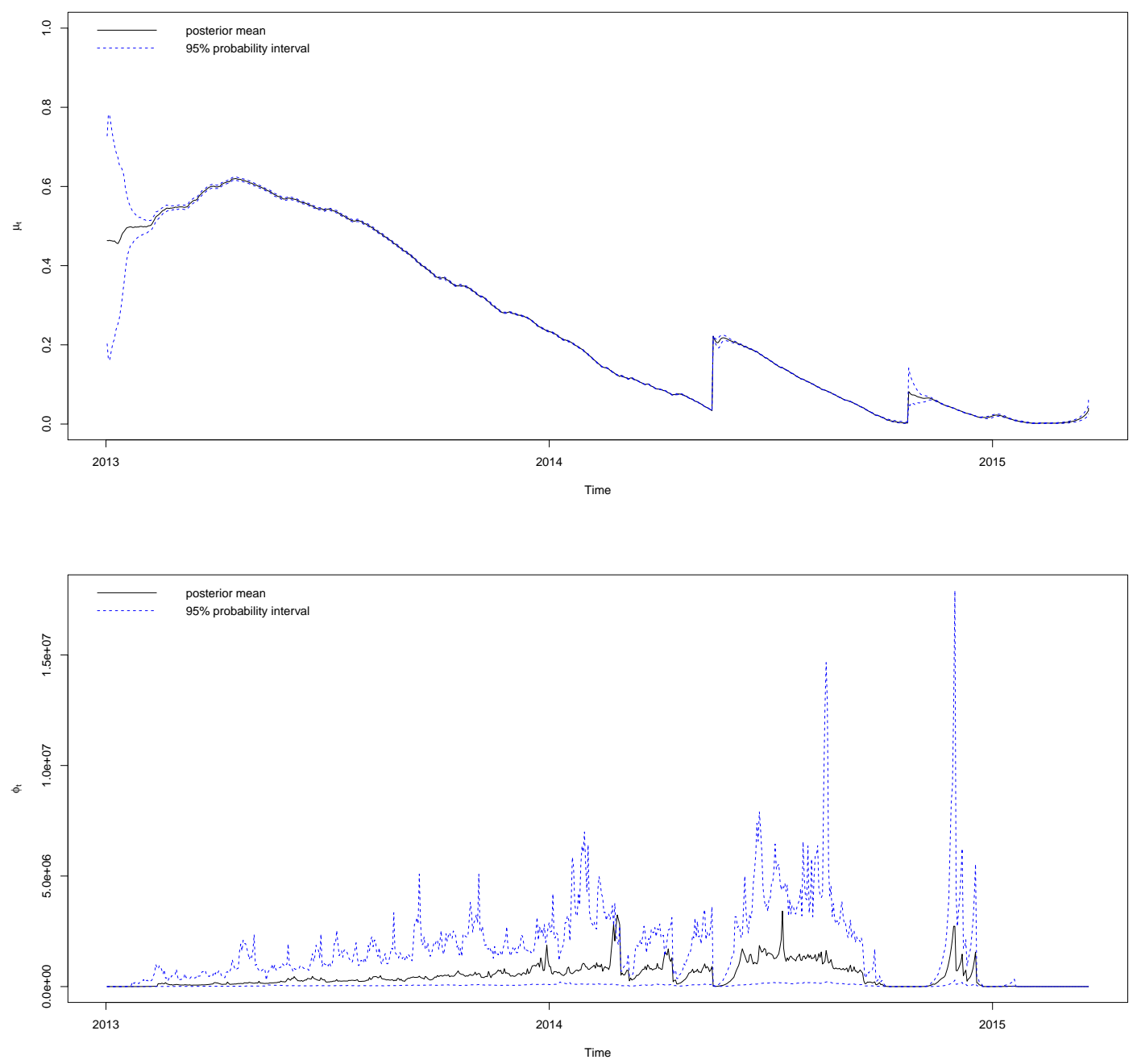

Figura 6.62: Gráfico dos estados $\mu_{t}$ e $\phi_{t}$ do modelo dinâmico BIZU.

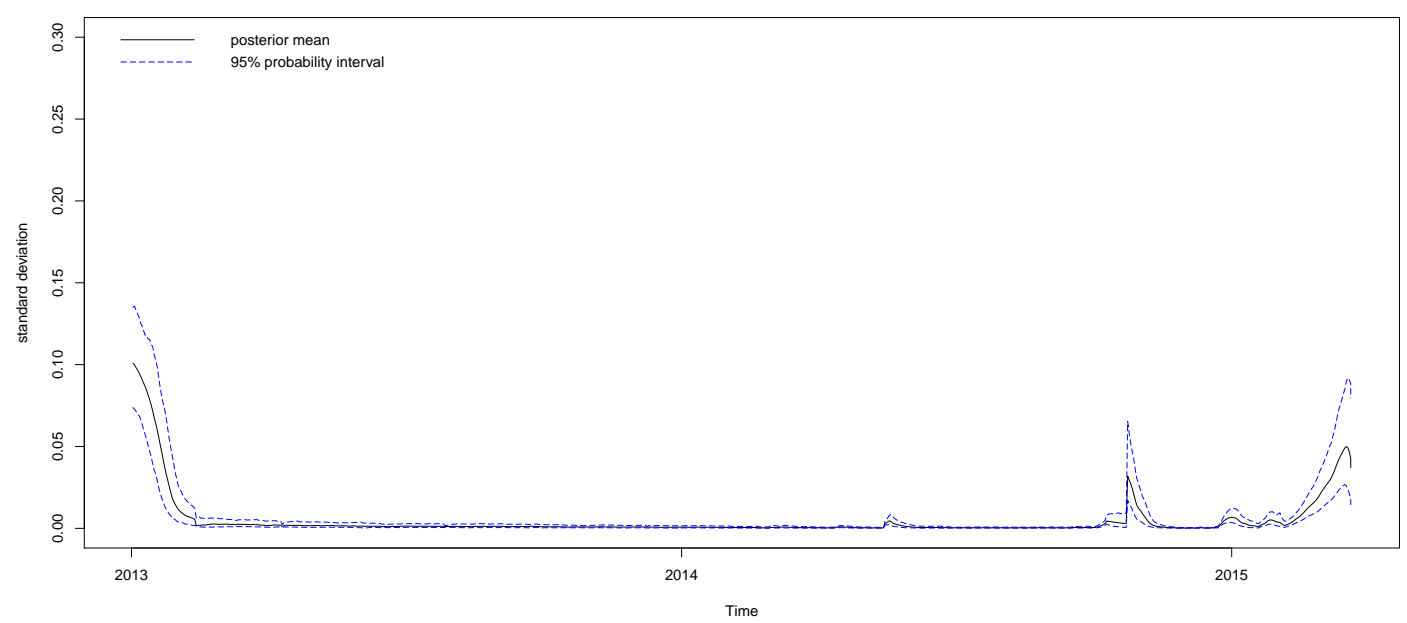

Figura 6.63: Gráfico do desvio padrão da componente contínua do modelo dinâmico BIZU. 


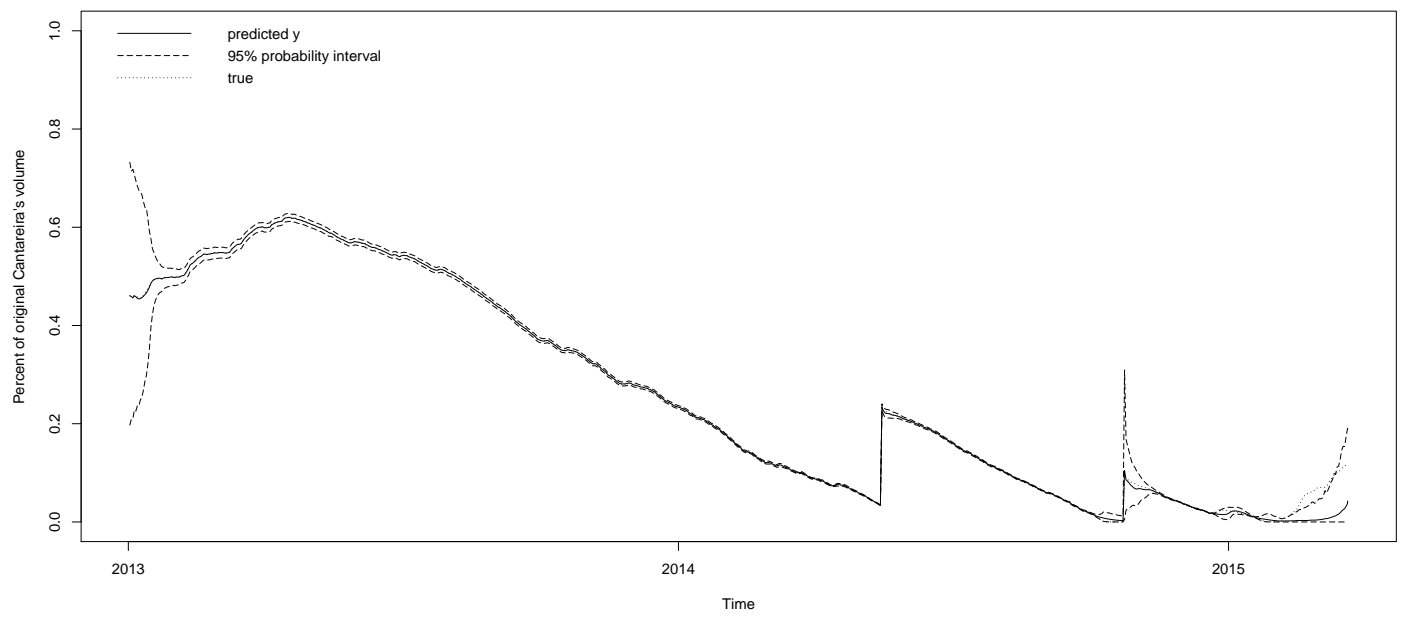

Figura 6.64: Gráfico da distribuiçãa preditiva $p\left(y_{t} \mid D_{t-1}\right)$ da componente contínua do modelo dinâmico $B I Z U$.

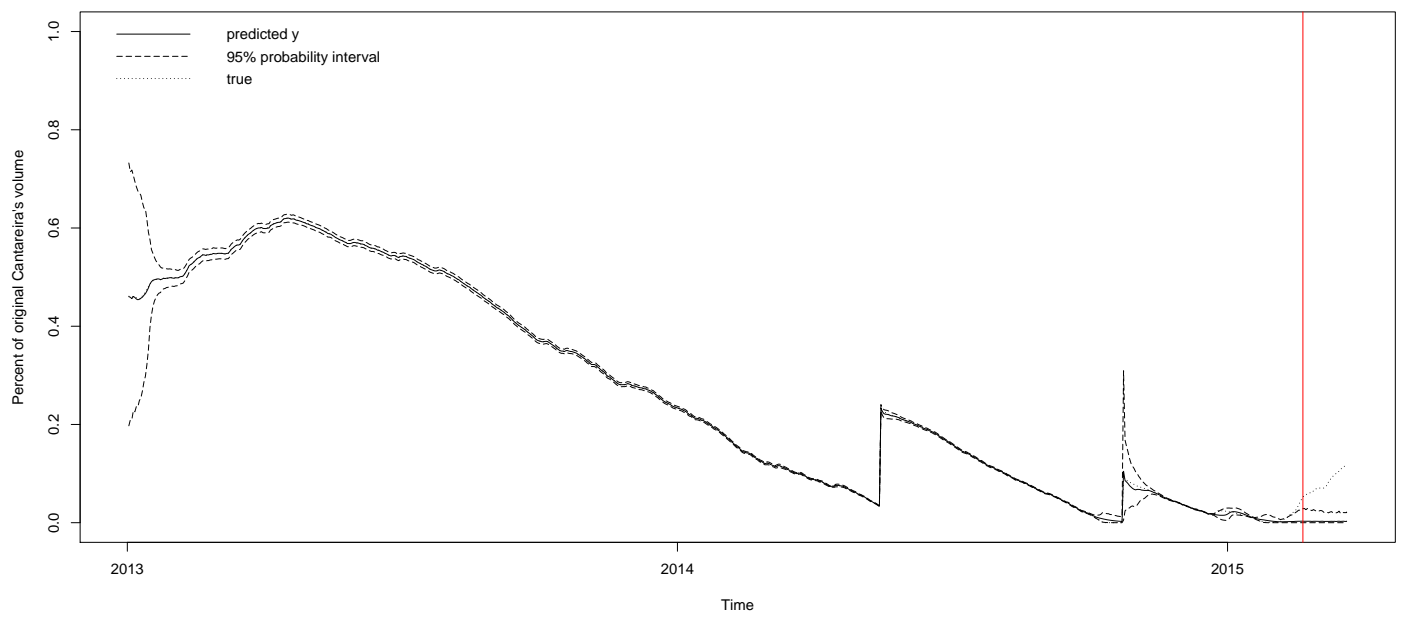

Figura 6.65: Gráfico da distribuição preditiva $p\left(y_{t} \mid D_{t-1}\right)$ com predição de 30 passos a frente da componente contínua do modelo dinâmico BIZU.

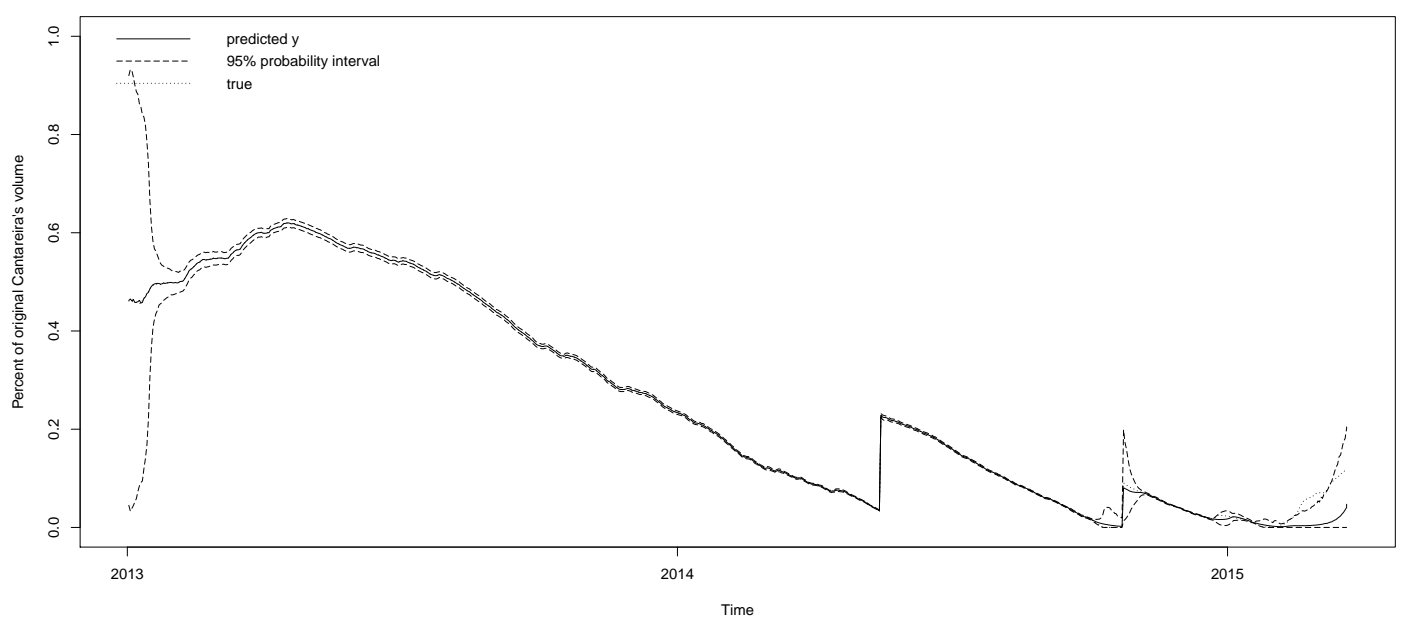

Figura 6.66: Gráfico da distribuição preditiva $p\left(y_{t} \mid D_{t-1}\right)$ do modelo dinâmico beta. 


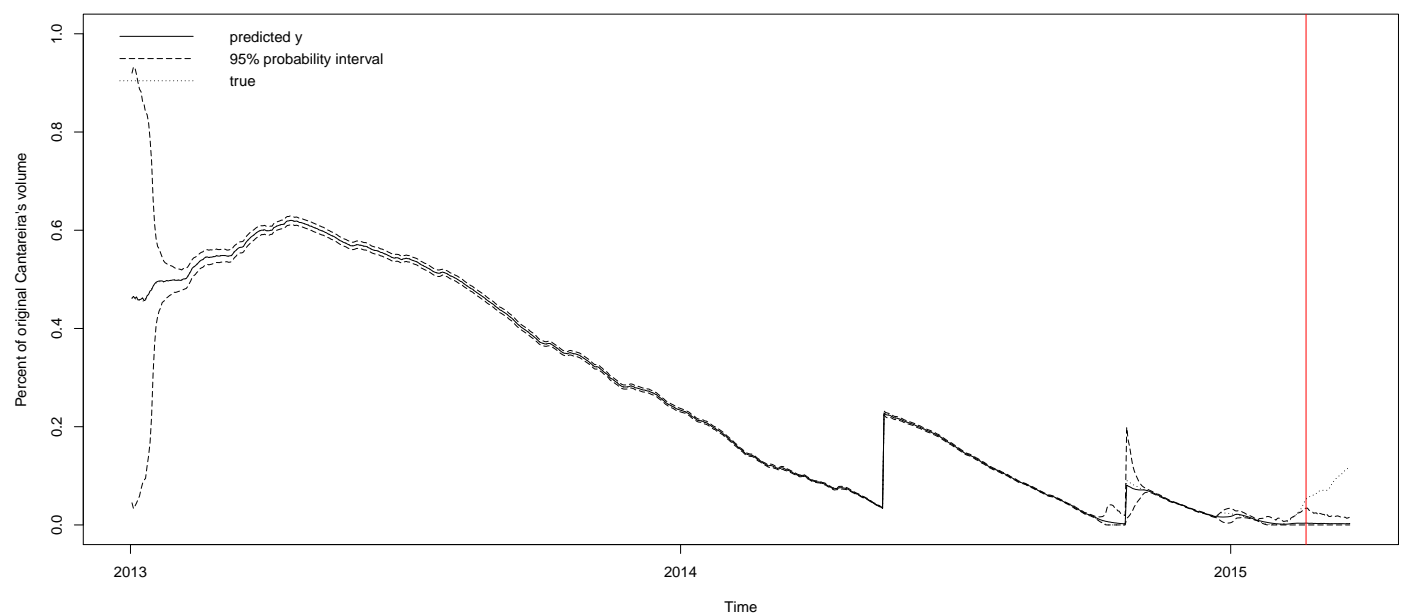

Figura 6.67: Gráfico da distribuição preditiva $p\left(y_{t} \mid D_{t-1}\right)$ com predição de 30 passos a frente do modelo dinâmico beta. 


\section{Capítulo 7}

\section{Conclusões}

Existem diversos contextos práticos em que nos deparamos com a necessidade de analisar dados restritos a um intervalo limitado, dados restrito no intervalo $[0,1]$ são os casos mais comuns encontrado no cotidiano como variáveis que representam medidas de proporção ou taxa. Os modelos clássicos de regressão e de séries de tempo mais difundidos na literatura não são os mais adequados para lidar com o problema.

Este trabalho explorou por meio da inferência Bayesiana como lidar com dados de proporção no seu caso mais geral, quando há presença de valores extremos, zero e/ou um em análises estática e de série de tempo.

\subsection{Considerações Finais}

Podemos separar os modelos estudados em duas classes: os modelos Beta inflacionados de zeros e uns e a classe de modelos Tobit. Observamos que ambos os modelos possuem desempenho satisfatórios para a estrutura de dados em questão. Contudo, identificamos certas circunstâncias em que um modelo é mais adequado que o outro para a análise.

Pela flexibilidade da distribuição Beta, o modelo Beta inflacionados de zeros e uns é mais adequados para dados com comportamento que foge da simetria. No entanto, notamos que a distribuição Beta é bastante sensível a valores próximos das extremidades do intervalo, o que prejudica a avaliação da função da verossimilhança e consequentemente as distribuições a posteriori dos parâmetros nos modelos Beta inflacionados de zero e uns (BIZU). O modelo Tobit apresenta a vantagem de tratabilidade permitida pela distribuição normal, além de não apresentar problemas de ajuste na presença de valores em uma vizinhança muito próxima da extremidade.

O uso da inflação dos valores extremos possui a grande vantagem de permitir uma atenção especial a estas observações, de modo que é possível ter uma interpretação mais detalhada de sua ocorrência, esta abordagem é bastante interessante em estudos em que deseja-se avaliar o riscos, como no caso da série de nível de água do sistema da cantareira apresentado em 6.3 ou no caso de proporção de fatura do cartão de uma instituição financeira, onde há grande interesse em estudar o risco de inadimplência. Em outras circunstâncias os valores extremos são interpretados como censura nos dados, neste caso o modelo Tobit é mais indicado. Além disso o modelo Tobit ainda permite extensões para se adequar a inflações e dados assimétricos como discutidos no Capítulo 2.

Por fim, vale ressaltar a importância do uso de filtro de partículas no contexto dos modelos dinâmicos Bayesianos em casos de mistura de distribuições e distribuições censuradas, onde não há soluções analíticas para a obtenção das distribuições a posteriori e técnicas de MCMC ainda deixam a desejar pelo custo computacional e a perda da análise sequencial em uma série temporal.

\subsection{Sugestões para Pesquisas Futuras}

No contexto dos modelos Tobit há diversas extensões que podem ser exploradas para análises de regressão Bayesianas. Dentre elas podemos citar modelos "skew" e "log-skew" (Chai e Baikey, 
2008) e modelos bimodais e bimodais assimétricos (Gómez et al., 2009 e de Martinez et al., 2012).

Para estudos de modelos de espaços de estados, pode-se estudar a necessidade da implementação de modelos dinâmicos Tobit inflacionados e Tobit assimétricos assim como apresentados no análise de regressão (Capítulo 3).

Como extensão ao modelo BIZU, Pereira et al. (2012) apresentou o modelo de regressão beta inflacionado e truncado que pode ser inserido no contexto da inferência Bayesiana e de modelos dinâmicos.

Outro tópico interessante seria combinar esta metodologia de modelos dinâmicos com modelos com mudança de regime, por meio da metodologia apresentada por McCormick et al. (2012). 


\section{Apêndice A}

\section{Métricas}

Considerando $y_{t}$ o dado observado no tempo $t$ e $\hat{y}_{t}$ o valor predito por $E\left(y_{t} \mid D_{t-1}\right), t=1, \ldots, T$. Temos as seguintes métricas para análise de qualidade da predição:

- MSE (Erro quadrático médio):

$$
\frac{\sum_{t=1}^{T}\left(y_{t}-\hat{y}_{t}\right)^{2}}{T}
$$

- MAD (Erro absoluto médio):

$$
\frac{\sum_{t=1}^{T}\left|y_{t}-\hat{y}_{t}\right|}{T}
$$

- MAPE (Erro percentual absoluto médio):

$$
\frac{1}{T} \sum_{t=1}^{T}\left|\frac{y_{t}-\hat{y}_{t}}{y_{t}}\right|
$$

- MdAPE (Erro percentual absoluto mediano):

$$
M d\left[\left|\frac{y_{t}-\hat{y}_{t}}{y_{t}}\right|\right]
$$

- SMAPE (Erro percentual simétrico absoluto mediano):

$$
\frac{1}{T} \sum_{t=1}^{T} \frac{\left|y_{t}-\hat{y}_{t}\right|}{\left(\left|y_{t}\right|+\left|\hat{y}_{t}\right|\right) / 2}
$$

- MASE (Erro escalonado absoluto mediano):

$$
\frac{1}{T} \sum_{t=1}^{T}\left(\frac{\left|e_{t}\right|}{\frac{1}{n-1} \sum_{t=2}^{T}\left|y_{t}-y_{t-1}\right|}\right),
$$

$\operatorname{com} e_{t}=y_{t}-\hat{y}_{t}$ 
APÊNDICE A 


\section{Apêndice B}

\section{Intervenção externa}

Segue algumas formas de intervenção externa em modelos dinâmicos.

\section{B.1 Ignorar observação $Y_{t}$}

A forma mais simples de intervenção é tratar o período em que ocorre algum fenômeno externo como um outlier e simplesmente descartar a observação $Y_{t}$. Desta forma, temos

$$
I_{t}=\left\{Y_{t} \text { dado faltante }\right\}
$$

de modo que $D_{t}=\left\{I_{t}, D_{t-1}\right\}$ que é efetivamente o mesmo que $D_{t-1}$.

\section{B.2 Ruído de evolução adicional}

Uma solução para contornar mudanças de condições que potencialmente afetem o decorrer da série é aumentando a sua incerteza sobre parte dos parâmetros do modelo. De forma geral, o modelo é aberto a intervenções em componentes do espaço de estados $\boldsymbol{\theta}_{t}$ de modo que cresça a incerteza simplesmente adicionando um novo termo de erro de evolução. Em muitos caso é desejado ainda incluir neste termo um deslocamento da média. Temos, portanto:

$$
I_{t}=\left\{\boldsymbol{h}_{t}, \boldsymbol{H}_{t}\right\},
$$

onde $\boldsymbol{h}_{t}$ corresponde ao vetor de média de $\boldsymbol{H}_{t}$ corresponde a matriz de covariância do componente de erro aleatório $\boldsymbol{\epsilon}_{t}$, com

$$
\boldsymbol{\epsilon}_{t} \sim N\left[\boldsymbol{h}_{t}, \boldsymbol{H}_{t}\right]
$$

\section{B.3 Intervenção subjetiva arbitrária}

O caso mais geral de intervenção em um modelo existente é simplesmente modificar a distribuição a priori de $\boldsymbol{\theta}_{t}$ para valores antecipando a mudança na série.

$$
I_{t}=\left\{\boldsymbol{a}_{t}^{*}, \boldsymbol{R}_{t}^{*}\right\},
$$

onde os valores $\boldsymbol{a}_{t}^{*}$ e $\boldsymbol{R}_{t}^{*}$ são atribuídos pelo pesquisador. Note que este caso é equivalente a intervenção por ruído adicional quando $\boldsymbol{R}_{t}^{*}=0$ e que implica em $\boldsymbol{\theta}_{t}=\boldsymbol{a}_{t}^{*}$ em probabilidade. No entanto,

$$
\left(\boldsymbol{\theta}_{t} \mid I_{t}, D_{t-1}\right) \sim N\left[\boldsymbol{a}_{t}^{*}, \boldsymbol{R}_{t}^{*}\right]
$$


não é mais consistente com a evolução do modelo. Para isso, temos que definir: $\boldsymbol{K}_{t}$ e $\boldsymbol{h}_{t}$ tais que

$$
\begin{aligned}
\boldsymbol{K}_{t} & =\boldsymbol{K}_{t} \boldsymbol{Z}_{t}^{-1}, \\
\boldsymbol{h}_{t} & =\boldsymbol{a}_{t}^{*}-\boldsymbol{K}_{t} \boldsymbol{a}_{t},
\end{aligned}
$$

$\operatorname{com} \boldsymbol{R}_{t}=\boldsymbol{Z}_{t} \boldsymbol{Z}_{t}^{\prime}$ e $\boldsymbol{R}_{t}^{*}=\boldsymbol{U}_{t} \boldsymbol{U}_{t}^{\prime}$. Com isso, temos:

$$
\boldsymbol{\theta}_{t}^{*}=\boldsymbol{G}_{t}^{*} \boldsymbol{\theta}_{t-1}+\boldsymbol{w}_{t}^{*}, \quad \boldsymbol{w}_{t}^{*} \sim N\left[\boldsymbol{h}_{t}^{*}, \boldsymbol{W}_{t}^{*}\right],
$$

com dado $D_{t-1}$ e $I_{t}, \boldsymbol{w}_{t}^{*}$ não é correlacionado com $\boldsymbol{\theta}_{t-1} \mathrm{e}$

$$
\begin{aligned}
\boldsymbol{G}_{t}^{*} & =\boldsymbol{K}_{t} \boldsymbol{G}_{t}, \\
\boldsymbol{w}_{t}^{*} & =\boldsymbol{a}_{t}^{*}-\boldsymbol{K}_{t} \boldsymbol{a}_{t}, \\
\boldsymbol{W}_{t}^{*} & =\boldsymbol{K}_{t} \boldsymbol{W}_{t} \boldsymbol{K}_{t}^{\prime} .
\end{aligned}
$$

\section{B.4 Inclusão de efeito de intervenção}

Em muitos casos é de interesse isolar os efeitos de uma intervenção, alocando um novo parâmetro para que define a mudança no modelo. Considere o exemplo:

$$
\boldsymbol{\theta}_{t}^{*}=\left(\mu_{t}^{*}, \beta_{t}, \gamma_{t}\right)
$$

O modelo dinâmico no tempo $t$ é sujeito a uma evolução adicional

$$
\left[\begin{array}{c}
\mu_{t}^{*} \\
\beta_{t} \\
\gamma_{t}
\end{array}\right]=\left[\begin{array}{ll}
1 & 0 \\
0 & 1 \\
0 & 0
\end{array}\right]\left[\begin{array}{l}
\mu_{t} \\
\beta_{t}
\end{array}\right]+\left[\begin{array}{l}
1 \\
0 \\
1
\end{array}\right] \gamma_{t},
$$

com distribuição atribuída a $\gamma_{t}$ determinando o valor esperado da mudança de nível.

$$
\boldsymbol{\theta}_{t}^{*}=\boldsymbol{K}_{t} \boldsymbol{\theta}_{t}+\boldsymbol{\epsilon}_{t},
$$

com

$$
\boldsymbol{K}_{t}=\left[\begin{array}{ll}
1 & 0 \\
0 & 1 \\
0 & 0
\end{array}\right] \quad \text { e } \quad \boldsymbol{\epsilon}_{t}=\left[\begin{array}{l}
1 \\
0 \\
1
\end{array}\right] \gamma_{t} .
$$

A partir do momento $t$ em diante a variável latente passa a ter uma dimensão a mais e nos períodos posteriores ao momento de intervenção a matriz de evolução $\boldsymbol{G}_{t}$ e matriz de variância $\boldsymbol{W}_{t}$ passa a ter uma coluna e linha de zeros adicional. Assim, a cada nova observação avaliada, a posteriori para $\gamma_{t}$ é revisada, aprendendo sobre a mudança ocorrida no tempo $t$ (West e Harrison, 1997, Cap. 11, Sec. 2). 


\section{Apêndice C}

\section{Modelo linear dinâmico generalizado}

Nesta abordagem, a suposição de que as observações sejam normalmente distribuídas é relaxada. De forma bem mais abrangente, assume-se, simplesmente, que a distribuição das observações seja membro da familia exponencial de Nelder e Wedderburn (1972), isto é:

$$
p\left(y_{t} \mid \eta_{t}\right)=\exp \left\{\phi_{t}^{-1}\left[y_{t} \eta_{t}-b\left(\eta_{t}\right)\right]+c\left(y_{t}, \phi_{t}\right)\right\}
$$

onde $\eta_{t}$ é o parâmetro natural e $\phi_{t}=V_{t}^{-1}$ é a precisão da distribuição. Neste caso, $\eta_{t}$ é o parâmetro que tem uma relação linear com o vetor de estados $\theta_{t}$, mas a equação de evolução permanece a mesma em relação ao modelo normal, exceto pela distribuição dos erros. A distribuição normal faz parte desta família, portanto o modelo normal é um caso particular do modelo dinâmico generalizado.

O modelo dinâmico generalizado é especificado por:

- Função Média: $\mu_{t}=E\left(Y_{t} \mid \eta_{t}\right)=b^{\prime}\left(\eta_{t}\right)=\frac{\partial b\left(\eta_{t}\right)}{\partial \eta_{t}}$;

- Função de ligação: $\eta_{t}=g\left(\mu_{t}\right)=F_{t}^{\prime} \theta_{t}$;

- Equação do sistema: $\theta_{t}=G_{t} \theta_{t-1}+\omega_{t}$;

- Erro do sistema: $\omega_{t} \sim\left(0, W_{t}\right)$ (observa-se que somente os momentos estão definidos, mas não a distribuição de $\left.\omega_{t}\right)$;

- Informação a priori: $\theta_{1} \sim\left(a_{1}, R_{1}\right)$

(da mesma forma, não é imposta uma distribuição específica para $\theta_{1}$ ).

Observa-se que, no caso da distribuição normal, o parâmetro natural $\eta_{t}$ é a própria média $\mu_{t}$, logo $g\left(\mu_{t}\right)=\mu_{t}$.

A sequência de procedimentos utilizada na estimação do modelo é sumarizada a seguir.

- Posteriori em $t-1$ : $\left(\theta_{t-1} \mid D_{t-1}\right) \sim\left(m_{t-1}, C_{t-1}\right)$, e

- Priori em $t:\left(\theta_{t} \mid D_{t-1}\right) \sim\left(a_{t}, R_{t}\right)$

onde $a_{t}=G_{t} m_{t-1}$ e $R_{t}=G_{t} C_{t-1} G_{t}^{\prime}+W_{t}$.

- A priori para $\eta_{t}$ é dada por: $\left(\eta_{t} \mid D_{t-1}\right) \sim\left(f_{t}, q_{t}\right)$.

No caso em que a priori de $\eta_{t}$ é conjugada, tem-se que priori e posteriori pertencem na mesma família e portanto $\left(\eta_{t} \mid D_{t}\right) \sim\left(f_{t}^{*}, q_{t}^{*}\right)$.

- A distribuição conjunta de $\left(\eta_{t}, \theta_{t} \mid D_{t-1}\right)$, considerando apenas os momentos, é dada por:

$$
\left(\eta_{t}, \theta_{t} \mid D_{t-1}\right) \sim\left[\left(\begin{array}{c}
f_{t} \\
a_{t}
\end{array}\right),\left(\begin{array}{cc}
q_{t} & F_{t}^{\prime} R_{t} \\
R_{t} F_{t} & R_{t}
\end{array}\right)\right]
$$

onde $f_{t}=F_{t}^{\prime} a_{t}$ e $q_{t}=F_{t}^{\prime} R_{t} F_{t}$. 
- Uma vez conhecidos $f_{t}$ e $q_{t}$, os parâmetros da priori conjugada de $\mu_{t}$ são obtidos pela relação:

$$
f_{t}=E\left[g\left(\mu_{t}\right) \mid D_{t-1}\right] \quad \text { e } \quad q_{t}=V\left[g\left(\mu_{t}\right) \mid D_{t-1}\right]
$$

- Os momentos associados a $\left(\theta_{t} \mid \eta_{t}, D_{t-1}\right)$ são estimados via Linear Bayes (vide West e Harrison, 1997; cap. 4.9):

$$
\begin{aligned}
& \widehat{E}\left(\theta_{t} \mid \eta_{t}, D_{t-1}\right)=a_{t}+R_{t} F_{t}\left(\eta_{t}-f_{t}\right) / q_{t} \\
& \widehat{V}\left(\theta_{t} \mid \eta_{t}, D_{t-1}\right)=R_{t}-R_{t} F_{t} F_{t}^{\prime} R_{t} / q_{t} .
\end{aligned}
$$

- Após atualizada a posteriori $\left(\mu_{t} \mid D_{t}\right)$ e obtidos $E\left[\mu_{t} \mid D_{t}\right]$ e $V\left[\mu_{t} \mid D_{t}\right]$, é mantida a relação:

$$
f_{t}^{*}=E\left[g\left(\mu_{t}\right) \mid D_{t}\right] \quad \text { e } \quad q_{t}^{*}=V\left[g\left(\mu_{t}\right) \mid D_{t}\right],
$$

$\operatorname{com}\left(\eta_{t} \mid D_{t}\right) \sim\left(f_{t}^{*}, q_{t}^{*}\right)$.

- A posteriori em $t,\left(\theta_{t} \mid D_{t}\right)$, segue de:

$$
p\left(\theta_{t} \mid D_{t}\right)=\int p\left(\theta_{t} \mid \eta_{t}, D_{t-1}\right) p\left(\eta_{t} \mid D_{t}\right) d \eta_{t}
$$

onde $p\left(\theta_{t} \mid \eta_{t}, D_{t-1}\right)$ não é conhecida, mas os momentos de $\left(\theta_{t} \mid D_{t}\right)$ podem ser estimados por:

$$
\begin{aligned}
m_{t} & =E\left(\theta_{t} \mid D_{t}\right) \\
& =E\left[\widehat{E}\left(\theta_{t} \mid \eta_{t}, D_{t-1}\right) \mid D_{t}\right] \\
& =a_{t}+R_{t} F_{t}\left(f_{t}^{*}-f_{t}\right) / q_{t} \\
\mathrm{e} & \\
C_{t} & =V\left(\theta_{t} \mid D_{t}\right) \\
& =V\left[\widehat{E}\left(\theta_{t} \mid \eta_{t}, D_{t-1}\right) \mid D_{t}\right]+E\left[\widehat{V}\left(\theta_{t} \mid \eta_{t}, D_{t-1}\right) \mid D_{t}\right] \\
& =R_{t}-R_{t} F_{t} F_{t}^{\prime} R_{t}\left(1-q_{t}^{*} / q_{t}\right) / q_{t} .
\end{aligned}
$$




\section{Apêndice D}

\section{Principais programações em $R$}

Os códigos para as funções de filtro de partículas foram construídos com auxílio dos exemplos de Lopes e Tsay (2011) disponíveis em <http://hedibert.org/r-code-to-our-journal-of-forecastingreview-paper $/>$.

\section{D.1 Simulação dos dados BIZU dinâmico}

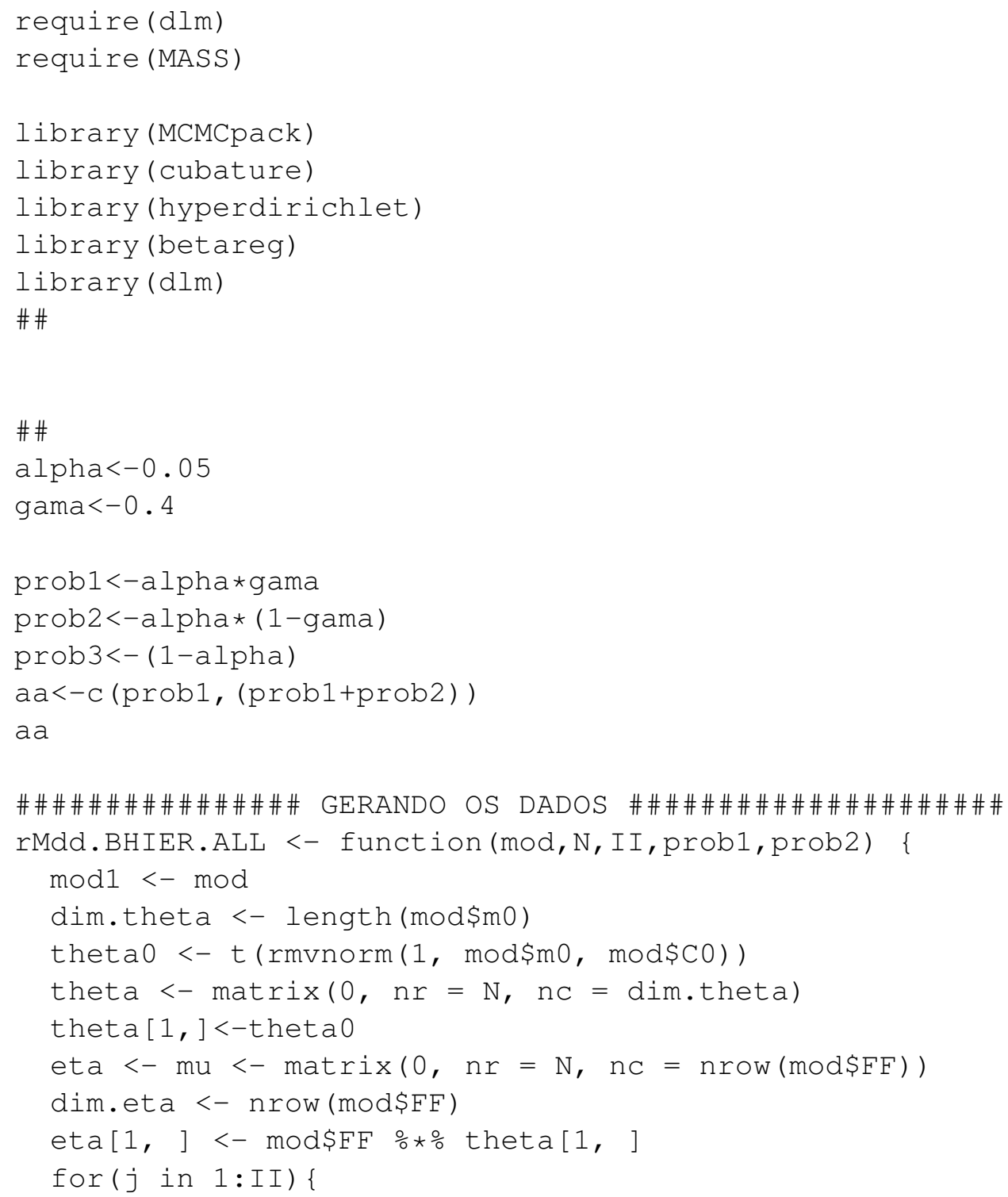




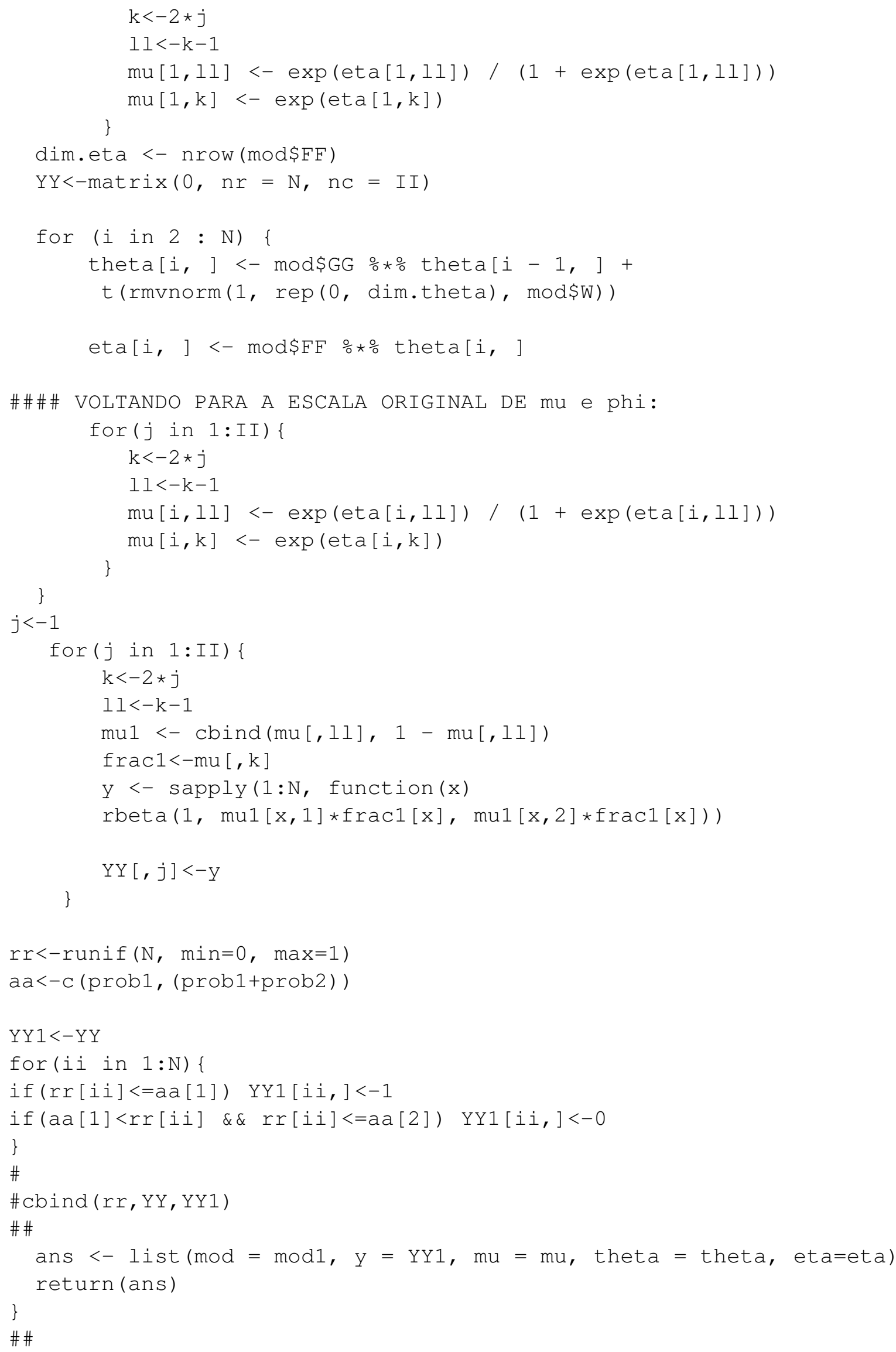




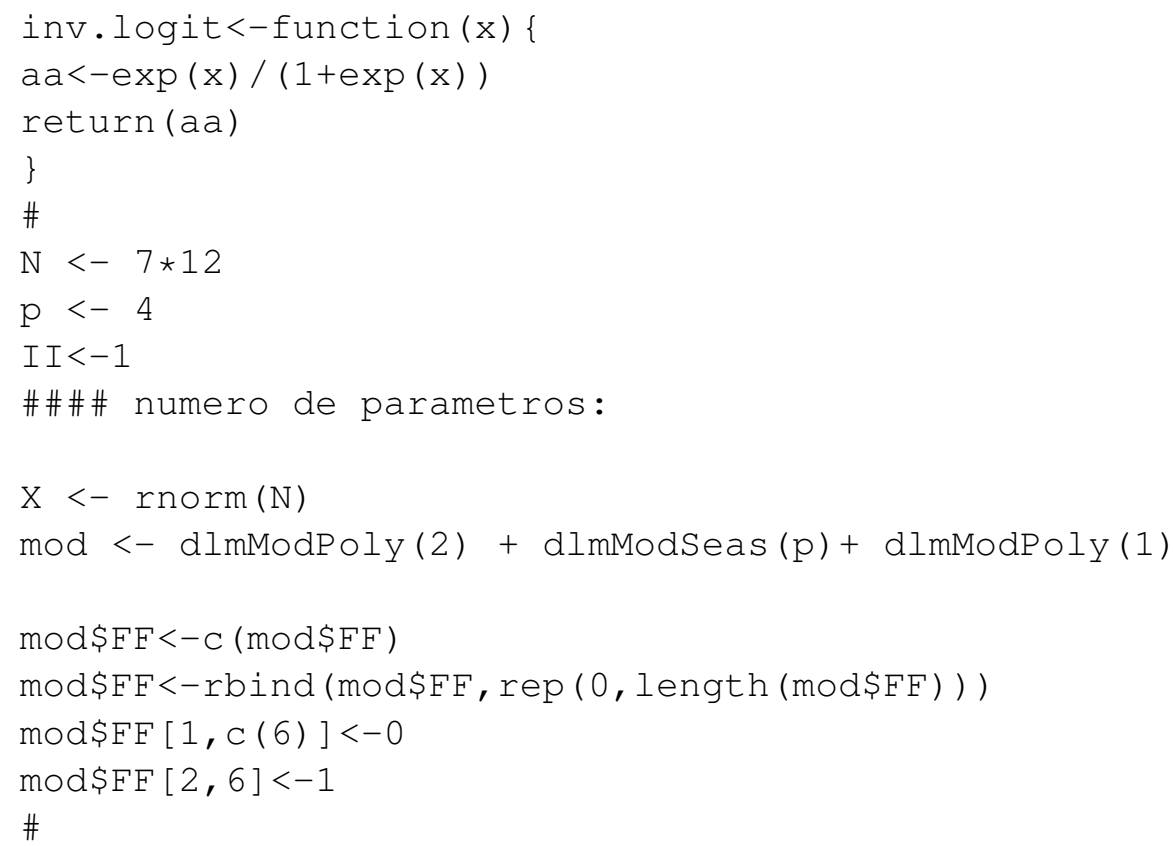

\section{D.2 Simulação dos dados Tobit dinâmico}

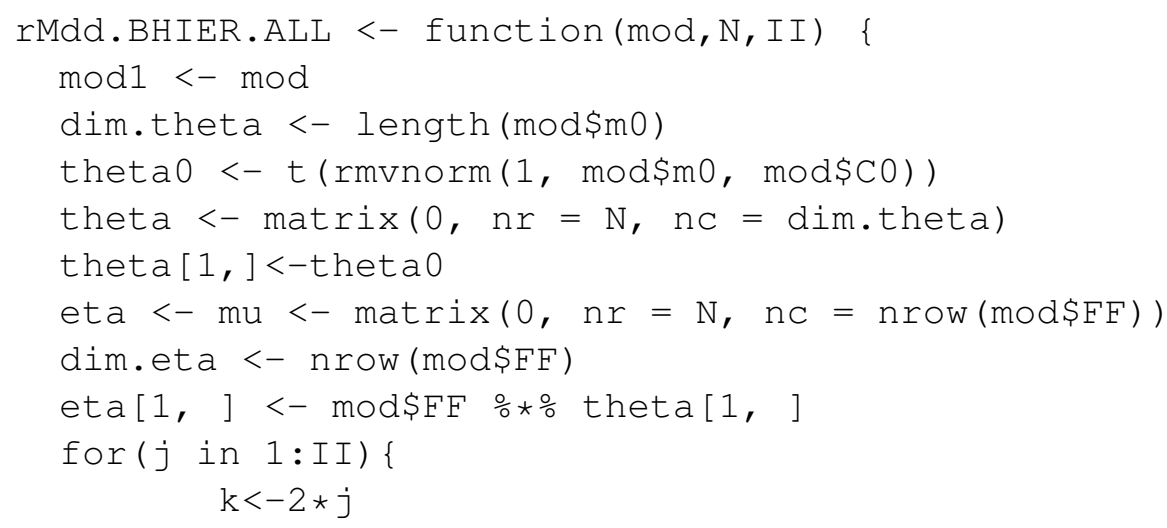




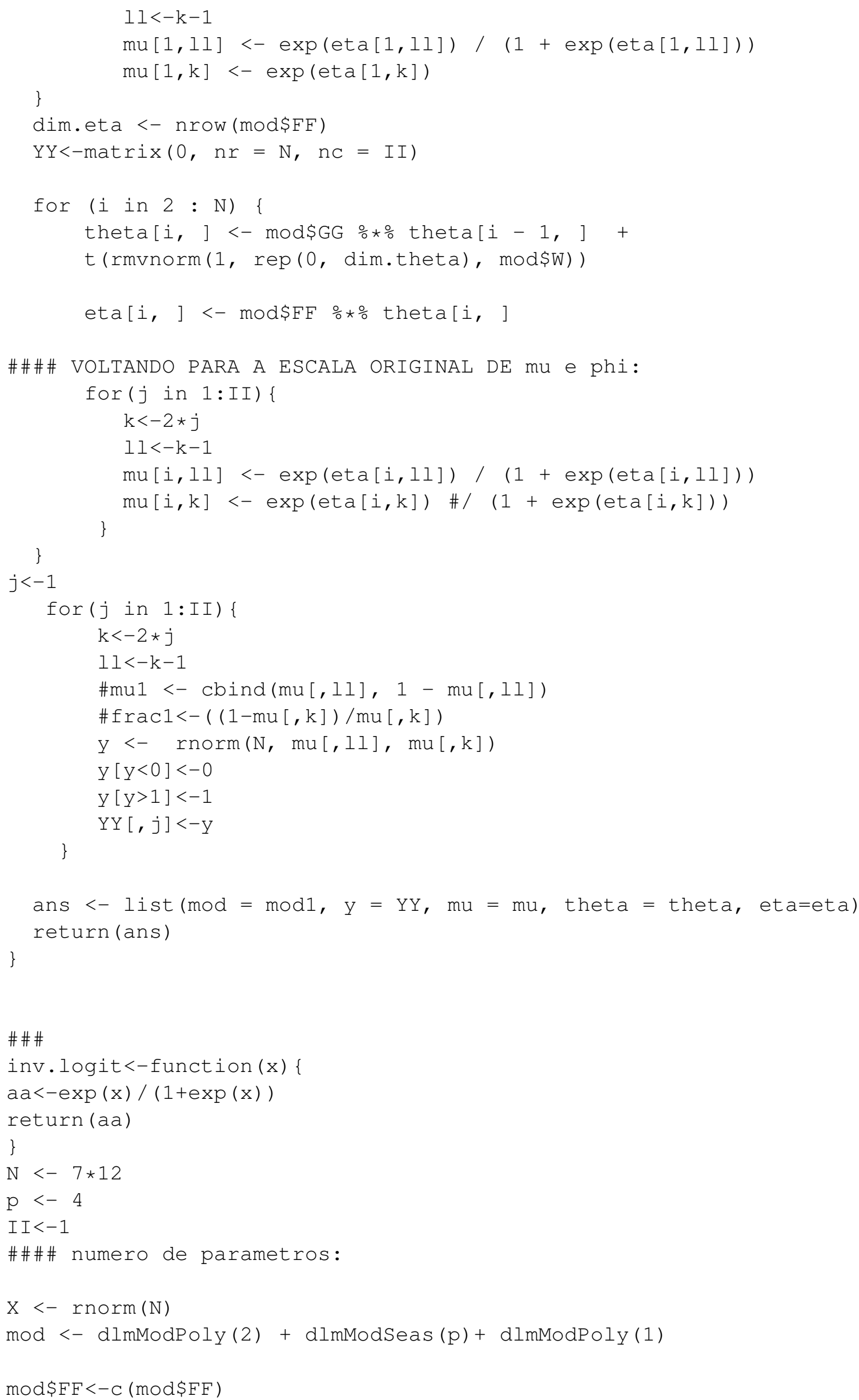




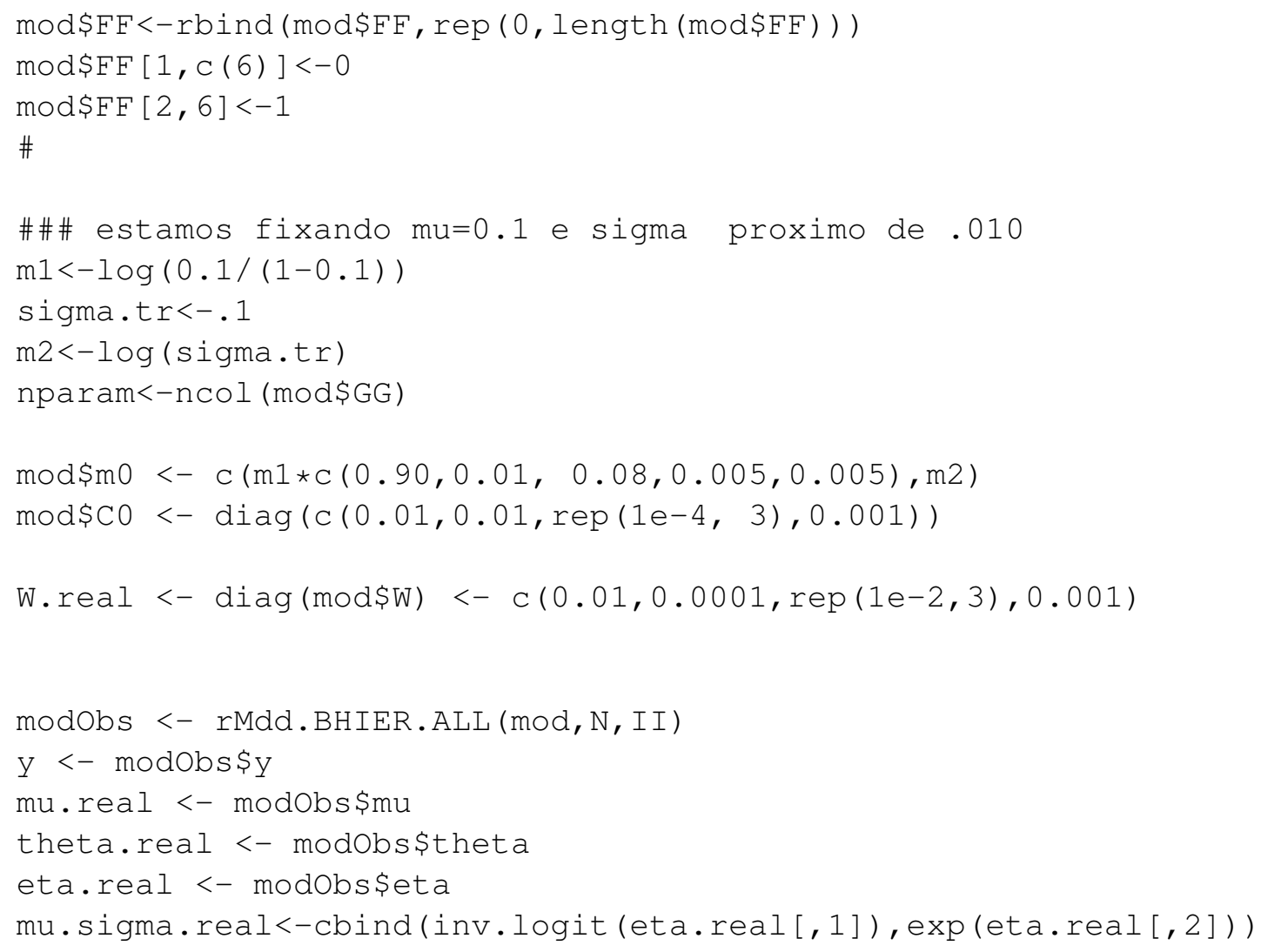

\section{D.3 Filtro PL para o modelo dinâmico BIZU}

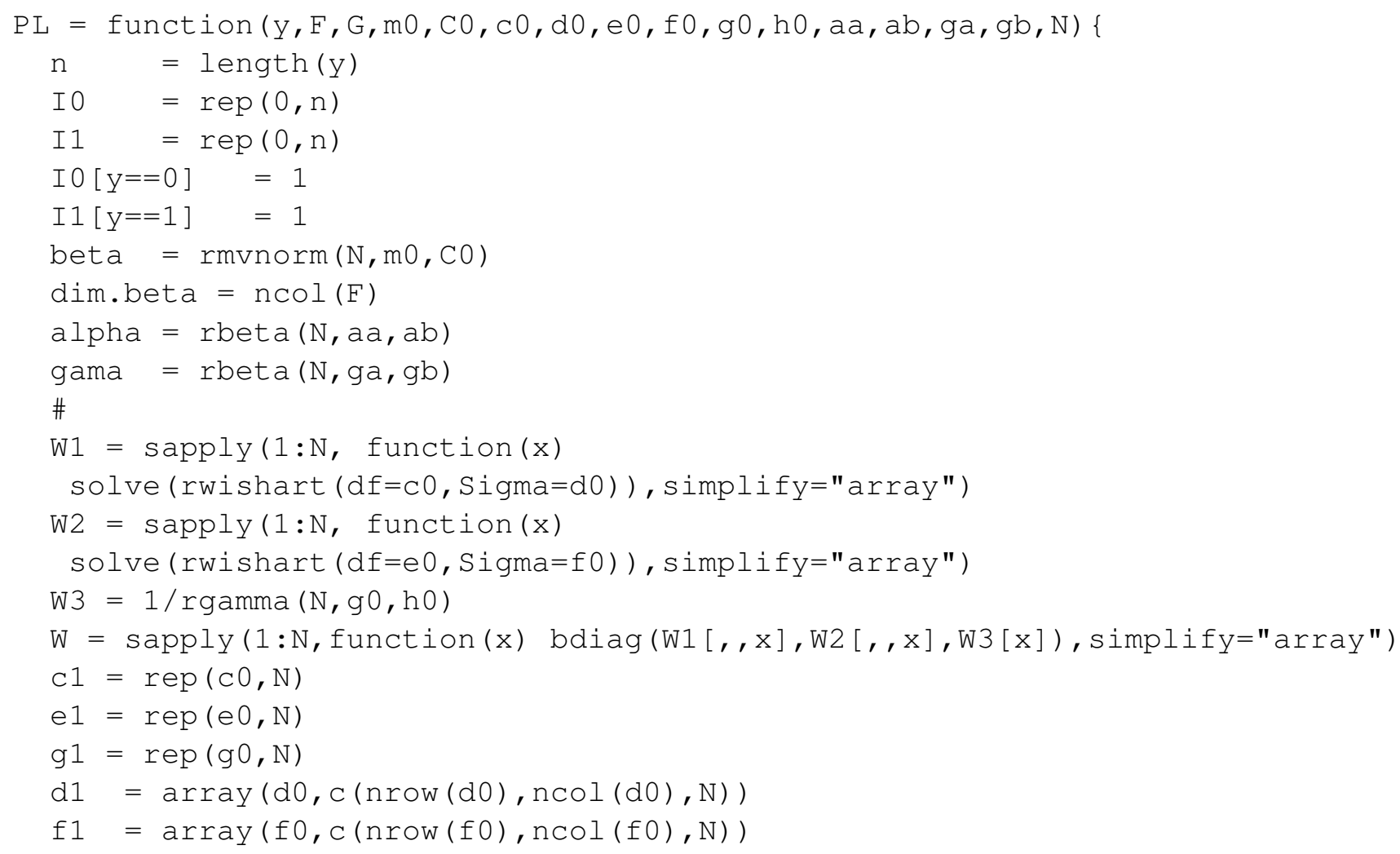




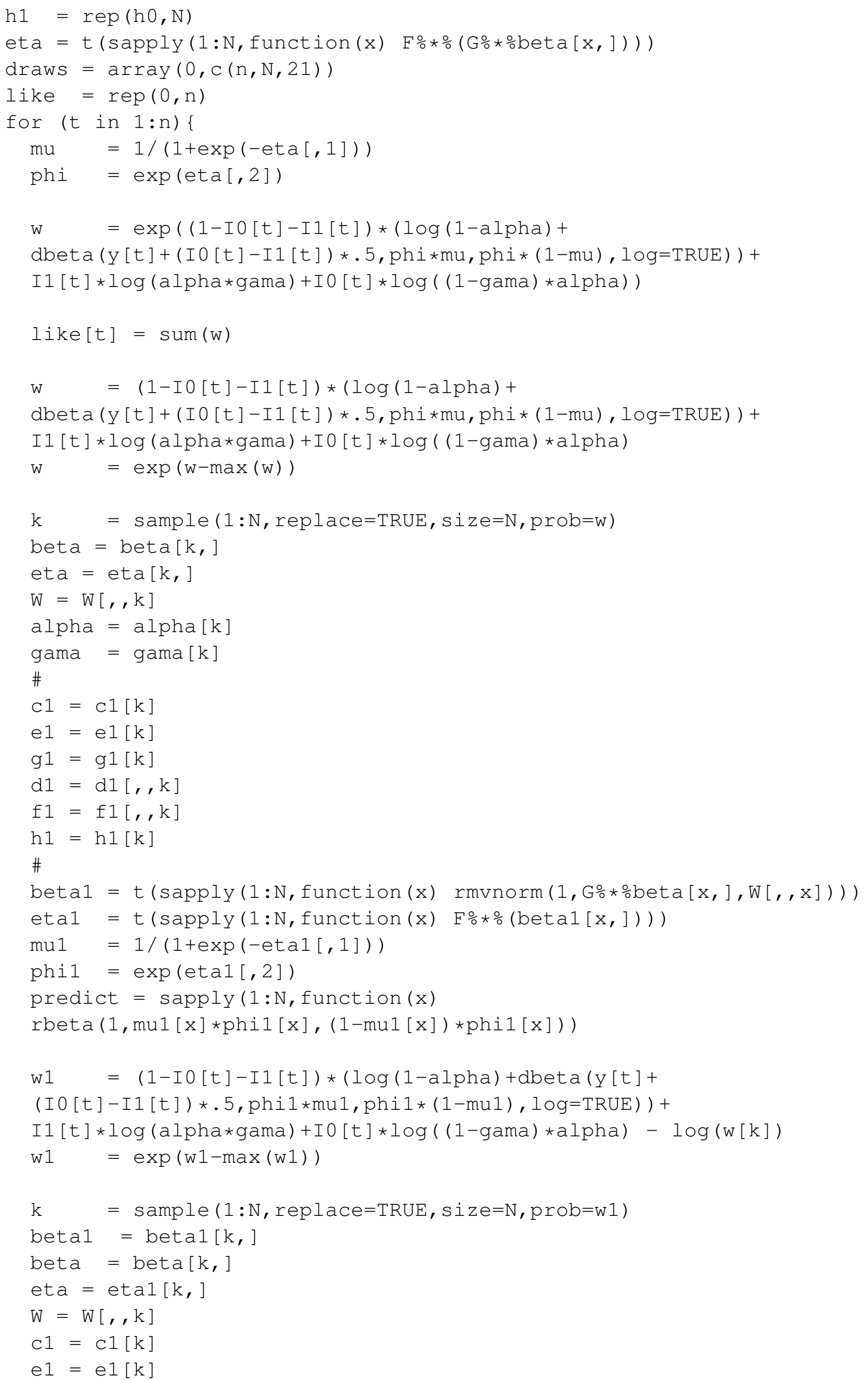




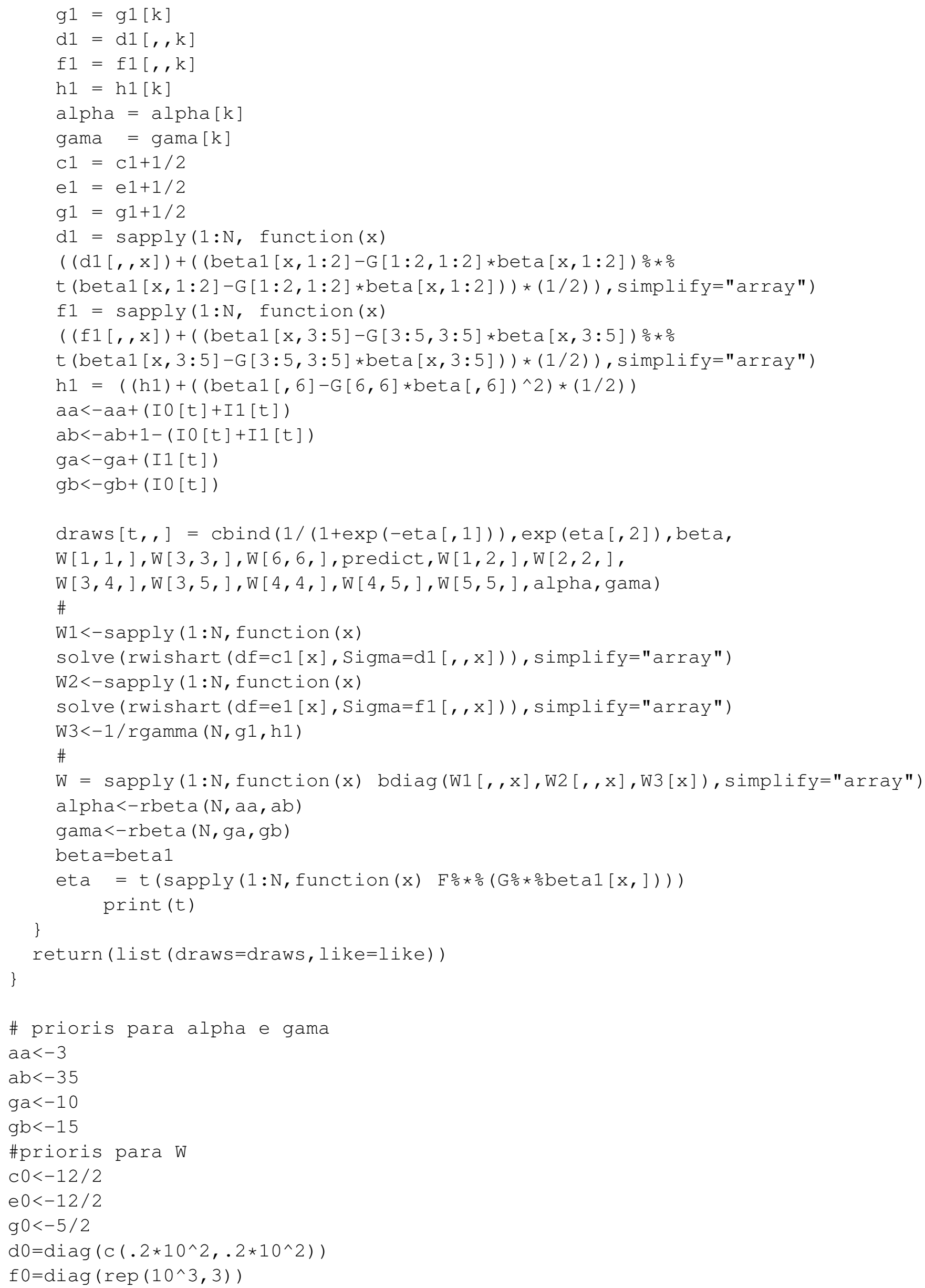




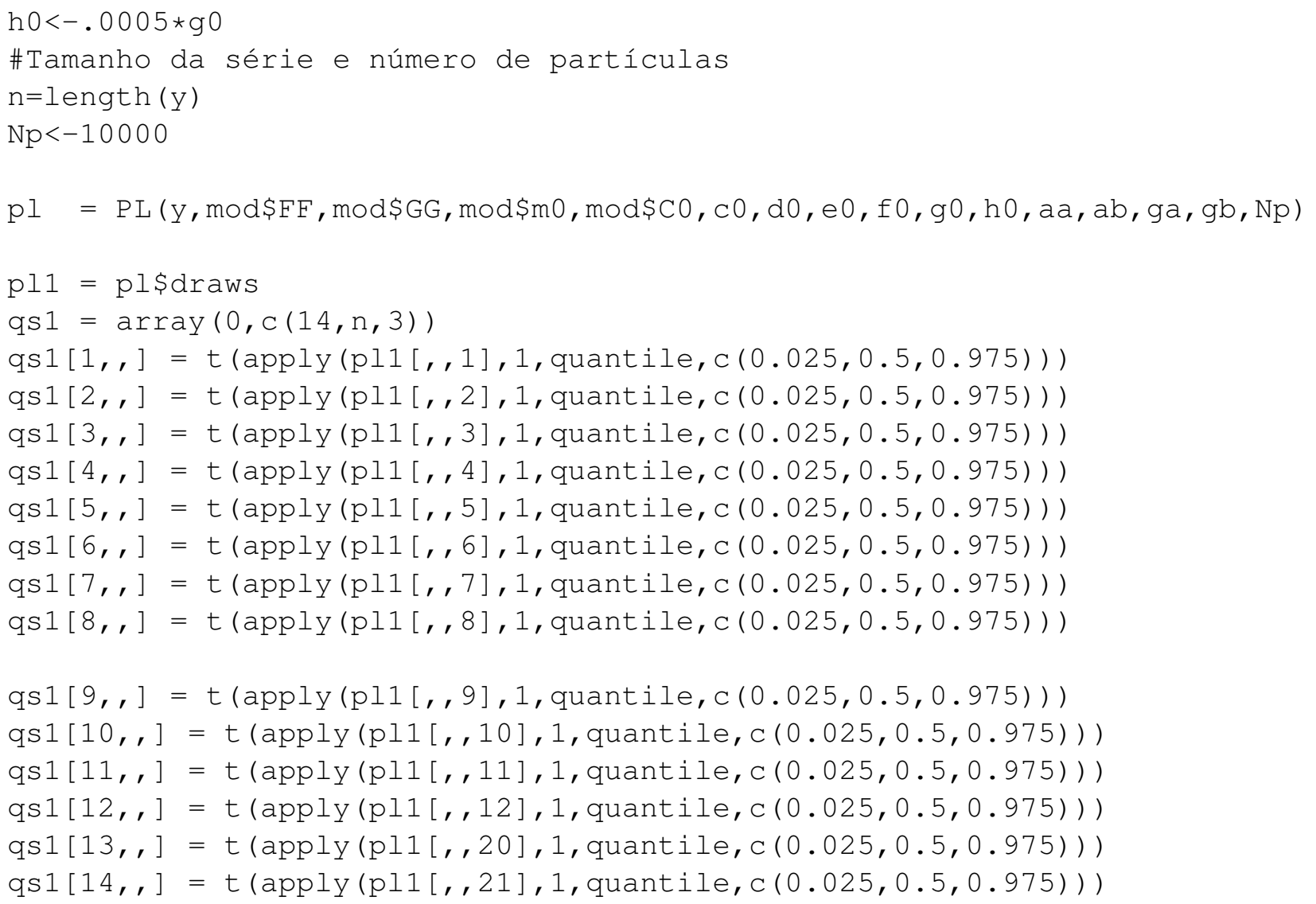

\section{D.4 Filtro PL para o modelo dinâmico Tobit}

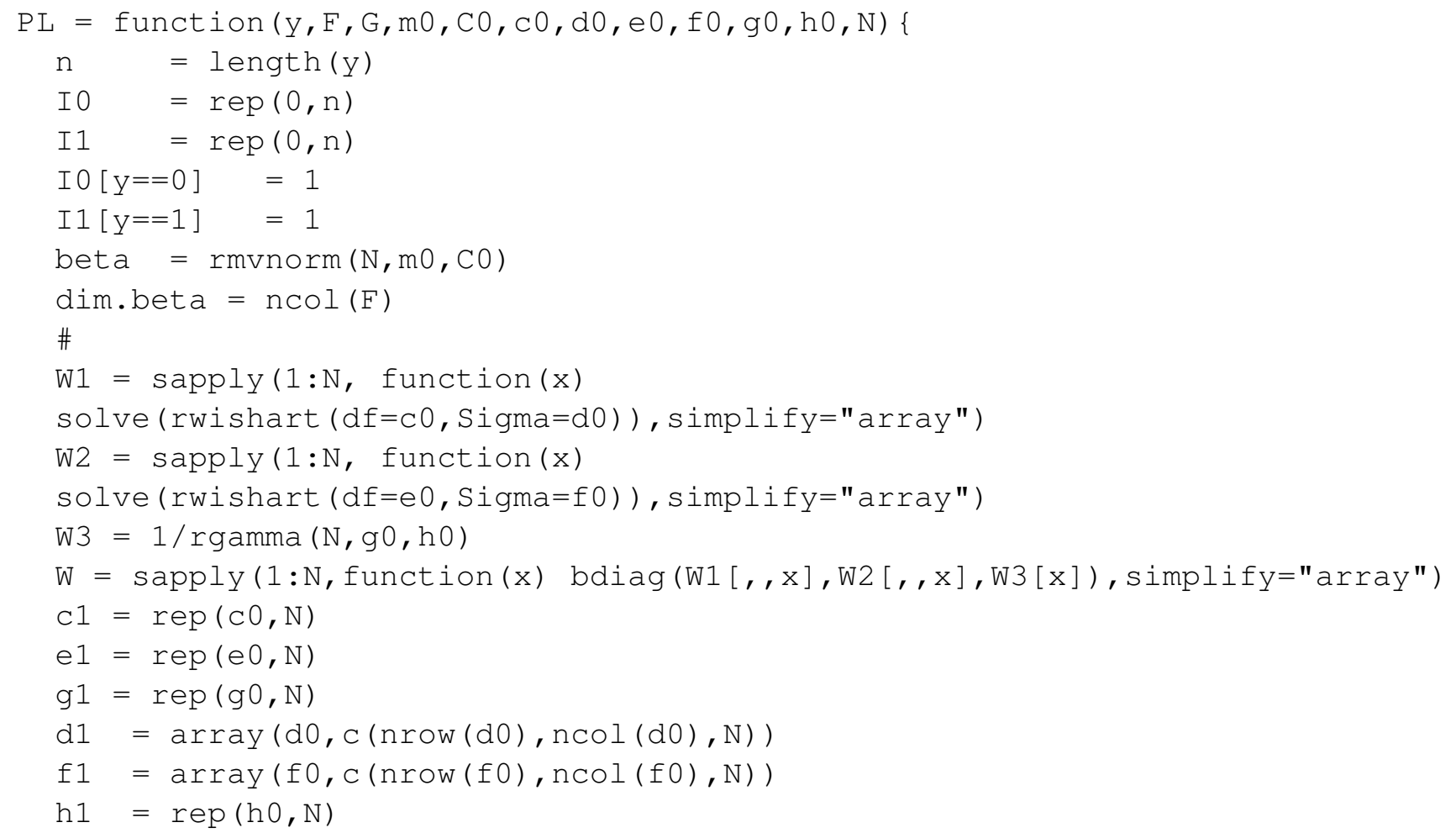




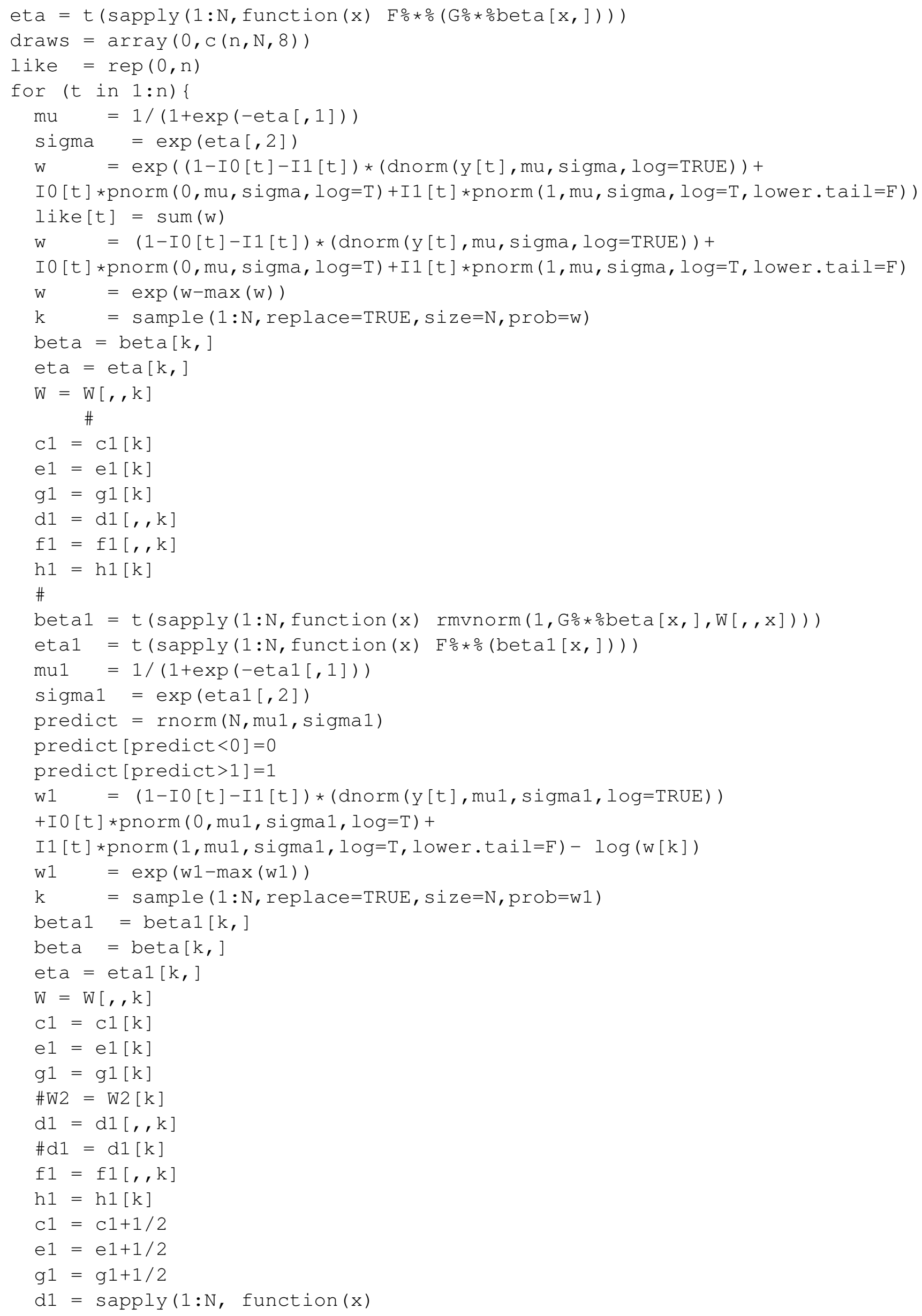




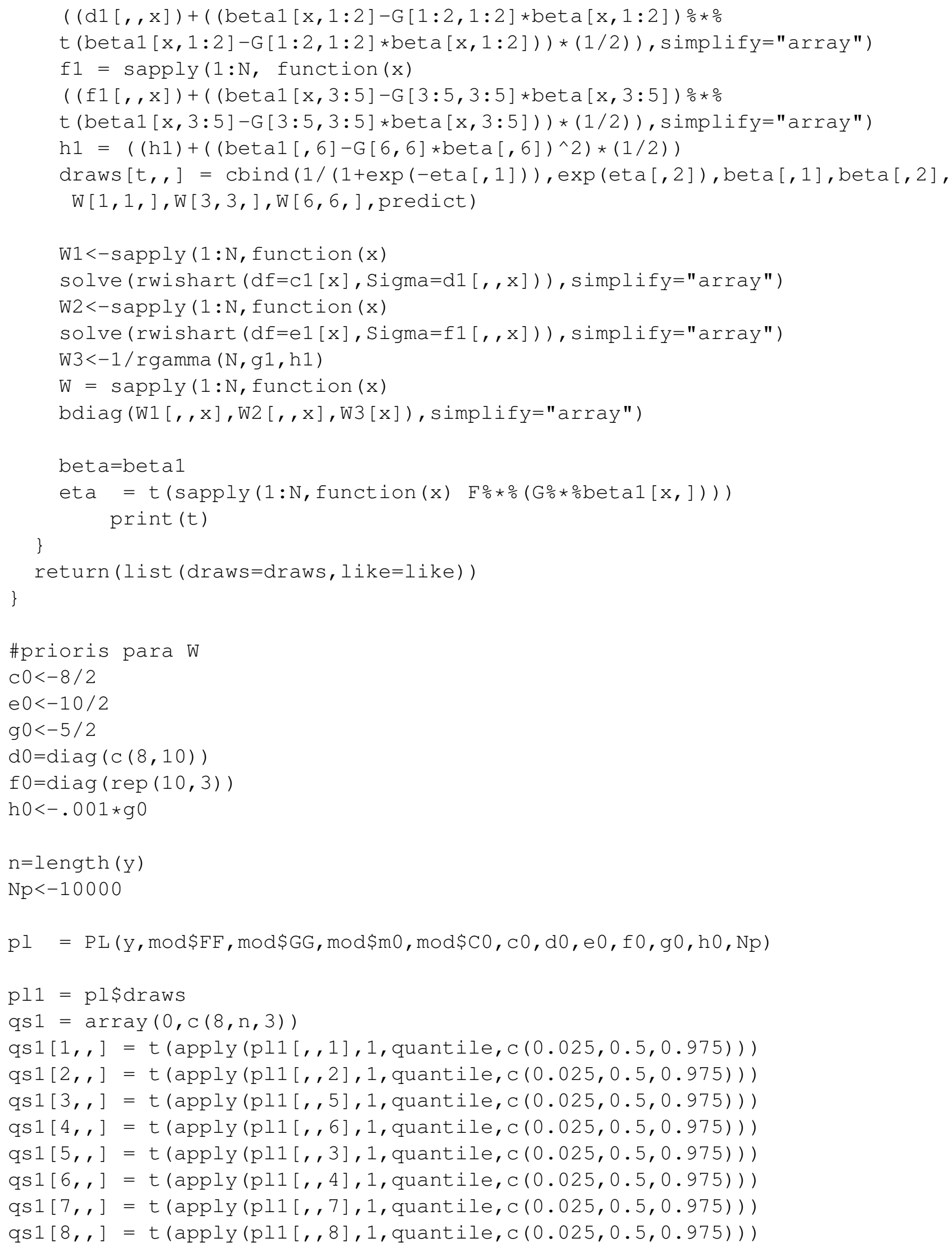




\section{Referências Bibliográficas}

Aitchison(1982) J. Aitchison. The statistical analysis of compositional data. Jornal of Royal Statistic Societ. Series B (Methodological), 44(2):139-177. Citado na pág. 1

Albi et al.(2009) M. Albi, H. S. Migon e C. Q. da Silva. Bayesian inference for beta distribution. Relatório técnico, Departamento de Estatística - Universidade de Brasília. Citado na pág. 1

Bayes et al.(2012) C. Bayes, J. Bazan e C. García. A new robus regression model for proportions. Bayesian Analysis, 7:841-866. Citado na pág. 1

Branscum et al.(2007) A. J. Branscum, W. O. Johnson e M. C. Thurmon. Bayesian beta regression: Applications to household expenditure data and genetic distance between foot-and-mouth disease viruses. Aust. N. Z. J. Stat., 49(3):287-301. Citado na pág. 1

Carter e Kohn(1994) C. K. Carter e R. Kohn. On gibbs sampling for state-space models. Biometrika, 81:541-553. Citado na pág. 64

Carvalho et al.(2010) C. M. Carvalho, M. S. Johannes, H. F. Lopes e N. G. Polson. Particle learning and smoothing. Statistical Science, 25:88-106. Citado na pág. 78

Chai e Baikey(2008) H. s. Chai e K. R. Baikey. Use of log-skew-normal distribution in analysis of continuous data with a discrete component at zero. Statist. Med., 27:3643-3655. Citado na pág. 143

Chib(1992) Chib. Bayes inference in the tobit censored regression model. Journal of Econometrics, 51:79-99. Citado na pág. 1

Cragg(1971) J. Cragg. Some statistical models for limited dependent variables with application to the demand for durable goods. Econometrica, 39:829-844. Citado na pág. 1, 3, 13, 24, 26

Csiszár(1967) I. Csiszár. Information-type measures of diference of probability distributions and indirect observations. Studia Scientiarum Mathematicarum Hungarica, 2:299-318. Citado na pág. 32

da-Silva e Migon(2012) C. Q. da-Silva e H. S. Migon. Hierarchical dynamic beta model. Relatório técnico, 253. Departamento de Métodos Estatísticos - Universidade Federal do Rio de Janeiro. Citado na pág. 2, 19, 55, 58, 65

da-Silva et al.(2011) C. Q. da-Silva, H. S. Migon e L. T. Correia. Dynamic bayesian beta model. Jornal of Computational Statistics and Data Analysis, 55:2074-2089. Citado na pág. 2, 3, 27, 55, 59, 68

Dagenais(1982) M. G. Dagenais. The tobit model with serial correlation. Economics Letters, 10: 263-267. Citado na pág. 2

De Jong(1991) P. De Jong. The diffuse kalman filter. Annals of Statistics, 19:1073-83. Citado na pág. 64 
Dey et al.(1997) D. K. Dey, M. H Chen e H. Chang. Bayesian approach for the nonlinear random effects models. Biometrics, 53:1239-52. Citado na pág. 30

Douc e Cappe(2006) A. Douc e O. Cappe. Comparison of resampling scheme of particle filter. Proceeding of the 4 th International Symposium on Image and Signal Processing and Analysis, páginas 64-69. Citado na pág. 71

Doucet et al.(2004) A. Doucet, M. West e S. J. Godsill. Monte carlo smoothing for non-linear time series. Jornal of Americam Statistical Association, 140:107-113. Citado na pág. 77, 79

Ferrari e Cribari-Neto(2004) S. L. P. Ferrari e F. Cribari-Neto. Beta regression for modeling rates and proportions. Jornal of Applied Statistics, 10:1-18. Citado na pág. 1, 6

Früwirth-Schmatter(1994) S. Früwirth-Schmatter. Data augmentation and dynamic linear models. Journal of the Royal Statistical Society: Series B, 55:629-642. Citado na pág. 64

Gamerman(1991) D. Gamerman. Dynamic bayesian models for survival data. Applied Statistics, 40:63-79. Citado na pág. 2

Gamerman(1998) D. Gamerman. Markov chain monte carlo for dynamic generalized linear mixed models. Biometrika, 85:215-27. Citado na pág. 2

Gamerman e West(1987) D. Gamerman e M. West. A time series application of dynamic survival models in unemployment studies. The Statistician, 36:174-269. Citado na pág. 2

Garay et al.(2012) A. M. Garay, V. H Lachos e H. Bolfarine. Bayesian zero-inflated negative binomial regression models: estimation and case influence diagnostics. Submetido para publicação, 2012. Citado na pág. 33

Gelfand e Smith(1990) A. E. Gelfand e A. F. M. Smith. Sampling based approaches to calculating marginal densities. JASA, 85:410. Citado na pág. 2

Geman e Geman(1984) S. Geman e D. J. Geman. Stochastic relaxation, gibbs distributions and the bayesian restoration of images. IEEE Transactions on Pattern Analysis and Machine Intelligence, 6:721-741. Citado na pág. 2

Gómez et al.(2009) H. W. Gómez, D. Elal-Olivero, H. S. Salinas e H. Bolfarine. Bimodal extension based on the skew-normal distribution with application to pollen data. Envirometrics, 22:50-62. Citado na pág. 144

Godolphin e Triantafyllopoulos(2006) E. J. Godolphin e K. Triantafyllopoulos. Decomposition of time series models in state-space form. Computational Statistics and Data Analysis, 50:22322246. Citado na pág. 2

Gordon et al.(1993) N. J. Gordon, D. J. Salmond e A. F. M. Smith. Novel approach to nonlinear/non-gaussian bayesian state estimation. IEEE Proceedings F on Radar and Signal Processing, 140:107-113. Citado na pág. 2, 68, 72

Gupta e Gupta(2008) D. Gupta e R. C. Gupta. Analyzing skewed data by power normal model. Test, 17:197-210. Citado na pág. 2, 3, 16

Hastings(1970) W. K. Hastings. Monte carlo sampling methods using markov chains and their applications. Biometrika, 57:97-109. Citado na pág. 28

Johnson et al.(1995) N. Johnson, S. Kotz e N. Balakrishnan. Continuous Univariate Distributions. John Wiley and Sons, 2nd ed. edição. Citado na pág. 1 
Kieschnick e McCullough(2003) R. Kieschnick e B. D. McCullough. Regression analysis of variates observed on $(0,1)$ : percentages, proportions, and fractions. Statistical Modelling, 3:193213. Citado na pág. 1

Kumar(2004) S. Kumar. Mixed type distributions. website, 2004. Disponível em: $<$ http://homepage.stat.uiowa.edu/nshyamal/22S175/DI.pdf $>$. Citado na pág. 5

Lee(1997) L. Lee. Estimation of dynamic and arch tobit models. Working paper, Department of Economics, 1997. Citado na pág. 2

Lehmann(1953) E. L. Lehmann. A graphical estimation of mixed weibull parameter in life testing electron tubes. Technometrics, 1:389-407. Citado na pág. 2, 16

Lindsey e Lambert(1995) J. K. Lindsey e P. Lambert. Dynamic generalized linear models and repeated measurements. Journal of Statistical Planning and Inference, 47:129-139. Citado na pág. 2

Liu e West(2001) J. Liu e M. West. Combined parameter and state estimation in simulation-based filtering. Citado na pág. 76

Lopes(2012) H. F. Lopes. Simulation-based approaches to modern bayesian econometrics. website, 2012. Disponível em: <http://http://hedibert.org/simulation-based-approaches-to-modernbayesian-econometrics $>$. Citado na pág. 65

Lopes e Carvalho(2013) H. F. Lopes e C. M. Carvalho. Online bayesian learning in dynamic models: An illustrative introduction to particle methos. Em Bayesian Theory and Applications. Wet, M., Damien, P., Dellaportas,P., Polson, N. G. and Stephens, D. A. (Eds). Clarendon: Oxford University Press. Citado na pág. 79

Lopes e Tsay(2011) H. F. Lopes e Tsay. Particle filters and bayesian inference in financial econometrics. Journal of Forecasting, 30:168-209. Citado na pág. 151

Lopes et al.(2011) H. F. Lopes, C. M. Carvalho, M. S. Johannes e N. G. Polson. Particle learning for sequential bayesian computation. Bayesian Statistics, 9:317-360. Citado na pág. 78, 79

Martinez et al.(2012) G. Martinez, H. Bolfarine e H. W. Gómez. Asymmetric regression models with limited responses with an application to antibody response to vaccine. Biometrical Journal, página Aceito para publicação. Citado na pág. 144

McCormick et al.(2012) T.H. McCormick, A.E. Raftery e Burd R.S. Madigan, D. Dynamic logistic regression and dynamic model averaging for binary classification. Biometrics, 68:23-30. Citado na pág. 59, 144

McDowell(2003) A. McDowell. From the help desk: hurdle models. The Stata Jornal, 3:172-184. Citado na pág. 1

Metropolis et al.(1953) N. Metropolis, A. W. Rosenbluth, M. N. Rosenbluth, A. H. Teller e E. Teller. Equations of state calculations by fast computing machines. J. Chem. Phys, 21: 1087-92. Citado na pág. 28

Migon(1984) H. S. Migon. An approach to non-linear Bayesian forecasting problems with applications. Tese de Doutorado, University of Warwick, Department of Statistics. Citado na pág. 2

Migon e Harrison(1985) H. S. Migon e P. J. Harrison. An application of non-linear bayesian forecasting to television advertising. Em Bayesian Statistics 2. J.M. Bernardo, M.H. DeGroot, D.V. Lindley, and A.F.M. Smith (Eds.). North-Holland, Amsterdam, and Valencia University Press. Citado na pág. 2 
Moulton e Halsey(1995) L. Moulton e N. A. Halsey. A mixture model with detection limits for regression analyses of antibody response to vaccine. Biometrics, 51:1570-78. Citado na pág. 1, 3, $11,22,55$

Mroz(1987) T. Mroz. The sensitivity of an empirical model of married women's hours of work to economical and statistical assumptions. Econometrica, 55:765-799. Citado na pág. 1

Nascimento(2014) I. F. Nascimento. Modelos dinâmicos com estimação via filtro de partículas. Dissertação de Mestrado, Universidade de Brasília, Departamento de Estatística, 2014. Citado na pág. 79

Nelder e Wedderburn(1972) J. A. Nelder e R. W. M. Wedderburn. Generalized linear models. Journal of the Royal Statistical Society, 135:370-384. Citado na pág. 149

Ospina(2008) R. Ospina. Modelos de regressão beta inflacionados. Tese de Doutorado, Universidade de São Paulo, Brasil. Citado na pág. 1, 2, 3, 6, 7, 19, 20, 31, 33, 38, 55

Ospina e Ferrari(2010) R. Ospina e S. L. P. Ferrari. Inflated beta distributions. Statistical Papers, 51:111-126. Citado na pág. 2

Ospina e Ferrari(2012) R. Ospina e S. L. P. Ferrari. A general class of zero-or-one inflated beta regression models. Computational Statistics and Data Analysis, 56:1609-23. Citado na pág. 1

Paolino et al.(2003) C. Paolino, M. Amaral e B. Murteira. Estatística Bayesiana. Fundação Calouste Gulbenkian. Citado na pág. 2, 30

Paolino(2001) P. Paolino. Maximum likelihood estimation of models with beta-distributed dependent variables. Political Analysis, 9:325-346. Citado na pág. 1

Peng e Dey(1995) F. Peng e D. K. Dey. Bayesian analysis of outlier problems using divergence measures. The Canadian Journal of Statistics, 27:859-870. Citado na pág. 2, 31, 33

Pereira et al.(2012) G.H.A. Pereira, D.A. Botter e M.C. Sandoval. The truncated inflated beta distribution. Statist. Med., 13:125-151. Citado na pág. 144

Petris et al.(2009) G. Petris, S. Petrone e P. Campagnoli. Dynamic Linear Models with R. Springer. Citado na pág. 58

Pewsey et al.(2012) A. Pewsey, H. W. Gómez e H. Bolfarine. Likelihood-based inference for distributions of fractional order statistics. Test, 21:775-789. Citado na pág. 2, 3, 16

Pitt e Shephard(1999) Pitt e Shephard. Filtering via simulation: auxiliary partical filters. Journal of the American Statistical Association, 94:590-599. Citado na pág. 2, 59, 73, 74, 75, 78

Rubin(1992) D. B. Rubin. The calculation of posterior distributions by data augmentation. Jornal of the American Association. Citado na pág. 74

Rudin(1976) W. Rudin. Principals of Mathematical Analysis. McGraw-Hill, 3rd edição. Citado na pág. 21

Smith e Gelfand(1992) F. M. Smith e A. E. Gelfand. Bayesian statistic without tears: A sampling-resampling perspective. The American Statisticia, 46:84-88. Citado na pág. 73

Souza(2014) P. O. Souza. Modelos dinâmicos para a distribuição poisson generalizada. Dissertação de Mestrado, Universidade de Brasília, Departamento de Estatística, 2014. Citado na pág. 59

Spiegelhalter et al.(2002) D. Spiegelhalter, N. Best, B. Carlin e A. Van der Linde. Bayesian measures of model complexity and fit. Journal of the Royal Statistical Society: Series B, 64: 583-639. Citado na pág. 29 
Storvik(2002) G. Storvik. Particle filters for state-space models with presence of unknown static parameters. IEEE Transactions on Signal Processing, 50:281-298. Citado na pág. 77, 78

Tanner e Wong(1987) M. A. Tanner e W. H. Wong. The calculation of posterior distributions by data augmentation. JASA, 82:528-550. Citado na pág. 2

Tobin(1958) J. Tobin. Estimation of relationships for limited dependent variables. Econometrica, 57:24-36. Citado na pág. $1,10,55$

Wei(1999) S. Wei. A bayesian approach to dynamic tobit models. Econometric Reviews, 18(4): 417-439. Citado na pág. 2, 3, 55, 63, 66, 68

Weiss(1996) R. Weiss. An approach to bayesian sensitivity analysis. Journal of the Royal Statistical Society: Series B, 58:739-750. Citado na pág. 32

Weiss e Cook(1992) R. Weiss e R. D Cook. A graphical case statistic for assessing posterior influence. Biometrika, 79:51-55. Citado na pág. 31

West(1993a) M. West. Approximating posterior distributions by mixtures. Jornal of Royal Statistical Society, 55:409-422. Citado na pág. 76

West(1993b) M. West. Mixture models, monte carlo, bayesian updating and dynamic models. Computing Science and Statistics: Proceedings of 24th Symposium on the Interface, páginas 325333. Citado na pág. 76

West e Harrison(1997) M. West e P. J. Harrison. Bayesian Forcast and Dynamic Models. Springer Verlag, 2nd edição. Citado na pág. 56, 64, 77, 96, 130, 148

West et al.(1985) M. West, P. J. Harrison e H. S. Migon. Dynamic generalized linear models and bayesian forecasting (with discution). Jornal of the American Statistical Association, 80:73-97. Citado na pág. 2

Zeger e Brookmeyer(1986) S. L. Zeger e R. Brookmeyer. Regression analysis with censored autocorrelation. JASA, 81:722-729. Citado na pág. 2 